\title{
Identification and Characterization of Importin 13 Substrates
}

\author{
Dissertation \\ for the award of the degree \\ "Doctor rerum naturalium" \\ of the Georg-August-Universität Göttingen \\ within the doctoral program Molecular Biology of Cells \\ of the Georg-August University School of Science (GAUSS)
}

submitted by

Imke Baade

from Dachau

Göttingen 2017 

Thesis Committee/ Examination Board

Prof. Dr. Ralph H. Kehlenbach (referee)

Department of Molecular Biology

Georg-August-Universität Göttingen

Prof. Dr. Heike Krebber (2nd referee)

Department of Molecular Genetics

Georg-August-Universität Göttingen

Prof. Dr. Jörg Großhans

Department of Developmental Biochemistry

Georg-August-Universität Göttingen

\section{Further Members of the Examination Board}

Prof. Dr. Michael Meinecke

European Neuroscience Institute Göttingen

\section{Dr. Nuno Raimundo}

Department of Cellular Biochemistry

Georg-August-Universität Göttingen

\section{Dr. Hans Dieter Schmitt}

Department of Neurobiology

Max Planck Institute for Biophysical Chemistry

Date of oral examination: 7th September 2017 



\section{Affidavit}

I hereby declare that I have written this PhD thesis independently and with no other aids or sources than quoted.

Imke Baade

June 2017

Göttingen 



\section{Contents}

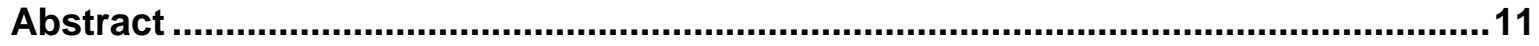

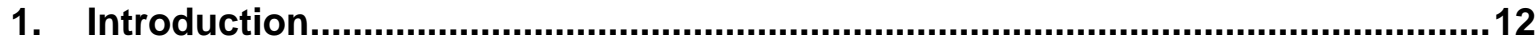

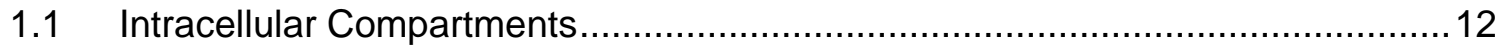

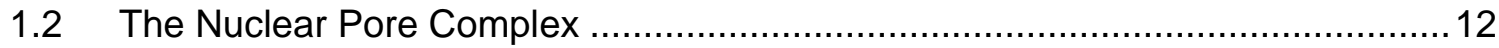

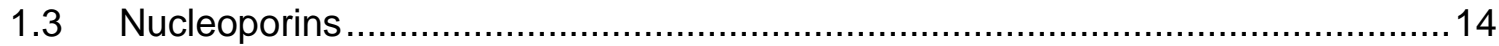

1.4 NPC Selectivity and Directionality of Transport ...................................... 15

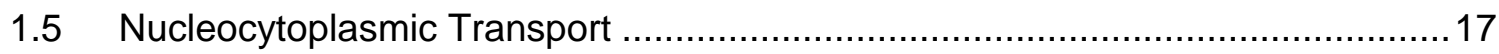

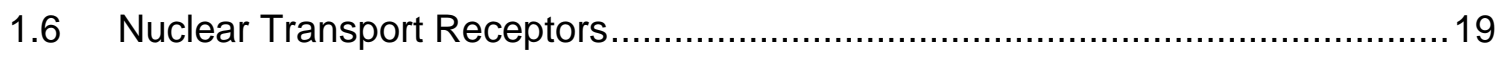

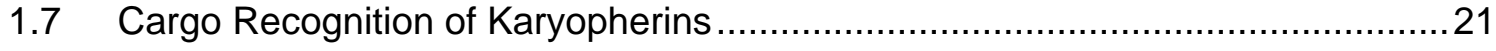

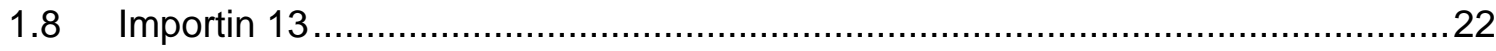

1.8.1 Importin 13 Cargo Recognition and Release ........................................25

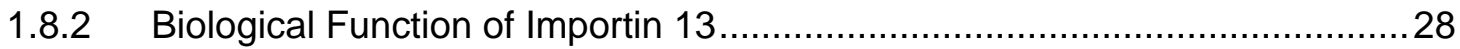

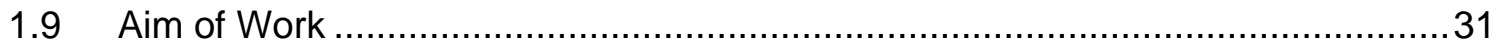

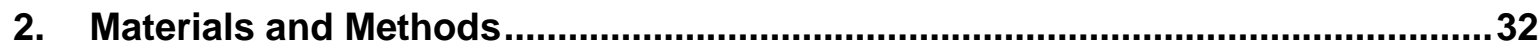

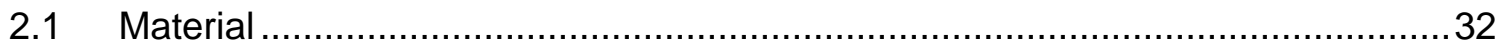

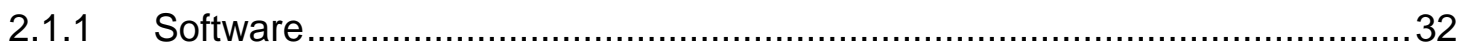

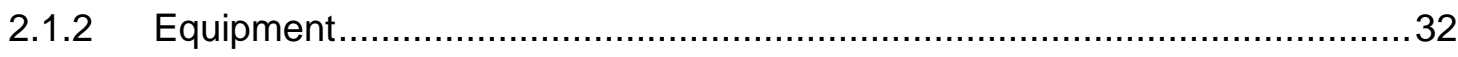

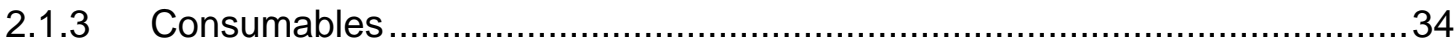

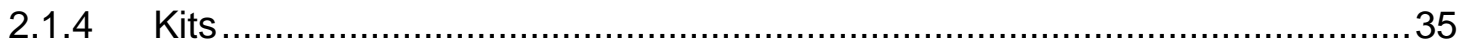

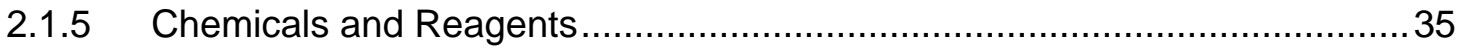

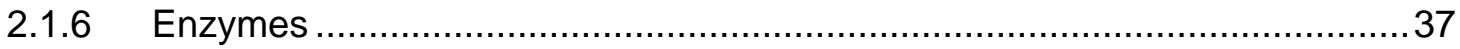

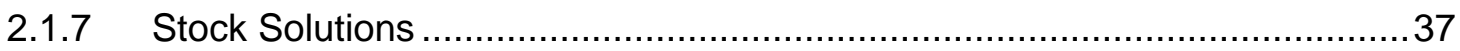

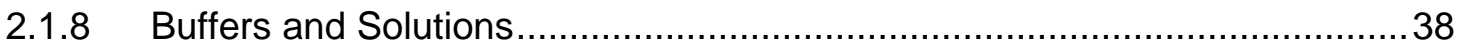

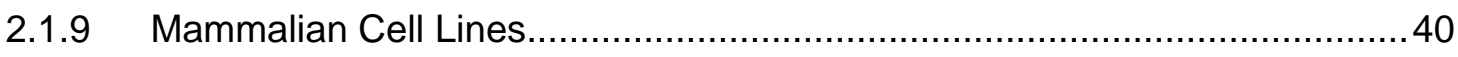

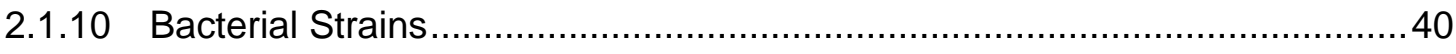

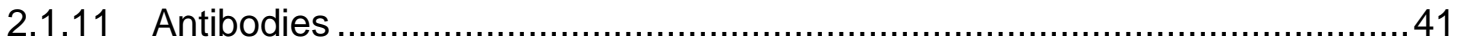

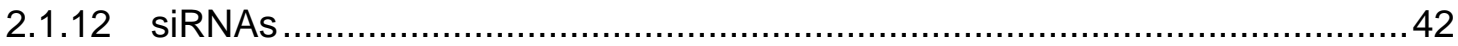

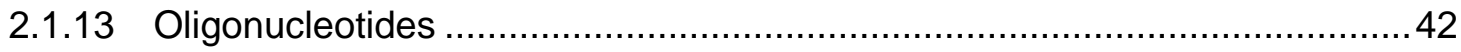

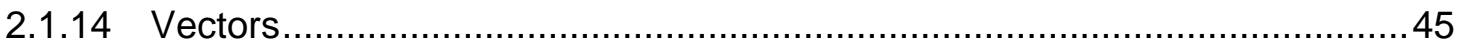

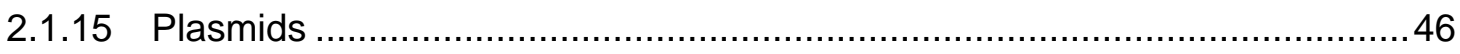

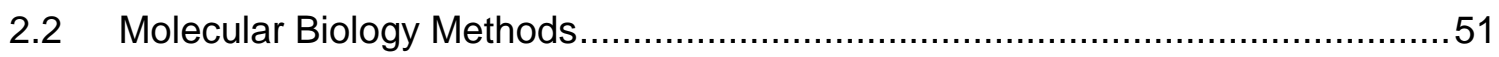

2.2.1 RNA Isolation from Cellular Extracts............................................... 51

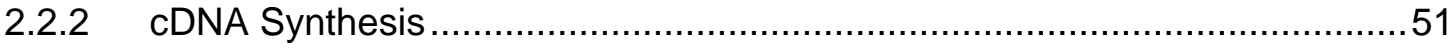




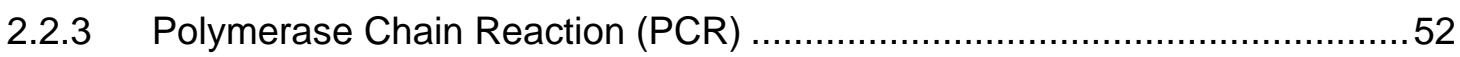

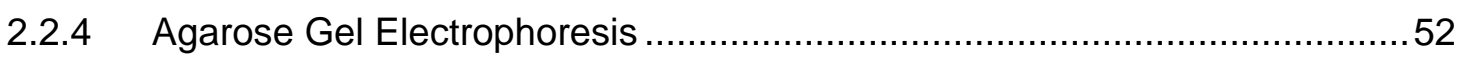

2.2.5 Purification of DNA Fragments from Agarose Gel .................................52

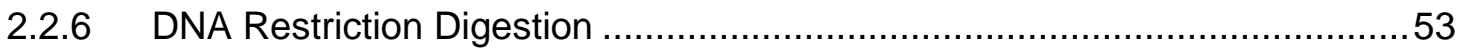

2.2.7 Dephosphorylation of Linearized Vectors ............................................ 53

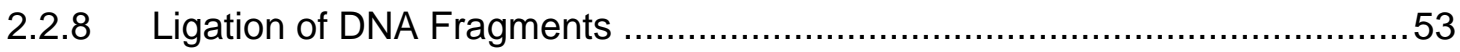

2.2.9 Transformation of $E$. coli with Plasmid DNA ..................................... 53

2.2.10 Small Scale Plasmid DNA Isolation ............................................... 54

2.2.11 Large Scale Plasmid DNA Isolation ............................................... 54

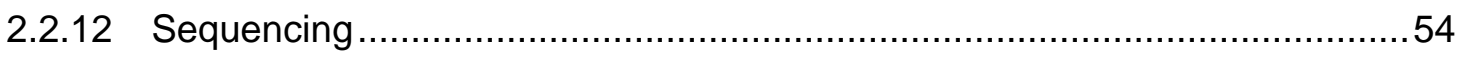

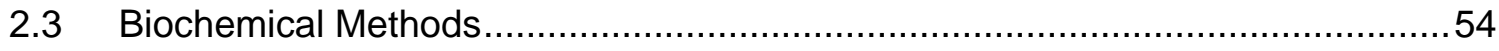

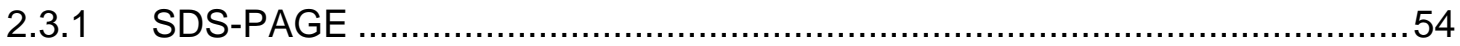

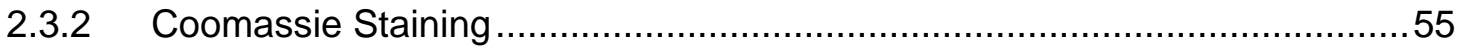

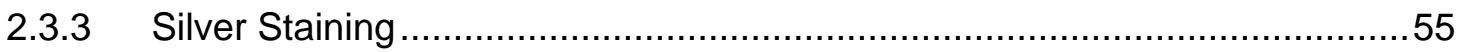

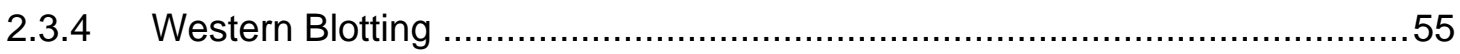

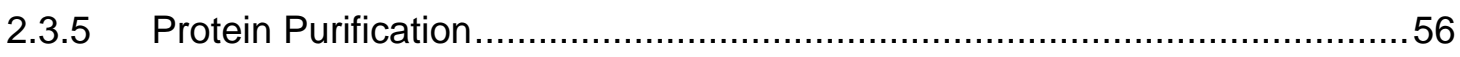

2.3.6 Protein Concentration Determination by Densitometry .............................59

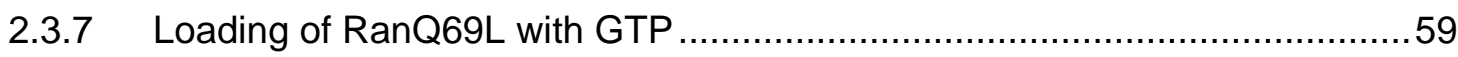

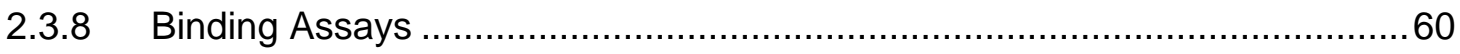

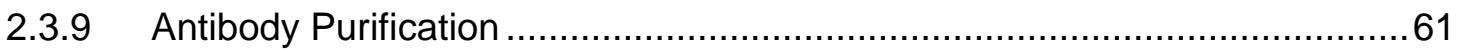

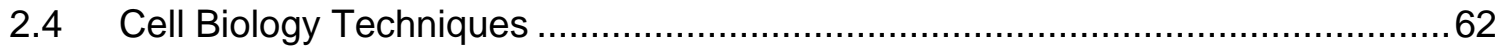

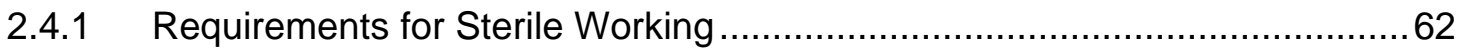

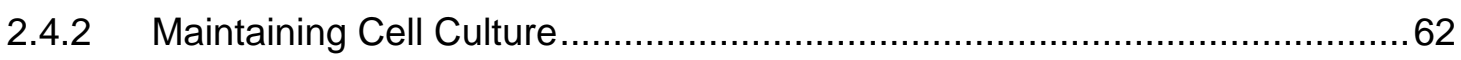

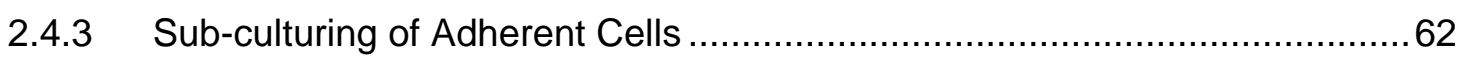

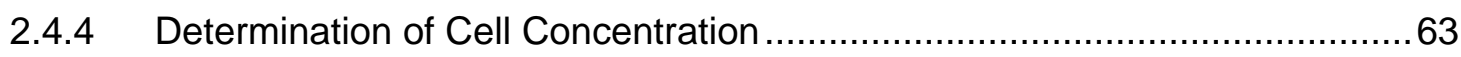

2.4.5 Coating of Cover Clips with Poly-L-Lysine ........................................... 63

2.4.6 Calcium Phosphate Transfection of Mammalian Cells ............................ 63

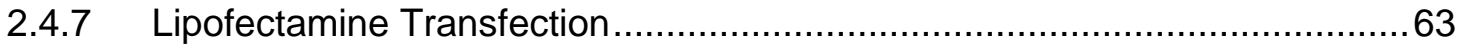

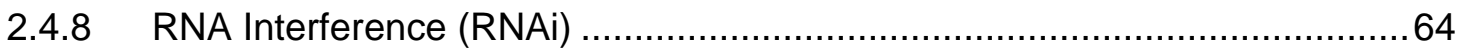

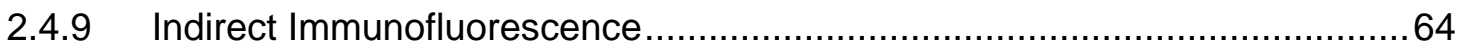

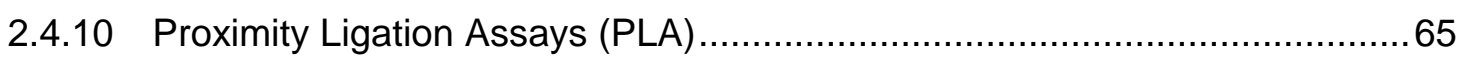

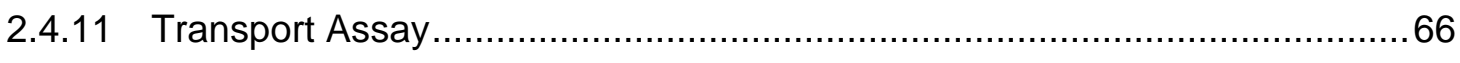

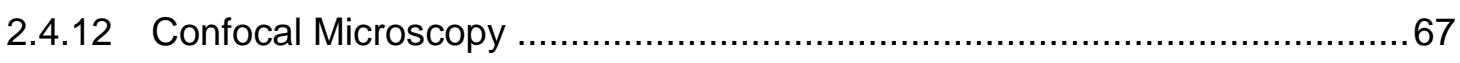

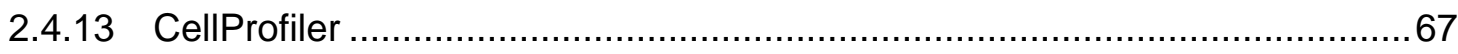




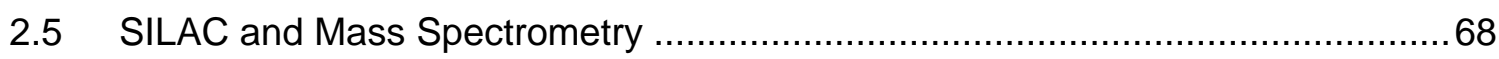

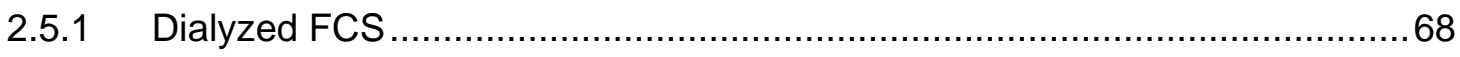

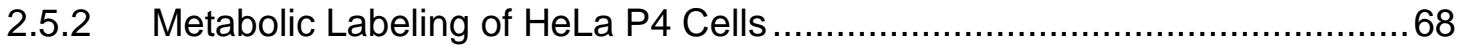

2.5.3 Binding Assay with Labeled HeLa P4 Cells ..............................................69

2.5.4 In-Gel Tryptic Digestion of Proteins .................................................... 70

2.5.5 Extraction of Peptides ................................................................... 71

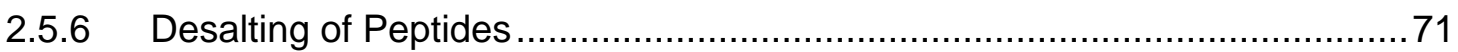

2.5.7 Liquid Chromatography-Coupled-Mass Spectrometry (LC-MS) Analysis of

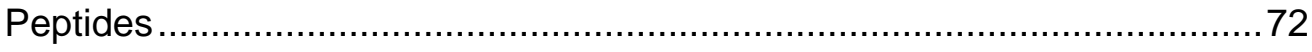

2.5.8 Analysis of Mass Spectrometry Data .............................................. 73

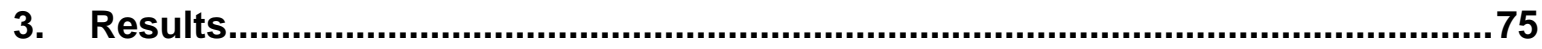

3.1 Characterization of Importin 13 and Established Cargoes ............................ 75

3.1.1 Importin 13 Expression Levels Are Low in Different Human Cancer Cell Lines .......................................................................................... 75

3.1.2 Importin 13 Is Rate Limiting in HeLa P4 Cells ..................................... 76

3.1.3 Importin 13 Affects the Subcellular Localization of elF1A and Ubc9 ..........77

3.1.4 Importin 13 Mediates Nuclear Import of Ubc9 In Vitro ...............................79

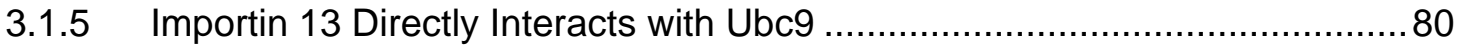

3.1.6 Importin 13 Binds Endogenous Ubc9 from HeLa P4 Cell Extracts ............... 82

3.2 Identification of Potential Importin 13 Export Cargoes Using an Importin 13

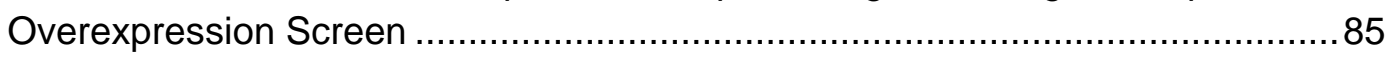

3.2.1 Importin 13 Overexpression Screen Using a Library of Nuclear Proteins..... 85

3.2.2 DBC-1, DMAP1, DDX43 and DDX59 Bind Importin 13 Differently to elF1A. 88

3.2.3 Characterization of DBC-1 Interaction with Importin 13........................... 90

3.2.3.1 Importin 13 Interacts with the Coiled-coil Domain of DBC-1 ................90

3.2.3.2 The N-terminal Domain of Importin 13 Is Required for Recognition of DBC-1 .......................................................................... 91

3.3 Identification of Potential Importin 13 Cargoes by Mass Spectrometry .............. 92

3.3.1 Mass Spectrometry Based Identification of Potential Importin 13

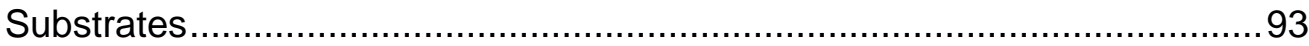

3.3.2 Identification of Single Importin 13 Bound Proteins Affected by Ubc9 or Enriched from HeLa P4 Cell Extract ...................................................96

3.3.3 Quantitative Mass Spectrometry Based Identification of Importin 13 Import and Export Cargoes Using SILAC ................................................. 100

3.3.3.1 Pull-down Based Identification of Potential Importin 13 Cargoes Using SILAC. 
3.3.3.2 SILAC Based Importin 13 Pull-downs Selectively Identify Importin 13 Cargoes

3.3.3.3 Filtering Criteria for the Identification of Importin 13 Import and Export Cargo Candidates.

3.3.3.4 Validation of Importin 13 Cargo Candidates Identified in SILAC Screen

3.3.3.4.1 Selection of Importin 13 Cargo Candidates for Further Analysis

3.3.3.4.2 Validation of Importin 13 Cargo Candidates Using Pull-down Experiments

3.3.3.4.3 Validation of Importin 13 Cargo Candidates in Overexpression Experiments

3.3.3.4.4 Characterization of the Interaction of Importin 13 with Importin 13

Cargo Candidates 139

3.3.3.4.5 Functional Roles of Novel Importin 13 Cargo Candidates 142

4. Discussion 144

4.1 Characterization of Known Importin 13 Cargoes 144

4.2 Overexpression Experiments for the Identification of Importin 13 Cargoes....... 145

4.2.1 Are DBC-1, DMAP1, TERT, DDX43 and DDX59 Importin 13 Cargoes? .... 145

4.2.2 Interaction of Importin 13 and DBC-1 147

4.2.3 Functional Link Between Importin 13 and Nup358 Mediated Transport? ... 148

4.3 Mass Spectrometry Based Identification of Importin 13 Cargoes 149

4.3.1 Mass Spectrometry Based Methods for the Identification of $\beta$-karyopherin Cargoes 149

4.3.2 SILAC Based Importin 13 Binding Assays for the Identification of Importin 13 Import and Export Cargoes.............................................. 149

4.3.2.1 Novel Importin 13 Export Cargoes................................................. 150

4.3.2.2 Novel Importin 13 Import Cargoes ............................................ 151

4.3.3 Importin 13 a Bidirectional Nuclear Transport Receptor of Many Import and Export Cargoes ................................................................... 154

4.3.4 Importin 13 a Negative Regulator of Nucleocytoplasmic Transport? .......... 154

4.3.5 Importin 13 an Exportin for M9 Containing Proteins? ............................. 155

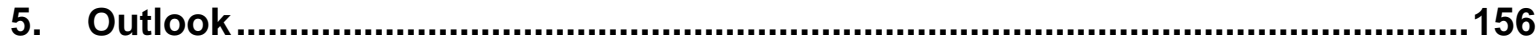

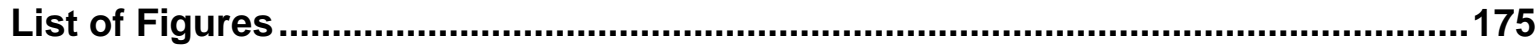

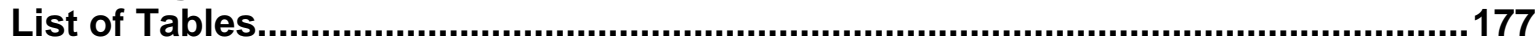

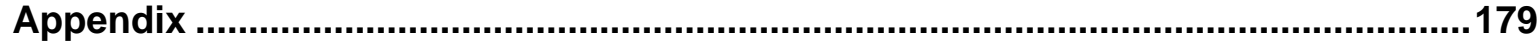

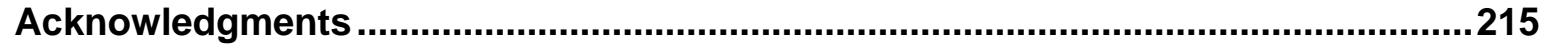




\section{Abstract}

Nuclear pore complexes embedded in the nuclear envelope regulate the bidirectional transport of macromolecules between the nucleus and the cytoplasm. Small molecules can rapidly move through the permeability barrier of the nuclear pore complex, whereas larger macromolecules typically require nuclear transport receptors to facilitate their diffusion. With more than 20 different transport receptors identified, only three have been reported to mediate both nuclear import and export, namely human exportin 4, yeast Msn5 and human importin 13. The latter was characterized as a bidirectional transport receptor in 2001 and since then several importin 13 import cargoes have been identified. For a long time, however, the translation initiation factor elF1A remained the only established export cargo. For a better understanding of the physiological significance of nuclear transport receptors and their diverse transport competencies, more cargoes need to be identified.

The central aim of this study was to expand the range of known importin 13 substrates using an importin 13 overexpression screen and a quantitative proteomics approach based on stable isotope labeling with amino acids in cell culture (SILAC). This approach should allow for the identification of proteins that bind to importin 13 in pull-down experiments under conditions that promote the formation of either import or export complexes.

In the overexpression screen, DBC-1, DMAP1, DDX43 and DDX59 were found to be redistributed to the cytoplasm upon importin 13 coexpression, identifying them as possible importin 13 substrates. Interestingly, transport of these proteins has previously been shown to be Nup358-dependent, suggesting a functional link between importin 13 and Nup358, possibly by Nup358 serving as an assembly or disassembly platform for importin 13 transport complexes. Detailed analysis of DBC-1 showed that its coiled-coil domain is required for interaction with importin 13.

In the SILAC based screen, more than 200 proteins were identified as potential importin 13 substrates, greatly expanding the repertoire of known cargoes for this transport receptor. Using importin 13 overexpression experiments, RTCA, FEN1, APEX1, SRP14, NSUN2, HNRNPD, XRCC5, BTF3, EIF2D, XRCC6 and SET were validated as potential importin 13 export cargoes, while ERI1 and NELFCD were identified as potential importin 13 import cargoes. In addition, importin 13 seems to function as an exportin for M9 signal sequence containing proteins.

Ultimately, the larger spectrum of importin 13 cargoes should give new insights into the physiological significance of importin 13 , its bidirectional transport competence, its unique mechanisms of cargo recognition and cargo release and, eventually, the identification of one or possibly even several conserved nuclear localization signals in cargo proteins. 


\section{Introduction}

\subsection{Intracellular Compartments}

The eukaryotic cell is subdivided into membrane-enclosed compartments or organelles, each containing their own functionally distinct subset of proteins and other molecules. One of the most prominent organelles is the nucleus, which contains the genome and is the principal site of DNA and RNA synthesis. The nucleus is encapsulated by the double membrane of the nuclear envelope, which physically separates the nucleus from the cytosol, the site of protein synthesis. This spatial separation of transcription and translation allows for more complex levels of gene expression as compared to prokaryotes that lack a membrane bound nucleus (1).

As the majority of proteins are synthesized in the cytosol, specific intracellular sorting signals and transport mechanisms are required that direct the proteins to their cellular compartments and allow for translocation of proteins across the organelle membranes. Proteins with functions in the nucleus such as histones, DNA and RNA polymerases, gene regulatory proteins and RNA processing proteins need to be selectively imported into the nuclear compartment, while at the same time tRNAs and mRNAs that are synthesized in the nucleus as well as ribosomal subunits need to be exported into the cytosol. Similarly, a resegregation of nuclear and cytoplasmic content is required upon nuclear envelope reassembly at the end of mitosis in mammalian cells (1).

There are three distinct mechanisms of protein trafficking between organelles, namely transmembrane transport, vesicular transport and gated transport. One example of gated transport, which will be further detailed in the following chapters, is the transport of RNA and proteins between the nucleus and the cytosol across the nuclear envelope through large macromolecular complexes, termed nuclear pore complexes (NPCs). NPCs serve as selective gates that allow active transport of specific macromolecules and macromolecular assemblies and free diffusion of smaller molecules (1).

\subsection{The Nuclear Pore Complex}

NPCs were initially observed by electron microscopy as pores within the nuclear envelope that later were shown to contain cylindrical formations $(2,3)$. The term "pore complex" was first assigned in 1959 (2) and since then advances in electron microscopy and other structural methods such as X-ray crystallography, mass spectroscopy and NMR spectroscopy have contributed significantly to the elucidation of the atomic structure of the 
NPC. Only recently, the first predictive structural model of the nuclear pore scaffold was presented by docking crystal structures of nucleoporins (proteins of the NPC) and nucleoporin complexes into a cryo-electron tomographic reconstruction of the intact human NPC (4-7). The core scaffold of the NPC is formed by three ring-like structures, the cytoplasmic ring, the central spoke ring and the nuclear ring, which surround the aqueous transport channel with a diameter of $\sim 60 \mathrm{~nm}(4-8)$ (Figure 1A). The central spoke ring is anchored in the nuclear envelope and connects the nuclear ring with the cytoplasmic ring. The cytoplasmic ring is decorated with eight cytoplasmic filaments, while a basket-like structure is connected to the nuclear ring where eight rod-like structures unite into a distal ring. Surrounding the central transport channel are eight smaller peripheral channels with a diameter of $\sim 9 \mathrm{~nm}$ at the narrowest point that have been suggested to allow diffusion of integral membrane proteins from the outer nuclear membrane to the inner nuclear membrane (9-11).
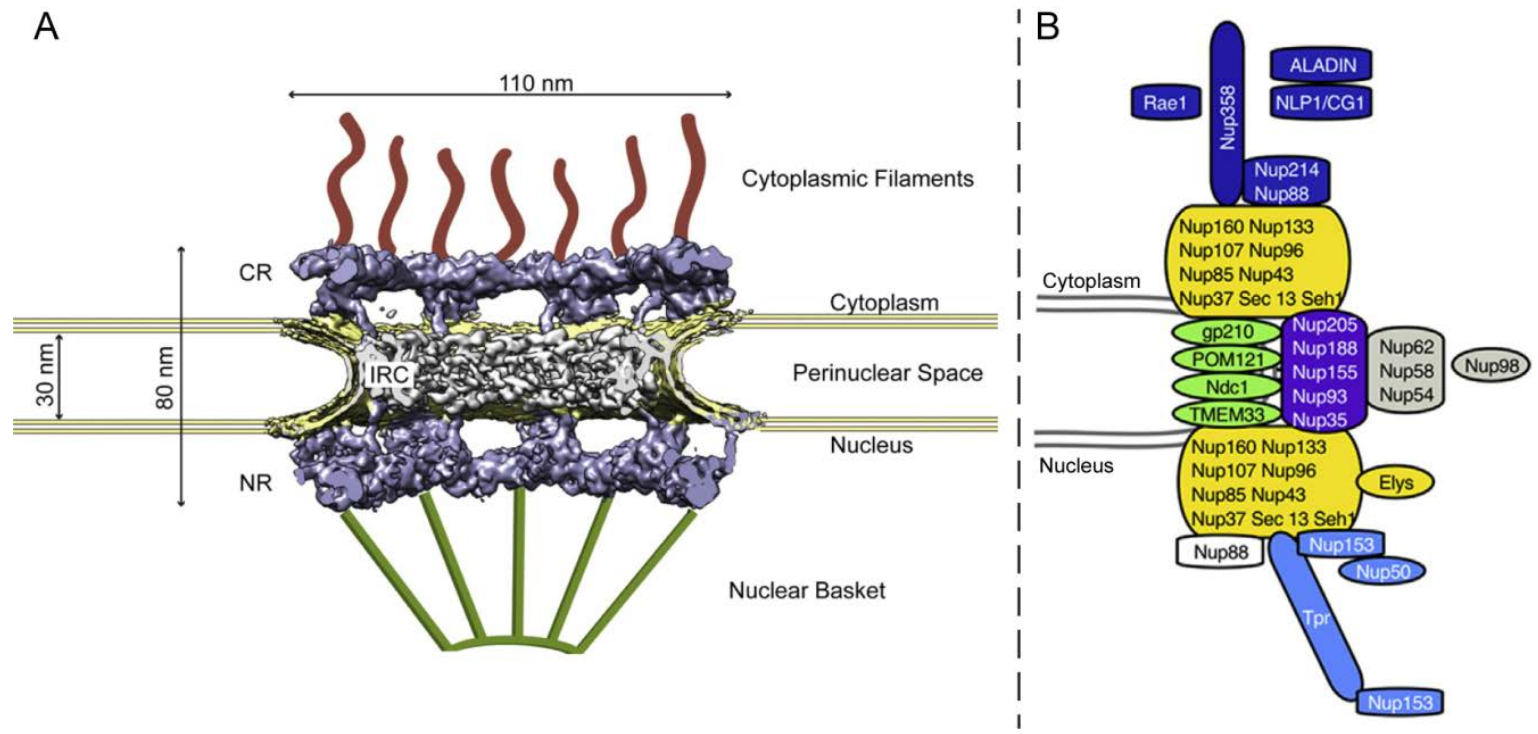

Figure 1: Structure of the nuclear pore complex. (A) Cryo-electron tomographic structure of the human NPC embedded in the nuclear envelope, decorated with schematic cytoplasmic filaments and the nuclear basket. CR: cytoplasmic ring; IRC: inner ring complex; NR: nucleoplasmic ring (modified from Schwartz 2016 (12)). (B) Structural organization and position of individual nucleoporins within the NPC (modified from Dickmanns et al., 2015 (13)).

NPCs show an eightfold rotational symmetry (14) along the nucleocytoplasmic axis and have a molecular mass of $\sim 112 \mathrm{MDa}(15)$, a diameter of $\sim 110 \mathrm{~nm}$ and a height of $\sim 80 \mathrm{~nm}$ (excluding cytoplasmic filaments and nuclear basket) in vertebrates (16). Structural 
features of the NPC are conserved between species even though its size ranges from $\sim 66 \mathrm{MDa}$ in yeast $(17,18)$ to $\sim 112 \mathrm{MDa}$ in vertebrates $(15)$. Furthermore, the density of NPCs in the nuclear envelope varies between different species with a typical mammalian cell containing 2,000-5,000 NPCs (19). Kinetic analysis of translocation through nuclear pore complexes suggests that up to 1,000 translocation events can occur per second per NPC, allowing a mass flow of nearly $100 \mathrm{MDa} / \mathrm{s}$ (20). Despite its gigantic dimensions, the NPC is composed of only $\sim 30$ different proteins, termed nucleoporins $(21,22)$. As nucleoporins occur in multiple copies, the fully assembled NPC consists of $\sim 500-1,000$ protein molecules (3).

\subsection{Nucleoporins}

Of the approximately thirty different nucleoporins (Nups) (Figure 1B) that constitute the NPC 20 nucleoporins are conserved among all eukaryotes, while the remaining -10 nucleoporins appear to be more specific to the different species (12). Historically, nucleoporins are named after their molecular weight but as this varies between different species no uniform nomenclature for nucleoporins exists (12). Instead, nucleoporins are classified into three different groups based on their amino acid sequence and predicted structural motifs, termed peripheral nucleoporins, scaffold nucleoporins and transmembrane nucleoporins (13). The transmembrane nucleoporins anchor the NPC to the nuclear envelope, the scaffold nucleoporins form a major part of the cytoplasmic and nuclear scaffold rings and the channel nucleoporins contain extensive natively unfolded phenylalanine-glycine repeats (FG-repeats) and form the permeability barrier of the NPC (13). Nucleoporins have been shown to organize into stable subcomplexes that include the Nup107 complex (also called Y complex), Nup93 complex, Nup62 complex and the Nup214 complex $(3,13)$. Further associated with the cytoplasmic ring is the Nup358-RanGAP1-SUMO1-Ubc9 complex that is implicated in the assembly and disassembly of transport complexes $(6,23-25)$.

Approximately a third of the nucleoporins contain intrinsically disordered FG-repeat regions that are believed to form the permeability barrier of the NPC and facilitate the selective transport of cargo through the pore by interacting with nuclear transport receptors (16). FG-nucleoporins contain FG stretches in multiples of 4 to 48 that are separated by spacer sequences of around 20, mainly polar, amino acids (19, 13). FG-domains are hydrophobic and characterized by an extreme depletion of charged residues. They can form either cohesive or non-cohesive interactions $(16,26)$. FG-nucleoporins both interact 
with constituents of the NPC scaffold and form homo- and heterotypical interactions with other FG-domains (26). The most common FG-repeat motifs are FG-, GLFG-, or FxFGrepeats $(13,26)$. The repetitive FG motifs mediate facilitated diffusion through the NPC by providing multiple low-affinity, high-specificity interactions with nuclear transport receptors (27). Even though much progress has been made in understanding the transport selectivity of NPCs, the exact gating mechanism remains unclear. Several transport models have been proposed that attempt to explain the selective barrier of NPCs (see section 1.4).

Apart from nucleocytoplasmic transport, nucleoporins are also involved in other cellular processes such as regulation of transcription, transcriptional memory, chromatin organization and DNA repair $(19,28,29)$. Dysregulation of nucleoporins can lead to the development of human diseases, such as cancer and certain genetic disorders $(13,30,31)$.

\subsection{NPC Selectivity and Directionality of Transport}

The NPC is freely permeable for small molecules, while larger molecules with a diameter of more than $\sim 5 \mathrm{~nm}$ or $\sim 30 \mathrm{kDa}$ in mass either take longer to cross the NPC or require nuclear transport receptors to facilitate their passage $(13,16)$. However, the permeability barrier of the NPC is not assumed to be perfect, also allowing the passage of proteins whose functions are purely cytoplasmic or nucleoplasmic (32). To avoid any harmful effects on cellular processes in the wrong cellular compartment, these proteins would either need to be inhibited or transported back into their designated compartment.

Several lines of evidence suggest that FG domains constitute the permeability barrier of the NPC (reviewed in (16)). In Saccharomyces cerevisiae, a reduced permeability barrier was observed upon genetic depletion of FG domains (26). Similarly, non-selective NPCs were observed upon nuclear assembly in Xenopus egg extracts depleted of specific FG-domains (33). Furthermore, purified FG domains have been shown to form FG-hydrogels with NPC-like properties that allow an influx of nuclear transport receptor-cargo complexes but prevent entry of large inert cargoes $(34,35)$. Despite the well-established requirement of FG-domains to confer transport selectivity, the precise composition of the FG-permeability barrier and translocation of nuclear transport receptors through the NPC is unknown. Various models have been proposed to explain the transport selectivity of NPCs, including the selective phase model (20), the virtual gate model (36), the reversible collapse model (37), the Kap-centric model (38), the forest-model (39) and 
the ring cycle model $(40,41)$. The models vary in the arrangement of FG-nucleoporins to confer a selective barrier and have been extensively discussed in several review papers $(16,42,43)$.

The virtual gate model (36) assumes that the central transport channel is densely covered by bristling, non-interacting FG-filaments that form an entropic barrier. Nuclear transport receptors can compensate for a loss in entropy with a change in enthalpy upon binding to the FG-domains of nucleoporins. The release of binding energy can then facilitate the entry of molecules into the crowded volume of the NPC, while inert molecules are excluded. The selective phase model (20) assumes that the FG-domains form intra- and intermolecular interactions resulting in a three-dimensional meshwork with sieve-like properties. Small molecules can diffuse through the meshwork, whereas molecules larger than the mesh-size would be excluded. Nuclear transport receptors can antagonize the cohesive inter-FG interactions by directly interacting with the hydrophobic FG-repeats, thereby partitioning the FG hydrogel, allowing the nuclear transport receptors to pass through the NPC. The forest model (39) is based on FG domains forming collapsed-coil and extended-coil conformations that can form cohesive or non-cohesive interactions, respectively. This results in two separate transport zones, a central route for macromolecules and a lateral route for small molecules.

The above transport models only consider the permeability barrier of the nuclear pore complex but do not address determinants of transport directionality. A well-established factor for directionality is the coupling of facilitated diffusion to the RanGTP gradient, with nuclear RanGTP concentrations being at least 200-fold higher than cytoplasmic concentrations $(44,45)$. The RanGTP gradient is established by the RanGTPase system and is discussed extensively in section 1.5. Further, peripheral nucleoporins often show high-affinity, RanGTP-sensitive binding of transport receptors that are believed to function as assembly or disassembly sites for transport complexes (for reviews see $(13,42)$ ). This observation is also part of the affinity gradient model that suggests that transport receptors show an increase in affinity for nucleoporins along the translocation pathway (46). A requirement for nucleoporins in the assembly of transport complexes has been shown, among others, for Nup358. Depletion of Nup358 in human cells resulted in a clear reduction of importin $\alpha / \beta$ and transportin-dependent transport that could only partially be restored by the addition of other transport factors $(47,48)$. This suggests that Nup358 is not absolutely required for nuclear import but facilitates the formation of transport complexes at the NPC by increasing the local concentration of transport receptors. Moreover, Nup358 was shown to interact directly with some cargoes such as DBC-1 
(deleted in breast cancer 1) and DMAP1 (DNA methyltransferase 1 associated protein 1), which might assist their interaction with nuclear import receptors and subsequent nuclear translocation (49).

\subsection{Nucleocytoplasmic Transport}

Transport between the nucleus and the cytoplasm across the nuclear envelope is mediated by transport machinery consisting of NPCs and the RanGTPase system as well as nuclear transport receptors that continuously shuttle between the nucleus and the cytoplasm (Figure 2). Nuclear transport receptors, which are divided into importins and exportins, bind their cargoes on one side of the nuclear envelope, cross the central channel of the NPC through interaction with FG-nucleoporins and release their cargo on the other side (16). To allow for an accumulation of the substrates against their chemical potential, an energy input is required that is usually derived from the RanGTPase system through GTP hydrolysis $(50,51)$. The RanGTPase system consists of the chromatin-bound guanine nucleotide exchange factor RCC1 (regulator of chromosome condensation 1) (52), the cytoplasmic filament-bound RanGTP activating protein (RanGAP) (53) and the small GTP-binding protein Ran (52). Due to the compartmentalization of RanGAP to the cytoplasm and of RCC1 to the nucleus, a steep RanGTP gradient is generated, with high nuclear RanGTP levels and low cytoplasmic levels $(50,51)$. RanGTP in turn actively regulates substrate binding and release by switching nuclear transport receptors between low- and high-affinity cargo-binding states $(16,54)$. RanGTP hydrolysis is not required for NPC translocation per se but for disassembly of transport complexes and consequently directionality of transport (43). 

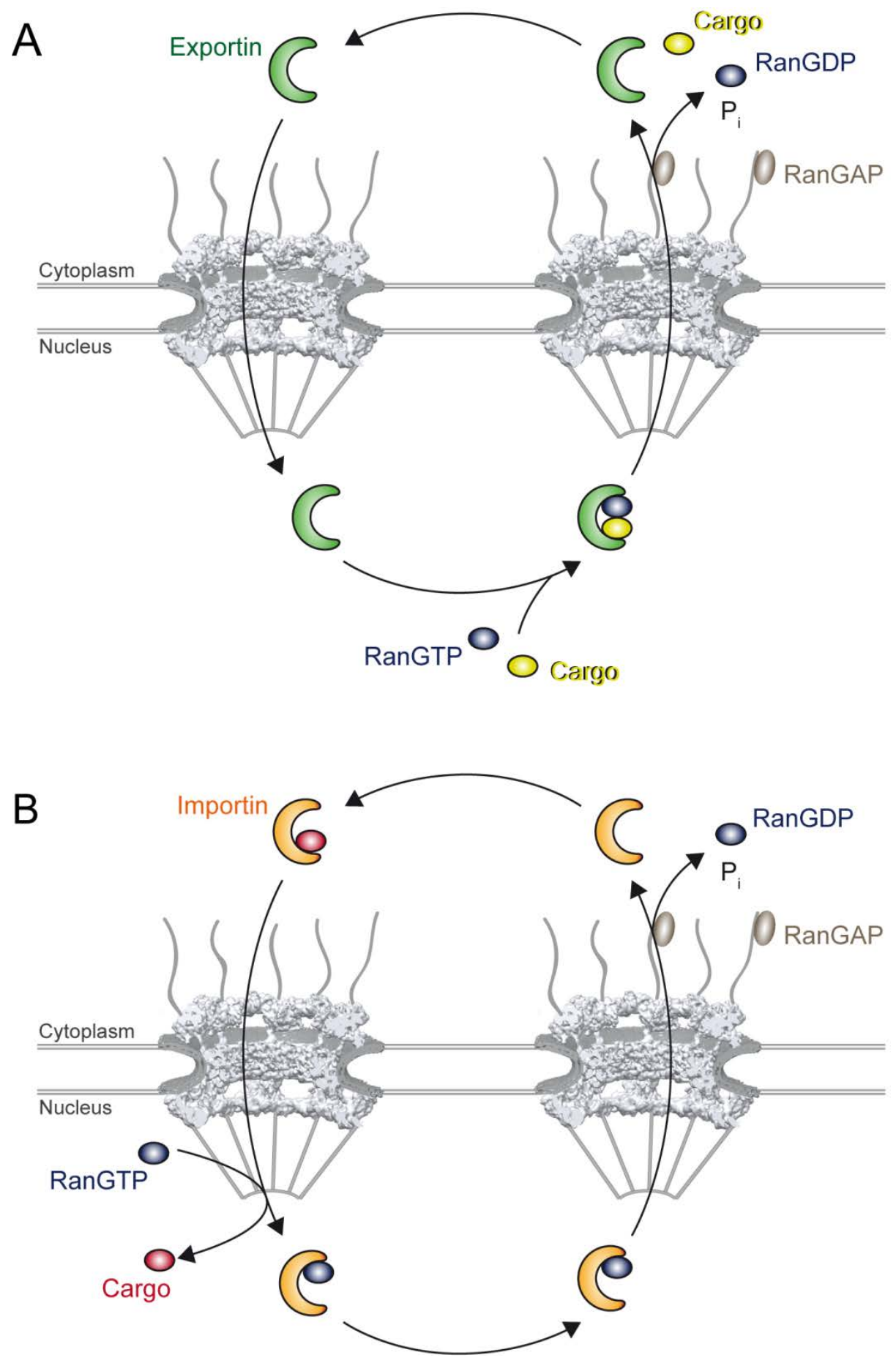

Figure 2: Nucleocytoplasmic transport. (A) Nuclear export. Exportins form a trimeric export complex with RanGTP and export cargo in the nucleus, shuttle through the nuclear pore complex and are disassembled in the cytoplasm by RanGAP mediated hydrolysis of GTP-bound Ran. Free exportins are recycled back to the nucleus for the next transport round. (B) Nuclear import. Importins bind their cargo in the cytoplasm, translocate across the nuclear pore complex and release the cargo in the nucleus upon RanGTP binding. Free importin bound to RanGTP is recycled back to the cytoplasm for the next transport round. The cryo-electron tomographic structure of the human NPC was taken from Appen et al., 2015 (6). 
Importins either directly bind their cargoes in the cytoplasm at low RanGTP concentrations or indirectly with the help of adaptor proteins. The import complex shuttles across the NPC via interactions with FG-nucleoporins and the cargo is released into the nucleus upon RanGTP binding $(54,55)$. The importin-RanGTP complex is then recycled back to the cytoplasm for subsequent transport rounds. In contrast, facilitated export in most cases requires the binding of exportins to both export cargo and RanGTP in a cooperative manner to form stable trimeric export complexes in the nucleus $(56,57)$. Export complexes can then translocate through the NPC into the cytoplasm, where they are disassembled upon GTP-hydrolysis on Ran. The intrinsic GTPase activity of Ran is promoted by RanGAP, assisted by soluble RanBP1 and NPC-bound Nup358 (also known as RanBP2) $(25,53,58,59)$. Following hydrolysis, free exportin and RanGDP in complex with its dedicated transport receptor, the nuclear transport factor 2 (NTF2), return to the nucleus (60-63), where RanGDP is converted back to RanGTP by the action of RCC1 $(52,64)$.

\subsection{Nuclear Transport Receptors}

In 1990, a study by Adam et al. (65) provided the first evidence that soluble transport factors are required for nuclear protein import. Through selective permeabilization of mammalian cells with digitonin, a reagent that permeabilizes the plasma membrane but leaves the nuclear envelope intact, they could show that nuclear accumulation of the SV40 large $\mathrm{T}$ antigen nuclear localization sequence fused to a fluorescent protein depends on the addition of exogenous cytosol. In subsequent studies, this assay allowed for the identification of several cytosolic proteins required for nuclear transport through fractionation of cytosol and testing the different fractions for nuclear import activity into the nuclei of digitonin permeabilized cells. The cytosolic transport proteins identified included importin $\alpha$, importin $\beta$, Ran and NTF2 (61, 60, 66-69).

Since these early studies, more than 20 different nuclear transport receptors have been identified that all share the ability to interact directly with FG-nucleoporins (43). Individual FG-nucleoporins bind to the convex outer surface of nuclear transport receptors through an interaction of FG-repeat domains with multiple hydrophobic patches on the nuclear transport receptor $(13,70)$. The interactions are transient and of low affinity, allowing for movement through the NPC $(20,54)$. Transport receptors are categorized into different classes based on conserved structural domains, including the importin $\beta$ protein family, the Mex67/TAP family and the NTF2 family $(13,43)$. By far the largest class is the superfamily of importin $\beta$-related proteins also known as $\beta$-karyopherins, named after the first transport 
receptor identified (43). This family of transport receptors recognizes nuclear localization signals and is responsible for most nucleocytoplasmic transport of proteins through the NPC (71). Even though $\beta$-karyopherins share a weak sequence homology of only $15-20 \%$, they show a similar overall structural organization containing $\sim 20$ HEAT-repeats that arrange into a superhelical or ring-like structure (72). The HEAT repeat, which consists of two antiparallel $\alpha$-helices connected by a short loop, is named after the proteins Huntingtin, elongation factor 3 (EF3), protein phosphatase 2A (PP2A) and the yeast PI3 kinase TOR1 where the structural motif was first identified $(73,74)$. This modular architecture gives $\beta$-karyopherins an intrinsic flexibility and allows for recognition of a wide range of different cargoes. Apart from the overall structural conservation, further unifying features of $\beta$-karyopherins are the similar molecular weights (90-150 kDa), an acidic isoelectric point ( $\mathrm{pl}=4.0-5.0)$ and the presence of an N-terminal RanGTP binding site at the inner concave surface (75).

$\beta$-karyopherins can be further divided into importins that shuttle proteins into the nucleus, exportins that shuttle proteins out of the nucleus and bidirectional transport receptors that mediate both nuclear import and export. In humans, ten $\beta$-karyopherins have been classified as importins (Impß, Trn1, Trn2, Trn-SR3, Imp4, Imp5, Imp7, Imp8, Imp9 and Imp11), seven as exportins (Crm1, CAS, Exp5, Exp6, Exp7, Expt and RanBP17) and two as bidirectional (Imp13 and Exp4) (76). In yeast, all nuclear transport receptors have been reported to sum up to a cellular concentration of $>10 \mu \mathrm{M}(77)$.

Until recently, only a limited number of cargoes had been identified for many nuclear transport receptors. Major advancements in mass spectrometry coupled with the use of stable isotope labeling with amino acids in cell culture (SILAC) $(78,79)$ allowed for identification of numerous transportin (80), importin $\beta$ (81), importin $\alpha / \beta$ (81) and Crm1 cargoes $(32,82)$, as well as cargoes of twelve import receptors in a study published this year (76). Only for some of these cargoes a consensus structure of the nuclear transport receptor binding site has been established.

As nucleoporins, nuclear transport receptors are not only involved in nucleocytoplasmic transport but also play a role in a range of other cellular processes such as mitosis and nuclear envelope assembly $(71,83-87)$, and are functionally regulated by protein modifications, inhibitory factors and specific anchorings (reviewed in (88)). 


\subsection{Cargo Recognition of Karyopherins}

Current crystal structures of transport complexes suggest that each $\beta$-karyopherin has multiple cargo binding sites. Nevertheless, the majority of importins seem to bind their cargoes at the concave inner surface of their C-terminus (89-100), whereas the major export receptor Crm1 appears to bind its cargoes at the convex outer surface of its C-terminus (101-104). For other exportins, only a limited number of crystal structures are available, making the identification of a potentially preferred binding site difficult (105-108). While most $\beta$-karyopherins associate directly with their cargoes, some also use adaptor proteins that bridge between the transport receptor and the targeting signal. One example is importin $\beta$, which uses one of the seven species of the importin a family $(109,110)$ as well as snurportin as an adapter for cargo binding (111), but also directly interacts with cargo (112). In addition to this, importin $\beta$ can also form a heterodimer with importin 7 and drive the nuclear import of the linker histone $\mathrm{H} 1(113,114)$.

Some cargoes bind to several $\beta$-karyopherins, suggesting a certain redundancy in cargo transport $(76,115-118)$. However, this finding is mainly based on in vitro studies and a preference for one particular transport receptor in vivo has been hypothesized (43). Nevertheless, some cargoes, especially larger cargoes, have been reported to require the simultaneous binding of multiple transport receptors to facilitate their transport (119).

Nuclear transport receptors are thought to bind their cargoes through nuclear localization signals, but consensus targeting signals have only been established for a few nuclear transport receptors (reviewed in $(71,120)$ ). The first targeting signal characterized was the 'classical' basic nuclear localization signal (cNLS) rich in lysines or arginines (121-123) that binds to importin $\alpha$ and is imported via the importin $\beta /$ importin $\alpha$ heterodimer $(66,124$, 125). Prototypic nuclear localization signals (NLSs) are the monopartite NLS in the SV40 $T$ antigen and the bipartite NLS in nucleoplasmin, which contain one or two clusters of positively charged amino acids $(121,123,122)$. The PY-NLS represent another class of NLSs that is recognized by transportin (91). The PY-NLS has an $R_{2-5} P Y$ motif at its C-terminus and either hydrophobic or basic motif at its N-terminus (91). Transportin-SR1 and its splice variant transportin-SR2 bind to RS domains that are rich in arginine-serine dipeptide repeats (126). The main export receptor Crm1 recognizes a short leucine-rich or hydrophobic nuclear export signal (NES) that was initially identified in HIV-1 Rev and protein kinase inhibitor A (127-130). For the other nuclear transport receptors, no consensus NLS has been described and their binding sites are not believed to be defined by a specific consensus sequence. Instead, they are ascribed by a number of physical properties such as intrinsic structural disorder, length, charge, hydrophobicity and spacing 
of key residues as well as three-dimensional conformations $(71,120)$. Ultimately, atomic structures of the transport complexes will be required to define more complicated and potentially three-dimensional binding sites.

As already discussed in section 1.5, cargo binding and release can occur via direct binding or through an allosteric mechanism and is often regulated by RanGTP binding. Cargo recognition is further regulated by the masking of the binding site through other proteins or nucleic acids, conformational changes in the binding site region or posttranslational modifications such as phosphorylation $(131,132)$.

\subsection{Importin 13}

The coding sequence of importin 13 was identified by Nagase et al. (133) in 1998 in a screen for cDNA clones from human brain coding for proteins larger than $50 \mathrm{kDa}$. The open reading frame of the importin 13 gene (IPO13), which they called KIAA0724, was mapped to chromosome 1 by radiation hybrid analysis. Sequence homology and motif searches against existing databases disclosed that importin 13 showed a sequence identity of less than $20 \%$ to known gene products at that time. A study in 2000 by Zhang et al. (134), identified KIAA0724 (termed LGL2) as a member of the $\beta$-karyopherin family, with the greatest homology to the transportin-SR subgroup. Nagase et al. in 1998 (133) had analyzed the expression pattern of importin 13 mRNA in ten different human tissues using reverse transcription-polymerase chain reaction coupled with an enzymelinked immunosorbent assay (RT-PCR ELISA). Importin 13 mRNA was present in all tissues tested with the highest expression levels present in the brain, followed by testis, heart, skeletal muscle, lung and kidney. Lower expression levels were detected in spleen, pancreas, liver and ovary.

The human protein encoded by KIAA0724 was analyzed in detail by Mingot et al. (135) in 2001. By searching Expressed Sequence Tags (EST) databases for sequences with significant homology to known members of the importin $\beta$ superfamily, they identified KIAA0724, which they termed importin 13 (IPO13 gene) and which codes for a human protein with 963 amino acids and a molecular weight of $108 \mathrm{kDa}$. Sequence homology analysis showed that importin 13 has putative orthologues in Arabidopsis thaliana, Drosophila melanogaster, Caenorhabditis elegans and Schizosaccharomyces pombe. In Schizosaccharomyces cerevisiae, no protein with a significant sequence homology is present, the closest orthologue being Pdr6p (also known as Kap122p). 
As a first confirmation that importin 13 might function as a nuclear transport receptor, Mingot et al., 2001 (135) showed that recombinant human importin 13 specifically interacts with RanGTP. By mass spectrometry they then identified several potential importin 13 substrates that bound to immobilized importin 13 from a HeLa cell extract in a RanGTP-dependent manner. Interestingly, most of the proteins identified, including 60S ribosomal protein L5, RNA-binding protein Y14 (referred to as RBM8,) protein mago nashi homolog (Mago, referred to as MGN), nuclear transcription factor $Y$ subunit beta (referred to as NF-YB) and SUMO-conjugating enzyme Ubc9, bound in the absence of RanGTP. Nup50 (referred to as NPAP), however, and eukaryotic translation initiation factor 1A (elF1A) bound efficiently only in the presence of RanGTP. Using binding assays and transport assays in digitonin permeabilized cells, Ubc9 and the Mago-Y14 protein complex were confirmed to be importin 13 cargoes, whereas elF1A was verified to be an export cargo, confirming the bidirectional transport capacity of importin 13.

Since the characterization of importin 13 in 2001, several importin 13 import cargoes have been identified in various studies (see Table 1). Only recently, two larger screens expanded the list of potential importin 13 substrates, using a stable isotope labeling with amino acids in cell culture (SILAC) based in vitro transport (SILAC-Tp) system, a method that employs nuclear import assays in digitonin permeabilized cells coupled with SILAC (76), and a yeast two-hybrid screen to identify interactors of the testis-specific form of importin 13, which lacks the N-terminus of importin 13 (amino acids 526-963) (136). However, for a long time, elF1A remained the only identified export cargo. Only at the end of 2016, eukaryotic translation initiation factor 4y2 (elF4G2) and high mobility group protein 20A (HMG20A) were also reported to be importin 13 export cargoes, underlining the bidirectional transport capacity of importin 13 (136). 
Table 1: Importin 13 import and export cargoes.

\begin{tabular}{|c|c|c|c|c|c|}
\hline Cargo & Function & Experiment & Position targeting signal & Karyopherins & Reference \\
\hline \multicolumn{6}{|c|}{ Importin 13 import cargoes } \\
\hline Ubc9 & SUMO E2-conjugating enzyme & binding assay, transport assay & folded domain & & $(93,135)$ \\
\hline $\begin{array}{l}\text { Mago/ Y14 } \\
(\mathrm{RBM} 8 \mathrm{~A})\end{array}$ & $\begin{array}{l}\text { core components of the exon junction } \\
\text { complex }\end{array}$ & binding assay, transport assay & folded domain & & $(92,135)$ \\
\hline Pax6 & paired homeodomain transcription factors & $\begin{array}{l}\text { yeast two hybrid, binding assay, } \\
\text { transport assay }\end{array}$ & $\begin{array}{l}\text { paired-type homeodomain } \\
\text { (aa 208-288) }\end{array}$ & & $(137)$ \\
\hline Pax3 & paired homeodomain transcription factor & binding assay, transport assay & paired-type homeodomain & Impa2 & (137) \\
\hline Crx & paired homeodomain transcription factor & binding assay, transport assay & paired-type homeodomain & & (137) \\
\hline Arx & paired homeodomain transcription factor & $\begin{array}{l}\text { yeast-two-hybrid, binding assay, } \\
\text { transport assay }\end{array}$ & paired homeodomain & $\operatorname{Imp} \beta$, Imp9 & $(138,139)$ \\
\hline $\mathrm{Nkx2-2}$ & transcriptional activator & binding assay, transport assay & homeodomain & $\operatorname{Imp} \beta$ & $(140)$ \\
\hline $\begin{array}{l}\text { NFYB/ } \\
\text { NFYC }\end{array}$ & component of transcriptional activator NFY & binding assay, transport assay & histone fold domain & & (141) \\
\hline C-Jun & part of the transcriptional complex AP-1 & binding assay, silPO13 & $\begin{array}{l}\text { basic region preceding } \\
\text { leucine zipper }\end{array}$ & $\begin{array}{l}\text { Imp } \beta \text {, transportin, } \\
\text { Imp7, Imp9 }\end{array}$ & $(117,142)$ \\
\hline GRL & $\begin{array}{l}\text { member of the nuclear receptor superfamily } \\
\text { of transcriptional regulators }\end{array}$ & $\begin{array}{l}\text { overexpression experiment, } \\
\text { binding assay, silPO13 }\end{array}$ & & $\operatorname{Impa/\beta ,~Imp7~}$ & (143) \\
\hline CAR & $\begin{array}{l}\text { member of the nuclear receptor superfamily } \\
\text { of transcriptional regulators }\end{array}$ & binding assay & ligand-binding domain & & (144) \\
\hline DBC-1 & multiple cellular functions & binding assay & & $\begin{array}{l}\text { Impa/ } \beta, \text { Imp7, } \\
\text { Imp9, transportin }\end{array}$ & (49) \\
\hline CTCF & transcriptional repressor & $\begin{array}{l}\text { binding assay; overexpression } \\
\text { experiment, silPO13 }\end{array}$ & middle region & & $(145)$ \\
\hline $\begin{array}{l}\text { NC2 } \alpha(\text { Drap1)/ } \\
\text { NC2 } \beta \text { (Dr1) }\end{array}$ & transcriptional regulator & $\begin{array}{l}\text { overexpression experiment, } \\
\text { binding assay }\end{array}$ & histone-fold domain & Impa/ $\beta$ & (146) \\
\hline $\begin{array}{l}\text { CHRAC15 } \\
\text { (CHRAC1)/ } \\
\text { CHRAC17 }\end{array}$ & $\begin{array}{l}\text { component of the chromatin accessibility } \\
\text { complex }\end{array}$ & $\begin{array}{l}\text { overexpression experiment, } \\
\text { transport assay }\end{array}$ & histone-fold domain & & $(135,147)$ \\
\hline p12/CHRAC17 & component of polymerase $\varepsilon$ & $\begin{array}{l}\text { overexpression experiment, } \\
\text { transport assay }\end{array}$ & histone-fold domain & & $(135,147)$ \\
\hline Myopodin & actin-bundling protein & $\begin{array}{l}\text { yeast-two hybrid, binding assay, } \\
\text { silP013 }\end{array}$ & C-terminal portion & Impa & (148) \\
\hline $\mathrm{ARH} 1$ & tumor suppressor & binding assay & & Impa, Imp7, Imp9 & (149) \\
\hline \multicolumn{6}{|c|}{ Importin 13 export cargoes } \\
\hline elF1A & translation initiation factor & binding assay, transport assay & folded domain & & $(107,135)$ \\
\hline EIF4G2 & translation initiation factor & binding assay, IPO13 knock-out, & & & (136) \\
\hline HMG20A & transcriptional regulator & $\begin{array}{l}\text { binding assay, IPO13 knock-out, } \\
\text { overexpression experiment, FRAP }\end{array}$ & & & (136) \\
\hline
\end{tabular}


Apart from importin 13, the only nuclear transport receptors that have been characterized as bidirectional transport receptors are exportin 4 and yeast Msn5 (150, 151). Yeast Msn5 was shown to export the inhibitor of G1 cyclin-Cdk complex Far1p and transcription factors Swi5p, Swi6p, Msn2p and Pho4p while also importing the trimeric replication protein $A$ (RPA), which is required for multiple aspects of DNA metabolism, including DNA replication, DNA repair and recombination (151-156). Human exportin 4 exports the translation initiation factor elF5A, the intracellular signal transducer and transcriptional modulator Smad3 and the thyroid hormone receptor but was also shown to import the sex-determining region $Y$ protein $(S R Y)(157,158)$ and the transcription factor Sox2 (150, 157-159). Both SRY and Sox2 are Sox family members and are imported through their high-mobility group (HMG), which is hypothesized to be recognized as a threedimensional structure and not as a linear signal sequence (150). Interestingly, exportin 4 has also been reported to interact with the transcription factor Sox9 via its high-mobility group domain in the absence of RanGTP and to modulate its DNA binding activity but not to affect its intracellular localization (160).

In contrast to other karyopherins, bidirectional nuclear transport receptors can shuttle not only one, but two cargoes per hydrolysis of one GTP molecule through the NPC (135). However, this lesser expenditure of energy is suggested to limit the extent of cargo accumulation and therefore bidirectionality is not believed to be a universal transport mechanism of karyopherins (135). Instead, several karyopherins transport some of their cargoes as homodimers, heterodimers or even larger complexes. One example is importin 13, which was shown to shuttle the heterodimers Mago-Y14 (core components of the exon junction complex), NC2 $\alpha / N C 2 \beta$ (transcriptional regulation), CHRAC15/CHRAC17 (part of a chromatin remodeling complex), p12/CHRAC17 (integral component of DNA-polymerase $\varepsilon$ ) and NFYB/NFYC (part of the transcriptional activator NFY) across the nuclear envelope $(135,141,146,147)$. Importin 13 is not only distinctive in its ability to mediate both nuclear import and export but also appears to be differentially regulated compared to other nuclear transport receptors in terms of cargo recognition and transport complex disassembly.

\subsubsection{Importin 13 Cargo Recognition and Release}

Similar to other $\beta$-karyopherins, importin 13 folds into 20 consecutive HEAT repeats, a motif that consists of two antiparallel $\alpha$-helices (three parallel $\alpha$-helices for the last repeat) with inter-repeat and intra-repeat loops protruding to opposite sides $(92,93,107)$. The 
superhelical structure of importin 13 is highly flexible, allowing for a whole range of importin 13 conformations, from a tight ring-like structure to a wide, open superhelix $(92,93,107)$. Apo-importin 13 and importin 13 when bound to the import cargoes Mago/Y14 and Ubc9 have a more extended conformation, while importin 13 bound to Ran and the export cargo elF1A, or Ran alone, has a more compact conformation $(92,93,107)$.

Crystal structures of importin 13 in complex with Ubc9, the heterodimer Mago/Y14 and elF1A/Ran (Figure 3) as well as binding assays suggest that importin 13 recognizes and also releases its cargoes via different mechanisms (92, 93, 107, 135). Importin 13 does not bind its cargoes through a linear sequence or through a small portion of its cargoes, as reported for importin $\beta$, transportin and Crm1, but instead seems to recognize its cargoes via folded domains and several conserved charged and polar residues distributed over the entire protein $(92,93,107)$. An example are the homeodomain containing cargo proteins, such as Pax6 and Crx, which require cooperativity between two basic clusters located at the $\mathrm{N}$-terminus and the $\mathrm{C}$-terminus of their homeodomain, in order to be imported by importin $13(137,161,140)$.

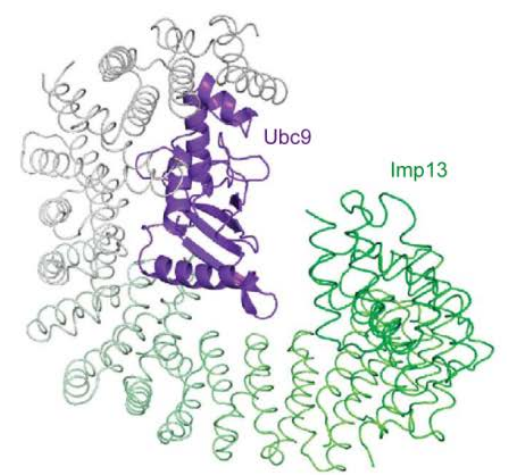

Importin 13 - Ubc9

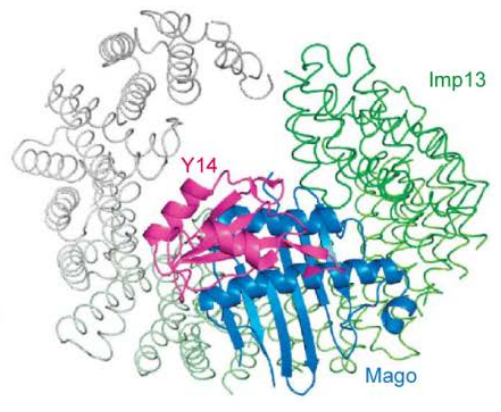

Importin 13 - Mago-Y14

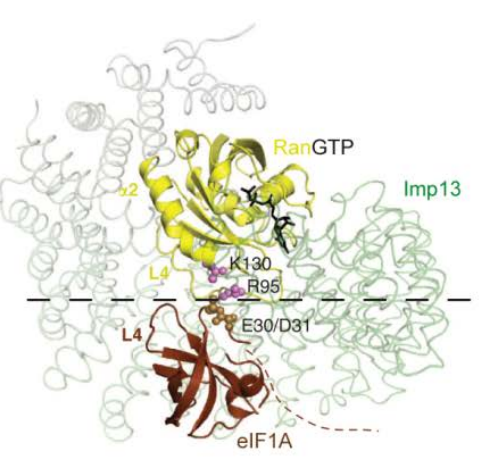

Importin 13 - RanGTP - elF1A

Figure 3: Structures of importin 13 import and export complexes. Importin 13 is shown as a ribbon trace, with a color gradient from grey (N-terminus) to green (C-terminus). Bound molecules are shown as cartoons, with Ubc9 in purple, Mago in blue, Y14 in magenta, Ran in yellow, GTP in black and eIF1A in brown (modified from Grünwald et al., 2011 (93) and 2013 (107)).

Importin 13 binds its import cargoes Ubc9 and Mago/Y14 at its inner concave surface at non-overlapping sites, with Mago/Y14 binding to the C-terminal arch of importin 13 shifted 
towards the inter-repeat loops rather than the intra-repeat loops of the HEAT motif, and Ubc9 binding to the $\mathrm{N}$-terminal half of importin 13 shifted towards the intra-repeat loops $(93,107)$. This binding mode of Ubc9 is rather unusual as most importin structures solved to date suggest that importins bind their cargoes primarily through their C-terminal arch $(89,91,94-100,111)$. An exception is the parathyroid hormone-related protein (PTHrP), which binds in a highly extended conformation to the HEAT repeats 2-11 of importin $\beta$, largely overlapping its RanGTP binding site (90).

Comparable to other karyopherins, Ran in its GTP-bound form binds to the inner concave surface of importin 13 shifted towards the intra-repeat loops of the HEAT motif and contacts two highly conserved sites at an N-terminal (HEAT 1-3) and a central region of importin 13 (HEAT 8-9) as well as a less conserved region at its C-terminus (HEAT 16-19) (92). While RanGTP contacts HEAT 14 and 15 in importin $\beta$ and yeast Cse1 (human orthologue is CAS), it contacts HEAT 16-19 in importin 13 and Crm1, resulting in a more closed conformation of the later karyopherins (92).

In contrast to other karyopherins, importin 13 likely does not bind its export cargo elF1A through cooperative binding with RanGTP. Instead, RanGTP binding seems to facilitate the formation of the export complex by displacing bound import cargo $(107,135)$. However, it should be noted that elF1A has been suggested to engage in a stabilizing contact with RanGTP when bound to importin 13 (107). Importin 13 recognizes elF1A at its inner surface, shifted towards the inter-repeat loops of the HEAT motif, through two major binding sites that spatially overlap with the Mago/Y14 binding site (107). The larger interaction surface can be found in the middle region of importin 13, while the smaller interaction surface is located to its very C-terminus (107). Although the crystal structures suggest that a concomitant binding of elF1A and Ubc9 as well as Mago/Y14 and Ubc9 would theoretically be possible, binding experiments show that simultaneous binding does not occur $(93,107)$.

As a consequence of the different cargo binding sites, importin 13 has different mechanisms for cargo release. In contrast to other karyopherins, importin 13 lacks the acidic loop that is utilized by importin $\beta$ (HEAT 8), transportin (HEAT 8) and Crm1 (HEAT 9) for cargo release (92). Instead, importin 13 cargo release into the nucleus is mediated by RanGTP binding through both a steric hindrance mechanism and a direct competition mechanism for the same binding surface $(92,93)$. The direct competition mechanism is employed by the import cargo Ubc9, as RanGTP binds to the same binding site as Ubc9 (93), while Mago/Y14, which docks to a binding site adjacent to RanGTP, is released due to steric clashes of the two proteins (92). High nuclear concentrations of 
RanGTP as well as its higher affinity for importin 13 compared to the import cargoes, efficiently facilitates import cargo release into the nucleus (107). In vitro, however, efficient Mago/Y14 release depends not only on RanGTP binding but also requires the presence of the importin 13 export cargo elF1A, suggesting that elF1A locks importin 13 in an export complex and prevents reassociation of Mago/Y14. Interestingly, similar to Mago/Y14, hydrolysis of RanGTP is likely not sufficient to disassemble the elF1A export complex in vitro. Additionally, the loading of a tightly binding import cargo is required to fully displace the export cargo eIF1A from importin $13(107,135)$.

\subsubsection{Biological Function of Importin 13}

Importin 13 has been reported to be expressed in various tissues in a cell type- and differentiation stage-specific manner, and to play a role in the embryonic development of lung, brain and heart, while its deregulation has been linked to human diseases. Importin 13 regulates its own expression via a positive feedback mechanism, mediating nuclear import of CCCTC-binding factor (CTCF), a multivalent zinc-finger protein that binds to the IPO13 promoter and induces expression of importin 13 (145).

The expression of importin 13 is developmentally regulated in rat fetal lung, human limbal epithelial basal cells and mouse fetal brain. In rat fetal lung, importin 13 expression is hormonally regulated by glucocorticoids and importin 13 is enriched in epithelium relative to the mesenchyme (134). Importin 13 mRNA levels are most abundant during the pseudoglandular stage of lung development (Days 14-16) and decrease during the canalicular (Day 18) and saccular (Day 20) stages (134). Interestingly, not only the expression level of importin 13 is developmentally regulated in rat fetal lung but also its nucleocytoplasmic shuttling, with importin 13 entering the nucleus much more rapidly at fetal Day 18 than at Day 21 (162). This suggests a role of importin 13 in normal lung embryogenesis by possibly mediating nuclear import of transcription factors (162). Indeed, it could be shown that importin 13 regulates nuclear import of the glucocorticoid receptor in airway epithelial cells, which regulates the transcription of genes involved in development, metabolism and immune response (143). This could be of relevance for anti-inflammatory asthma therapy, as glucocorticoids are critical to the treatment of asthma and other airway inflammations (143). Genetic variations of importin 13 have been shown to be associated with improved airway responsiveness in childhood asthma (163).

Importin 13 is solely expressed in human limbal epithelial cells, not in other cell layers of the limbus (border of the cornea and the white of the eye) and was shown to play an 
important role in maintaining the undifferentiated phenotype and high proliferation potential of corneal epithelial progenitor cells (164). Increased importin 13 activity is associated with the pathogenesis of pterygium, a triangular wing-shaped overgrowth of abnormal conjunctiva onto the cornea (142). Overexpression or knock-down of importin 13 increased or decreased pterygium epithelial cell proliferation, respectively (142).

Expression and subcellular distribution of importin 13 are also regulated during brain development in mice, with the highest expression levels in fetal brain tissues at mouse embryonic day 13.5 (E13.5) and then gradually decreasing, with the lowest expression in adult mouse brain tissues (165). In the telencephalon (embryonic structure from which the cerebrum develops prenatally) tissue at stage E11.5 endogenous importin 13 is mainly localized in the cytoplasm, while at later stages from E15.5 to P0, importin 13 is mainly located in the nucleus (165). Further support for a potential role of importin 13 in neural development comes from a study (166) showing that importin 13 regulates neurotransmitter release at the Drosophila neuromuscular junction and that some of the identified importin 13 substrates are important in embryonic neural development. Pax6, for example, is a master control for eye morphogenesis $(137,167,168)$ and Arx is necessary for development of the forebrain $(138,169,170)$.

During mouse development, importin 13 expression increases significantly from fertilized egg to blastocysts (171). Furthermore, meiotic differentiation of mouse germ cells is influenced by the stage-specific activity of importin 13 (172). Importin 13 is expressed in the primordial germ cells in the mouse embryo and is later expressed predominantly at the pachytene phase of meiosis in both male and female germ cells (172). Knock-down of importin 13 in fetal oocytes impedes the progression of meiosis through the pachytene phase of prophase I (172). In the same study, the authors identified a shorter transcript of mouse importin 13, which is encoded by the IPO13 gene but utilizes a different transcription start site (172). This shorter transcript is identical to the C-terminal fragment of importin 13 but lacks the N-terminal RanGTP-binding site and was shown to be only expressed in the germ cells in the adult testis (172). Expression of this shorter importin 13 testis-specific transcript prevented nuclear localization of Ubc9 in GC1 cells derived from spermatogenic cells, whereas expression of full-length importin 13 resulted in a primarily nuclear localization of Ubc9. The authors suggest that this short importin 13 transcript may act as a dominant negative regulator of importin 13 mediated nuclear import (172). This function was further confirmed in a study (136), where they identified the interactome of the testis-specific form of importin 13. In the same study, the authors also demonstrated 
that the short importin 13 transcript may not only play a role in the germ cells of the testis, but also in mature spermatozoa (136).

Differential expression of importin 13 has also been linked to cancer, with increased importin 13 expression in endometrial carcinoma compared to secretory endometrium (173).

Even though many of the cargoes known to be regulated by importin 13 have been linked to cellular functions, much remains to be investigated to understand how importin 13 affects specific cellular states. For this, not only will more cargoes need to be identified, but also importin 13 expression regulation, its effect on cargo subcellular localization and consequently specific cellular pathways will need to be analyzed in detail and ultimately linked. Another aim will be to understand how deregulation of any of these individual processes can lead to pathogenic alterations of cellular states. 


\subsection{Aim of Work}

Importin 13 is one of the few transport receptors known to function both in nuclear import and export. While comprehensive lists of potential transportin, importin $\alpha / \beta$ and $\mathrm{Crm} 1$ cargoes were available (80-82), only a few importin 13 cargoes had been reported when this work was started. The aim of this thesis was therefore to expand the range of importin 13 substrates using three different approaches.

The first approach focused on the specific identification of importin 13 export cargoes. For this, nuclear proteins derived from the 'LIFE database' $(174,175)$, were expressed in HeLa cells and their subcellular localization was analyzed upon coexpression of importin 13. In the second approach, potential substrates from a HeLa cell extract, which interact specifically with immobilized importin 13 , were identified by mass spectrometry. The third approach was similar to the second approach but was designed to allow the distinction between import and export cargoes using quantitative proteomics. To this end, stable isotope labeling of amino acids in cell culture (SILAC) was applied, to identify proteins that specifically interact with immobilized importin 13 from a HeLa cell extract. Finally, identified cargo candidates should be validated in binding assays and importin 13 overexpression experiments.

Together, these approaches should lead to a better understanding of importin 13-dependent nuclear transport. 


\section{Materials and Methods}

\subsection{Material}

\subsubsection{Software}

Adobe Illustrator CS7

AxioVision LE64 4.9.1.0

CellProfiler 2.1.1

Citavi 5

DAVID Bioinformatics Resources 6.8

GraphPad Prism 5.01

Image Reader LAS-3000

ImageJ/Fiji

Lasergene 10.1.1 (3)

LSM 510 Release Version 4.0 SP2

LSM Image Browser

MaxQuant 1.5.1.0

Microsoft Office 2010

NanoDrop 2000 Software

Perseus 1.5.0.15

PPT Drawing Toolkits

Proteome Discoverer 1.4

SnapGene Viewer 3.1.4

STRING 10.0

Tm Calculator

Venny 2.1 .0

\subsubsection{Equipment}

Agarose gel documentation GelSTICK touch

Agarose gel running chamber

Acclaim $^{\text {TM }}$ PepMap $^{\text {TM }} 100$ pre-column
Adobe

Zeiss

Broad Institute

Swiss Academic Software

$\mathrm{NIH}$

GraphPad Software Inc.

Fujifilm

$\mathrm{NIH}$

DNASTAR

Zeiss

Zeiss

Max Planck Institute of

Biochemistry

Microsoft

Thermo Scientific

Max Planck Institute of

Biochemistry

Motifolio Inc.

Thermo Scientific

GSL Biotech LLC

STRING Consortium

Thermo Scientific

Centro Nacional de

Biotecnología

INTAS Science Imaging

Instruments

Home-made, Workshop, UMG

Thermo Scientific 
ÄKTA column HiLoad 26/60 Superdex 200 prep grade

ÄKTA column HiLoad 26/60 Superdex 75 prep grade

ÄKTA column MonoS 5/50 GL

ÄKTA column Superdex 200 10/300 GL

ÄKTA column Superdex 75 10/300 GL

ÄKTApurifier

Autoclave DX-200

BioPhotometer

CASY 1

Cell culture hood Herasafe ${ }^{\mathrm{TM}} \mathrm{KS}$

Cell culture incubator Heracell ${ }^{\mathrm{TM}}$ 150i

Cell culture incubator Cytoperm 2

Centrifuge 5415R

Centrifuge 5424

Centrifuge Sigma 1-15

Centrifuge Allegra ${ }^{\circledR}$ X-15R with rotor SX4750

Centrifuge Allegra ${ }^{\circledR}$ X 22 with rotor SX4250

Centrifuge Avanti ${ }^{\mathrm{TM}} \mathrm{J}-30 \mathrm{I}$ with rotor JA30.50Ti

Centrifuge J6-MI with rotor JS 4.2

Centrifuge Optima MAX-XP with rotor TLA100.3

Confocal microscope LSM 510 META

Decon FS-100 ultrasonic bath

Developer machine CURIX60

Dual Gel Caster for Mini Vertical Units

EmulsiFlex-C3

Fluorescence microscope Axioskop 2

Incubator Heraeus function line

Incubator Shaker INNOVA 4430

Incubation/Inactivation Water Bath Model 1003

Mini Trans-Blot ${ }^{\circledR}$ Cell

Olympus CK40 Culture Microscope

Orbitrap Velos Pro ${ }^{\mathrm{TM}}$ Nano ESI Mass Spectrometer

SE250 Mighty Small II Mini Vertical Electrophoresis

Unit

SDS gel documentation LAS-3000
GE Healthcare

GE Healthcare

GE Healthcare

GE Healthcare

GE Healthcare

Amersham Biosciences

Systec

Eppendorf

Schärfe System

Thermo Scientific

Thermo Scientific

Heraeus Instruments

Eppendorf

Eppendorf

Sigma-Aldrich

Beckman Coulter

Beckman Coulter

Beckman Coulter

Beckman Coulter

Beckman Coulter

Zeiss

Decon Laboratories

Agfa

Hoefer

Avestin

Zeiss

Heraeus

New Brunswick Scientific

GFL

Bio-Rad

Olympus

Thermo Scientific

Hoefer

Fujifilm 
Spectrophotometer NanoDrop 2000c

SpeedVac Concentrator

Thermocycler FlexCycler2

Thermocycler PTC-200 DNA Engine

Thermocycler Tprofessional

Thermomixer comfort

Thermomixer compact

UltiMate $^{\mathrm{TM}} 3000$ RSLCnano System

UV sterilizer

UV transilluminator

Vacuum Christ Alpha 1-4

Vortexer MS2 Minishaker

XCell SureLock ${ }^{\circledR}$ Mini-Cell

\subsubsection{Consumables}

$5 \mathrm{~mL}$ Polystyrene Round-Bottom Tubes

Amersham Hybond ECL Nitrocellulose Blotting

Membrane

Amersham Hyperfilm ${ }^{\mathrm{TM}} \mathrm{ECL}$

Amersham Protran $0.45 \mu \mathrm{m}$ NC Nitrocellulose Blotting

Membrane

Cell culture consumables

Cell culture plastic ware

Centrifuge Bottle Assembly, Polycarbonate $50 \mathrm{~mL}$

Centrifuge tube, thickwall, Polycarbonate $500 \mu \mathrm{L}$

Casy cups with lids

Empore $^{\mathrm{TM}} \mathrm{C} 1847$ mm Extraction Disc, Model 2215

LC-MS Screw Neck Vial, $1.5 \mathrm{~mL}$

LC-MS vial insert, $250 \mu \mathrm{L}$

Medix XBU medical x-ray film

Microscope cover slips (10 mm, $12 \mathrm{~mm} \varnothing$ )

Microscope slides

Mini-Protean ${ }^{\circledR}$ TGX Gels
Thermo Scientific

Savant

Analytik Jena AG

MJ Research

Biometra

Eppendorf

Eppendorf

Thermo Scientific

Biometra

Uvitec

W. Krannich

IKA

Life technologies

BD Biosciences

GE Healthcare

GE Healthcare

GE Healthcare

Sarstedt, Greiner bio-one

Sarstedt, Greiner bio-one, Nagle

Nunc International

Beckman Coulter

Beckman Coulter

Roche Diagnostics (Fisher

Scientific)

3M Company

Grace

Agilent Technologies

FOMA Bohemia

Marienfeld

Thermo Scientific

Bio-Rad 
Minisart RC 15, single use syringe filters $(0.45 \mu \mathrm{m}$, $0.20 \mu \mathrm{m})$

Minisart single use filter units $(0.45 \mu \mathrm{m}, 0.20 \mu \mathrm{m})$

NuPAGE ${ }^{\circledR}$ Novex $^{\circledR} 4-12 \%$ Bis-Tris Protein Gels

Parafilm "M"

PD-10 columns

$\mathrm{pH}$ indicator strips

Protein LoBind Tubes

Reaction tubes $(1.5,2 \mathrm{~mL})$

Spectra/Por ${ }^{\circledR}$ Dialysis Membrane

Spin- $X^{\circledR}$ UF Concentrator

Syringes and needles

Whatman gel blotting paper

\subsubsection{Kits}

CloneJET PCR Cloning Kit

Duolink $^{\circledR}$ In Situ Detection Reagents Red

Duolink $^{\circledR}$ In Situ Kit

NucleoBond ${ }^{\mathrm{TM}}$ Xtra Midi

NucleoSpin ${ }^{\circledR}$ Gel and PCR Clean-up

NucleoSpin ${ }^{\circledR}$ Plasmid

Pierce ${ }^{\circledR}$ BCA Protein Assay Kit

Pierce ${ }^{\circledR}$ Silver Stain Kit

RNeasy Mini Kit
Sartorius Stedim Biotech

Sartorius Stedim Biotech

Thermo Scientific

Bemis Company, Inc.

GE Healthcare

Macherey-Nagel

Eppendorf

Sarstedt, greiner bio-one

Spectrum Laboratories

Corning

B. Braun, Servoprax

GE Healthcare

Thermo Scientific

Sigma-Aldrich

Sigma-Aldrich

Macherey-Nagel

Macherey-Nagel

Macherey-Nagel

Thermo Scientific

Thermo Scientific

Qiagen

\subsubsection{Chemicals and Reagents}

All standard chemicals and solvents not listed here were obtained from Applichem GmbH (Darmstadt), Carl Roth GmbH + Co. KG (Karlsruhe), Serva Electrophoresis GmbH (Heidelberg), Sigma-Aldrich (Taufkirchen) or Merck (Darmstadt).

Arg-6: $\mathrm{HCl}$

Arg-10: $\mathrm{HCl}$

Lys-4D:2HCl

Lys-8: $\mathrm{HCl}$

$\beta$-Mercaptoethanol
Silantes

Silantes

Silantes

Silantes

Roth 
Acetonitrile, Optima ${ }^{\mathrm{TM}}$ LC/MS Grade

Acrylamide 4K Solution (30\%)

Adenosine 5'-triphosphate disodium salt hydrate

(order number A3377)

Ammonium hydrogen carbonate

Bovine Serum Albumin (BSA) $(20 \mathrm{mg} / \mathrm{mL})$

BSA, fraction $\mathrm{V}$

Coomassie Plus ${ }^{\mathrm{TM}}$ Protein Assay Reagent

Coulter ISOTON ${ }^{\circledR}$ II Diluent

Cyanogen bromide-activated Sepharose 4B

Dako Fluorescence Mounting Medium

DAPI (D9542)

DEAE-Sepharose

Digitonin

dNTP Set, $100 \mathrm{mM}$ solutions

FBS Superior

Formaldehyde solution min. $37 \%$

Formic Acid, 99.5+\%, Optima ${ }^{\text {TM }}$ LC/MS Grade

Gelatin from cold water fish

GeneRuler 100bp DNA Ladder

GeneRuler 1kb DNA Ladder

Gibco $^{\circledR}$ DMEM (1x)

Gibco $^{\circledR}$ DMEM (1x) no glutamine, lysine \& arginine

Gibco $^{\circledR}$ L-Glutamine

Gibco $^{\circledR}$ Opti-MEM $^{\circledR}(1 \mathrm{x})$

Gibco ${ }^{\circledR}$ Penicillin Streptomycin (Pen Strep)

Glutathione Sepharose 4 Fast Flow

Glutathione Sepharose High Performance

Guanosine 5'-diphosphate sodium salt (G7127)

Guanosine 5'-triphosphate sodium salt hydrate (51120)

IgG-Sepharose 6 Fast Flow

Immobilon ${ }^{\mathrm{TM}}$ Western Chemiluminescent HRP

Substrate

Iodoacetamide, BioUltra

Isopropyl $\beta$-D-1-thiogalactopyranoside
Fisher Scientific

Applichem

Sigma-Aldrich

Roth

Thermo Scientific

Applichem

Thermo Scientific

Beckman Coulter

Sigma-Aldrich

Dako

Sigma-Aldrich

Sigma-Aldrich

Calbiochem

Thermo Scientific

Biochrom

Millipore

Fisher Scientific

Sigma-Aldrich

Thermo Scientific

Thermo Scientific

Thermo Scientific

Thermo Scientific

Thermo Scientific

Thermo Scientific

Thermo Scientific

GE Healthcare

GE Healthcare

Sigma-Aldrich

Sigma-Aldrich

GE Healthcare

Millipore

Sigma-Aldrich

Thermo Scientific 
Lectin from Triticum vulgaris

Leptomycin B Enzo

L-Glutathione reduced

Lipofectamine ${ }^{\circledR} 2000$

Methanol, Optima ${ }^{\text {TM }}$ LC/MS Grade

MOWIOL $^{\circledR}$ 4-88

Ni-NTA Agarose

NUPAGE ${ }^{\circledR}$ MES SDS Running Buffer (20x)

NuPAGE ${ }^{\circledR}$ MOPS SDS Running Buffer (20x)

Oligofectamine $^{\mathrm{TM}}$ Reagent

Oligonucleotides

ortho-Phosphoric acid 85\% p.A.

PageRuler Prestained Protein Ladder

PageRuler Unstained Protein Ladder

Poly-L-Lysine solution 0.1\% (w/v)

SafeView ${ }^{\mathrm{TM}}$ Classic (DNA stain)

SP-Sepharose Fast Flow

Water, Optima ${ }^{\mathrm{TM}}$ LC/MS Grade

\subsubsection{Enzymes}

Creatine phosphokinase, Rabbit Skeletal Muscle

DNasel

Fast alkaline phosphatase (FastAP)

Phusion $^{\circledR}$ High-Fidelity DNA Polymerase

Restriction enzymes

RiboLock RNase Inhibitor

SuperScript ${ }^{\circledR}$ III Reverse Transcriptase

T4 DNA ligase

Gibco $^{\circledR}$ Trypsin/ EDTA 0.25\% (1x)

Trypsin NB Sequencing Grade (peptide digestion)
Sigma-Aldrich

Life Sciences

Applichem

Thermo Scientific

Fisher Scientific

Calbiochem

Qiagen

Thermo Scientific

Thermo Scientific

Thermo Scientific

Sigma-Aldrich

AppliChem

Thermo Scientific

Thermo Scientific

Sigma-Aldrich

Applied Biological Materials Inc.

Roth

Fisher Scientific

Calbiochem

Roth

Thermo Scientific

Thermo Scientific

Thermo Scientific

Thermo Scientific

Thermo Scientific

Thermo Scientific

Thermo Scientific

Serva

\subsubsection{Stock Solutions}

\section{1,4-Dithiothreitol (DTT)}

Ammonium persulfate (APS)
$1 \mathrm{M}$ in $\mathrm{H}_{2} \mathrm{O}$

$10 \%$ APS (Sigma) in $\mathrm{H}_{2} \mathrm{O}$ 
Ampicillin

Aprotinin

Adenosine triphosphate (ATP)

Calcium chloride buffer

Chloramphenicol

Creatine phosphokinase

Creatine phosphate

Cytosol $(9 \mathrm{mg} / \mathrm{mL})$

Digitonin

FITC-Phalloidin

Hoechst 33258

Isopropyl b-D-1-thiogalactopyranoside

(IPTG)

Kanamycin

Leupeptin/Pepstatin

Phenylmethylsulfonyl fluoride (PMSF)

WGA (wheat germ agglutinine/lectin)
$100 \mathrm{mg} / \mathrm{mL}$ in $\mathrm{H}_{2} \mathrm{O}$

$1 \mathrm{mg} / \mathrm{mL}$ in $20 \mathrm{mM}$ HEPES $\mathrm{pH} 7.4$

$100 \mathrm{mM}$ ATP in $100 \mathrm{mM} \mathrm{Mg}(\mathrm{OAc})_{2}, 20 \mathrm{mM}$

HEPES (pH 7.4)

$250 \mathrm{mM} \mathrm{CaCl}_{2} \mathrm{H}_{2} \mathrm{O}$

$34 \mathrm{mg} / \mathrm{mL}$ in EtOH

$2000 \mathrm{U} / \mathrm{mL}$ in 50\% glycerol, $20 \mathrm{mM}$ HEPES

$\mathrm{pH} 7.4$

$80 \mathrm{mg} / \mathrm{mL}$ in $\mathrm{H}_{2} \mathrm{O}$

prepared as described in Kehlenbach et al., 1998

$10 \%(w / v)$ in DMSO

$0.2 \mathrm{mg} / \mathrm{mL}$ in methanol

$10 \mathrm{mg} / \mathrm{mL}$ in $\mathrm{H}_{2} \mathrm{O}$

$1 \mathrm{M}$ in $\mathrm{H}_{2} \mathrm{O}$

$50 \mathrm{mg} / \mathrm{mL}$ in $\mathrm{H}_{2} \mathrm{O}$

$1 \mathrm{mg} / \mathrm{mL}$ each, in DMSO

$100 \mathrm{mM}$ in 2-propanol

$2 \mathrm{mg} / \mathrm{mL}$ in TPB

\subsubsection{Buffers and Solutions}

2YT-medium

Carbonate buffer

Coomassie destaining solution

Coomassie fixation solution

Coomassie staining solution

DNA loading buffer (6x)

GST buffer
$1.6 \%(\mathrm{w} / \mathrm{v})$ tryptone, $1 \%(\mathrm{w} / \mathrm{v})$ yeast extract, $0.5 \%(\mathrm{w} / \mathrm{v}) \mathrm{NaCl}, \mathrm{pH} 7.0$

$0.2 \mathrm{M} \mathrm{NaHCO}_{3} / \mathrm{Na}_{2} \mathrm{CO}_{3}, \mathrm{pH} 8.9$

$10 \%$ acetic acid

$40 \%$ ethanol, $10 \%$ acetic acid

$5 \%$ aluminum sulfate-(14-18)-hydrate, 10\%

ethanol, $2 \%$ ortho-phosphoric acid, $0.02 \%$

CBB-G250

$0.2 \%$ bromophenol blue, $0.2 \%$ xylene cyanole, $60 \%$ glycerol, $60 \mathrm{mM}$ EDTA

$50 \mathrm{mM}$ Tris pH 6.8, $300 \mathrm{mM} \mathrm{NaCl}, 1 \mathrm{mM} \mathrm{MgCl}$, $2 \mathrm{mM}$ DTT, $0.1 \mathrm{mM}$ PMSF, $1 \mu \mathrm{g} / \mathrm{mL}$ of each AP and LP 
HBS (2x) buffer

His buffer

Laemmli running buffer (10x)

LB-medium

LB agar plates

Mowiol mounting medium

PBS (10x)

PBS-T

PLA Wash Buffer A

PLA Wash Buffer B

PonceauS staining solution

Pulldown buffer

Ran buffer

SDS sample buffer (4x)

SOC-medium

STOP buffer

TAE buffer (50x)

Transport buffer (10x)

Ubc9 buffer

50 mM HEPES, $250 \mathrm{mM} \mathrm{NaCl}, 1.5 \mathrm{mM}$

$\mathrm{Na}_{2} \mathrm{HPO}_{4}, \mathrm{pH} 6.98$

$50 \mathrm{mM}$ Tris pH 7.5, $500 \mathrm{mM} \mathrm{NaCl}, 10 \mathrm{mM}$

MgAc, 5\% glycerol, $10 \mathrm{mM} \beta$-mercaptoethanol, $0.1 \mathrm{mM}$ PMSF, $1 \mu \mathrm{g} / \mathrm{mL}$ of each AP and LP $250 \mathrm{mM}$ Tris, $1.92 \mathrm{M}$ glycine, 0.5\% SDS $1 \%(\mathrm{w} / \mathrm{v})$ bacto-tryptone, $0.5 \%(\mathrm{w} / \mathrm{v})$ yeast

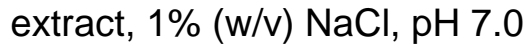
LB supplemented with 1.5\% (w/v) bacto-agar 13.3\% (w/v) Mowiol 4-88, 33.3\% (w/v) glycerol, $133 \mathrm{mM}$ Tris- $\mathrm{HCl}, \mathrm{pH} 8.5,1 \mu \mathrm{g} / \mathrm{mL}$ DAPI $1.37 \mathrm{M} \mathrm{NaCl}, 27 \mathrm{mM} \mathrm{KCl}, 100 \mathrm{mM} \mathrm{Na}_{2} \mathrm{HPO}_{4}$, $18 \mathrm{mM} \mathrm{KH}_{2} \mathrm{PO}_{4}, \mathrm{pH} 7.5$

$1 x$ PBS $+0.1 \%$ Tween-20

$0.01 \mathrm{M}$ Tris pH 8.0, 0.15 M NaCl, $0.05 \%$

Tween 20

$0.2 \mathrm{M}$ Tris $\mathrm{pH} 7.5,0.1 \mathrm{M} \mathrm{NaCl}$

$0.5 \%$ Ponceaus in $1 \%$ acetic acid

$50 \mathrm{mM}$ Tris pH 7.4, $200 \mathrm{mM} \mathrm{NaCl}, 5 \%$ glycerol, 2 mM DTT

$50 \mathrm{mM}$ Tris pH 8.0, $75 \mathrm{mM} \mathrm{NaCl}, 1 \mathrm{mM} \mathrm{MgCl}_{2}$, $100 \mu \mathrm{M}$ PMSF, $1 \mu \mathrm{g} / \mathrm{mL}$ of each AP and LP $125 \mathrm{mM}$ Tris pH 6.8, 4\% SDS, $0.02 \%$ Bromophenol blue, 10\% glycerol $2 \%(\mathrm{w} / \mathrm{v})$ tryptone, $0.5 \%(\mathrm{w} / \mathrm{v})$ yeast extract, $10 \mathrm{mM} \mathrm{NaCl}, 2.5 \mathrm{mM} \mathrm{KaCl}, 10 \mathrm{mM} \mathrm{MgCl}_{2}$, $10 \mathrm{mM} \mathrm{MgSO}_{4}, 0.36 \%$ (w/v) glucose, $\mathrm{pH} 7.0$ $7 \%$ charcoal, $10 \%$ ethanol, $0.1 \mathrm{M} \mathrm{HCl}, 10 \mathrm{mM}$ $\mathrm{NaH}_{2} \mathrm{PO}_{4}$

$2 \mathrm{M}$ Tris, $0.05 \mathrm{M}$ EDTA, 5.71\% acetic acid 200 mM HEPES, 1.1 M KOAc, 20 mM $\mathrm{Mg}(\mathrm{OAc})_{2}, 10 \mathrm{mM}$ EGTA, pH 7.3 $50 \mathrm{mM}$ Na-phosphate pH 6.5, $50 \mathrm{mM} \mathrm{NaCl}$, $0.1 \mathrm{mM}$ PMSF, $1 \mu \mathrm{g} / \mathrm{mL}$ of each AP and LP, $1 \mathrm{mM}$ DTT 
Western blot transfer buffer (10x)

Western blot transfer buffer (1x)
$250 \mathrm{mM}$ Tris, $1.93 \mathrm{M}$ glycine, 0.2\% SDS

$10 \%$ Western blot transfer buffer (10x), 20\%

$\mathrm{MeOH}$

\subsubsection{Mammalian Cell Lines}

\begin{tabular}{lll}
\hline Cell line & Specification & Origin \\
\hline HeLa P4 (P4 MAGI & Human adenocarcinoma cell line that & NIH AIDS Reagent \\
CCR5+ Cells) & $\begin{array}{l}\text { expresses CD4; derived from cervix of } \\
\text { a 31-year-old woman }\end{array}$ & Program; (176) \\
U2-OS (U2OS) & $\begin{array}{l}\text { Human osteosarcoma cell line; derived } \\
\text { from a moderately differentiated }\end{array}$ & ATCC ${ }^{\circledR}$ HTB96 \\
& $\begin{array}{l}\text { sarcoma of the tibia of a 15-year-old girl } \\
\text { Human embryonic kidney cell line }\end{array}$ & ATCC, CRL-2828 \\
& $\begin{array}{l}\text { transformed with adenovirus 5 DNA; } \\
\text { derived from fetus }\end{array}$ & \\
COS-7 & Monkey African green kidney cell line & Sigma-Aldrich, \\
& transformed with SV40 & 87021302 \\
\hline
\end{tabular}

\subsubsection{Bacterial Strains}

\begin{tabular}{|c|c|}
\hline Bacterial strain & Specification and Genotype \\
\hline BL21 (DE3) codon+ & $\begin{array}{l}\text { F- ompT hsdS(rB- mB-) dcm+ Tetr gal I (DE3) endA Hte [argU } \\
\text { proL Camr] } \\
\text { LEMO21 (DE3) fhuA2 [lon] ompT gal (IDE3) [dcm] hsdS/ } \\
\text { pLemo(CamR) }\end{array}$ \\
\hline $\mathrm{DH} 5 \alpha$ & $\begin{array}{l}\text { F- } \text { Ф80lacZ } \Delta \text { M15 } \Delta \text { (lacZYA-argF) U169 recA1 endA1 hsdR17 } \\
(\text { (rK-,mK+) phoA supE44 } \lambda \text { - thi-1 gyrA96 relA1 }\end{array}$ \\
\hline JM109 & $\begin{array}{l}\text { endA1 glnV44 thi-1 relA1 gyrA96 recA1 mcrB+ } \Delta \text { (lac-proAB) e14- } \\
{\left[F^{\prime} \text { traD36 proAB + laclq lacZ } \Delta M 15\right] \text { hsdR17(rK- mK+) }}\end{array}$ \\
\hline
\end{tabular}




\subsubsection{Antibodies}

Table 2: Primary antibodies

\begin{tabular}{|c|c|c|c|c|c|c|}
\hline Number & Name & Species & Origin & IF Dilution & $\begin{array}{c}\text { WB } \\
\text { Dilution }\end{array}$ & $\begin{array}{c}\text { PLA } \\
\text { Dilution }\end{array}$ \\
\hline Ab013 & $\alpha-F L A G(M 2)$ & mouse & Sigma & $1: 3,000$ & $1: 1,000$ & \\
\hline Ab029 & a-IPO13 & rabbit & Proteintech & $1: 300$ & $1: 2,000$ & \\
\hline Ab051 & $\alpha-\operatorname{Ran}$ & mouse & $\mathrm{BD}$ & $1: 2,000$ & $1: 5,000$ & \\
\hline Ab121 & $\alpha-F L A G(M 2)$ & rabbit & SIGMA & $1: 1,000$ & $1: 1,000$ & \\
\hline Ab132 & $\alpha-H A$ & mouse & $\begin{array}{l}\text { hybridoma } \\
\text { supernatant }\end{array}$ & $1: 5,000$ & $1: 5,000$ & \\
\hline Ab181 & $\alpha$-Transportin & mouse & $\mathrm{BD}$ & unspecific & $1: 1,000$ & \\
\hline Ab186 & $\alpha-H A$ & mouse & Convance & $1: 1,000$ & $1: 1,000$ & \\
\hline Ab208a & $\alpha-\operatorname{Imp} \beta$ & rabbit & A. Nath & & $1: 1,000$ & \\
\hline Ab219 & $\alpha$-elF1A & rabbit & Abcam & $1: 500$ & $1: 200,000$ & $1: 2,000$ \\
\hline Ab227 & a-IPO13 & rabbit & this work* & $1: 300$ & $1: 1,000$ & $1: 500$ \\
\hline Ab228 & a-IPO13 & rabbit & this work* & $1: 300$ & $1: 1,000$ & \\
\hline Ab234 & a-STRAP & rabbit & Proteintech & - & $1: 300$ & \\
\hline Ab235 & $\alpha-N C A P G$ & rabbit & Proteintech & $1: 100$ & $1: 1,000$ & \\
\hline Ab236 & a-NSUN2 & rabbit & Proteintech & $1: 500$ & $1: 5,000$ & \\
\hline Ab237 & $\alpha-W D R 77$ & rabbit & Proteintech & - & $1: 100$ & \\
\hline Ab238 & a-OLA1 & rabbit & Proteintech & $1: 50$ & $1: 300$ & \\
\hline Ab239 & a-SRP14 & rabbit & Proteintech & $1: 300$ & $1: 1,000$ & \\
\hline Ab240 & a-ERI1 & rabbit & Proteintech & $1: 100$ & $1: 1,000$ & \\
\hline Ab241 & a-EDC3 & rabbit & Proteintech & $1: 50$ & $1: 1,000$ & \\
\hline Ab242 & a-WDR61 & rabbit & Proteintech & $1: 300$ & $1: 1,000$ & \\
\hline Ab243 & $\alpha$-EIF3G & rabbit & Proteintech & $1: 50$ & $1: 100$ & \\
\hline Ab244 & a-APEX & rabbit & Proteintech & $1: 50$ & $1: 500$ & \\
\hline Ab245 & $\alpha-F E N 1$ & rabbit & Proteintech & $1: 500$ & $1: 1,000$ & \\
\hline Ab250 & $\alpha-R T C D 1$ & rabbit & Proteintech & $1: 50$ & $1: 500$ & \\
\hline Ab309 & a-Ubc9 & mouse & Santa Cruz & $1: 100$ & $1: 5,000$ & $1: 500$ \\
\hline
\end{tabular}

Table 3: Secondary antibodies

\begin{tabular}{|c|c|c|c|c|}
\hline Name & Species & Origin & Application & Dilution \\
\hline anti-mouse HRP & goat & Jackson ImmunoResearch & WB & $1: 10,000$ \\
\hline anti-rabbit HRP & goat & Jackson ImmunoResearch & WB & $1: 10,000$ \\
\hline $\begin{array}{l}\text { anti-mouse AlexaFluor }{ }^{\circledR} \\
488 \text { conjugated }\end{array}$ & donkey & Molecular Probes & IF & $1: 1,000$ \\
\hline $\begin{array}{l}\text { anti-mouse AlexaFluor }{ }^{\circledR} \\
594 \text { conjugated }\end{array}$ & donkey & Molecular Probes & IF & $1: 1,000$ \\
\hline $\begin{array}{l}\text { anti-rabbit AlexaFluor }{ }^{\circledR} \\
488 \text { conjugated }\end{array}$ & donkey & Molecular Probes & IF & $1: 1,000$ \\
\hline $\begin{array}{l}\text { anti-rabbit AlexaFluor }{ }^{\circledR} \\
594 \text { conjugated }\end{array}$ & donkey & Molecular Probes & IF & $1: 1,000$ \\
\hline
\end{tabular}




\begin{tabular}{|c|c|c|c|c|}
\hline Name & Species & Origin & Application & Dilution \\
\hline $\begin{array}{l}\text { Duolink® In Situ PLA® } \\
\text { Probe Anti-Rabbit MINUS }\end{array}$ & donkey & Sigma-Aldrich & PLA & $1: 5$ \\
\hline $\begin{array}{l}\text { Duolink® In Situ PLA® } \\
\text { Probe Anti-Mouse PLUS }\end{array}$ & donkey & Sigma-Aldrich & PLA & $1: 5$ \\
\hline FITC-Phalloidin & $\begin{array}{l}\text { Amanita } \\
\text { phalloides }\end{array}$ & Sigma-Aldrich & IF & $1: 600$ \\
\hline
\end{tabular}

\subsubsection{2 siRNAs}

\begin{tabular}{llll}
\hline siRNA & Sequence (5' $\rightarrow$ 3') & Target & Company \\
\hline silPO13_1 & AACAAUAUCAGGAUGAUCCct & IPO13 & Ambion \\
siRNA non- & Silencer Negative Control \#1 siRNA & $\begin{array}{l}\text { scrambled } \\
\text { sequence }\end{array}$ & Ambion \\
targeting $(\mathrm{nt})$ & (Cat. \# AM4635) & & \\
\hline
\end{tabular}

\subsubsection{Oligonucleotides}

Oligonucleotides were obtained from Sigma-Aldrich with a concentration of $100 \mu \mathrm{M}$, a synthesis scale of $0.025 \mu \mathrm{mol}$ and the purification grade desalted. For cloning, restriction sites were added to the 5'-end of the oligonucleotides, as well as, a 5'-end overhang to ensure high cleavage efficiency of the restriction enzymes as specified by the manufacturer (Thermo Scientific). Oligonucleotides were designed to have a melting temperature close to $58^{\circ} \mathrm{C}$ for a three-step PCR protocol or between $69-72^{\circ} \mathrm{C}$ for a twostep protocol using the Phusion DNA polymerase. The melting temperature of oligonucleotides was calculated using the Tm Calculator from Thermo Scientific.

Table 4: Oligonucleotides for cloning

\begin{tabular}{|c|c|c|}
\hline Number & Name & Sequence $\left(5^{\prime} \rightarrow 3^{\prime}\right)$ \\
\hline G1435 & Ubc9_EcoRl_fwd & TTTGAATTCATGTCGGGGATCGCCCTCA \\
\hline G1436 & Ubc9_Sall_rev & AATGTCGACTTATGAGGGCGCAAACTTCTTG \\
\hline G1437 & elF1A_EcoRI_fwd (HPLC) & TTTGAATTCATGCCCAAGAATAAAGGTAAAGG \\
\hline G1438 & elF1A_Sall_rev (HPLC) & TTTGTCGACTTAGATGTCATCAATATCTTCATCATC \\
\hline G1437 & elF1A_EcoRI_fwd (HPLC) & TTTGAATTCATGCCCAAGAATAAAGGTAAAGG \\
\hline G1438 & elF1A_Sall_rev (HPLC) & TTTGTCGACTTAGATGTCATCAATATCTTCATCATC \\
\hline G1448 & elF1A_EcoRI_fwd+spacer+ext & TTTTTTTTTGAATTCTATGCCCAAGAATAAAGGTAAAGG \\
\hline G1449 & elF1A_Sall_rev+ext & TTTTTTTTTGTCGACTTAGATGTCATCAATATCTTCATCATC \\
\hline G1478 & Ubc9_EcoRI_fwd+spacer & TTTTGAATTCTATGTCGGGGATCGCCCTC \\
\hline G1479 & Ubc9_Sall_rev_mouse & AAATGTCGACTTATGAGGGGGCAAACTTCTTCGC \\
\hline G1483 & IP013_Agel_fwd & TTTTACCGGTATGGAGCGGCGGGAGG \\
\hline G1484 & IPO13_Xhol_rev & TTTTCTCGAGTCAGTAGTCAGCTGTGTAATCTGTGCCA \\
\hline G1601 & 01_AIFM2_Kpnl_fwd & TTTTGGTACCATGGGGTCCCAGGTCTCGGT \\
\hline G1602 & 01_AIFM2_BamHI_rev & TTTTGGATCCTTAGGTGGAGACTGCCTCATGGTTTTC \\
\hline
\end{tabular}




\begin{tabular}{|c|c|c|}
\hline Number & Name & Sequence $\left(5^{\prime} \rightarrow 3^{\prime}\right)$ \\
\hline G1603 & 02_PP1B_Kpnl_fwd & TTTTGGTACCATGGCGGACGGGGAGCT \\
\hline G1604 & 02_PP1B_BamHI_rev & TTTTGGATCCTTCCTTTTCTTCGGCGGATTAGCTG \\
\hline G1605 & 03_ZRAB2_Kpnl_fwd & TTTTGGTACCATGTCGACCAAGAATTTCCGAGTCAG \\
\hline G1606 & 03_ZRAB2_BamHI_rev & TTTGGATCCTTGGGTTGTTTAGTGTTTTCACCAATCACCT \\
\hline G1607 & 04_PRMT1_Kpnl_fwd & TTTTGGTACCATGGCGGCAGCCGAGG \\
\hline G1608 & 04_PRMT1_BamHI_rev & TTTTGGATCCTTGCGCATCCGGTAGTCGGTG \\
\hline G1609 & 05_RAE1L_Kpnl_fwd & TTTGGTACCATGAGCCTGTTTGGAACAACCTCAG \\
\hline G1610 & 05_RAE1L_BamHI_rev & TTTGGATCCTTCTTCTTATTCCTGGGCTTTAGCTCTTCG \\
\hline G1611 & 07_CELF1_HindIII_fwd & TTTTAAGCTTTTATGAACGGCACCCTGGACC \\
\hline G1612 & 07_CELF1L_EcoRI_rev & TTTTGAATTCTGTAGGGCTTGCTGTCATTCTTCGA \\
\hline G1613 & 08_HAT1_Kpnl_fwd & TTTTGGTACCATGGCGGGATTTGGTGCTATG \\
\hline G1614 & 08_HAT1_BamHI_rev & TTTGGATCCTTCTCTTGAGCAAGTCGTTCAATAACACG \\
\hline G1615 & 09_IF4A1_HindIII_fwd & TTTTAAGCTTTTATGTCTGCGAGCCAGGATTCC \\
\hline G1616 & 09_IF4A1_EcoRI_rev & TTTTGAATTCTGATGAGGTCAGCAACATTGAGGG \\
\hline G1617 & 10_RUVB1_Kpnl_fwd & TTTGGTACCATGAAGATTGAGGAGGTGAAGAGCACT \\
\hline G1618 & 10_RUVB1_BamHI_rev & TTTGGATCCTTCTTCATGTACTTATCCTGCTGGTCAGCC \\
\hline G1619 & KPNB1_linker_Sacl_fwd & $\begin{array}{l}\text { TTTTGAGCTCTTGGTGGAGGTGGATCTGGAGGTGGAGGTTCT } \\
\text { ATGGAGCTGATCACCATTCTC }\end{array}$ \\
\hline G1620 & TPNO1_linker_Xhol_fwd & $\begin{array}{l}\text { TTTTCTCGAGTTGGTGGAGGTGGATCTGGAGGTGGAGGTTCT } \\
\text { ATGGAGTATGAGTGGAAACCTGACG }\end{array}$ \\
\hline G1621 & IP013_linker_Xhol_fwd & $\begin{array}{l}\text { TTTTCTCGAGTTGGTGGAGGTGGATCTGGAGGTGGAGGTTCT } \\
\text { ATGGAGCGGCGGGAGA }\end{array}$ \\
\hline G1635 & 01_RTCA_Kpnl_fwd & TTTGGTACCATGGCGGGGCCGC \\
\hline G1636 & 01_RTCA_BamHI_rev & TTTGGATCCTTTAGATTTGGATTTGTCATCCCAATTCC \\
\hline G1637 & 02_APEX1_Kpnl_fwd & TTTGGTACCATGCCGAAGCGTGGG \\
\hline G1638 & 02_APEX1_BamHI_rev & TTTGGATCCTTCAGTGCTAGGTATAGGGTGATAGG \\
\hline G1639 & 03_SRP14_Kpnl_fwd & TTTGGTACCATGGTGTTGTTGGAGAGCGAGC \\
\hline G1640 & 03_SRP14_BamHI_rev & TTTGGATCCTTCTGTGCTGCTGTTGCTGCTGT \\
\hline G1641 & 04_NSUN2_EcoRI_fwd & TTTGAATTCAATGGGGCGGCGGTCG \\
\hline G1642 & 04_NSUN2_EcoRI_rev & TTTGAATTCTCCGGGGTGGATGGACCC \\
\hline G1643 & 05_TYW3_Kpnl_fwd & TTTGGTACCATGGATCGCAGCGC \\
\hline G1644 & 05_TYW3_BamHI_rev & TTTGGATCCTTGTAATCTTCAGGGAAGATGGTAAC \\
\hline G1645 & 06_FEN1_HindIII_fwd & TTTTAAGCTTTTATGGGAATTCACGGCCTTGC \\
\hline G1646 & 06_FEN1_Kpnl_rev & TTTGGTACCTTTTTTCCCCTTCGGAACTTCCC \\
\hline G1647 & 07_HNRNPD_Kpnl_fwd & TTTGGTACCATGTCGGAGGAGCAGTTC \\
\hline G1648 & 07_HNRNPD_BamHI_rev & TTTGGATCCTTGTATGGTTTGTAGCTATTTTGATG \\
\hline G1649 & 08_XRCC5_Kpnl_fwd & TTTGGTACCATGGTGCGGTCGGGGA \\
\hline G1650 & 08_XRCC5_BamHI_rev & TTTGGATCCTTTATCATGTCCAATAAATCGTCCACATCA \\
\hline G1651 & 09_XRCC6_BamHI_fwd & TTTGGATCCATGTCAGGGTGGGAGTCATATTAC \\
\hline G1652 & 09_XRCC6_BamHI_rev & TTTGGATCCTTGTCCTGGAAGTGCTTGGTG \\
\hline G1653 & 10_MCM3_HindIII_fwd & TTTTAAGCTTTTATGGCGGGTACCGTGGTG \\
\hline G1654 & 10_MCM3_EcoRI_rev & TTTGAATTCTGATGAGGAAGATGATGCCCTCAGAC \\
\hline G1655 & 11_DBR1_Kpnl_fwd & TTTGGTACCATGCGGGTGGCTGTGGC \\
\hline G1656 & 11_DBR1_BamHI_rev & TTTGGATCCTTAGCTGCATCGTCATCATCATCATC \\
\hline G1657 & 12_SET_Kpnl_fwd & TTTGGTACCATGTCGGCGCCGGC \\
\hline G1658 & 12_SET_BamHI_rev & TTTGGATCCTTGTCATCTTCTCCTTCATCCTCСTCTCC \\
\hline G1659 & 13_EIF2AK2_Kpnl_fwd & TTTGGTACCATGGCTGGTGATCTTTCAG \\
\hline G1660 & 13_EIF2AK2_BamHI_rev & TTTGGATCCTTACATGTGTGTCGTTCATTTTT \\
\hline G1661 & 14_DDX1_HindIII_fwd & TTTAAGCTTTTATGGCGGCCTTCTCC \\
\hline G1662 & 14_DDX1_HindIII_rev & TTTGGATCCTTGAAGGTTCTGAACAGCTGG \\
\hline
\end{tabular}




\begin{tabular}{|c|c|c|}
\hline Number & Name & Sequence $\left(5^{\prime} \rightarrow 3^{\prime}\right)$ \\
\hline G1663 & 15_PRMT5_EcoRI_fwd & TTTGAATTCAATGGCGGCGATGGCG \\
\hline G1664 & 15_PRMT5_EcoRI_rev & TTTGAATTCTGAGGCCAATGGTATATGAGCGGC \\
\hline G1665 & 16_BTF3_Kpnl_fwd & TTTGGTACCATGCGACGGACAGGCGC \\
\hline G1666 & 16_BTF3_BamHI_rev & TTTGGATCCTTGTTTGCCTCATTCTTGGAAGCCTC \\
\hline G1667 & 17_EIF2D_EcoRI_fwd & TTTGAATTCAATGTTTGCCAAGGCCTTTCGG \\
\hline G1668 & 17_EIF2D_EcoRI_rev & TTTGAATTCTCTTCTTCTTGCCAGGTTTGAGGG \\
\hline G1669 & 18_STRAP_Kpnl_fwd & TTTGGTACCATGGCAATGAGACAGACGCCG \\
\hline G1670 & 18_STRAP_BamHI_rev & TTTGGATCCTTGGCCTTAACATCAGGAGCTGAAGG \\
\hline G1673 & 19_TFCP2_Kpnl_fwd & TTTGGTACCATGGCCTGGGCTCTG \\
\hline G1674 & 19_TFCP2_BamHI_rev & TTTGGATCCTTCTTCAGTATGATATGATAGCTATCATTG \\
\hline G1675 & 20_TRIM28_HindIII_fwd & TTTTAAGCTTTTATGGCGGCCTCCGCG \\
\hline G1676 & 20_TRIM28_EcoRI_rev & TTTGAATTCTGGGGCCATCACCAGGGC \\
\hline G1677 & 21_TNIP1_K̄pnl_fwd & TTTGGTACCATGGAAGGGAGAGGACCGTACCG \\
\hline G1678 & 21_TNIP1_BamHI_rev & TTTGGATCCTTCTGAGGCCCCTCACGGTCA \\
\hline G1679 & 22_ERI1_Kpnl_fwd & TTTGGTACCATGGAGGATCCACAGAGTAA \\
\hline G1680 & 22_ERI1_Kpnl_rev & TTTGGTACCAACTTTCTAAAATGTGGCATTTG \\
\hline G1681 & 23_BYSL_HindIII_fwd & TTTTAAGCTTTTATGCCCAAATTCAAGGC \\
\hline G1682 & 23_BYSL_EcoRI_rev & TTTGAATTCTCTCCACGGTGATGGG \\
\hline G1683 & 24_EIF3F_HindIII_fwd & TTTTAAGCTTTTATGGCCACACCGG \\
\hline G1684 & 24_EIF3F_EcoRI_rev & TTTGAATTCTCAGGTTTACAAGTTTTTCATTG \\
\hline G1685 & 25_EIF3G_HindIII_fwd & TTTTAAGCTTTTATGCCTACTGGAGACTTTGA \\
\hline G1686 & 25_EIF3G_EcoRI_rev & TTTGAATTCTGTTGGTGGACGGCTT \\
\hline G1687 & 26_PDCD4_Kpnl_fwd & TTTGGTACCATGGATGTAGAAAATGAGCAG \\
\hline G1688 & 26_PDCD4_BamHI_rev & TTTGGATCCTTGTAGCTCTCTGGTTTAAGACG \\
\hline G1689 & 27_GTF2F2_HindIII_fwd & TTTTAAGCTTTTATGGCCGAGCGC \\
\hline G1690 & 27_GTF2F2_EcoRI_rev & TTTGAATTCTGTCACTCTTTTCTTCTCCTTG \\
\hline G1691 & 28_PPP1CA_Kpnl_fwd & TTTGGTACCATGTCCGACAGCGAGAAGCTCA \\
\hline G1692 & 28_PPP1CA_BamHI_rev & TTTGGATCCTTTTTCTTGGCTTTGGCGGAATTG \\
\hline G1693 & 29_CDK1_HindIII_fwd & TTTTAAGCTTTTATGGAAGATTATACCAAAATAGAG \\
\hline G1694 & 29_CDK1_EcoRI_rev & TTTGAATTCTCATCTTCTTAATCTGATTGTCC \\
\hline G1695 & 30_DDX3_Kpnl_fwd & TTTGGTACCATGAGTCATGTGGCAGTG \\
\hline G1696 & 30_DDX3_BamHI_rev & TTTGGATCCTTGTTACCCCACCAGTCAAC \\
\hline G1697 & 31_XPA_K̄pnl_fwd & TTTGGTACCATGGCGGCGGCCG \\
\hline G1698 & 31_XPA_BamHI_rev & TTTGGATCCTTCATTTTTTCATATGTCAGTTCATGGCCAC \\
\hline G1699 & 32_GPN1_HindIII_fwd & TTTTAAGCTTTTATGCGGTGTCTCTATGGTC \\
\hline G1700 & 32_GPN1_EcoRI_rev & TTTGAATTCTTTTATTGTTTCTCTTCCAGTATTG \\
\hline G1701 & 33_SQSTM1_HindIII_fwd & TTTTAAGCTTTTATGGCGTCGCTCACCGTG \\
\hline G1702 & 33_SQSTM1_EcoRI_rev & TTTGAATTCTCAACGGCGGGGGATGC \\
\hline G1703 & 34_RBM22_Kpnl_fwd & TTTGGTACCATGGCGACCTCTCTGGGTTCC \\
\hline G1704 & 34_RBM22_BamHI_rev & TTTGGATCCTTGGGGCTGCTGTGTTTTCCAG \\
\hline G1705 & 35_NELFA_Kpnl_fwd & TTTGGTACCATGGCGTCCATGCGGG \\
\hline G1706 & 35_NELFA_BamHI_rev & TTTGGATCCTTGGACACATTGGTCATGGGCTTG \\
\hline G1707 & 36_NELFB_Kpnl_fwd & TTTGGTACCATGTTCGCGGGGCTGCA \\
\hline G1708 & 36_NELFB_BamHI_rev & TTTGGATCCTTGAGCGGGGCAGGGGC \\
\hline G1709 & 37_NELFCD_EcoRI_fwd & TTTGAATTCAATGCGCCGCGCTCG \\
\hline G1710 & 37_NELFCD_EcoRI_rev & TTTGAATTCTGTTCACCATGATGAAGTTAGATTTGCAGTG \\
\hline G1739 & tIPÖ13_EcoRI_fwd & TTTGAATTCAATGATCAACAGTGTTCTGCCC \\
\hline G1740 & tIPO13_Xhol_rev & TTTCTCGAGTCAGTAGTCAGCTGTGTAATCTGTG \\
\hline G1765 & 40_OLĀ1_HindIII_fwd & TTTAAGCTTTTATGCCCCCTAAAAAGGGAGGTG \\
\hline
\end{tabular}




\begin{tabular}{lll}
\hline Number & Name & Sequence $\left(\mathbf{5}^{\prime} \rightarrow \mathbf{3}\right.$ ') \\
\hline G1766 & 40_OLA1_EcoRI_rev & TTTGAATTCTTTTCTTCTTCGGTTGTTGAGGTGTG \\
G1767 & 41_WDR77_HindIII_fwd & TTTAAGCTTTTATGCGGAAGGAAACCCCACC \\
G1768 & 41_WDR77_EcoRI_rev & TTTGAATTCTCTCAGTAACACTTGCAGGTCCAGGG \\
G1769 & 38_EDC3_HindIII_fwd & TTTAAGCTTTTATGGCTACAGATTGGCTGGGAAG \\
G1770 & 38_EDC3_EcoRI_rev & TTTGAATTCTAGCAGAGTGCAGTGGGATAACAAACTTG \\
G1771 & 39_WDR61_HindIII_fwd & TTTAAGCTTTTATGACCAACCAGTACGGTATTCTCTTCAAAC \\
G1772 & 39_WDR61_EcoRI_rev & TTTGAATTCTAATTGGACAATCATAGATGTGAATTTCCTGG \\
\hline
\end{tabular}

Table 5: Oligonucleotides for sequencing

\begin{tabular}{lll}
\hline Number & Name & Sequence $\left(\mathbf{5}^{\prime} \rightarrow \mathbf{3}\right.$ ') \\
\hline G828 & Importin13 Bp 1102 for & CAGGATGATATTCTATCC \\
GATC & pcDNA3.1-FP & CTCTGGCTAACTAGAGAAC \\
GATC & pcDNA3.1-RP/1 & CAAACAACAGATGGCTGGC \\
GATC & pEGFP_C2-RP & TTTAAAGCAAGTAAAACCTC \\
GATC & pGEX5-FP & AACGTATTGAAGCTATCCC \\
GATC & SP6 & ATTTAGGTGACACTATAGAA \\
GATC & T3 & ATTAACCCTCACTAAAGGGA \\
GATC & T7 & TAATACGACTCACTATAGGG \\
\hline
\end{tabular}

Table 6: Oligonucleotide for cDNA synthesis

\begin{tabular}{lll}
\hline Name & Sequence $\left(\mathbf{5}^{\prime} \rightarrow \mathbf{3}^{\prime}\right)$ & Origin \\
\hline \multirow{3}{*}{ Oligo(dT) } & TTTTTTTTTTTTTTTTTTTTTTVN & Prof. Markus Bohnsack, \\
& $(\mathrm{V}$ is any nucleotide apart from T; & Department of Molecular \\
& $\mathrm{N}$ is any nucleotide $)$ & Biology, Göttingen \\
\hline
\end{tabular}

\subsubsection{Vectors}

Table 7: Vectors

\begin{tabular}{|c|c|c|c|c|c|}
\hline Number & Name & Tag & Resistance & Application & Origin \\
\hline 4 & pET-28a & $\begin{array}{l}\text { His } \\
\text { (N-terminal) }\end{array}$ & kanamycin & expression & Novagen \\
\hline 30 & pEGFP-C1 & $\begin{array}{l}\text { EGFP } \\
\text { (N-terminal) }\end{array}$ & kanamycin & transfection & Clontech \\
\hline 46 & pGex-6P-1 & $\begin{array}{l}\text { GST } \\
\text { (N-terminal) }\end{array}$ & ampicillin & expression & Amersham \\
\hline 51 & pcDNA 3.1(+) & none & ampicillin & transfection & Invitrogen \\
\hline 52 & pcDNA3.1(+)-HA & $\begin{array}{l}\text { HA-Tag } \\
\text { (C-terminal) }\end{array}$ & ampicillin & transfection & S. Wälde \\
\hline 82 & pGEX-6P-1-MCS2 & $\begin{array}{l}\text { GST } \\
\text { (N-terminal) }\end{array}$ & ampicillin & expression & $\begin{array}{l}\text { this work, MCS from } \\
\# 290 \text { in vector \#46 } \\
(\text { EcoNI, BamHI) }\end{array}$ \\
\hline
\end{tabular}




\subsubsection{Plasmids}

Table 8: Available plasmids

\begin{tabular}{|c|c|c|}
\hline Number & Name & Origin \\
\hline 2 & pET3d-RanQ69L & \\
\hline 186 & pCMV-HA-DDX3 & K.T. Jeang \\
\hline 249 & pCMV-HA-DDX1 & R.J. Pomerantz \\
\hline 290 & pEGFP-GST & D. Doenecke \\
\hline 294 & pdEGFP-C1 & \\
\hline 297 & pdEGFP-GST-NLS (Xenopus) & D. Doenecke \\
\hline 299 & pEYFP-Fos & M. Duterque \\
\hline 300 & pECFP-Jun & M. Duterque \\
\hline 320 & pdGFP-GST-RevNLS & M. Nassiri \\
\hline 328 & pEF-HA-Snurportin1 & S. Hutten \\
\hline 330 & pEF-HA-DDX43 & C. Spillner \\
\hline 333 & pEF-HA-DMAP1 & C. Spillner \\
\hline 335 & pEF-HA-DBC1 & C. Spillner \\
\hline 396 & pdEGFP-Jun & I. Waldmann \\
\hline 403 & pdEGFP-M9 & S. Hutten \\
\hline 467 & pdEGFP-Fos & S. Roloff \\
\hline 510 & pcDNA3.1-HA-Ubc9 & Melchior \\
\hline 545 & pcDNA3.1-TERT-HA & S. Wälde \\
\hline 553 & pcDNA-HA-p53 & EM \\
\hline 651 & pcDNA4-HA-ER alpha & S. Johnson \\
\hline 687 & pCS2plus-FLAG-importin 13 & D. Doenecke \\
\hline 731 & pEGFP-C1-DBC1 & S. Wälde \\
\hline 731 & pEGFP-C1-DBC1 & S. Wälde \\
\hline 755 & pECFP-C1-DDX 43 & S. Wälde \\
\hline 757 & pECFP-C1-DDX 59 & S. Wälde \\
\hline 767 & pmCherry-C1-Sirt1 & S. Wälde \\
\hline 789 & pdEGFP-cNLS-DBC-1 (aa 793-923) & S. Wälde \\
\hline 813 & pCS2plus-FLAG-importin13 (aa 1-410) & D. Doenecke \\
\hline 814 & pCS2plus-FLAG-importin13 (aa 1-669) & D. Doenecke \\
\hline 815 & pCS2plus-FLAG-importin13 (aa 1-784) & D. Doenecke \\
\hline 816 & pCS2plus-FLAG-importin13 (aa 153-963) & D. Doenecke \\
\hline 817 & pCS2plus-FLAG-importin13 (aa 153-784) & D. Doenecke \\
\hline 833 & pEGFP-N1-TERT & C. Frohnert \\
\hline 834 & pEGFP-C1-DMAP & A. Nath \\
\hline 1102 & pEGFP-C1-SQSTM1 & T. Johansen \\
\hline 1112 & GFP-Bystin-like & K. Thakar \\
\hline 1165 & pEGFP-C1-HA-importin 13 (no GFP) & A. Nath \\
\hline 1166 & pEGFP-C1-HA-importin 13-E436R/D481R (no GFP) & A. Nath \\
\hline 1167 & pEGFP-N3-eIF1A & A. Nath \\
\hline 1168 & pEGFP-N3-elF1A-R46E & A. Nath \\
\hline 1239 & pET328-His-zz-tev & F. Vilardi \\
\hline 1511 & pQE80-His-importin 13 & D. Doenecke \\
\hline 1514 & pET23-Ubc9 & R. Geiss-Friedländer \\
\hline 1576 & pET-MCN-GST-importin 13 & F. Bono \\
\hline
\end{tabular}




\begin{tabular}{lll}
\hline Number & Name & Origin \\
\hline 1577 & pcDNA4-SET-myc-His & C. Kaether \\
1689 & pCAGGS-HA-NELFA & Y. Yamaguchi \\
LIFEdb & pEYFP-N-Hsp22 & LIFEdb, DKFZ \\
LIFEdb & pEYFP-N-CPF & LIFEdb, DKFZ \\
LIFEdb & pEYFP-N-Lim6 & LIFEdb, DKFZ \\
LIFEdb & pEYFP-N-Tmp29 & LIFEdb, DKFZ \\
LIFEdb & pEYFP-N-Nip30 & LIFEdb, DKFZ \\
LIFEdb & pEYFP-N-Zinc313 & LIFEdb, DKFZ \\
LIFEdb & pEYFP-N-KIAA1826 & LIFEdb, DKFZ \\
LIFEdb & pEYFP-N-DNAPTP6 & LIFEdb, DKFZ \\
LIFEdb & pEYFP-N-H3.3B & LIFEdb, DKFZ \\
\hline
\end{tabular}

Table 9: Generated plasmids

\begin{tabular}{|c|c|c|c|}
\hline Number & Name & Cloning & Generated by* \\
\hline 1513 & $\begin{array}{l}\text { pCS2plus-FLAG-importin 13- } \\
\text { E436R/D481R }\end{array}$ & $\begin{array}{l}\text { mutant part of IPO13 transferred } \\
\text { from \#1166 to \#687 (Smal/EcoRV) }\end{array}$ & $\begin{array}{l}\text { J. M. Reyes del } \\
\text { Castillo }\end{array}$ \\
\hline 1515 & pGEX-6P-1-Ubc9 & $\begin{array}{l}\text { Ubc9 from \#1514 (G1435, G1436) } \\
\text { in vector \#46 (EcoRI/Sall) }\end{array}$ & \\
\hline 1516 & pGEX-6P-1-eIF1A & $\begin{array}{l}\text { elF1A from } \\
\# 1167 \text { in vector } \# 46 \text { (EcoRI/Sall) }\end{array}$ & \\
\hline 1518 & pET28a-His-elF1A & $\begin{array}{l}\text { elF1A from \#1167 (G1437, G1438) } \\
\text { in vector \#4 (EcoRI/Sall) }\end{array}$ & \\
\hline 1522 & pdEGFP-elF1A & $\begin{array}{l}\text { elF1A from \#1167 (G1448, G1449) } \\
\text { in \# } 294 \text { (EcoRI/Sall) }\end{array}$ & \\
\hline 1547 & pEGFP-GST-Ubc9 & $\begin{array}{l}\text { Ubc9 from \#1514 (G1478, G1479) } \\
\text { in \#290 (EcoRI/Sall) }\end{array}$ & \\
\hline 1569 & pET328-Hzz-importin 13 & $\begin{array}{l}\text { IPO13 from \#1511 (G1483, G1484) } \\
\text { in \#1239 (Agel/Xhol) }\end{array}$ & \\
\hline 1643 & pEGFP-GST-eIF1A & $\begin{array}{l}\text { elF1A from \#1522 in \#290 } \\
\text { (EcoRI/Sall) }\end{array}$ & \\
\hline 1660 & pcDNA3.1-AIFM2-HA & $\begin{array}{l}\text { Insert from cDNA (G1601, G1602) } \\
\text { in vector \#52 (Kpnl/BamHI) }\end{array}$ & C. Spillner \\
\hline 1661 & pEGFP-GST-AIFM2 & $\begin{array}{l}\text { Insert from cDNA (G1601, G1602) } \\
\text { in \#290 (Kpnl/BamHI) }\end{array}$ & C. Spillner \\
\hline 1662 & pcDNA3.1-PPP1CB-HA & $\begin{array}{l}\text { Insert from cDNA (G1603, G1604) } \\
\text { in vector \#52 (Kpnl/BamHI) }\end{array}$ & C. Spillner \\
\hline 1663 & pEGFP-GST-PPP1CB & $\begin{array}{l}\text { Insert from cDNA (G1603, G1604) } \\
\text { in \#290 (Kpnl/BamHI) }\end{array}$ & C. Spillner \\
\hline 1664 & pcDNA3.1-ZRAB2-HA & $\begin{array}{l}\text { Insert from cDNA (G1605, G1606) } \\
\text { in vector \#52 (Kpnl/BamHI) }\end{array}$ & C. Spillner \\
\hline 1665 & pEGFP-GST-ZRAB2 & $\begin{array}{l}\text { Insert from cDNA (G1605, G1606) } \\
\text { in \#290 (Kpnl/BamHI) }\end{array}$ & C. Spillner \\
\hline 1666 & pcDNA3.1-PRMT1-HA & $\begin{array}{l}\text { Insert from cDNA (G1607, G1608) } \\
\text { in vector \#52 (Kpnl/BamHI) }\end{array}$ & C. Spillner \\
\hline 1667 & pEGFP-GST-PRMT1 & $\begin{array}{l}\text { Insert from cDNA (G1607, G1608) } \\
\text { in \#290 (Kpnl/BamHI) }\end{array}$ & C. Spillner \\
\hline
\end{tabular}




\begin{tabular}{|c|c|c|c|}
\hline Number & Name & Cloning & Generated by* \\
\hline 1671 & pcDNA3.1-CELF1-HA & $\begin{array}{l}\text { Insert from cDNA (G1611, G1612) } \\
\text { in vector \#52 (HindIII/EcoRI) }\end{array}$ & C. Spillner \\
\hline 1672 & pEGFP-GST-CELF1 & $\begin{array}{l}\text { Insert from cDNA (G1611, G1612) } \\
\text { in \#290 (HindIII/EcoRI) }\end{array}$ & C. Spillner \\
\hline 1674 & pcDNA3.1-IF4A1-HA & $\begin{array}{l}\text { Insert from cDNA (G1615, G1616) } \\
\text { in vector \#52 (HindIII/EcoRI) }\end{array}$ & C. Spillner \\
\hline 1675 & pEGFP-GST-IF4A1 & $\begin{array}{l}\text { Insert from cDNA (G1615, G1616) } \\
\text { in \#290 (HindIII/EcoRI) }\end{array}$ & C. Spillner \\
\hline 1676 & pcDNA3.1-RUVB1-HA & $\begin{array}{l}\text { Insert from cDNA (G1617, G1618) } \\
\text { in vector \#52 (Kpnl/BamHI) }\end{array}$ & C. Spillner \\
\hline 1677 & pEGFP-GST-RUVB1 & $\begin{array}{l}\text { Insert from cDNA (G1617, G1618) } \\
\text { in \#290 (KpnI/BamHI) }\end{array}$ & C. Spillner \\
\hline 1690 & pcDNA3.1-RTCA-HA & $\begin{array}{l}\text { Insert from cDNA (G1635, G1636) } \\
\text { in vector \#52 (Kpnl/BamHI) }\end{array}$ & \\
\hline 1691 & pcDNA3.1-APEX1-HA & $\begin{array}{l}\text { Insert from cDNA (G1637, G1638) } \\
\text { in vector \#52 (Kpnl/BamHI) }\end{array}$ & \\
\hline 1692 & pcDNA3.1-SRP14-HA & $\begin{array}{l}\text { Insert from cDNA (G1639, G1640) } \\
\text { in vector \#52 (Kpnl/BamHI) }\end{array}$ & \\
\hline 1693 & pcDNA3.1-NSUN2-HA & $\begin{array}{l}\text { Insert from cDNA (G1641, G1642) } \\
\text { in vector \#52 (EcoRI) }\end{array}$ & \\
\hline 1694 & pcDNA3.1-TYW3-HA & $\begin{array}{l}\text { Insert from cDNA (G1643, G1644) } \\
\text { in vector \#52 (KpnI/BamHI) }\end{array}$ & \\
\hline 1695 & pcDNA3.1-HNRNPD-HA & $\begin{array}{l}\text { Insert from cDNA (G1647, G1648) } \\
\text { in vector \#52 (Kpnl/BamHI) }\end{array}$ & \\
\hline 1696 & pcDNA3.1-XRCC6-HA & $\begin{array}{l}\text { Insert from cDNA (G1651, G1652) } \\
\text { in vector \#52 (BamHI) }\end{array}$ & \\
\hline 1697 & pcDNA3.1-MCM3-HA & $\begin{array}{l}\text { Insert from cDNA (G1653, G1654) } \\
\text { in vector \#52 (HindIII/EcoRI) }\end{array}$ & \\
\hline 1698 & pcDNA3.1-DBR1-HA & $\begin{array}{l}\text { Insert from cDNA (G1655, G1656) } \\
\text { in vector \#52 (KpnI/BamHI) }\end{array}$ & \\
\hline 1699 & pcDNA3.1-SET-HA & $\begin{array}{l}\text { Insert from \#1577 (G1657, G1658) } \\
\text { in vector \#52 (Kpnl/BamHI) }\end{array}$ & \\
\hline 1700 & pcDNA3.1-PRMT5-HA & $\begin{array}{l}\text { Insert from cDNA (G1663, G1664) } \\
\text { in vector \#52 (EcoRI) }\end{array}$ & \\
\hline 1701 & pcDNA3.1-BTF3-HA & $\begin{array}{l}\text { Insert from cDNA (G1665, G1666) } \\
\text { in vector \#52 (Kpnl/BamHI) }\end{array}$ & \\
\hline 1702 & pcDNA3.1-STRAP-HA & $\begin{array}{l}\text { Insert from cDNA (G1669, G1670) } \\
\text { in vector \#52 (Kpnl/BamHI) }\end{array}$ & \\
\hline 1703 & pcDNA3.1-TFCP2-HA & $\begin{array}{l}\text { Insert from cDNA (G1673, G1674) } \\
\text { in vector \#52 (Kpnl/BamHI) }\end{array}$ & \\
\hline 1704 & pcDNA3.1-ERI1-HA & $\begin{array}{l}\text { Insert from cDNA (G1679, G1680) } \\
\text { in vector \#52 (Kpnl) }\end{array}$ & \\
\hline 1705 & pcDNA3.1-BYSL-HA & $\begin{array}{l}\text { Insert from \#1112 (G1681, G1682) } \\
\text { in vector \#52 (HindIII/EcoRI) }\end{array}$ & \\
\hline 1706 & pcDNA3.1-EIF3F-HA & $\begin{array}{l}\text { Insert from cDNA (G1683, G1684) } \\
\text { in vector \#52 (HindIII/EcoRI) }\end{array}$ & \\
\hline 1707 & pcDNA3.1-EIF3G-HA & $\begin{array}{l}\text { Insert from cDNA (G1685, G1686) } \\
\text { in vector \#52 (HindIII/EcoRI) }\end{array}$ & \\
\hline 1708 & pcDNA3.1-PPP1CA-HA & Insert from cDNA (G1691, G1692) & \\
\hline
\end{tabular}




\begin{tabular}{|c|c|c|c|}
\hline Number & Name & Cloning & Generated by* \\
\hline & & in vector \#52 (Kpnl/BamHI) & \\
\hline \multirow{2}{*}{1709} & pcDNA3.1-GPN1-HA & Insert from cDNA (G1699, G1700) & \\
\hline & & in vector \#52 (HindIII/EcoRI) & \\
\hline \multirow[t]{2}{*}{1710} & pcDNA3.1-RBM22-HA & Insert from cDNA (G1703, G1704) & \\
\hline & & in vector \#52 (Kpnl/BamHI) & \\
\hline \multirow[t]{2}{*}{1711} & pcDNA3.1-NELFA-HA & Insert from \#1689 (G1705, G1706) & \\
\hline & & in vector \#52 (Kpnl/BamHI) & \\
\hline \multirow[t]{2}{*}{1712} & pcDNA3.1-NELFB-HA & Insert from cDNA (G1707, G1708) & \\
\hline & & in vector \#52 (Kpnl/BamHI) & \\
\hline \multirow[t]{2}{*}{1713} & pcDNA3.1-NELFCD-HA & Insert from cDNA (G1709, G1710) & \\
\hline & & in vector \#52 (EcoRI) & \\
\hline \multirow[t]{2}{*}{1725} & pcDNA3.1-XRCC5-HA & Insert from cDNA (G1649, G1650) & C. Spillner \\
\hline & & in vector \#52 (Kpnl/BamHI) & \\
\hline 1726 & pcDNA3.1-CDK1-HA & Insert from cDNA (G1693, G1694) & C. Spillner \\
\hline \multirow{2}{*}{1727} & pcDNA3.1-EIF2AK2-HA & Insert from cDNA (G1659, G1660) & C. Spillner \\
\hline & & in vector \#52 (Kpnl/BamHI) & \\
\hline 1728 & pcDNA3.1-DDX1-HA & $\begin{array}{l}\text { Insert from \#249 (G1661, G1662) in } \\
\text { vector \#52 (HindIII/EcoRI) }\end{array}$ & C. Spillner \\
\hline 1729 & pcDNA3.1-DDX3-HA & $\begin{array}{l}\text { Insert from \#186 (G1695, G1696) in } \\
\text { vector \#52 (Kpnl/BamHI) }\end{array}$ & C. Spillner \\
\hline \multirow[t]{2}{*}{1730} & pcDNA3.1-SQSTM1-HA & Insert from \#1102 (G1701, G1702) & C. Spillner \\
\hline & & in vector \#52 (HindIII/EcoRI) & \\
\hline \multirow[t]{2}{*}{1731} & pcDNA3.1-XPA-HA & Insert from cDNA (G1697, G1698) & C. Spillner \\
\hline & & in vector \#52 (Kpnl/BamHI) & \\
\hline \multirow[t]{2}{*}{1732} & pEGFP-GST-BTF3 & Insert from \#1701 in \#290 & \\
\hline & & (Kpnl/BamHI) & \\
\hline \multirow[t]{2}{*}{1733} & pEGFP-GST-DBR1 & Insert from \#1698 in \#290 & \\
\hline & & $(\mathrm{Kpnl} / \mathrm{BamHI})$ & \\
\hline 1734 & pEGFP-GST-TFCP2 & $\begin{array}{l}\text { Insert from \#1703 in \#290 } \\
\text { (Knnl/BamHI) }\end{array}$ & \\
\hline \multirow[t]{2}{*}{1735} & pEGFP-GST-XRCC5 & Insert from \#1725 in \#290 & C. Spillner \\
\hline & & $(\mathrm{Kpnl} / \mathrm{BamHI})$ & \\
\hline 1736 & pEGFP-GST-NSUN2 & Insert from \#1693 in \#290 (EcoRI) & C. Spillner \\
\hline \multirow[t]{2}{*}{1737} & pEGFP-GST-DDX1 & Insert from \#1728 in \#290 & C. Spillner \\
\hline & & (HindIII/BamHI) & \\
\hline \multirow[t]{2}{*}{1738} & pEGFP-GST-XPA & Insert from \#1731 in \#290 & C. Spillner \\
\hline & & $(\mathrm{Kpnl} / \mathrm{BamHI})$ & \\
\hline \multirow[t]{2}{*}{1739} & pEGFP-GST-SQSTM1 & Insert from \#1730 in \#290 & C. Spillner \\
\hline & & (HindIII/EcoRI) & \\
\hline \multirow[t]{2}{*}{1740} & pEGFP-GST-EIF2AK2 & Insert from \#1727 in \#290 & C. Spillner \\
\hline & & $(\mathrm{Kpnl} / \mathrm{BamHI})$ & \\
\hline \multirow[t]{2}{*}{1741} & pEGFP-GST-FEN1 & Insert from cDNA (G1645, G1646) & C. Spillner \\
\hline & & in \#290 (HindIII/Kpnl) & \\
\hline \multirow[t]{2}{*}{1745} & pcDNA3.1-EIF2D-HA & Insert from cDNA (G1667, G1668) & \\
\hline & & in vector \#52 (EcoRI) & \\
\hline \multirow[t]{2}{*}{1746} & pcDNA3.1-GTF2F2-HA & Insert from cDNA (G1689, G1690) & \\
\hline & & in vector \#52 (HindIII/EcoRI) & \\
\hline 1747 & pEGFP-GST-RTCA & Insert from \#1690 in \#290 & \\
\hline
\end{tabular}




\begin{tabular}{|c|c|c|c|}
\hline Number & Name & Cloning & Generated by* \\
\hline & & (Kpnl/BamHI) & \\
\hline 1748 & pEGFP-GST-APEX1 & $\begin{array}{l}\text { Insert from \#1691 in \#290 } \\
(\mathrm{Kpnl} / \mathrm{BamHI})\end{array}$ & \\
\hline 1749 & pEGFP-GST-SRP14 & $\begin{array}{l}\text { Insert from \#1692 in \#290 } \\
(\mathrm{Kpnl} / \mathrm{BamHI})\end{array}$ & \\
\hline 1750 & pEGFP-GST-TYW3 & $\begin{array}{l}\text { Insert from \#1694 in \#290 } \\
(\mathrm{Kpnl} / \mathrm{BamHI})\end{array}$ & \\
\hline 1751 & pEGFP-GST-HNRNPD & $\begin{array}{l}\text { Insert from \#1695 in \#290 } \\
(\mathrm{Kpnl} / \mathrm{BamHI})\end{array}$ & \\
\hline 1752 & pEGFP-GST-MCM3 & $\begin{array}{l}\text { Insert from \#1697 in \#290 } \\
\text { (HindIII/EcoRI) }\end{array}$ & \\
\hline 1753 & pEGFP-GST-SET & $\begin{array}{l}\text { Insert from \#1699 in \#290 } \\
(\mathrm{Kpnl} / \mathrm{BamHI})\end{array}$ & \\
\hline 1754 & pEGFP-GST-EIF2D & Insert from \#1745 in \#290 (EcoRI) & \\
\hline 1755 & pEGFP-GST-STRAP & $\begin{array}{l}\text { Insert from \#1702 in \#290 } \\
(\mathrm{Kpnl} / \mathrm{BamHI})\end{array}$ & \\
\hline 1756 & pEGFP-GST-BYSL & $\begin{array}{l}\text { Insert from \#1705 in \#290 } \\
\text { (HindIII/EcoRI) }\end{array}$ & \\
\hline 1757 & pEGFP-GST-EIF3F & $\begin{array}{l}\text { Insert from \#1706 in \#290 } \\
\text { (HindIII/EcoRI) }\end{array}$ & \\
\hline 1758 & pEGFP-GST-EIF3G & $\begin{array}{l}\text { Insert from \#1707 in \#290 } \\
\text { (HindIII/EcoRI) }\end{array}$ & \\
\hline 1759 & pEGFP-GST-PPP1CA & $\begin{array}{l}\text { Insert from \#1708 in \#290 } \\
(\mathrm{Kpnl} / \mathrm{BamHI})\end{array}$ & \\
\hline 1760 & pEGFP-GST-GPN1 & $\begin{array}{l}\text { Insert from \#1709 in \#290 } \\
\text { (HindIII/EcoRI) }\end{array}$ & \\
\hline 1761 & pEGFP-GST-NELFA & $\begin{array}{l}\text { Insert from \#1711 in \#290 } \\
(\mathrm{Kpnl} / \mathrm{BamHI})\end{array}$ & \\
\hline 1762 & pEGFP-GST-NELFB & $\begin{array}{l}\text { Insert from \#1712 in \#290 } \\
(\mathrm{Kpnl} / \mathrm{BamHI})\end{array}$ & \\
\hline 1807 & pEGFP-GST-PRMT5 & Insert from \#1700 in \#290 (EcoRI) & \\
\hline 1808 & pcDNA3.1-PDCD4-HA & $\begin{array}{l}\text { Insert from cDNA (G1687, G1688) } \\
\text { in vector \#52 (Kpnl/BamHI) }\end{array}$ & C. Spillner \\
\hline 1809 & pcDNA3.1-importin 13 & $\begin{array}{l}\text { Insert from \#687 in vector \#52 } \\
\text { (EcoRl/Xhol) }\end{array}$ & C. Spillner \\
\hline 1810 & $\begin{array}{l}\text { pcDNA3.1-importin 13- } \\
\text { E436R/D481R }\end{array}$ & $\begin{array}{l}\text { Insert from \#1513 in vector \#51 } \\
\text { (EcoRI/Xhol) }\end{array}$ & C. Spillner \\
\hline 1812 & $\begin{array}{l}\text { pCS2+MT-FLAG-impotin13 } \\
\text { (aa 526-963) }\end{array}$ & $\begin{array}{l}\text { Insert from \#687 (G1739, G1740) in } \\
\# 687 \text { (EcoRI/Xhol) }\end{array}$ & C. Spillner \\
\hline 1814 & pEGFP-GST-ERI1 & Insert from \#1704 in \#290 (Kpnl) & \\
\hline 1815 & pEGFP-GST-GTF2F2 & $\begin{array}{l}\text { Insert from \#1746 in \#290 } \\
\text { (HindIII/EcoRI) }\end{array}$ & C. Spillner \\
\hline 1827 & pEGFP-GST-NELFCD & Insert from \#1713 in \#290 (EcoRI) & \\
\hline 1829 & pEGFP-GST-XRCC6 & Insert from \#1696 in \#290 (BamHI) & \\
\hline 1843 & pEGFP-GST-PDCD4 & $\begin{array}{l}\text { Insert from \#1808 in \#290 } \\
(\mathrm{Kpnl} / \mathrm{BamHI})\end{array}$ & C. Spillner \\
\hline 1849 & pEGFP-GST-WDR61 & $\begin{array}{l}\text { Insert from \#1868 in \#290 } \\
\text { (HindIII/EcoRI) }\end{array}$ & C. Spillner \\
\hline 1853 & pEGFP-GST-OLA1 & Insert from \#1860 in \#290 & C. Spillner \\
\hline
\end{tabular}




\begin{tabular}{llll}
\hline Number & Name & Cloning & Generated by* \\
\hline 1859 & pCDNA3.1-WDR77-HA & $\begin{array}{l}\text { (HindIII/EcoRI) } \\
\text { Insert from cDNA (G1767, G1768) } \\
\text { in vector \#52 (HindIII/EcoRI) } \\
\text { Insert from cDNA (G1765, G1766) }\end{array}$ & C. Spillner \\
1860 & pCDNA3.1-OLA1-HA & $\begin{array}{l}\text { in vector \#52 (HindIII/EcoRI } \\
\text { Insert from \#1729 in \#290 } \\
\text { (Kpnl/BamHI) }\end{array}$ & C. Spillner \\
1861 & pEGFP-GST-DDX3 & $\begin{array}{l}\text { Insert from \#1859 in \#290 } \\
\text { (HindIII/EcoRI) }\end{array}$ & C. Spillner \\
1862 & pEGFP-GST-WDR77 & $\begin{array}{l}\text { Insert from cDNA (G1771,G1772) } \\
\text { in vector \#52 (HindIII/EcoRI) } \\
\text { Insert from CDNA (G1769, G1770) }\end{array}$ & C. Spillner \\
1868 & pcDNA3.1-WDR61-HA & C. Spillner \\
1871 & pcDNA3.1-EDC3-HA & $\begin{array}{l}\text { Insert from \#1871 in \#290 } \\
\text { (HindIII/EcoRI) }\end{array}$ & C. Spillner \\
\hline
\end{tabular}

*if not indicated otherwise, plasmids were generated by Imke Baade

\subsection{Molecular Biology Methods}

\subsubsection{RNA Isolation from Cellular Extracts}

Total RNA was purified from HeLa P4 cell pellet using the RNeasy Mini Kit (Qiagen), following the protocol 'Purification of Total RNA from Animal Cells using Spin Technology'. RNA was eluted with two times $30 \mu \mathrm{L}$ RNase-free water and stored at $-20^{\circ} \mathrm{C}$. Purity of the RNA was confirmed by measuring the $A_{260} / A_{280}$ ratio with the NanoDrop 2000c. Samples with a ratio of 1.7-1.9 were considered as pure RNA.

\subsection{2 cDNA Synthesis}

For the synthesis of complementary DNA (cDNA) from total RNA, the SuperScript ${ }^{\circledR}$ III reverse transcriptase (Thermo Scientific) was used according to the manufacturer's instructions. Reactions of $13 \mu \mathrm{L}$ were set up with $2 \mu \mathrm{g}$ total RNA, $1 \mu \mathrm{L}$ of $50 \mu \mathrm{M}$ oligo(dT) and $1 \mu \mathrm{L}$ of $10 \mathrm{mM}$ dNTPs in RNase-free water. The reaction mix was incubated at $65^{\circ} \mathrm{C}$ for 5 minutes, followed by incubation on ice for 1 minute. Synthesis of cDNA was initiated by addition of $4 \mu \mathrm{L} 5 x$ First-Strand Buffer, $1 \mu \mathrm{L} 0.1 \mathrm{M}$ DTT, $1 \mu \mathrm{L}$ RiboLockRNase Inhibitor $(40 \mathrm{U} / \mu \mathrm{L})$ as well as $1 \mu \mathrm{L}$ SuperScript ${ }^{\circledR}$ III RT $(200 \mathrm{U} / \mu \mathrm{L})$ and incubation at $50^{\circ} \mathrm{C}$ for 1 hour. Reactions were inactivated at $70^{\circ} \mathrm{C}$ for 15 minutes and cDNA was stored at $-20^{\circ} \mathrm{C}$. 


\subsubsection{Polymerase Chain Reaction (PCR)}

For amplification of specific DNA segments from genomic or plasmid DNA, the polymerase chain reaction (PCR) was used. PCR reactions were performed with Phusion ${ }^{\circledR}$ High-Fidelity DNA Polymerase (Thermo Scientific) according to the manufacturer's recommendations. In brief, $50 \mu \mathrm{L}$ reactions were set up with 1x HF Phusion Buffer, $200 \mu \mathrm{M}$ dNTPs, $5 \mu \mathrm{M}$ forward and reverse primer (see section 2.1.13 for primers), $0.02 \mathrm{U} / \mu \mathrm{L}$ Phusion $^{\circledR}$ High-Fidelity DNA Polymerase and 1 pg -10 ng template DNA. In the case of amplification of a gene from cDNA, 1-2 $\mu \mathrm{L}$ of cDNA was used in the PCR reaction. DNA segments were amplified for 30-35 cycles in a thermocycler using either the 3-step or 2-step (combined annealing and extension step at $72^{\circ} \mathrm{C}$ ) protocol according to the manufacturer's instructions. Annealing temperatures of oligonucleotides were calculated using the 'Tm calculator' from Thermo Scientific (www.thermoscientific.com/pcrwebtools). The extension time was adjusted to the size of the PCR product.

$\mathrm{PCR}$ reactions were analyzed by agarose gel electrophoresis (2.2.4). PCR products were excised from the gel and purified using the NucleoSpin ${ }^{\circledR}$ Gel and PCR Clean-up kit from Macherey-Nagel (2.2.5).

\subsubsection{Agarose Gel Electrophoresis}

DNA fragments were separated according to their size by agarose gel electrophoresis. Depending on the size of the DNA fragments $0.5-2.0 \%$ agarose gels were prepared. The agarose powder was dissolved in 1X TAE buffer by heating in a microwave and regular agitation. After the melted agarose had cooled sufficiently, the SafeView ${ }^{\mathrm{TM}}$ Classic DNA stain was added to a 1:20,000 dilution and the gel was poured. The solidified gel was placed in a running chamber with 1x TAE buffer. Samples prepared with 6x DNA loading buffer together with a molecular weight marker (GeneRuler 100bp DNA Ladder or GeneRuler 1kb DNA Ladder) were loaded onto the gel and separated at $120 \mathrm{~V}$.

Agarose gels were documented with the GeISTICK 'touch' system. DNA fragments used for further cloning were excised from the agarose gel on a UV transilluminator.

\subsubsection{Purification of DNA Fragments from Agarose Gel}

DNA fragments were isolated from agarose gels using the NucleoSpin ${ }^{\circledR}$ Gel and PCR Clean-up kit, following the manufacturer's protocol 'DNA extraction from agarose gels'. DNA fragments were eluted with 25-30 $\mu \mathrm{L}$ elution buffer and the plasmid DNA concentration was determined with the NanoDrop 2000c. 


\subsubsection{DNA Restriction Digestion}

DNA was digested at $37^{\circ} \mathrm{C}$ for $1-2 \mathrm{~h}$ using the restriction enzyme ratio and buffer recommended by the 'DoubleDigest Calculator' tool from Thermo Scientific (www.thermoscientific.com/doubledigest). For preparative digestion, $25 \mu \mathrm{L}$ of the PCR product and 2-5 $\mu \mathrm{g}$ plasmid DNA was cut with not more than $1 \mu \mathrm{L}$ of enzyme $(10 \mathrm{U} / \mu \mathrm{L})$ per one $\mu \mathrm{g}$ of DNA in a 20-50 $\mu \mathrm{L}$ reaction volume. For restriction analysis of plasmid DNA from small scale DNA preparations, $5 \mu \mathrm{L}$ of the eluted plasmid DNA was digested with not more than $0.2 \mu \mathrm{L}$ of enzyme $(10 \mathrm{U} / \mu \mathrm{L})$ in a $10 \mu \mathrm{L}$ reaction volume. Alternatively, digestion was also performed with FastDigest enzymes from Thermo Scientific following the manufacturer's instructions.

Digested DNA was analyzed by agarose gel electrophoresis (2.2.4).

\subsubsection{Dephosphorylation of Linearized Vectors}

Cut vector DNA was dephosphorylated with FastAP Thermosensitive Alkaline Phosphatase (Thermo Scientific) to remove the 5' phosphate from the DNA to prevent recircularization of the vector. The digested vector was incubated with $1 \cup$ Alkaline Phosphatase per $1 \mu \mathrm{g}$ DNA in $1 \times$ FastAP reaction buffer for 10 minutes at room temperature. The enzyme was inactivated for 5 minutes at $75^{\circ} \mathrm{C}$.

\subsubsection{Ligation of DNA Fragments}

DNA fragments were ligated into linearized vectors using the T4 DNA ligase (Thermo Scientific). Ligation was performed with $100 \mathrm{ng}$ linear vector DNA, a 3:1 molar ratio of insert DNA to vector DNA, 1x T4 DNA ligase buffer and $1 \mathrm{U}$ of T4 DNA ligase in a total reaction volume of $10 \mu \mathrm{L}$. The reaction mix was incubated for 1 hour at room temperature and transformed into Escherichia coli (E. coli) $\mathrm{DH} 5 \mathrm{a}(2.2 .9)$.

\subsubsection{Transformation of E. coli with Plasmid DNA}

For transformation, $10 \mu \mathrm{L}$ of ligation mixture or $0.1 \mu \mathrm{g}$ of plasmid-DNA was added to $50 \mu \mathrm{L}$ of chemically competent cells. E. coli DH5a cells were used for cloning. The transformation mix was incubated on ice for 15 minutes, before the cells were heat shocked for 90 seconds at $42^{\circ} \mathrm{C}$. The cells were recovered on ice for 2 minutes, resuspended in $300 \mu \mathrm{L}$ SOC-medium and incubated at $37^{\circ} \mathrm{C}$ for 1 hour at $850 \mathrm{rpm}$. Half of the transformation mix was spread on a selective LB agar plate and incubated overnight at $37^{\circ} \mathrm{C}$. 


\subsubsection{Small Scale Plasmid DNA Isolation}

Plasmid DNA was isolated from 2-4 mL E. coli overnight culture using the NuceloSpin ${ }^{\circledR}$ Plasmid kit following the 'Isolation of high-copy plasmid DNA from E.coli' protocol. For the overnight culture, 3-5 mL of LB medium containing the respective antibiotics were inoculated with a single colony picked from a selective LB agar plate. The culture was incubated overnight at $37^{\circ} \mathrm{C}, 180 \mathrm{rpm}$. The purified DNA was eluted with $50 \mu \mathrm{L}$ elution buffer. The correctness of the plasmid DNA was confirmed by restriction analysis and sequencing.

\subsubsection{Large Scale Plasmid DNA Isolation}

For large scale preparation of plasmid DNA, the NucleoBond ${ }^{\mathrm{TM}}$ Xtra Midi kit was used following the supplied protocols 'High-copy plasmid purification' or 'Low-copy plasmid purification'. For the overnight culture, $100-200 \mathrm{~mL}$ of LB medium supplemented with the appropriate antibiotics was inoculated with a single colony. The culture was incubated overnight at $37^{\circ} \mathrm{C}, 180 \mathrm{rpm}$. The plasmid DNA was eluted with desalted water and the concentration was adjusted to $1 \mathrm{mg} / \mathrm{mL}$ using the Nanodrop 2000c.

\subsubsection{Sequencing}

To verify the success of ligation and ascertain that no frame-shifts or mutations were introduced, the isolated ligation product was sent for sequencing to the company GATC Biotech. Sequencing primers were selected from the GATC PrimerScout tool (www.gatc-biotech.com) or custom-made primers were used (2.1.13).

\subsection{Biochemical Methods}

\subsubsection{SDS-PAGE}

To separate proteins according to their electrophoretic mobility an SDS-PAGE was performed. Depending on the size of the target protein SDS gels with separation gels of 6-15\% acrylamide were prepared and overlaid with a 4\% stacking gel. Protein samples or cell lysates were mixed with reducing SDS sample buffer and heated for 5-10 minutes at $95^{\circ} \mathrm{C}$. The prepared samples, as well as a protein ladder (PageRuler Prestained, PageRuler Unstained), were loaded onto the polyacrylamide gel. Gel electrophoresis was performed with 1x Laemmli buffer in Mighty Small II Mini Vertical Electrophoresis Units 
(Hoefer) with 25-30 mA per gel until the bromophenol blue dye had completely passed through the gel.

Proteins were either visualized by Coomassie staining (2.3.2), silver staining (2.3.3) or by targeting the protein of interest with a specific antibody in a Western blot (2.3.4).

Additionally to the self-made gels, two types of precast gradient gels were used. The 4-20\% Mini-PROTEAN ${ }^{\mathrm{R}}$ TGXTM $^{\mathrm{TM}}$ Precast Protein Gels (BioRad) were run with 1x Laemmli buffer in SE250 Mighty Small II Mini Vertical Electrophoresis Units (Hoefer) at $100 \mathrm{~V}$ until the samples had passed through the separation gel and then at $300 \mathrm{~V}$ for $\sim 30$ minutes. The NuPAGE ${ }^{R}$ Novex $^{R}$ 4-12\% Bis-Tris gradient gels (Invitrogen) were run with $1 \times$ NUPAGE $^{R}$ MES SDS running buffer in XCell SureLock ${ }^{R}$ Mini-Cells at $200 \mathrm{~V}$ for $\sim 45$ minutes.

\subsubsection{Coomassie Staining}

Polyacrylamide gels were rinsed with water, fixed for 10 minutes to one hour with Coomassie fixation solution and stained for one hour to overnight in Coomassie staining solution on a shaker. Gels were destained with deionized water, or if the background signal was too high, with Coomassie destaining solution. Coomassie-stained gels were documented with the LAS-3000 and imported into the Fiji software for editing and quantification.

\subsubsection{Silver Staining}

Compared to Coomassie staining of polyacrylamide gels, silver staining is more sensitive and allows detection of low amounts of proteins. Silver staining was performed with the Pierce ${ }^{\circledR}$ Silver Stain kit following the manufacturer's instructions. Silver-stained gels were documented with the LAS-3000 and edited with Fiji software.

\subsubsection{Western Blotting}

For the immunological detection of specific proteins, Western Blots were performed. Proteins separated by SDS-PAGE were transferred to a Amersham Protran $0.45 \mu \mathrm{m}$ NC Nitrocellulose Blotting Membrane (GE Healthcare) using a Mini Trans-Blot ${ }^{\circledR}$ Cell (Bio-Rad). The transfer was performed in Western blot transfer buffer (25 mM Tris, $193 \mathrm{mM}$ glycine, $0.02 \%$ SDS, 20\% methanol) at $100 \mathrm{~V}$ and $350 \mathrm{~mA}$. Transfer times were adjusted depending on the protein size and percentage of the resolving gel.

The 4-20\% Mini-PROTEAN ${ }^{\mathrm{R}}$ TGXTM Precast Protein Gels from BioRad were pre-incubated for 30 minutes in $1 x$ Western blot transfer buffer prior to transfer. The 
NuPAGE $^{R}$ Novex $^{R} 4-12 \%$ Bis-Tris gradient gels from Invitrogen were blotted at $60 \mathrm{~V}$ for 90 minutes.

The quality of protein transfer was tested by staining the membrane with PonceauS staining solution for 2 minutes and visualization of the protein bands by rinsing several times with $0.1 \%$ acetic acid. Stained membranes were documented with the LAS-3000 (Fujifilm) and destained with PBS-T (137 mM NaCl, $2.7 \mathrm{mM} \mathrm{KCl,} 10 \mathrm{mM} \mathrm{Na}{ }_{2} \mathrm{HPO}_{4}, 1.8$ $\mathrm{mM} \mathrm{KH}_{2} \mathrm{PO}_{4}, 0.1 \%$ Tween-20) for further processing.

The Nitrocellulose membrane was blocked with 4\% milk powder in PBS-T for 45 minutes at room temperature to prevent unspecific binding of antibodies, followed by incubation with the primary antibody (2.1.11) diluted in $4 \%$ milk powder/PBS-T overnight at $4^{\circ} \mathrm{C}$. The membrane was washed three times with PBS-T for 10 minutes and incubated with the secondary HRP-coupled antibody (2.1.11) diluted 1:10,000 in 4\% milk powder/PBS-T for 1 hour at room temperature. Unbound antibody was removed by washing three times for 10 minutes with PBS-T and bound antibodies were detected by chemiluminescence using the Immobilon ${ }^{\mathrm{TM}}$ Western Chemiluminescent HRP Substrate (Millipore) kit and exposure to Amersham Hyperfilm ${ }^{\mathrm{TM}}$ ECL (GE Healthcare) or Medix XBU medical x-ray films (FOMA Bohemia). Film exposure times depended on the strength of the signal and exposed films were developed with the developer machine CURIX60 (Agfa).

\subsubsection{Protein Purification}

$\underline{\text { His-importin } 13}$

His-importin 13 was purified as described before (Mingot et al., 2001). For protein expression, the plasmid coding for His-importin 13 was transformed (2.2.9) into JM109 E. coli bacteria and plated on an LB agar plate supplemented with ampicillin. The next day several colonies were picked and used for inoculation of $100 \mathrm{~mL} 2 \mathrm{x}$ YT-medium supplemented with $2 \%$ glycerol, $30 \mathrm{mM} \mathrm{K}_{2} \mathrm{HPO}_{4}$ and $100 \mu \mathrm{g} / \mathrm{mL}$ ampicillin. The $100 \mathrm{~mL}$ starter culture was grown overnight at $37^{\circ} \mathrm{C}, 150 \mathrm{rpm}$ and was used to inoculate $700 \mathrm{~mL}$ of 2x YT-medium supplemented with 2\% glycerol, $30 \mathrm{mM} \mathrm{K}_{2} \mathrm{HPO}_{4}$ and $100 \mu \mathrm{g} / \mathrm{mL}$ ampicillin. The culture was grown at $37^{\circ} \mathrm{C}, 110 \mathrm{rpm}$ to an $\mathrm{OD}_{600}$ of 0.6 before $1.2 \mathrm{~L}$ of cold 2x YT-medium supplemented with $2 \%$ glycerol, $30 \mathrm{mM} \mathrm{K}_{2} \mathrm{HPO}_{4}$ and $100 \mu \mathrm{g} / \mathrm{mL}$ ampicillin were added. The temperature was shifted to $16^{\circ} \mathrm{C}$ and the culture was grown to an $\mathrm{OD}_{600}$ of 0.75 prior to induction of protein expression with $0.1 \mathrm{mM}$ IPTG overnight at $16^{\circ} \mathrm{C}$. Bacterial cells were collected by centrifugation for 20 minutes at 4,200 rpm (Centrifuge J6-MI with rotor JS 4.2, Beckman Coulter), washed with PBS and stored at $-80^{\circ} \mathrm{C}$ for protein purification. 
Each bacterial pellet from $1.5 \mathrm{~L}$ of culture was resuspended in $35 \mathrm{~mL}$ His buffer $(50 \mathrm{mM}$ Tris $\mathrm{pH} 7.5,500 \mathrm{mM} \mathrm{NaCl}, 10 \mathrm{mM} \mathrm{MgAc}, 5 \%$ glycerol, $10 \mathrm{mM} \beta$-mercaptoethanol, $0.1 \mathrm{mM}$ PMSF, $1 \mu \mathrm{g} / \mathrm{mL}$ of each AP and LP) and disrupted with three to four cycles using an EmulsiFlex-C3 (Avestin). Lysates were cleared by centrifugation at $100,000 \times \mathrm{g}, 4^{\circ} \mathrm{C}$ for 30 minutes. The supernatant was collected and incubated with $0.25 \mathrm{~mL}$ equilibrated Ni-NTA Sepharose and $10 \mathrm{mM}$ imidazole rotating for 1 hour at $4^{\circ} \mathrm{C}$. The Ni-beads were washed two times with His buffer containing $10 \mathrm{mM}$ imidazole and once with $20 \mathrm{mM}$ imidazole. Beads were transferred to an empty column and proteins were eluted with His buffer containing $300 \mathrm{mM}$ imidazole. The eluate was filtered with a Minisart RC 15 single use syringe filter and further purified over a HiLoad 26/60 Superdex 200 prep grade column connected to a ÄKTApurifier system (Amersham Biosciences) using pulldown buffer (50 mM Tris pH 7.4, $200 \mathrm{mM} \mathrm{NaCl}, 5 \%$ glycerol, $2 \mathrm{mM}$ DTT). Peak fractions containing the protein of interest were pooled and concentrated using a Spin-X UF concentrator (His-importin 13: MWCO=50 kDa; His-elF1A: MWCO=10kDa). The protein was aliquoted, flash frozen and stored at $-80^{\circ} \mathrm{C}$.

\section{Other His-tagged proteins}

All His-tagged proteins were purified using the same protocol as for His-importin 13 but different expression strains and expression conditions were used. Plasmids coding for Hzz-importin 13 (contains His- and zz-tag) and the Hzz-tag were transformed into BL21 CodonPlus (DE3) and plasmids coding for His-elF1A were transformed into BL21 (DE3) E. coli bacteria.

For protein expression, $200 \mathrm{~mL}$ LB medium was inoculated with several colonies from a selective LB agar plate and incubated overnight at $37^{\circ} \mathrm{C}, 150 \mathrm{rpm}$. For large scale protein expression, $1.5 \mathrm{~L}$ of LB medium was inoculated 1:100 with the overnight culture and grown at $37^{\circ} \mathrm{C}, 110 \mathrm{rpm}$ to an $\mathrm{OD}_{600}$ of $0.5-0.8$. Protein expression was induced with $0.1 \mathrm{mM}$ Isopropyl $\beta$-D-1-thiogalactopyranoside (IPTG) for His-importin 13 and 1 mM IPTG for His-elF1A and cultures were grown overnight at $16^{\circ} \mathrm{C}, 110 \mathrm{rpm}$. Bacterial cells were harvested by centrifugation for 20 minutes at 4,200 rpm (Centrifuge J6-MI with rotor JS 4.2, Beckman Coulter) and washed once with PBS. Cell pellets were either flash frozen and stored at $-80^{\circ} \mathrm{C}$ or were used directly for protein purification. The His-tagged proteins were purified as described for His-importin 13. 


\section{GST-tagged proteins}

Plasmids coding for GST-tagged proteins were transformed into BL21 CodonPlus (DE3), grown overnight at $37^{\circ} \mathrm{C}$ and several colonies were used for inoculation of $200 \mathrm{~mL}$ LB-medium supplemented with $100 \mu \mathrm{g} / \mathrm{mL}$ ampicillin. Overnight cultures grown at $37^{\circ} \mathrm{C}$, $150 \mathrm{rpm}$ were used to inoculate $1.5 \mathrm{~L}$ cultures to a 1:100 dilution. Cultures were grown at $37^{\circ} \mathrm{C}, 110 \mathrm{rpm}$ to an $\mathrm{OD} 600=0.5-0.8$ before protein expression was induced with $1 \mathrm{mM}$ IPTG. Protein expression was allowed to occur overnight at $16^{\circ} \mathrm{C}, 110 \mathrm{rpm}$. Cells were harvested at 4,200 rpm (Centrifuge J6-MI with rotor JS 4.2, Beckman Coulter) for 20 minutes and either flash frozen and stored at $-80^{\circ} \mathrm{C}$ or were directly used for protein purification.

Cell pellets were resuspended in $35 \mathrm{~mL}$ GST buffer (50 mM Tris pH 6.8, $300 \mathrm{mM} \mathrm{NaCl}$, $1 \mathrm{mM} \mathrm{MgCl} 2,2 \mathrm{mM}$ DTT, $0.1 \mathrm{mM}$ PMSF, $1 \mu \mathrm{g} / \mathrm{mL}$ of each AP and LP) and disrupted with three to four cycles using an EmulsiFlex-C3 (Avestin). Lysates were cleared by centrifugation at $100,000 \times \mathrm{g}, 4^{\circ} \mathrm{C}$ for 30 minutes and supernatants were transferred to $0.25 \mathrm{~mL}$ GST beads equilibrated in GST-buffer. After incubation for 1 hour with rotation at $4^{\circ} \mathrm{C}$, beads were washed three times with GST buffer and transferred to an empty column. Bound protein was eluted with GST-buffer containing $15 \mathrm{mM}$ reduced glutathione, $\mathrm{pH} 8$. GST-importin 13 was filtered with a Minisart RC 15 single use syringe filter and further purified over a HiLoad 26/60 Superdex 200 prep grade column attached to a ÄKTApurifier system (Amersham Biosciences) using pulldown buffer (50 mM Tris pH 7.4, $200 \mathrm{mM} \mathrm{NaCl}$, $5 \%$ glycerol, $2 \mathrm{mM}$ DTT) and concentrated with a Spin-X UF concentrator (MWCO $=50 \mathrm{kDa}$ ). GST-elF1A and GST-Ubc9 were dialyzed against pulldown buffer using a dialysis membrane with a molecular weight cut-off of 12-14 kDa. To remove any residual glutathione, the buffer was changed after 1 hour, overnight and then again after 1 hour. Proteins were aliquoted, flash frozen and stored at $-80^{\circ} \mathrm{C}$.

\section{$\underline{\text { Ubc9 (no tag) }}$}

Ubc9 without a tag was purified as described in Pichler et al., 2002 (177). Several colonies from a selective LB agar plate transformed with plasmid DNA coding for Ubc9 were used for inoculation of $200 \mathrm{~mL}$ LB medium supplemented with $100 \mu \mathrm{g} / \mathrm{mL}$ ampicillin. The culture was grown overnight at $37^{\circ} \mathrm{C}, 110 \mathrm{rpm}$ and used for inoculation of a $1.5 \mathrm{~L}$ culture to a 1:100 dilution. Cells were grown at $37^{\circ} \mathrm{C}, 110 \mathrm{rpm}$ to an $\mathrm{OD}_{600}=0.5-0.6$ before protein expression was induced with $1 \mathrm{mM}$ IPTG for 4 hours at $37^{\circ} \mathrm{C}, 110 \mathrm{rpm}$. Cells were harvested at 4,200 rpm (Centrifuge J6-MI with rotor JS 4.2, Beckman Coulter), resuspended in $60 \mathrm{~mL}$ Ubc9 buffer $(50 \mathrm{mM} \mathrm{Na-phosphate} \mathrm{pH}$ 6.5, $50 \mathrm{mM} \mathrm{NaCl}, 0.1 \mathrm{mM}$ 
PMSF, $1 \mu \mathrm{g} / \mathrm{mL}$ of each AP and LP, $1 \mathrm{mM} \mathrm{DTT}$ ), flash frozen and stored at $-80^{\circ} \mathrm{C}$ at least until the next day. Freezing of bacterial cells at $-80^{\circ} \mathrm{C}$ overnight and rapid thawing was sufficient for cell lysis and release of Ubc9.

Cell lysates were thawed, supplemented with $0.1 \mathrm{mM} \mathrm{PMSF}, 1 \mu \mathrm{g} / \mathrm{mL}$ of each AP and LP and $1 \mathrm{mM}$ DTT and cleared by centrifugation at $100,000 \times \mathrm{g}, 4^{\circ} \mathrm{C}$ for 30 minutes. Cleared supernatants were run over $7.5 \mathrm{~mL}$ of SP-Sepharose (Roth) equilibrated in Ubc9 buffer. The flow-through was discarded and the SP-Sepharose was washed with two column volumes of Ubc9 buffer. Bound Ubc9 was eluted with 2-3 column volumes of Ubc9 buffer containing $300 \mathrm{mM} \mathrm{NaCl}$ and protein containing fractions were pooled, concentrated with a Spin-X UF concentrator (MWCO=5 kDa), filtered with a Minisart RC 15 single use syringe filter and further purified over a HiLoad 26/60 Superdex 75 prep grade column connected to a ÄKTApurifier system (Amersham Biosciences) using transport buffer (20 mM HEPES, $110 \mathrm{mM}$ KOAc, $2 \mathrm{mM} \mathrm{Mg(OAc)}$, 1 mM EGTA, pH 7.3, 2 mM DTT). Peak fractions containing Ubc9 were pooled, concentrated with a Spin-X UF concentrator $(\mathrm{MWCO}=5 \mathrm{kDa})$, aliquoted, flash frozen and stored at $-80^{\circ} \mathrm{C}$.

\section{RanQ69L}

RanQ69L was expressed and purified as described in Melchior et al., 1995 (178). For the SILAC experiments, a RanQ69L protein stock prepared by Christiane Spillner that was purified over DEAE-Sepharose and a HiLoad 26/60 Superdex 75 prep grade column was used. For all other experiments, a self-made RanQ69L protein stock was used that was also purified over DEAE-Sepharose and a HiLoad 26/60 Superdex 75 prep grade column but additionally was further purified over a MonoS 5/50 GL column.

\subsubsection{Protein Concentration Determination by Densitometry}

To determine the protein concentration by densitometry, 0.5, 1.0 and $1.5 \mu \mathrm{g}$ BSA as well as 1,2 and $3 \mu \mathrm{L}$ of the protein sample were applied on a polyacrylamide gel, separated by SDS-PAGE (2.3.1), Coomassie stained (2.3.2) and imaged using the LAS-3000. Protein concentrations were determined with Fiji using BSA as a standard protein to generate a standard curve.

\subsubsection{Loading of RanQ69L with GTP}

The RanQ69L mutant, which can bind but not hydrolyze GTP was loaded with GTP following a protocol adapted from Kehlenbach et al., 1999 (58). Purified RanQ69L was incubated with $2.5 \mathrm{mM}$ GTP and $15 \mathrm{mM}$ EDTA in transport buffer for 30 minutes at room 
temperature. The loading reaction was stopped by the addition of $40 \mathrm{mM} \mathrm{MgCl}$ for 15 minutes on ice. GTP-loaded RanQ69L was aliquoted, snap frozen and stored at $-80^{\circ} \mathrm{C}$.

\subsubsection{Binding Assays}

\section{Binding Assay with Purified Proteins}

To analyze the interaction between importin 13 and potential substrates, binding assays were performed. For this, either GST-tagged proteins were immobilized to Glutathione Sepharose High-Performance beads (GE Healthcare) or Hzz-tagged proteins were immobilized to IgG-Sepharose 6 Fast Flow (GE Healthcare). Beads (20 $\mu \mathrm{L}$ ) were incubated with $5 \mathrm{mg} / \mathrm{mL}$ BSA in PBS $\left(137 \mathrm{mM} \mathrm{NaCl}, 2.7 \mathrm{mM} \mathrm{KCl}, 10 \mathrm{mM} \mathrm{Na}{ }_{2} \mathrm{HPO}_{4}\right.$, $\left.1.8 \mathrm{mM} \mathrm{KH}_{2} \mathrm{PO}_{4}, \mathrm{pH} 7.5\right)$ as a blocking reagent with rotation for 30 minutes at $4^{\circ} \mathrm{C}$, followed by immobilization of 5-20 $\mu \mathrm{g}$ GST- or Hzz-bound fusion proteins rotating for one hour at $4^{\circ} \mathrm{C}$ in the same buffer. The beads were washed three times with $1 \mathrm{~mL} P B S$ containing $2 \mathrm{mg} / \mathrm{mL}$ BSA and incubated with an equimolar or excess amount of the protein of interest in a total volume of $500 \mu \mathrm{L}$, rotating for one hour at $4^{\circ} \mathrm{C}$. Unbound proteins were removed by washing three times with $1 \mathrm{~mL}$ PBS. Proteins bound to Glutathione Sepharose were eluted with $4 \times$ SDS sample buffer and proteins bound to IgG-Sepharose were eluted with either glycine or magnesium chloride. Glycine elution was done with $40 \mu \mathrm{L} 0.1 \mathrm{M}$ glycine, pH 3 for 1 minute with constant mixing, followed by neutralization with $5 \mu \mathrm{L} 1 \mathrm{M}$ Tris, $\mathrm{pH}$ 10.4. The beads were pellet by centrifugation and the supernatant was collected and mixed 1:1 with 4x SDS sample buffer. Magnesium chloride elution was done with $150 \mu \mathrm{L} 1.5 \mathrm{M} \mathrm{MgCl}_{2}$ in $50 \mathrm{mM}$ Tris, $\mathrm{pH} 7.4$, vortexing and incubation for 10 minutes on ice. The supernatant was collected and precipitated with 1,350 $\mu \mathrm{L} 100 \%$ isopropanol, vortexing and incubation for 10 minutes on ice, followed by centrifugation for 20 minutes at $14,000 \times \mathrm{g}$. The supernatant was carefully removed and the protein pellet air evaporated prior to reconstitution of the pellet in $60 \mu \mathrm{L} 4 \mathrm{x}$ SDS-sample buffer. To analyze the efficiency of the first protein elution, a second elution step was performed by washing the beads two times with transport buffer and eluting with $60 \mu \mathrm{L} 4 \mathrm{x}$ SDS-sample buffer. All eluates were boiled for 10 minutes at $95^{\circ} \mathrm{C}$ and analyzed by SDS-PAGE (2.3.1) and optionally Western blotting (2.3.4).

\section{Binding Assay with Cell Lysate}

The binding assay with cell lysate was performed similarly to the binding assay with recombinantly purified proteins. The only differences were that transport buffer $(20 \mathrm{mM}$ HEPES, $110 \mathrm{mM}$ KOAc, $2 \mathrm{mM} \mathrm{Mg(OAc)})_{2}, 1 \mathrm{mM}$ EGTA, pH 7.3, $2 \mathrm{mM}$ DTT, $1 \mu \mathrm{g} / \mathrm{ml}$ of 
each AP and LP and 1x Roche complete protease inhibitor cocktail) instead of PBS was used as a buffer and that as a source of the protein of interest a cell lysate was used instead of a purified protein. The cells lysate was prepared by trypsinizing two HeLa P4 $15-\mathrm{cm}$ plates and collecting the cells in transport buffer containing $10 \%$ fetal calf serum. The fetal calf serum was removed by washing three times with transport buffer and the cells were adjusted to a concentration of $1 \times 10^{8}$ cells $/ \mathrm{mL}$. Cells were permeabilized with three freeze/thaw cycles in liquid nitrogen or with $0.07 \%$ digitonin on ice for $3-5$ minutes. Digitonin permeabilization efficiency was checked under the microscope by mixing $1 \mu \mathrm{L}$ of cell suspension with Trypan blue. The lysate was cleared by centrifugation at $1,500 \times \mathrm{g}$ for 15 minutes, $4^{\circ} \mathrm{C}$ to remove nuclei and cell debris, followed by ultracentrifugation at $100,000 \times \mathrm{g}$ for 30 minutes, $4^{\circ} \mathrm{C}$. The supernatant was collected and added to the prepared affinity matrix as described above.

\subsubsection{Antibody Purification}

For the production of rabbit polyclonal antibodies against importin 13, two rabbits were immunized with His-importin 13 (2.3.5) by the company Seramun Diagnostica GmbH. To allow for the purification of anti-importin 13 antibodies from the raised hyperimmune serum, an affinity matrix was generated. For this, $0.5 \mathrm{mg}$ GST-importin 13 was dialyzed overnight against carbonate buffer (0.2 $\left.\mathrm{M} \mathrm{NaHCO}_{3} / \mathrm{Na}_{2} \mathrm{CO}_{3}, \mathrm{pH} 8.9\right)$ at $4^{\circ} \mathrm{C}$ with three buffer changes, after minimum one hour, overnight and one hour to remove any free amine groups. The dialyzed GST-importin 13 was immobilized to $0.8 \mathrm{~g}$ Cyanogen bromide-activated Sepharose 4B (swelled in $1 \mathrm{mM}$ cold $\mathrm{HCl}$ for 30 minutes, followed by two washes with deionized water and one wash with $0.2 \mathrm{M} \mathrm{NaHCO} / \mathrm{Na}_{2} \mathrm{CO}_{3}, \mathrm{pH}$ 8.9) for one hour at room temperature, followed by overnight incubation at $4^{\circ} \mathrm{C}$. The affinity matrix was washed two times with carbonate buffer and remaining coupling sites were blocked with $100 \mathrm{mM}$ ethanolamine in carbonate buffer for one hour at room temperature. After three more washes with carbonate buffer, the affinity matrix was equilibrated with PBS containing $500 \mathrm{mM} \mathrm{NaCl}$, split into two parts and incubated with half of the serum from each rabbit (rabbit $83 / 14=12.5 \mathrm{~mL}$ and rabbit $85 / 14=11.5 \mathrm{~mL}$ ) at $4^{\circ} \mathrm{C}$, overnight and slow rotation. Beads were harvested by pulse centrifugation at 1,000 rpm, washed two times with PBS containing $500 \mathrm{mM} \mathrm{NaCl}$ and transferred to a column. Beads were washed with PBS containing $500 \mathrm{mM} \mathrm{NaCl}$ until no more proteins came down in the flow-through. Antibodies were eluted with $0.2 \mathrm{M}$ acetic acid, pH 2.7 containing $500 \mathrm{mM} \mathrm{NaCl}$ and $0.5 \mathrm{~mL}$ fractions were collected. Each fraction was immediately neutralized with $100 \mu \mathrm{L} 1 \mathrm{M}$ Tris/Base. To check for the presence of antibodies, $1 \mu \mathrm{L}$ of each fraction was spotted onto 
a nitrocellulose membrane, followed by staining with PonceauS. Protein containing fractions were combined and concentrated using a Spin-X UF concentrator (MWCO=30 kDa) to $\sim 0.5 \mathrm{~mL}$. The buffer was changed to PBS by repeated dilution with PBS and concentrating with the Spin-X UF concentrator. The concentrated antibody was mixed with one volume of $87 \%$ glycerol and stored at $-20^{\circ} \mathrm{C}$. The purified antibody was tested by Western blot (2.3.4) and indirect immunofluorescence (2.4.9).

\subsection{Cell Biology Techniques}

\subsubsection{Requirements for Sterile Working}

To prevent fungal or bacterial contaminations all cell culture work was performed under a laminar flow cabinet. Necessary tools and materials were either autoclaved and/or sterilized with $70 \%$ ethanol.

\subsubsection{Maintaining Cell Culture}

All cell lines were cultivated in Dulbecco's Modified Eagle Medium (DMEM) supplemented with 10\% fetal bovine serum (FCS), $2 \mathrm{mM} \mathrm{L-glutamine,} 100 \mathrm{U} / \mathrm{mL}$ penicillin and $100 \mu \mathrm{g} / \mathrm{mL}$ streptomycin at $37^{\circ} \mathrm{C}$ and $5 \% \mathrm{CO}_{2}$ in a humidified cell incubator. To guarantee optimal cell growth the medium was changed twice a week, often accompanied by cell sub-culturing.

\subsubsection{Sub-culturing of Adherent Cells}

To maintain cell proliferation, cells were sub-cultured upon reaching a confluency of $\sim 80 \%$. The overlying medium was aspirated, cells were washed once with 1x PBS and incubated with $1 \mathrm{~mL}$ trypsin at $37^{\circ} \mathrm{C}$ for optimal trypsin activity. Trypsin was inactivated by the addition of $9 \mathrm{~mL}$ of serum containing medium (Dulbecco's Modified Eagle Medium (DMEM) containing 10\% fetal bovine serum (FBS), $2 \mathrm{mM}$ L-glutamine and optionally $100 \mathrm{U} / \mathrm{mL}$ penicillin and $100 \mu \mathrm{g} / \mathrm{mL}$ streptomycin - in the following sections referred to as DMEM-10). For cell resuspension and dispersion of cell clumps, the cell suspension was rinsed several times over the bottom of the culture dish. Depending on the passage number of the cells and desired splitting ratio, a defined volume of the obtained cell suspension was transferred to a new culture dish and supplemented with DMEM-10 to a total volume of $10 \mathrm{~mL}$. The culture dish was pivoted gently to obtain an even distribution of cells and placed in a humidified cell incubator at $37^{\circ} \mathrm{C}$ and $5 \% \mathrm{CO}_{2}$. HeLa P4 cells were typically sub-cultivated two times per week and diluted in a ratio of 1:10 - 1:20. 


\subsubsection{Determination of Cell Concentration}

Cell numbers were determined by diluting $100 \mu \mathrm{L}$ of cell suspension in $10 \mathrm{~mL}$ of Coulter ISOTON II Diluent and counting with the CASY 1 system (Schärfe System).

\subsubsection{Coating of Cover Clips with Poly-L-Lysine}

Cover slips were treated with Poly-L-lysine to enhance the attachment of cells, due to the electrostatic interaction between negatively charged ions of the cell membrane and the positively charged Poly-L-Lysine. Cover slips were washed with 2-propanol, dried and coated with $0.01 \%(\mathrm{v} / \mathrm{v})$ Poly-L-Lysine followed by incubation for 30 minutes at room temperature. Unbound Poly-L-lysine was removed by washing two times with sterile water and dried cover slips were sterilized with UV light for 3 minutes at $0.12 \mathrm{~J} / \mathrm{cm}^{2}$.

\subsubsection{Calcium Phosphate Transfection of Mammalian Cells}

For transfection of mammalian cells with plasmid DNA using the calcium phosphate method, cells were seeded at 50,000 cells per well on cover slips in $500 \mu \mathrm{L}$ DMEM-10 into a 24-well plate format so that the cells had a confluence of $\sim 50 \%$ on the day of transfection. The next day $20 \mu \mathrm{L} \mathrm{CaCl}_{2}(250 \mathrm{mM})$ was added to $0.8 \mu \mathrm{g}$ of total plasmid DNA, mixed by vortexing at medium speed for 10 seconds, followed by a dropwise addition of $20 \mu \mathrm{L} 2 x$ HBS buffer (50 mM HEPES, $250 \mathrm{mM} \mathrm{NaCl}, 1.5 \mathrm{mM} \mathrm{Na} \mathrm{HPO}_{4}$, $\mathrm{pH}$ 6.98) and vortexing at maximum speed for 10 seconds. The mix was incubated for 20 minutes at room temperature to allow for the formation of calcium phosphate-DNA complexes prior addition to the cells. The cells were grown overnight in a humidified incubator at $37^{\circ} \mathrm{C}$ and $5 \% \mathrm{CO}_{2}$.

\subsubsection{Lipofectamine Transfection}

For transfection of mammalian cells with plasmid DNA using the Lipofectamine method, cells were seeded at 100,000 cells per well on a CS in $500 \mu \mathrm{L}$ DMEM-10 medium into a 24-well plate formats so that the cells had a confluence of $-70-90 \%$ on the day of transfection. Prior to transfection, the medium was changed to DMEM-10 medium without antibiotics. For transfection, $0.8 \mu \mathrm{g}$ of total plasmid DNA as well as $1 \mu \mathrm{L}$ of Lipofectamine ${ }^{\circledR}$ 2000 were diluted into $25 \mu \mathrm{L}$ Opti-MEM medium each. Both mixes were gently mixed and incubated for 5 minutes at room temperature. The diluted DNA was combined with the diluted Lipofectamine ${ }^{\circledR} 2000$, gently mixed and incubated for 20 minutes at room temperature to allow for the formation of nucleic acid-Lipofectamine complexes. The transfection mix was added dropwise to the cells and incubated for 4-5 hours in a 
Materials and Methods

humidified incubator at $37^{\circ} \mathrm{C}$ and $5 \% \mathrm{CO}_{2}$. The transfection medium was replaced with DMEM-10 containing antibiotics and incubated overnight in a humidified incubator at $37^{\circ} \mathrm{C}$ and $5 \% \mathrm{CO}_{2}$. For longer transfection times, the cells were sub-cultivated after 24 hours.

\subsubsection{RNA Interference (RNAi)}

For Lipofectamine transfection of siRNA, HeLa P4 cells were seeded at a density of 100,000 cells per 24 -well so that the cells had a density of $70-90 \%$ on the day of transfection. Prior to transfection, the medium was changed to $250 \mu \mathrm{L}$ DMEM-10 medium without antibiotics. For transfection, $2 \mu \mathrm{L}$ of Lipofectamine ${ }^{\circledR} 2000$ was added to $48 \mu \mathrm{L}$ of Opti-MEM and $1.75 \mu \mathrm{L}$ of siRNA $(20 \mu \mathrm{M})$ was added to $48.25 \mu \mathrm{L}$ of Opti-MEM medium and gently mixed. The Lipofectamine mix was incubated for 5 minutes at room temperature prior to addition to the siRNA mix. The transfection mix was gently mixed, incubated for 20 minutes at room temperature and added dropwise to the cells to a final siRNA concentration of $100 \mathrm{nM}$. The cells were incubated for 4 hours in a humidified incubator at $37^{\circ} \mathrm{C}$ and $5 \% \mathrm{CO}_{2}$ before the medium was changed to $500 \mu \mathrm{L}$ DMEM-10 containing antibiotics. After 24 hours cells were sub-cultivated on Poly-L-lysine coated cover slips and assayed for gene knock-down after 48-72 hours.

\subsubsection{Indirect Immunofluorescence}

For the detection of endogenous or tagged proteins transfected into mammalian cells, proteins were labeled by indirect immunofluorescence. Cells grown on cover slips were washed with PBS and fixed for 10 minutes with 3.7\% formaldehyde. For optional Hoechst staining of nuclei, the Hoechst dye $(10 \mathrm{mg} / \mathrm{mL})$ was diluted $1: 10,000$ into the fixation solution. The fixation solution was removed by washing two times with PBS, followed by permeabilization with $0.5 \%$ Triton $X-100$ on ice for 5 minutes. To reduce unspecific antibody staining, cells were blocked with $1 \%$ BSA, $10 \%$ FCS or $0.2 \%$ fish gelatin for 10 minutes. For the detection of the target protein, cover slips were transferred to a dark humidity chamber and incubated with the primary antibody (2.1.11) diluted in the respective blocking reagent for 1 hour, followed by three washes with PBS for 5-10 minutes each and addition of the secondary antibody (2.1.11) diluted in the blocking reagent for 1 hour. Unbound antibodies were washed away by three washes with PBS for 5-10 minutes each, followed by a final quick wash with water. Cover slips were dried and mounted with Dako Fluorescent Mounting Medium or with Mowiol mounting medium containing $1 \mu \mathrm{g} / \mathrm{mL}$ DAPI for staining of the cell nuclei. 


\subsubsection{Proximity Ligation Assays (PLA)}

The Duolink ${ }^{\circledR}$ in situ Proximity Ligation Assay kit was used to detect proteins in close proximity in single cells with a maximum theoretical distance of $40 \mathrm{~nm}$. HeLa P4 cells were seeded on poly-L-lysine coated cover slips (2.4.5) in a 24-well plate at a concentration of 45,000 cells/well. Cells were allowed to attach to the cover slips for a minimum of 48 hours prior to fixation. Cells were washed three times with PBS, fixed with $3.7 \%$ formalin for 30 minutes, washed three times with PBS and were permeabilized with 0.5\% Triton X-100 for 5 minutes at room temperature. After washing three more times with PBS, the cells were blocked with Duolink ${ }^{\circledR}$ blocking solution for 30 minutes in a humidity chamber preheated to $37^{\circ} \mathrm{C}$. Primary antibodies (2.1.11) of two different species that bind to the proteins to be detected were diluted in $50 \mu \mathrm{L}$ Duolink ${ }^{\circledR}$ antibody diluent and were added to each sample for one hour at room temperature. The slides were washed two times with $120 \mu \mathrm{L}$ PLA Wash Buffer A for 5 minutes with gentle agitation and the secondary antibodies conjugated with oligonucleotides (PLA probe MINUS and PLA probe PLUS) were diluted 1:5 in Duolink ${ }^{\circledR}$ antibody diluent. Cells were incubation with the secondary antibodies in a pre-heated humidity chamber for 1 hour at $37^{\circ} \mathrm{C}$. Unbound secondary antibodies were removed by washing two times with $120 \mu \mathrm{L}$ PLA Wash Buffer A for 5 minutes with gentle agitation. The PLA probe oligonucleotides were linked in a ligation reaction by addition of a ligation solution consisting of ligase and two oligonucleotides that can hybridize to the two PLA probes and join them to form a closed circle if they are in close proximity. Ligation was done in a humidity chamber for 30 minutes at $37^{\circ} \mathrm{C}$, followed by two wash steps with $120 \mu \mathrm{L}$ PLA Wash Buffer A for 2 minutes with gentle agitation and incubation with the amplification solution for 100 minutes at $37^{\circ} \mathrm{C}$. The amplification solution consists of a polymerase, nucleotides and fluorescently labeled oligonucleotides and allows for rolling circle amplification of the ligated circle template, generating a concatemeric product. By hybridization of fluorescently labeled oligonucleotides to the concatemer, proteins in close proximity are visualized as distinct fluorescent spots. Slides were washed two times with $120 \mu \mathrm{L}$ PLA Wash Buffer B for 10 minutes to remove the amplification solution. If required, a counterstaining step (see below) was included after this step to detect the proteins of interest or an indirect immunofluorescence (2.4.9) was performed starting from the blocking step. If no counterstaining or indirect immunofluorescence staining was done, the slides were washed with $120 \mu \mathrm{L} 0.01 \mathrm{x}$ PLA Wash Buffer B for 1 minute. Coverslips were mounted with Duolink ${ }^{\circledR}$ in situ mounting medium (contains DAPI), sealed with nail polish and stored at $-20^{\circ} \mathrm{C}$. Cover slips were 
imaged with the Confocal microscope LSM 510 META and PLA dots were quantified with CellProfiler (2.4.13).

\section{Counterstaining Method}

After removal of the amplification-polymerase solution, the cover slips were washed two times with Wash Buffer B for 10 minutes, followed by one wash step with Wash Buffer A for 1 minute. The samples were incubated with the primary antibody (50 $\mu \mathrm{L} /$ well) diluted in Duolink $^{\circledR}$ antibody diluent for 40 minutes. Optionally, a FITC-Phalloidin antibody $(0.2 \mathrm{mg} / \mathrm{mL})$ was added at a 1:600 dilution to delineate the entire cell by staining for filamentous actin. After washing for 1 minute with Wash Buffer $A$, the cells were incubated with the secondary antibody diluted in Duolink ${ }^{\circledR}$ antibody diluent for 30 minutes. The cover slips were washed two times with Wash Buffer A for 2 minutes and once with 0.01x Wash Buffer B for 1 minute prior to mounting as described above. All steps were performed at room temperature.

\subsubsection{Transport Assay}

The transport assay is based on a method developed by Adam et al., 1990 (65). HeLa P4 cells were grown on Poly-L-lysine coated cover slips to $60-80 \%$ confluence. Cells were washed twice with ice-cold transport buffer (20 mM HEPES, $110 \mathrm{mM}$ KOAc, $2 \mathrm{mM}$ $\mathrm{Mg}(\mathrm{OAc})_{2}, 1 \mathrm{mM}$ EGTA, pH 7.3, $2 \mathrm{mM}$ DTT, $1 \mu \mathrm{g} / \mathrm{mL}$ of each AP and LP) and permeabilized with $0.007 \%$ digitonin on ice for 3-5 minutes. At low concentrations, digitonin selectively permeabilizes the cholesterol-rich plasma membrane and leaves other membranes lower in cholesterol such as the nuclear envelope intact $(179,180)$. Permeabilization efficiency was checked by Trypan blue staining. Cytosolic proteins were washed out by washing three times for 3 minutes with TPB and the permeabilized cells were incubated with $20 \mu \mathrm{L}$ import reaction for 30 minutes at $30^{\circ} \mathrm{C}$ in a humidity chamber or at $4^{\circ} \mathrm{C}$ on ice. The import mix consisted of $500 \mathrm{nM}$ import substrate, $500 \mathrm{nM}$ import receptor, $4 \mu \mathrm{M}$ Ran, $0.5 \mu \mathrm{L}$ ATP regenerating system (1 mM ATP, $5 \mathrm{mM}$ creatine phosphate, $20 \mathrm{U} / \mathrm{mL}$ creatine phosphokinase), $2 \mathrm{mg} / \mathrm{mL}$ BSA in transport buffer and optionally $8 \mu \mathrm{L}$ cytosol $(9 \mathrm{mg} / \mathrm{mL})$. The import reaction was stopped by washing three times for 3 minutes with TPB and fixation with 3.7\% formaldehyde in PBS for 10 minutes. Proteins were detected by indirect immunofluorescence (2.4.9). 
Materials and Methods

\subsubsection{Confocal Microscopy}

Fluorescent samples were analyzed with an Axiovert $200 \mathrm{M}$ inverted microscope with a 63x Plan-Neofluar 1.3 NA water-corrected objective using the water-based Immersol W 2010 immersion oil and the X-cite 120 mercury lamp as a light source. Confocal images were acquired with the Zeiss LSM 510 META confocal imaging system. The confocal imaging system is equipped with five different laser lines that allow for excitation of various fluorophores. The Diode laser $405 \mathrm{~nm}$ was used for excitation of Hoechst 33258 or DAPI, the tunable Argon 458/477/488/514 nm laser for GFP, CFP and the AlexaFluor488 dye, the HeNe $543 \mathrm{~nm}$ laser for YFP, the HeNe $594 \mathrm{~nm}$ laser for mCherry and AlexaFluor594 dye and the HeNe 633 nm laser for AlexaFluor633 or AlexaFluor647 dye. To reduce crosstalk between different channels, the Multi Track image acquisition mode was used in the LSM 5 software. Laser transmission was adjusted depending on the fluorescence signal intensity and to minimize bleaching effects. Typically the pinhole was set to a diameter of 1 airy unit, but if required, was changed to 0.8-2.0 airy units to increase signal intensity or to have the same optical slice thickness for co-localization studies. Image intensity and background level gains were controlled with the Detector Gain and the Amplifier Offset, respectively, and adjusted with the help of the range indicator function in the palette tool. To increase the signal to noise ratio, four images were averaged at a data depth of 8 bit and a scan speed of 1.28-3.2 $\mu$ s/pixel was used. Acquired images were edited with the Fiji software. If necessary, the brightness and contrast were linearly enhanced for printing.

\subsubsection{CellProfiler}

The CellProfiler software (version 2.1.1) was used for the quantification of in situ proximity ligation assay (PLA) interactions. A pipeline was generated to count the number of PLA interactions in the whole cell, the cytoplasm and the nucleus. Cell nuclei were identified with the "IdentifyPrimaryObject" module using DAPI images. The typical diameter of the objects was set to 80-150 pixels and two-class Otsu adaptive thresholding was applied. Clumped objects were distinguished based on intensity values and the smoothing filter for declumping and the minimum allowed distance between local maxima was set to be calculated automatically. The cellular area was demarcated with the "IdentifySecondaryObjects" module using the Distance - N method by expanding the identified nuclear area by 80 pixels. Using the "IdentifyTertiaryObjects" the nuclear area was then subtracted from the cell area to obtain the cytoplasm. Cells touching the border of an image were excluded from further analysis. PLA fluorescent dots were identified with the "IdentifyPrimaryObjects" module using PLA images. The typical diameter of the PLA 
objects was set to 2-10 pixels for the PLA objects and two-class Otsu adaptive thresholding was used with a minimum threshold of 0.15 . Clumped objects were separated as described for the identification of cell nuclei. PLA dots in the nucleus and the cytoplasm were distinguished with the help of the "Masklmage" module. With the "RelateObjects" module the identified PLA objects in the cell, the nucleus and the cytoplasm were related back to the previously identified cell, nuclear and cytoplasmic area, respectively. Data was exported with the "ExportToSpreadsheet" module and visualized with the GraphPad Prism software (version 5.01).

\subsection{SILAC and Mass Spectrometry}

\subsubsection{Dialyzed FCS}

FCS was dialyzed against PBS using a dialysis membrane (Spectra/Por ${ }^{\circledR}$ Dialysis Membrane) with a molecular weight cut-off of $8 \mathrm{kDa}$. PBS was changed three times after 1 hour, overnight and again after 1 hour incubation at $4^{\circ} \mathrm{C}$ under constant stirring. The dialyzed FCS was sterile aliquoted and frozen at $-20^{\circ} \mathrm{C}$.

\subsubsection{Metabolic Labeling of HeLa P4 Cells}

For SILAC experiments, HeLa P4 cells were grown in DMEM, high glucose, no glutamine, no lysine, no arginine supplemented with 10\% dialyzed FCS, 6 mM L-glutamine, $100 \mathrm{U} / \mathrm{mL}$ penicillin, $100 \mu \mathrm{g} / \mathrm{mL}$ streptomycin and labeled or unlabeled L-lysine and L-arginine at final concentrations of $73 \mathrm{mg} / \mathrm{L}$ and $42 \mathrm{mg} / \mathrm{L}$, respectively. For light SILAC media, DMEM, high glucose, no glutamine, no lysine, no arginine was mixed in a 1:1 ratio with DMEM, high glucose, which contains light L-lysine (Lys0) and L-arginine (Arg0) at final concentrations of $146 \mathrm{mg} / \mathrm{L}$ and $84 \mathrm{mg} / \mathrm{L}$, respectively. The medium SILAC media was supplemented with 4,4,5,5-D4-L-Lysine $2 \mathrm{HCl}$ (Lys4) and ${ }^{13} \mathrm{C}_{6}$-L-arginine $\mathrm{HCl}$ (Arg6) and the heavy SILAC media with ${ }^{13} \mathrm{C}_{6}{ }^{15} \mathrm{~N}_{2}$-L-lysine $\mathrm{HCl}$ (Lys8) and ${ }^{13} \mathrm{C}_{6}{ }^{15} \mathrm{~N}_{4}$-L-arginine $\mathrm{HCl}$ (Arg10). To achieve labeling efficiency of more than 95\% cells were allowed to undergo at least five doublings. Typically, cells were subcultivated every 2-3 days with the appropriate SILAC media for a total of five passages at $37^{\circ} \mathrm{C}$ and $5 \% \mathrm{CO}_{2}$ in a humidified cell incubator prior to harvesting. 


\subsubsection{Binding Assay with Labeled HeLa P4 Cells}

\section{Preparation of Phenyl-Sepharose}

Phenyl-Sepharose (80 $\mu \mathrm{L} /$ reaction) was washed once each with deionized water and transport buffer (20 mM HEPES, $110 \mathrm{mM}$ KOAc, 2 mM Mg(OAc) $)_{2}, 1$ mM EGTA, pH 7.3, $2 \mathrm{mM}$ DTT, $1 \mu \mathrm{g} / \mathrm{mL}$ of each AP and LP) containing $10 \mathrm{mg} / \mathrm{mL}$ BSA. After blocking in the same buffer with rotation for 30 minutes at $4^{\circ} \mathrm{C}$, the beads were washed once with transport buffer containing $10 \mathrm{mg} / \mathrm{mL}$ BSA and used for pre-clearing of cell lysates.

\section{Preparation of Affinity Matrix}

IgG-Sepharose (20 $\mu \mathrm{L} /$ reaction) was washed once each with deionized water and transport buffer (20 mM HEPES, $110 \mathrm{mM} \mathrm{KOAc,} 2$ mM Na${ }_{2} \mathrm{CO}_{3}, 1 \mathrm{mM}$ EGTA, pH 7.3, $2 \mathrm{mM}$ DTT, $1 \mu \mathrm{g} / \mathrm{mL}$ of each AP and LP) containing $10 \mathrm{mg} / \mathrm{mL}$ BSA, followed by blocking in the same buffer with rotation for 30 minutes at $4^{\circ} \mathrm{C}$. The beads were washed once in the same buffer and $0.5 \mathrm{nmol}$ Hzz-importin 13 was immobilized to $20 \mu \mathrm{L}$ blocked IgG-Sepharose rotating for one hour at $4^{\circ} \mathrm{C}$ in a total volume of $500 \mu \mathrm{L}$ in transport buffer containing $10 \mathrm{mg} / \mathrm{mL}$ BSA. The beads were pelleted by centrifugation at $300 \times \mathrm{g}$ for 2 minutes and washed three times with $1 \mathrm{~mL}$ transport buffer. The Hzz-importin 13 affinity was pre-incubated with either $10 \mu \mathrm{M}$ RanGTP Q69L $5 \mu \mathrm{M}$ Ubc9 or transport buffer alone in a total volume of $500 \mu \mathrm{L}$ rotating for one hour at $4^{\circ} \mathrm{C}$. The beads were washed three times with $1 \mathrm{~mL}$ transport buffer prior to assembly of the binding reactions.

\section{Preparation of Cell Lysate}

For SILAC binding experiments, two 15-cm HeLa P4 cell plates per labeling condition were washed two times with PBS and coated with $2 \mathrm{~mL}$ trypsin. The trypsin was aspirated and the plates were incubated at $37^{\circ} \mathrm{C}$ until the cells started to detach. The labeled cells were collected separately in PBS containing 10\% dialyzed FCS to neutralize the trypsin and washed three times with transport buffer to remove any residual FCS and trypsin. The cells were counted with the CASY 1 system (Schärfe System), adjusted to $1 \times 10^{8}$ cells $/ \mathrm{mL}$ in transport buffer (20 mM HEPES, 110 mM KOAc, 2 mM Mg(OAc), 1 mM EGTA, pH 7.3, $2 \mathrm{mM}$ DTT, $1 \mu \mathrm{g} / \mathrm{mL}$ of each AP and LP and $1 \mathrm{x}$ Roche complete protease inhibitor cocktail) and transferred to a $1.5 \mathrm{~mL}$ Protein LoBind Tube. The cells were permeabilized with $0.07 \%$ digitonin on ice for $3-5$ minutes by adding $7 \mu \mathrm{L}$ of $10 \%$ digitonin per $1 \times 10^{8}$ cells. Permeabilization efficiency was checked under the microscope by mixing $1 \mu \mathrm{L}$ of lysate with $10 \mu \mathrm{L}$ Trypan blue. If necessary, the digitonin concentration was slightly increased 
until more than $95 \%$ of the cells were permeabilized. Cell lysates were cleared by centrifugation at $1,500 \times \mathrm{g}$ for 15 minutes to remove nuclei and cell debris, followed by ultracentrifugation in the Centrifuge Optima MAX-XP with the rotor TLA100.3 at $100,000 \times \mathrm{g}, 4^{\circ} \mathrm{C}$ for 30 minutes. Two reduce unspecific binding to the affinity matrix, each cell lysate from 2x 15-cm plates was pre-incubated with $60 \mu \mathrm{g} \mathrm{Hzz}$ immobilized to $20 \mu \mathrm{L}$ blocked IgG-Sepharose, as well as, $80 \mu \mathrm{L}$ blocked Phenyl-Sepharose to deplete the cell extract of nuclear transport receptors, with rotation for two hours at $4^{\circ} \mathrm{C}$. The beads were pelleted by centrifugation for 2 minutes at $300 \times \mathrm{g}$ and the supernatant was collected for the binding reaction. A sample of both the uncleared and cleared lysate was taken for later analysis.

\section{Binding Reaction}

For binding reactions, $20 \mu \mathrm{L}$ of Hzz-importin 13 affinity matrix was incubated with $350 \mu \mathrm{L}$ HeLa P4 lysate from two 15-cm plates (precleared with IgG-Hzz and Phenyl-Sepharose) and either $10 \mu \mathrm{M}$ RanGTP ${ }_{\mathrm{Q} 69 \mathrm{~L}}, 5 \mu \mathrm{M}$ Ubc9 or transport buffer alone in a final volume of $500 \mu \mathrm{L}$ rotating for two hours at $4^{\circ} \mathrm{C}$. Beads were pelleted at $800 \times \mathrm{g}$ for 2 minutes, an unbound sample was collected for later analysis and the beads were washed with one quick wash and one 5 minute wash with transport buffer. After this step, all beads were combined for the last washing step and bound proteins were eluted together with $150 \mu \mathrm{L}$

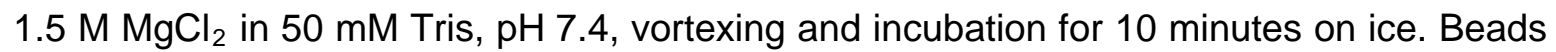
were pelleted by centrifugation and the supernatant was collected and transferred to a new tube. Proteins were precipitated by addition of $1350 \mu \mathrm{L} 100 \%$ isopropanol, vortexing and incubation for 10 minutes on ice, followed by centrifugation for 20 minutes at 14,000 x $\mathrm{g}$. The supernatant was removed and the protein pellet was dried prior to reconstitution in 60 $\mu \mathrm{L} 4 \mathrm{x}$ SDS-sample buffer. The magnesium chloride eluted beads were washed two times with transport buffer and a second elution was done with $60 \mu \mathrm{L} 4 \mathrm{x}$ SDS-sample buffer to identify remaining proteins bound to the affinity matrix. All eluates were boiled at $95^{\circ} \mathrm{C}$ for 10 minutes and analyzed by SDS-PAGE, followed by Coomassie staining (2.3.2), silver staining (2.3.3) or Western blotting (2.3.4).

\subsubsection{In-Gel Tryptic Digestion of Proteins}

One-third of the magnesium chloride eluate from the importin 13 binding reaction was loaded onto a 12\% SDS gel. The SDS-PAGE (2.3.1) was stopped after the samples had entered one-quarter of the stacking gel. The gel was washed with deionized water, fixed for one hour with fixation solution (40\% ethanol, $10 \%$ acetic acid) and washed two times 
for 10 minutes with deionized water. Each sample lane was split into 6 parts, cut into $2 \mathrm{~mm}$ sized pieces and transferred to low-bind Eppendorf cups. In-gel digestion was performed based on a protocol provided by Dr. Oliver Valerius (Universität Göttingen), which in turn was adapted from Shevchenko et al., 1996 (181). All solutions were prepared with LC/MS Grade Optima ${ }^{\text {TM }}$ Water. In brief, gel pieces were dehydrated with $50 \mu \mathrm{L}$ acetonitrile and dried in a SpeedVac Concentrator (Vacuum Christ Alpha 1-4, W. Krannich) for 10 minutes after removal of the acetonitrile. Gel pieces were rehydrated in $150 \mu \mathrm{L} 10 \mathrm{mM}$ DTT, $100 \mathrm{mM} \mathrm{NH}_{4} \mathrm{CO}_{3}$ to reduce disulfide bonds for 1 hour at $56^{\circ} \mathrm{C}$. The DTT solution was removed and resulting free cysteine thiol groups were alkylated with $150 \mu \mathrm{L} 55 \mathrm{mM}$ iodoacetamide, $100 \mathrm{mM} \mathrm{NH}_{4} \mathrm{CO}_{3}$ for 45 minutes at room temperature in the dark. Gel pieces were washed with $150 \mu \mathrm{L} 100 \mathrm{mM} \mathrm{NH}_{4} \mathrm{CO}_{3}$ and then dehydrated with $150 \mu \mathrm{L}$ acetonitrile both times shaking at $1,000 \mathrm{rpm}$ for 10 minutes at room temperature. The washing (rehydration of gel) and dehydration steps were repeated once and then the gel pieces were dried for 10 minutes in a SpeedVac after removal of the acetonitrile. Gel pieces were rehydrated in $50 \mu \mathrm{L}$ trypsin digestion buffer $(20 \mu \mathrm{g} / \mathrm{mL}$ trypsin (Serva), prepared according to the manufacturer's instructions) for 45 minutes on ice. The digestion buffer was discarded and gel pieces were completely covered with $50 \mu \mathrm{L} 25 \mathrm{mM} \mathrm{NH}_{4} \mathrm{CO}_{3}$. Tryptic peptide digestion was allowed to occur overnight at $37^{\circ} \mathrm{C}$.

\subsubsection{Extraction of Peptides}

Following trypsin digestion, supernatants were collected by centrifugation for 1 minute at 13,000 rpm (Centrifuge 5415R, Eppendorf) and transferred to new tubes. Residual acidic peptides were extracted from the gel pieces by incubation with $50 \mu \mathrm{L} 20 \mathrm{mM} \mathrm{NH}_{4} \mathrm{CO}_{3}$ shaking at 1,000 rpm for 10 minutes at room temperature. Residual basic peptides were extracted three times by incubation with $50 \mu \mathrm{L} 50 \%$ acetonitrile, 5\% formic acid shaking at $1,000 \mathrm{rpm}$ for 20 minutes at room temperature. All supernatants were collected by centrifugation for 1 minute at $13,000 \mathrm{rpm}$, pooled and completely dried by vacuum evaporation in a SpeedVac. Samples were either stored at $-20^{\circ} \mathrm{C}$ or directly desalted over C18 stage tips (2.5.6). Peptide extraction was performed based on a protocol provided by Dr. Oliver Valerius (Universität Göttingen), which in turn was adapted from Shevchenko et al., 1996 (181).

\subsubsection{Desalting of Peptides}

Peptides were desalted by purification over C18 stage tips following a protocol provided by Dr. Oliver Valerius (Universität Göttingen), which in turn is based on a tutorial provided by 
the group of Prof. Dr. Matthias Mann (http://www.biochem.mpg.de/226863/Tutorials). In brief, C18 stage tips were assembled by cutting out small circles of C18 material (Empore $^{\mathrm{TM}} \mathrm{C} 1847 \mathrm{~mm}$ Extraction Disc, 3M Company) and placing two on top of each other into the tip of a $200 \mu \mathrm{L}$ pipette tip. C18 stage tips were activated with $100 \mu \mathrm{L} 100 \%$ methanol, $0.1 \%$ formic acid and washed with $100 \mu \mathrm{L} \mathrm{70 \%} \mathrm{acetonitrile,} 0.1 \%$ formic acid, followed by two wash steps with $100 \mu \mathrm{L} \mathrm{H}_{2} \mathrm{O}, 0.1 \%$ formic acid by centrifugation for 2 minutes at maximum speed. Simultaneously, dried peptide pellets were resuspended in $20 \mu \mathrm{L}$ fresh sample buffer $\left(98 \% \mathrm{H}_{2} \mathrm{O}, 2 \%\right.$ acetonitrile, $0.1 \%$ formic acid) by shaking for 10 minutes at 1,000 rpm and incubation in a Decon FS-100 ultrasonic bath at maximum power for 3 minutes. Samples were loaded onto the C18 stage tips and brought in contact with the C18 material by brief centrifugation for 5 seconds at 1,000 rpm (Centrifuge 5415R, Eppendorf). After incubation for 5 minutes, samples were centrifuged for 5 minutes at 4,000 rpm. To increase the peptide yield samples were reloaded and centrifuged again for 5 minutes at 4,000 rpm. At this step, all peptides should be bound to the C18 material, therefore the flow-through was discarded. The C18 column was washed twice with $100 \mu \mathrm{L}$ $\mathrm{H}_{2} \mathrm{O}, 0.1 \%$ formic acid by centrifugation for 2 minutes at 10,000 rpm. For peptide elution, the C18 stage tip was transferred to a $1.5 \mathrm{~mL}$ low-bind Eppendorf cup and bound peptides were eluted with $60 \mu \mathrm{L} 70 \%$ acetonitrile, $0.1 \%$ formic acid by centrifugation for 5 minutes at 4,000 rpm. Peptide eluates were dried completely in the SpeedVac and peptide pellets were stored at $-20^{\circ} \mathrm{C}$ until liquid chromatography-coupled-mass spectrometry (LC-MS) analysis of peptides.

\subsubsection{Liquid Chromatography-Coupled-Mass Spectrometry (LC-MS) Analysis of Peptides}

Prior to the analysis of peptides with nanoflow liquid chromatography (UltiMate ${ }^{\mathrm{TM}} 3000$ RSLCnano System, Thermo Scientific) coupled to nano electrospray ionization (nESI) mass spectrometry, dried peptide pellets were solved in $20 \mu \mathrm{L}$ sample buffer $\left(98 \% \mathrm{H}_{2} \mathrm{O}\right.$, $2 \%$ acetonitrile, $0.1 \%$ formic acid) by shaking for 10 minutes at 1,000 rpm. Samples were incubated for 3 minutes at maximum power in an ultrasonic bath and transferred to LC-MS vials (Grace). Sample solutions of 1 to $2 \mu \mathrm{L}$ were loaded and washed on an Acclaim ${ }^{\mathrm{TM}}$ PepMap $^{\text {тм }} 100$ pre-column $(100 \mu \mathrm{m} \times 2$ cm, C18, $5 \mu \mathrm{m}, 100 \AA$, Thermo Scientific) in 100\% solvent A (98\% water, $2 \%$ acetonitrile, $0.07 \%$ trifluoroacetic acid) at a flow rate of $25 \mu \mathrm{L} / \mathrm{min}$ for 6 minutes. Peptides were separated by reverse phase chromatography, on an Acclaim ${ }^{\mathrm{TM}}$ PepMap $^{\mathrm{TM}}$ RSLC column $(75 \mu \mathrm{m} \times 50 \mathrm{~cm}, \mathrm{C} 18,2 \mu \mathrm{m}, 100 \AA$, Thermo Scientific). A first gradient was run from $98 \%$ solvent $A$ (water, $0.1 \%$ formic acid) and $2 \%$ 
solvent B (80\% acetonitrile, $20 \%$ water, $0.1 \%$ formic acid) to $32 \%$ solvent B within 110 minutes, followed by a second gradient from 32\% to $65 \%$ solvent B within 16 minutes at a flow rate of $300 \mathrm{~nL} / \mathrm{min}$ (solvents and acids from Fisher Chemicals). Peptides eluting from the chromatography were on-line ionized by nESI using the Nanospray Flex Ion Source (Thermo Scientific) at $2.4 \mathrm{kV}$ and continuously transferred into the Orbitrap Velos Pro $^{\mathrm{TM}}$ Nano ESI Mass Spectrometer, (Thermo Scientific). Full scans were acquired with the Orbitrap-FT analyzer in the mass range of $300-1,850 \mathrm{~m} / \mathrm{z}$ and with a resolution of 60,000 . The LTQ Velos Pro linear ion trap (CID) was used for parallel data-dependent top 10 fragmentation. Programming of LC-MS methods and data acquisition was done with the XCalibur 2.2 software (Thermo Scientific). All LC-MS analyses of peptides were performed by the Service Unit LCMS Protein Analytics (Dr. Oliver Valerius and Dr. Kerstin Schmitt), Georg-August-Universität Göttingen.

\subsubsection{Analysis of Mass Spectrometry Data}

Raw mass spectrometry files were analyzed with the MaxQuant 1.5.1.0 software in the case of quantitative SILAC data or the Proteome Discoverer Software version 1.4 in the case of all other mass spectrometry analyses. In the case of the Proteome Discoverer, mass spectrometry data was searched against the NCBI-derived 'homo sapiens' protein database including gb, ref, dbj, emb, and pdb entries (status 09/14/2016, 124176 sequences). Search parameters were set as follows: trypsin digestion mode with the maximum of two missed cleavage sites, oxidation of methionine and $\mathrm{N}$-terminal protein acetylation were set as variable modifications and carbamidomethylation of cysteine as fixed modification. The mass tolerance for the peptide precursors was $10 \mathrm{ppm}$ and $0.6 \mathrm{Da}$ for fragment ions. False discovery rates were calculated with Proteome Discoverer, using the reverse-decoy mode, and the filter for valid peptide sequence matches was set to high confidence (FDR $\leq 0.01$ ). The Proteome Discoverer search was performed by the Service Unit LCMS Protein Analytics (Dr. Oliver Valerius and Dr. Kerstin Schmitt), Georg-AugustUniversität Göttingen.

In the case of quantitative data analysis with the MaxQuant software, mass spectrometry data was analyzed against the human protein database derived from Uniprot (Proteome ID: UP000005640, 71,913 entries, download 2016). Search parameters were set as follows: trypsin/P digestion mode (tryptic specificity with no proline restriction) with maximum of two missed cleavages, oxidation of methionine and $\mathrm{N}$-terminal protein acetylation were set as variable modifications and carbamidomethylation of cysteine was set as a fixed modification. Arg6 and Lys4 were defined as medium peptide labels and 
Arg10 and Lys8 as heavy peptide labels. The mass tolerance for peptide precursors was $4.5 \mathrm{ppm}$ and for fragment ions 0.5 Da. Fourier transform-based mass spectrometer (FTMS) requantification and FTMS recalibration were enabled. Protein quantification was performed with a minimum ratio count of two and unique plus razor peptides were considered. False discovery rates were calculated by MaxQuant, using the revert-decoy mode and the filter for valid peptide sequence matches was set to 0.01. MaxQuant output data was further processed using Perseus software 1.5.0.15. Gene Ontology (GO) analysis was done using DAVID Bioinformatics Resources 6.8 (182, 183). Only GO terms for biological processes were considered (GOTERM_BP_ALL). 


\section{Results}

Until recently, only a limited number of importin 13 cargoes had been identified. To expand the range of known importin 13 import and specifically export cargoes, three different approaches were applied. These included an importin 13 overexpression screen using a library of nuclear proteins, an importin binding experiment followed by mass spectrometry and a SILAC based proteomics approach coupled to an importin 13 binding assay that allowed for the distinction of importin 13 import and export cargoes. Further, various cellular and biochemical assays were established with known importin 13 cargoes, to allow for analysis of newly identified importin 13 substrate candidates.

\subsection{Characterization of Importin 13 and Established Cargoes}

\subsubsection{Importin 13 Expression Levels Are Low in Different Human Cancer Cell Lines}

To define the endogenous importin 13 expression levels in different cancer cell lines and determine whether importin 13 may be a limiting factor in nucleocytoplasmic transport, the cellular concentration of importin 13 in three different human cancer cell lines was determined. For this, the cell lysates of a defined number of HeLa P4, U2OS and 293T HEK cells were loaded onto an SDS gel together with known amounts of purified His-importin 13 and analyzed by Western blot (Figure 4).

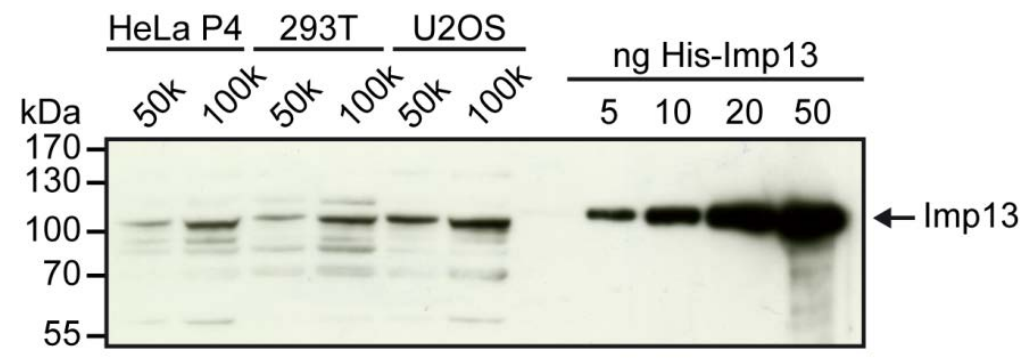

Figure 4: Endogenous importin 13 concentrations in cancer cell lines. Defined amounts of HeLa P4, 293T HEK and U2OS cells (50,000 and 100,000 cells) were lysed in SDS sample buffer and analyzed by SDS-PAGE together with 5, 10, 20 and $50 \mathrm{ng}$ of purified His-tagged importin 13, followed by immunoblotting with an anti-importin 13 antibody. Assuming a cell volume of $\sim 2,500 \mu \mathrm{m}^{3}$ an importin 13 concentration in the range of $110-310 \mathrm{nM}$ was determined for all three cancer cell lines. 
Based on the assumption that these cells have a volume of $\sim 2,500 \mu \mathrm{m}^{3}$ (184), a cellular importin 13 concentration of $\sim 110 \mathrm{nM}$ was determined for HeLa P4 cells, $\sim 120 \mathrm{nM}$ for 293T HEK cells and $\sim 310 \mathrm{nM}$ for U2OS cells. In comparison, other transport receptors have been estimated to have cellular concentrations between 1-4 $\mu \mathrm{M}(77,185)$. This shows that at least in the analyzed cell lines importin 13 is not an abundant protein and therefore could be rate limiting for transport.

\subsubsection{Importin 13 Is Rate Limiting in HeLa P4 Cells}

To test whether endogenous importin 13 is indeed rate limiting in HeLa P4 cells, the effect of importin 13 overexpression on the subcellular localization of the endogenous export cargo elF1A was analyzed. Cells transfected with plasmids coding for FLAG-importin 13 or mock transfected cells were immunostained for endogenous elF1A with an anti-elF1A antibody (Figure 5). In mock transfected cells, elF1A showed a predominantly nuclear localization and a weak cytoplasmic signal, while in cells overexpressing FLAG-importin 13 most of the nuclear signal was lost. Thus, importin 13 is rate limiting for elF1A export in HeLa P4 cells.

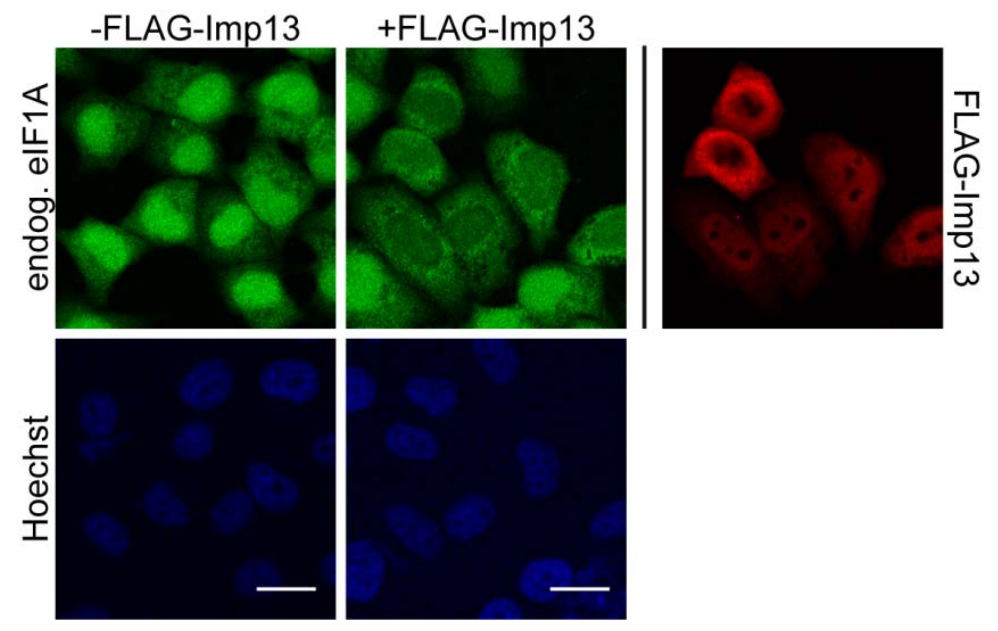

Figure 5: Importin 13 is rate limiting for export of endogenous elF1A. HeLa P4 cells were transiently transfected with plasmids coding for FLAG-importin 13 or mock transfected using the calcium phosphate method and immunostained with an anti-eIF1A antibody and anti-FLAG antibody. The scale bars correspond to $20 \mu \mathrm{m}$. 


\subsubsection{Importin 13 Affects the Subcellular Localization of elF1A and Ubc9}

Grünwald et al., 2013 (107) demonstrated that only cells expressing importin 13 wild type, but not the importin 13 mutant Glu436Arg/Asp481Arg, can export elF1A-GFP in vivo. To test whether these findings can be repeated and to establish elF1A as a positive control for importin 13 mediated nuclear export, importin 13 overexpression experiments were performed. In addition, the effect of importin 13 on the known importin 13 import cargo Ubc9 was analyzed, to establish Ubc9 as a positive control for importin 13 mediated nuclear import. In order to do this, HeLa P4 cells were co-transfected with plasmids coding for Ubc9 or elF1A and importin 13 or an empty control vector and analyzed for any changes in subcellular distribution (Figure 6). As previously reported (107), GFP-tagged elF1A was mainly localized in the nucleus and showed strong nucleoli enrichment but was strongly depleted from these sites upon importin 13 coexpression. In contrast, mutant importin 13-E436R/D481R did not affect the subcellular localization of elF1A-GFP.

Further, to determine whether the size of elF1A affects its subcellular localization, elF1A was fused to an N-terminal GFP-GST-tag. This fusion construct (72 kDa) should be well above the diffusion limit of the NPC and as a consequence GFP-GST-elF1A should only be able to enter the nucleus with the help of $\beta$-karyopherins, but not by diffusion. Indeed, GFP-GST-elF1A showed a cytoplasmic localization in the absence of importin 13, supporting the hypothesis that elF1A enters the nucleus by passive diffusion due to its small size (16.5 kDa) (135). However, it cannot be entirely excluded that nuclear import of GFP-GST-eIF1A is impeded due the fusion-tag. Note that the GFP-GST-tag was fused to the N-terminus, while the GFP-tag was fused to the C-terminus of elF1A. In summary, the above observations are in agreement with the previous reports $(107,135)$ that elF1A enters the nucleus by passive diffusion and is exported back out of the nucleus by importin 13, while the importin 13 mutant Glu436Arg/Asp481Arg is impaired in elF1A export.

To establish a positive control for importin 13 mediated nuclear import, the subcellular localization of Ubc9 fused to both a GFP-GST- and a HA-tag was analyzed. While HA-Ubc9 by itself already showed a nuclear localization and consequently was not affected by importin 13 coexpression, GFP-GST-Ubc9 was too large to efficiently enter the nucleus on its own and required coexpression of FLAG-importin 13 to localize to the nucleus. This suggests that HA-Ubc9 ( $19 \mathrm{kDa})$ as elF1A can enter the nucleus by passive diffusion due to its small size. Further, importin 13 has been shown to bind its cargoes Ubc9 and elF1A at non-overlapping binding sites (107) and indeed the importin 13 mutant impaired in elF1A export was still fully functional in mediating import of GFP-GST-Ubc9 (107). 

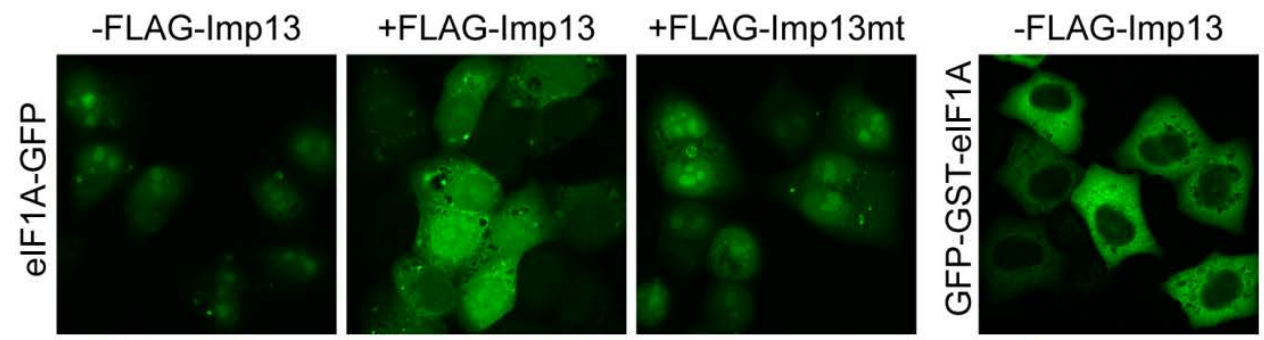

+FLAG-Imp13
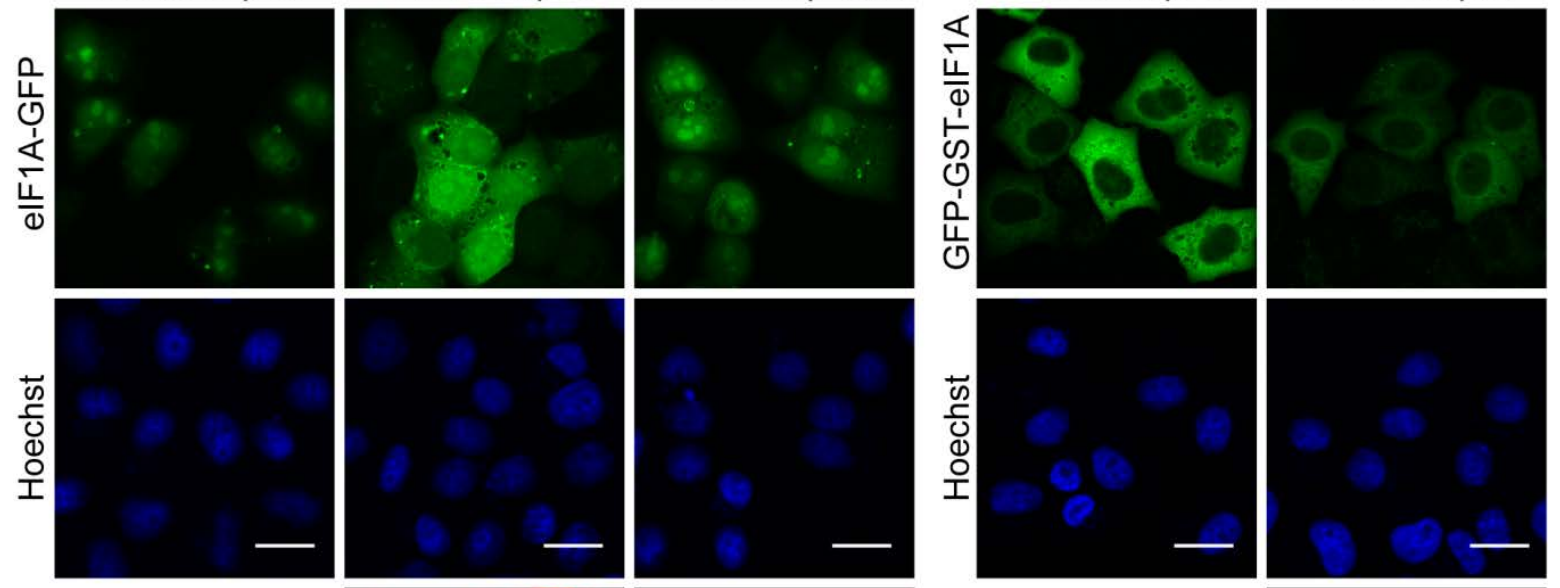

$\frac{m}{\frac{m}{2}}$
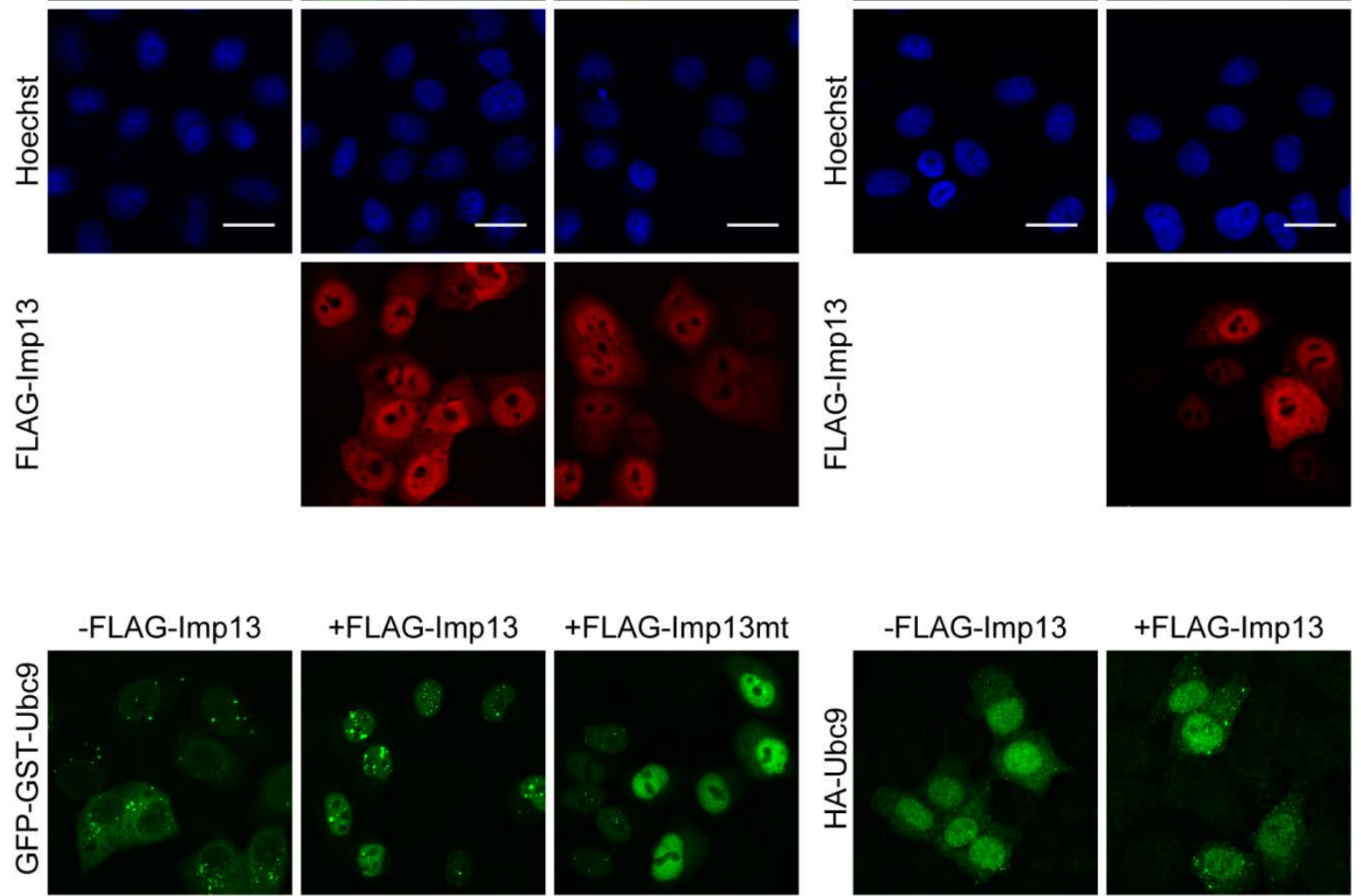

+FLAG-Imp13
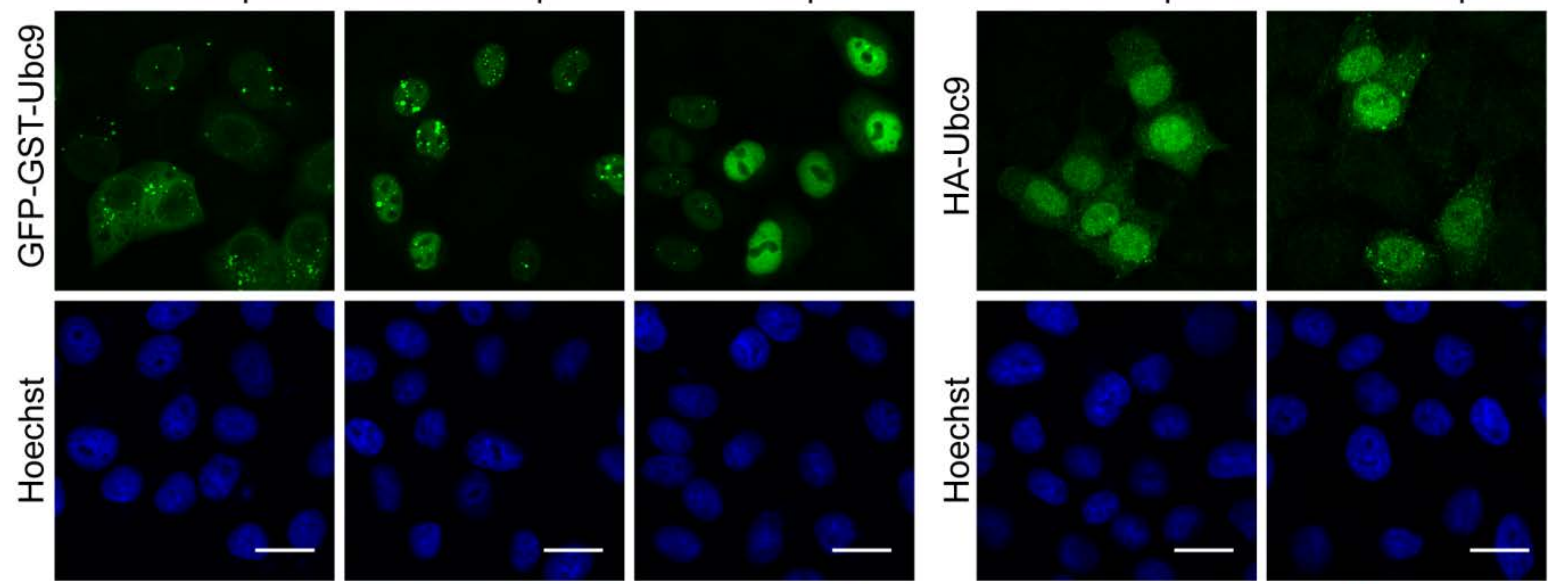

$\frac{m}{\frac{n}{E}}$
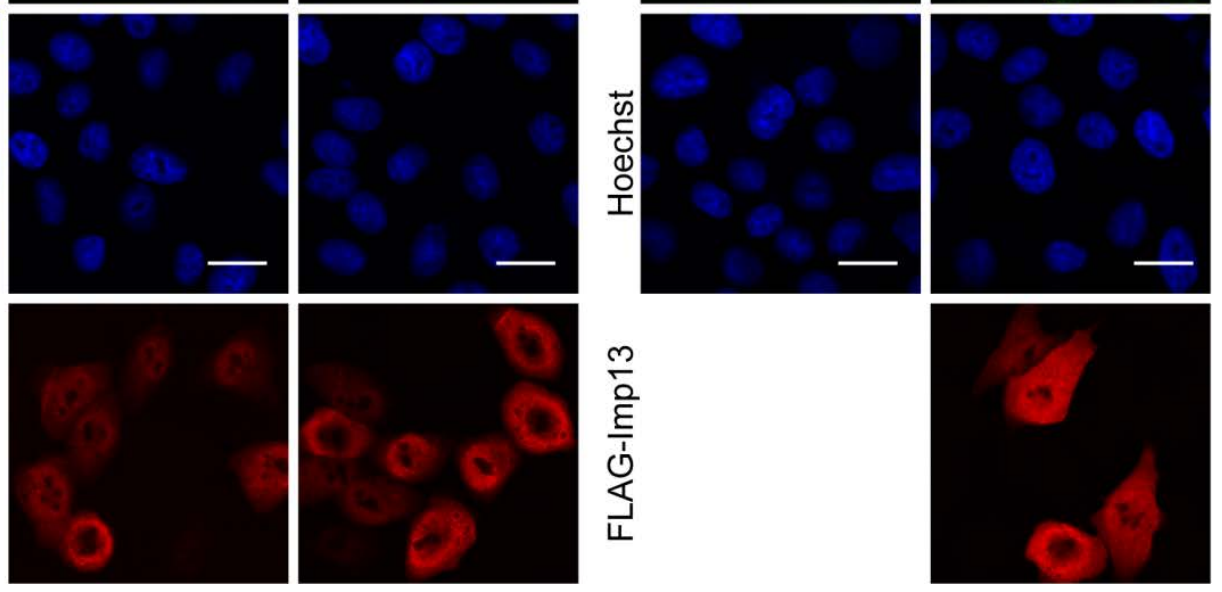

Figure 6: Importin 13 promotes nuclear import of Ubc9 and nuclear export of elF1A. HeLa P4 cells were transiently transfected using the calcium phosphate method with plasmids coding for Ubc9 or elF1A and importin 13 wild-type (+FLAG-Imp13), importin 13-E436R/D481R (+FLAG-Imp13mt; mutant impaired in elF1A export) or an empty control vector. HA-Ubc9 and FLAG-importin 13 were visualized by indirect immunofluorescence with an anti-HA and anti-FLAG antibody, respectively. The scale bars correspond to $20 \mu \mathrm{m}$. 
In summary, importin 13 can promote the import and export of overexpressed GFP-GST-Ubc9 and elF1A-GFP, respectively, while the importin 13 mutant Glu436Arg/Asp481Arg cannot export elF1A but is still capable of importing Ubc9. Consequently, eIF1A-GFP and GFP-GST-Ubc9 are effective controls for importin 13 mediated transport in importin 13 overexpression experiments.

\subsubsection{Importin 13 Mediates Nuclear Import of Ubc9 In Vitro}

Mingot et al., 2001 (135) showed that fluorescently labeled GST-Ubc9 is specifically imported by importin 13 into the nuclei of digitonin permeabilized HeLa cells and that the import efficiency is stimulated in the presence of Ran and an energy-regenerating system. To test whether these findings can be reproduced and to ascertain that the recombinant purified GST-Ubc9 and His-importin 13 are functional, transport assays were performed. HeLa P4 cells were permeabilized with digitonin, which selectively permeabilizes the plasma membrane but leaves the nuclear envelope intact, washed to remove soluble transport factors and incubated with defined transport mixes to analyze the import of GST-Ubc9 (Figure 7).

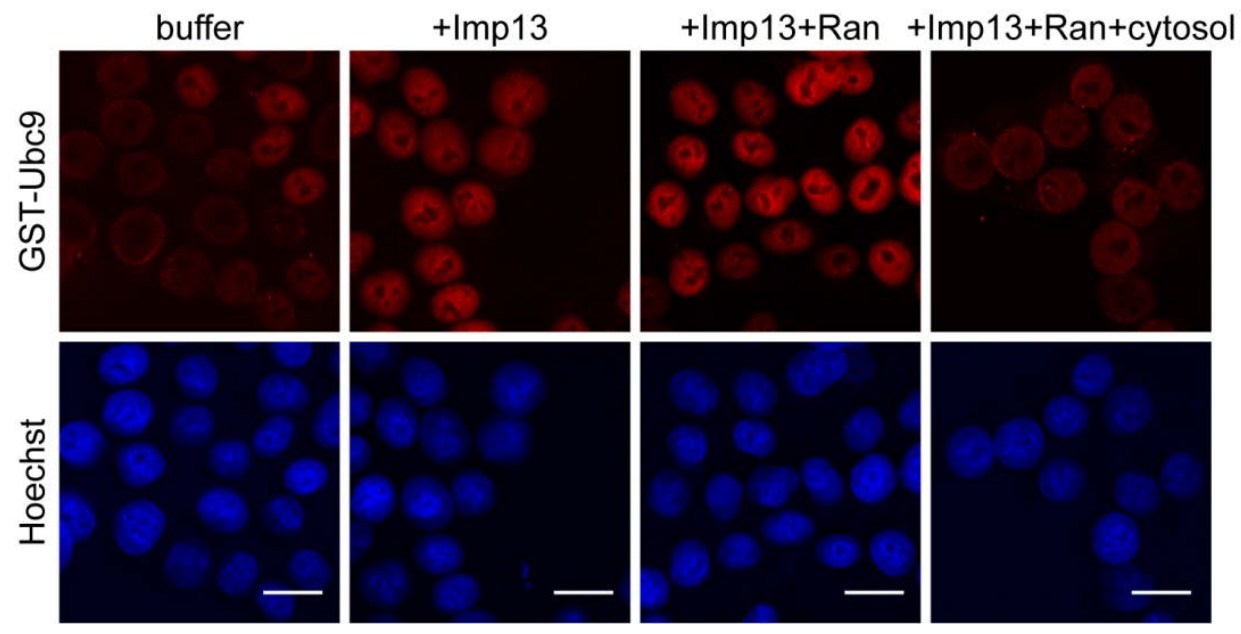

Figure 7: Importin 13 mediates import of GST-Ubc9. Digitonin permeabilized HeLa P4 cells were incubated with GST-Ubc9, an energy-regenerating system and buffer or His-importin 13 in the absence or presence of Ran and cytosol, as indicated. GST-Ubc9 was visualized by indirect immunofluorescence with an anti-GST antibody. The detector gain was decreased for GST-Ubc9+Imp13+Ran from 666 to 621 as the signal intensity was too strong. The scale bars correspond to $20 \mu \mathrm{m}$. 
In the absence of importin 13, only a minimal import of GST-Ubc9 was detected. Addition of importin 13 resulted in a clear accumulation of GST-Ubc9 in the cell nuclei and import was further enhanced in the presence of Ran, confirming the results from Mingot et al., 2001 (135). Interestingly, addition of cytosol strongly reduced nuclear import of GST-Ubc9, suggesting that some factor present in the cytosol prevents nuclear localization of Ubc9, either by inhibiting import or by promoting export of Ubc9. These results confirm that Ubc9 is imported into cell nuclei by importin 13 and that import is enhanced in the presence of Ran.

\subsubsection{Importin 13 Directly Interacts with Ubc9}

Mingot et al., 2001 (135) not only demonstrated that nuclear import of Ubc9 depends on importin 13 but also showed that Ubc9 as well as the export cargo elF1A directly interact with importin 13 in a RanGTP-dependent manner. As ultimately binding assays are to be used for the identification of importin 13 substrates, it was tested if the reported results are reproducible and whether our recombinantly purified proteins are fully functional. GST-tagged Ubc9 and elF1A were immobilized on beads and incubated with either His- or Hzz (His- and zz-tag)-tagged importin 13 in the absence or presence of Ran Q69L loaded with GTP (Figure 8A). RanGTP ${ }_{\mathrm{Q} 69 \mathrm{~L}}$ is a mutant deficient in hydrolysis of Ran bound GTP and therefore is predominantly found in the GTP-bound form (186). This Ran mutant promotes binding of export cargoes to their nuclear transport receptor and strongly inhibits binding of import cargoes. As expected both His-importin 13 and Hzz-importin 13 bound efficiently to the import cargo GST-Ubc9 and binding was significantly reduced in the presence of RanGTP ${ }_{Q 69 L}$. Equimolar amounts of RanGTP ${ }_{Q 69 L}$ were not sufficient to completely abolish importin 13 binding to GST-Ubc9, suggesting that an excess of RanGTP is required to fully prevent the formation of importin 13 import complexes. In comparison to GST-Ubc9, binding of the export cargo GST-eIF1A was much less efficient and was dependent on the presence of RanGTP ${ }_{\mathrm{Q} 69 \mathrm{~L}}$. Only binding of Hzz-importin 13 but not His-importin 13 to GST-eIF1A could be observed. 


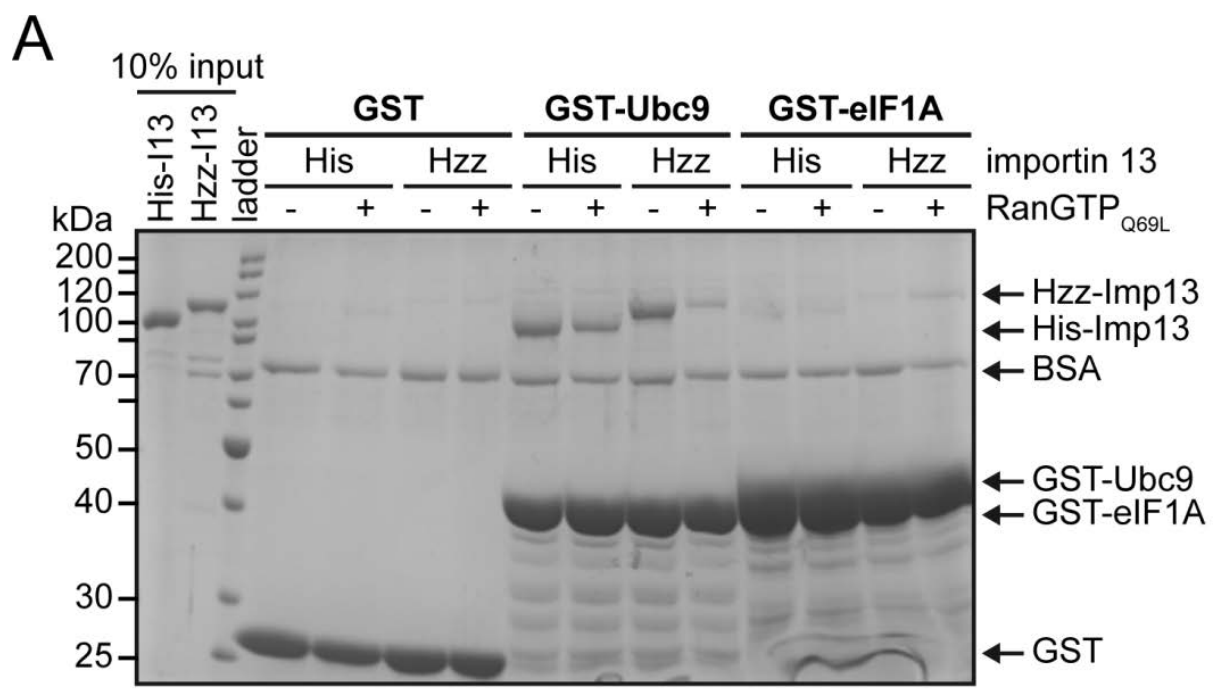

B

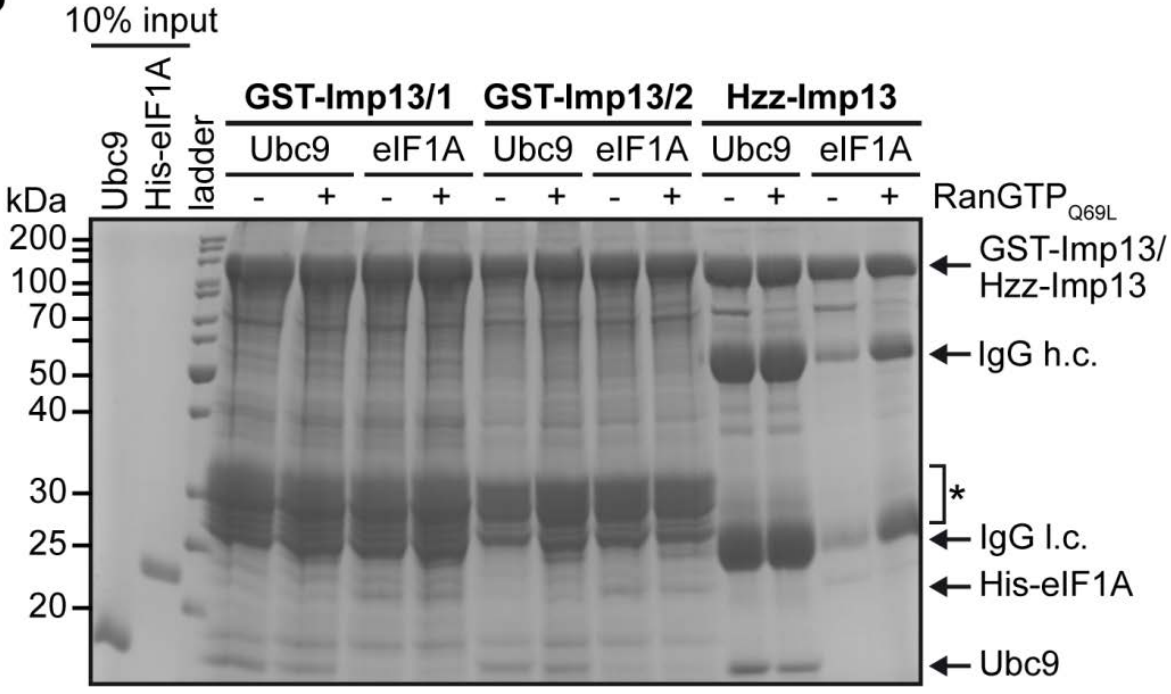

Figure 8: Importin 13 directly interacts with Ubc9 and elF1A. In (A) GST-tagged Ubc9 and elF1A $(20 \mu \mathrm{g})$ were immobilized on glutathione-Sepharose beads and incubated with $5 \mu \mathrm{g}$ His- or Hzz-tagged importin 13. In (B) GST- and Hzz-tagged importin $13(20 \mu \mathrm{g})$ was immobilized on glutathione-Sepharose and IgG-Sepharose, respectively, and incubated with $5 \mu \mathrm{g}$ untagged Ubc9 or $7 \mu \mathrm{g}$ His-elF1A. All reactions were performed in the absence or presence of $5 \mu \mathrm{g} \mathrm{RanGTP} \mathrm{Q}_{\mathrm{Q} 9 \mathrm{~L}}$ in PBS supplemented with $2 \mathrm{mg} / \mathrm{mL}$ BSA. Proteins bound to GST-tagged proteins were eluted with $4 \mathrm{x}$ SDS-sample buffer and proteins bound to Hzz-importin 13 were eluted by glycine elution. Interacting proteins were analyzed by SDS-PAGE, followed by Coomassie staining. Asterisk in (B) marks contaminants visible between $25-35 \mathrm{kDa}$ that likely correspond to GST-importin 13 degradation products. GST-importin 13/1: expressed from plasmid \#1576 with a linker of 13 amino acids; GST-importin 13/2: expressed from plasmid \#887 with a linker of 17 amino acids; I.c.: light chain; h.c.: heavy chain; ladder: PageRuler Unstained Protein Ladder. 
To test whether the cargoes can also bind to immobilized importin 13, reverse binding experiments were performed using three differently tagged importin 13 proteins. Binding experiments were done with two GST-importin 13 variants that differed in the size of their linker and one Hzz-tagged importin 13 variant. The Hzz-tag consists of a His-tag and two IgG-binding domains of protein A from Staphylococcus aureus (zz-tag). The three importin 13 variants were immobilized on beads and incubated with untagged Ubc9 or His-elF1A in the absence or presence of RanGTP ${ }_{\mathrm{Q} 69 \mathrm{~L}}$ (Figure 8B). Both Ubc9 and HiselF1A bound efficiently to all three importin 13 fusion proteins in a RanGTP-dependent manner, with Ubc9 showing a slightly higher affinity for Hzz-importin 13 and His-elF1A showing a slightly higher affinity for GST-importin 13. As in the previous binding experiment, binding of Ubc9 to importin 13 was stronger than for His-elF1A. The identity of the bound proteins was confirmed by immunoblotting with an anti-Ubc9 and anti-elF1A antibody (data not shown).

In summary, the reported direct interaction between importin 13 and its cargoes Ubc9 and elF1A could be confirmed using proteins with various tags. Binding of importin 13 cargoes to the two GST-importin 13 variants was comparable but in the case of Ubc9 less efficient than binding to Hzz-importin 13. Further, GST-importin 13 was more prone to degradation than Hzz-importin 13. Thus, Hzz-importin 13 was used in subsequent binding experiments and ultimately also in the pull-down experiment coupled to mass spectrometry for the identification of importin 13 substrates.

\subsubsection{Importin 13 Binds Endogenous Ubc9 from HeLa P4 Cell Extracts}

One central aim of this study was to identify importin 13 cargoes that bind to immobilized importin 13 from a HeLa P4 cell extract. Previously, binding of endogenous Ubc9 and elF1A from a HeLa lysate to immobilized importin 13 has been demonstrated by mass spectrometry (135). Binding conditions were established with immobilized importin 13 using the known importin 13 cargoes Ubc9 and elF1A. Hzz-tagged importin 13 was immobilized on beads and incubated with a digitonin or freeze/thaw HeLa P4 lysate in the

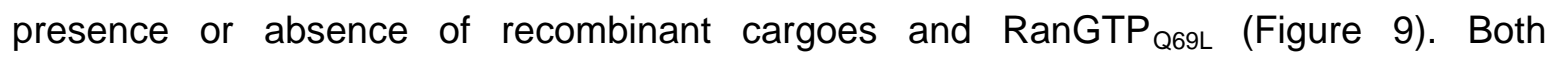
endogenous and recombinant Ubc9 bound efficiently to Hzz-importin 13 from both HeLa P4 cell extracts with slightly more Ubc9 binding from the digitonin HeLa P4 cell lysate. Ubc9 binding was not affected by equimolar amounts of RanGTP ${ }_{\mathrm{Q} 69 \mathrm{~L}}$ showing that RanGTP becomes limiting in the presence of cell lysate, which contains high concentrations of other nuclear transport receptors that compete for RanGTP binding. 


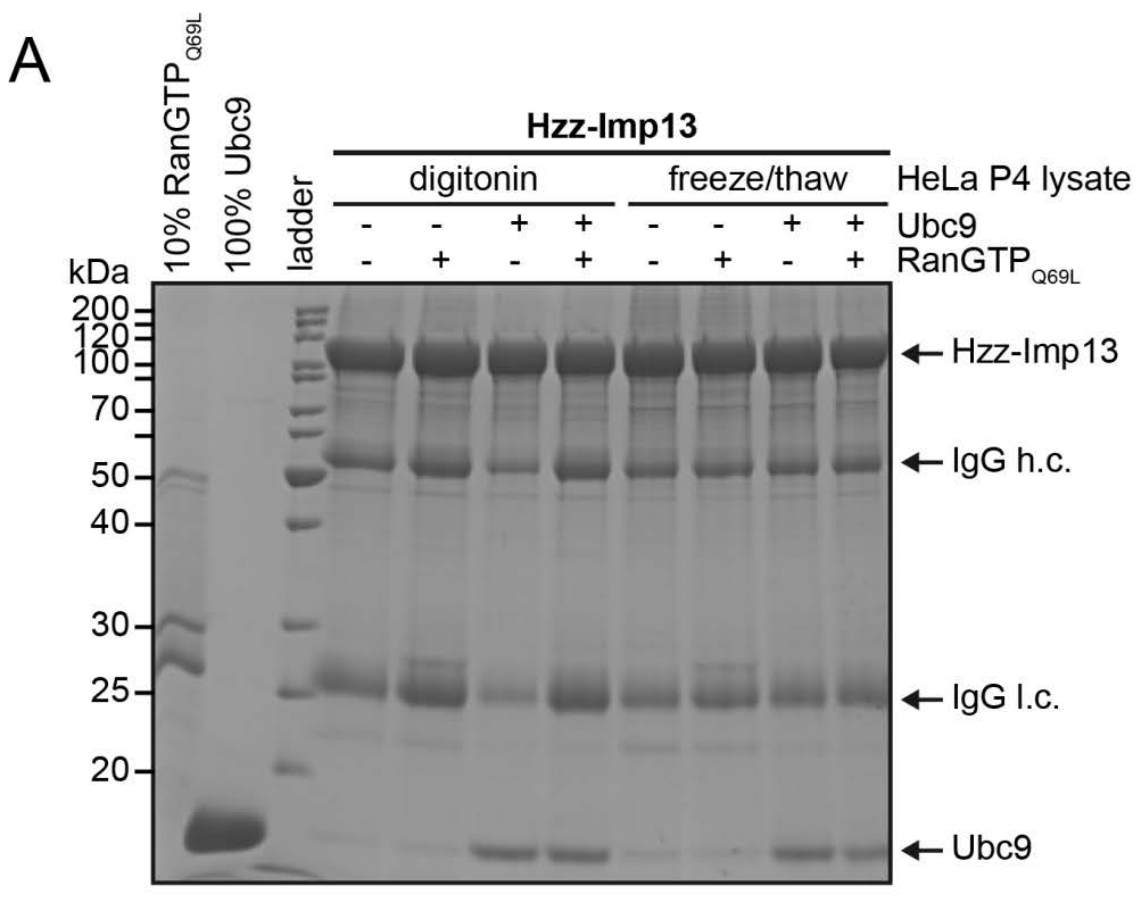

B

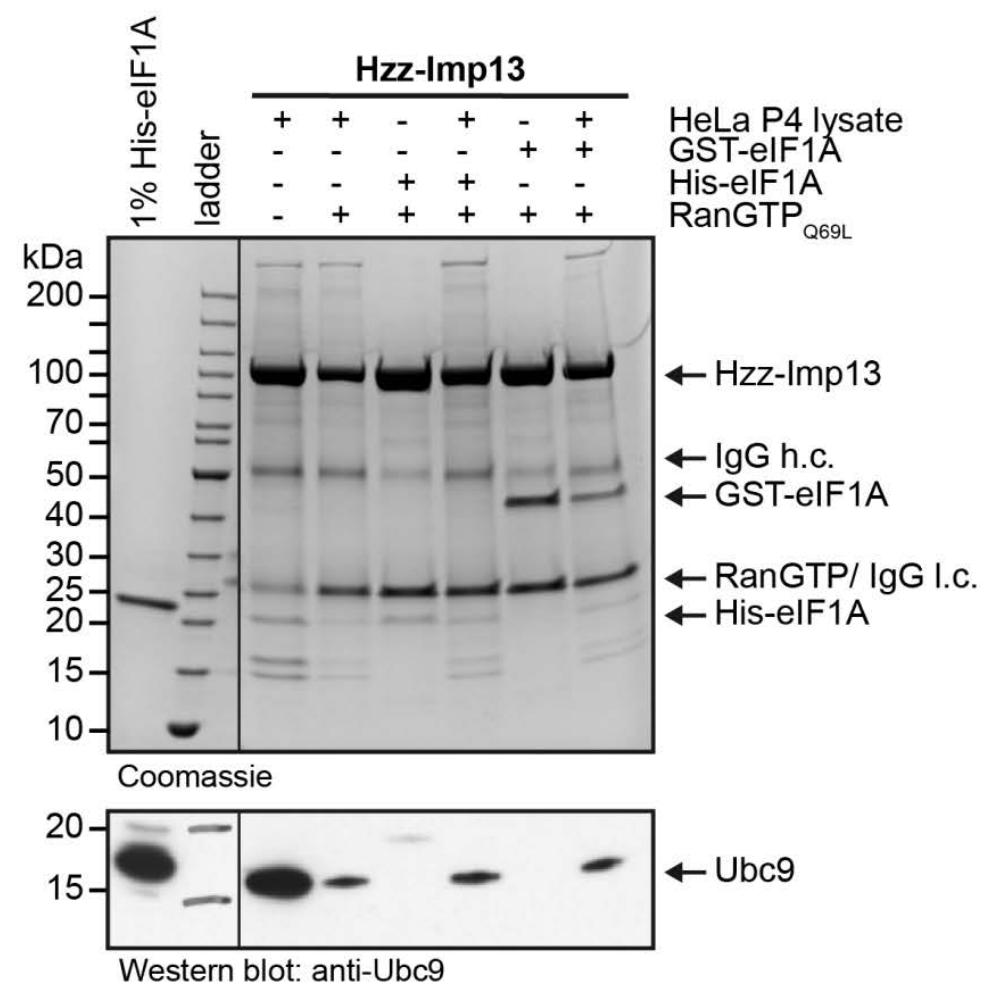

Figure 9: Importin 13 binds endogenous Ubc9 and recombinant elF1A from HeLa P4 cell extract. Hzz-importin $13(20 \mu \mathrm{g})$ was immobilized on IgG-Sepharose and incubated with a digitonin or freeze/thaw HeLa P4 cell extract in the absence or presence of (A) $5 \mu \mathrm{g}$ Ubc9 or $5 \mu \mathrm{g}$ RanGTP $_{\text {Q69L }}$ and (B) $7 \mu \mathrm{g}$ His-elF1A, $7 \mu \mathrm{g} \mathrm{GST-elF1A} \mathrm{or} 15 \mu \mathrm{g}$ RanGTP $_{\mathrm{Q} 69 \mathrm{~L}}$ in PBS supplemented with $2 \mathrm{mg} / \mathrm{mL}$ BSA. Bound proteins were eluted and analyzed as in (Figure 8). In (B) a freeze/thaw 
HeLa P4 cell lysate was used and binding of endogenous Ubc9 was detected by immunoblotting with an anti-Ubc9 antibody. I.c.: light chain; h.c.: heavy chain; ladder: PageRuler Unstained Protein Ladder.

For the export cargo elF1A, only binding of recombinant His-eIF1A and GST-elF1A but not endogenous elF1A to Hzz-importin 13 could be detected (Figure 9), which was further confirmed by immunoblotting (data not shown). Binding of recombinant GST-elF1A to Hzzimportin 13 was more efficient than binding of His-elF1A and both interactions were significantly reduced in the presence of HeLa P4 cell extract suggesting competition of other cargoes with elF1A for importin 13 binding. Indeed, concomitant binding of Ubc9 could be confirmed by immunoblot analysis in binding reactions performed with HeLa P4 cell extract. The immunoblot also showed that binding of endogenous Ubc9 was reduced if exogenous RanGTP $\mathrm{Q}_{6 \mathrm{~L}}$ was present in the binding reaction.

An interaction between importin 13 and Ubc9 or elF1A could further be confirmed using in situ proximity ligation assays (Figure S1). Endogenous proteins were targeted with antibodies and in situ interactions were visualized as single fluorescent dots using the proximity ligation assay. Knock-down of importin 13 reduced the number of fluorescent dots (one fluorescent dot corresponds to one single protein-protein interaction), confirming the specificity of the observed interactions. Interestingly, in situ interactions of importin 13 with Ubc9 were mainly observed in the nucleus, while interactions of importin 13 with elF1A were mainly observed in the cytoplasm. This suggests that the importin 13 import complexes are more transient in the cytoplasm than in the nucleus and vice versa for export complexes.

The results demonstrate that importin 13 binding experiments with HeLa P4 cell lysate could be an effective approach to identify new importin 13 cargoes. However, the results also show that the efficiency of cargo identification will depend strongly on the affinity of importin 13 for its cargoes. As demonstrated above, Ubc9, which has a reported eightfold lower dissociation constant than elF1A (107), also showed stronger binding to importin 13 than elF1A. Furthermore, binding of endogenous Ubc9 to Hzz-importin 13 was found to be slightly more efficient from a HeLa P4 cell extract generated by digitonin treatment, suggesting that a digitonin cell lysate might be more effective for the identification of importin 13 substrates than a freeze/thaw cell lysate. 


\subsection{Identification of Potential Importin 13 Export Cargoes Using an Importin 13 Overexpression Screen}

\subsubsection{Importin 13 Overexpression Screen Using a Library of Nuclear Proteins}

When this thesis was started several importin 13 import cargoes had been identified but only one export cargo, the translation initiation factor elF1A was known. To specifically screen for potential importin 13 export cargoes, labeled proteins from a library of proteins with known nuclear localization were transiently expressed in HeLa P4 cells and monitored for any changes in subcellular localization upon coexpression of importin 13. The plasmid clones coding for the tagged nuclear proteins were derived from the 'LIFE database' (174, 175).

The initial overexpression screen to identify potential importin 13 export cargoes was performed by Annegret Nath, a former member of our group (data not shown). While the majority of $\sim 200$ tested proteins was not affected (>60\%), several proteins changed their subcellular localization upon importin 13 coexpression. Clear effects were observed for about $10-15 \%$ of the proteins.

Candidate proteins that were affected as well as proteins that were not affected by importin 13 overexpression in the initial screen were reanalyzed for effects of importin 13 coexpression on their subcellular localization (Table S1). As in the initial overexpression screen, plasmids coding for tagged proteins were transiently transfected into HeLa P4 cells and changes in subcellular distribution upon coexpression of importin 13 were analyzed (Figure 10 and Figure S2). The known importin 13 cargoes elF1A-GFP and GFP-GST-Ubc9 were included in the screen as positive controls and both confirmed that the coexpressed FLAG-importin 13 was fully functional. Additionally, several negative controls were included such as different fusion tags, known artificial cargoes of other nuclear transport receptors as well as randomly selected proteins. 


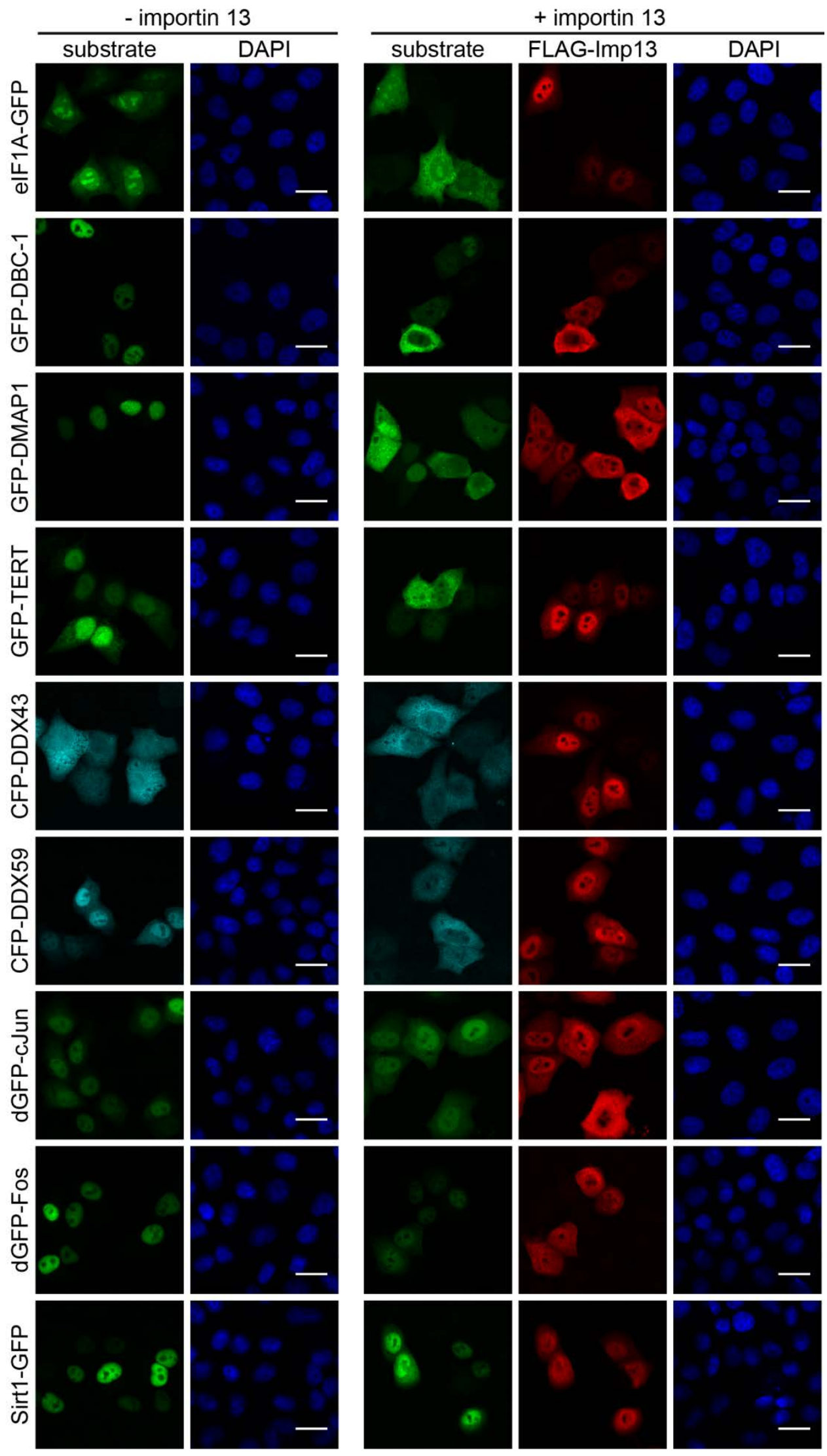


Figure 10: Importin 13 affects the subcellular localization of DBC-1, DMAP1, TERT, DDX43, DDX59, cJun, Fos and Sirt1. HeLa P4 cells were transiently cotransfected with plasmids coding for fluorescently tagged DBC-1, DMAP1, TERT, DDX43, DDX59, cJun, Fos or Sirt1 and FLAGimportin 13 or an empty control vector using the calcium phosphate method. FLAG-importin 13 was visualized by indirect immunofluorescence with an anti-FLAG antibody. For controls and further proteins tested see supplemental Figure S2 and Table S1. The scale bars correspond to $20 \mu \mathrm{m}$.

Similar to the findings of the initial screen, coexpression of importin 13 had strong effects on the nuclear proteins DBC-1, DMAP1, TERT, DDX43, DDX59 and c-Jun, promoting their cytoplasmic localization (see Table S1 for full protein names). The effect of importin 13 on DBC-1 and C-Jun is not entirely surprising, as both proteins have previously been reported to bind to importin $13(49,117)$. However, the binding was reduced in the presence of RanGTP $_{\mathrm{Q} 69 \mathrm{~L}}$, implicating them rather as importin 13 import than export cargoes $(49,117)$. Proteins that were slightly affected by importin 13 overexpression included Fos, Tmp29, Nip30 and Sirt1. Surprisingly, the M9 nuclear import signal (PY-NLS found in hnRNPA1 and A2), which is recognized by transportin, was also slightly affected by importin 13 overexpression, resulting in a more cytoplasmic localization (Figure S2).

The other artificial cargoes, including dGFP-GST-cNLS (Xenopus), which is imported by importin $\alpha / \beta$ and dGFP-GST-RevNLS (from HIV-1 Rev), which is imported by transportin and potentially several other karyopherins $(48,116)$, as well as the Crm1 cargo protein snurportin 1 (187), were not affected by importin 13 coexpression. These GFP-fusion constructs also demonstrate that the importin 13 mediated redistribution of the identified proteins is not an unspecific effect of the GFP-tag. However, to fully exclude that the tag might affect the subcellular localization, other fusion tags for both importin 13 and the potential importin 13 cargoes were also tested (Figure S2 or data not shown). Irrespective of the tag used, importin 13 coexpression always resulted in a shift towards the cytoplasm of DBC-1, DMAP1, TERT, DDX43 and DDX59. However, with all HA-tagged constructs the observed effects were less strong.

For yet unidentified reasons, the importin 13 effect varied not only between different experiments but also between individual cells, with some cells showing a strong redistribution of DBC-1 and DMAP1 and others only a weak or even no redistribution upon importin 13 coexpression. Although a trend could be observed that cells with high importin 13 expression levels had a more cytoplasmic localization of DBC-1 and DMAP1, the same could also be observed in cells with low importin 13 expression levels, albeit less frequently. Interestingly, importin 13 induced relocalization of DBC-1 and DMAP1 was 
strongest in cells sub-cultivated for only one to three passages after thawing from liquid nitrogen (data not shown). This suggests that HeLa P4 cells thawed from liquid nitrogen take longer to recover or that a stress response is induced in these cells that affects importin 13 nucleocytoplasmic transport. However, heat shock did not influence the observed importin 13 effect (data not shown). The importin 13 mediated relocalization also is not cell specific, as similar effects were also observed in 293T HEK, U2OS and COS-7 cells (data not shown). Interestingly, with some of the identified proteins not only the candidate protein but also importin 13 showed relocalization to the cytoplasm and a very similar distribution pattern, suggesting that they form stable complexes in the cytoplasm. In summary, DBC-1, DMAP1, TERT, DDX43, DDX59 and c-Jun were significantly affected by importin 13 overexpression, while the effect on Fos, Tmp29, Nip30 and Sirt1 was less strong. Even though these proteins showed a clear shift towards the cytoplasm upon importin 13 overexpression, one cannot conclude with certainty that these proteins are importin 13 export cargoes. Importin 13 could also indirectly enhance the cytoplasmic localization of these proteins or function as a negative regulator of nuclear import.

\subsubsection{DBC-1, DMAP1, DDX43 and DDX59 Bind Importin 13 Differently to eIF1A}

The importin 13 mutant Glu436Arg/Asp481Arg is deficient in elF1A export (107). To test whether the putative importin 13 export cargoes DBC-1, DMAP1, DDX43 and DDX59 bind to the same key residues on importin 13 as elF1A, plasmids coding for these cargoes were transfected in HeLa P4 cells and the effect of FLAG-importin 13-E436R/D481R cotransfection was analyzed (Figure 11). While elF1A only changed its subcellular localization upon coexpression of wild type but not mutant importin 13, the putative importin 13 export cargoes showed relocalization to the cytoplasm with both wild type and mutant importin 13. Thus, these proteins either have a different binding site on importin 13 or more residues need to be mutated to abolish the interaction. As in the previous experiment (3.2.1), importin 13 showed a more cytoplasmic localization in some cells if coexpressed with DBC-1 or DMAP1.

The results indicate that DBC-1, DMAP1, DDX43 and DDX59 bind importin 13 differently than the established export cargo elF1A. 

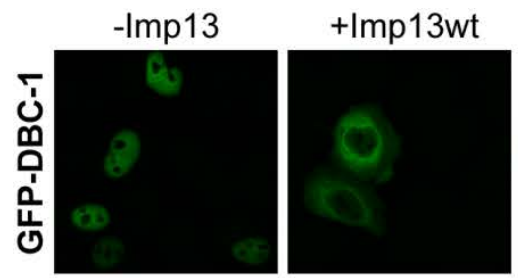

+Imp13mt

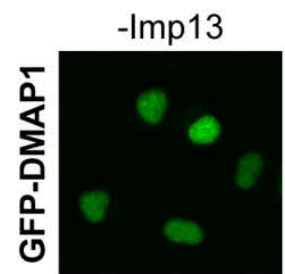

+Imp13wt

+Imp13mt
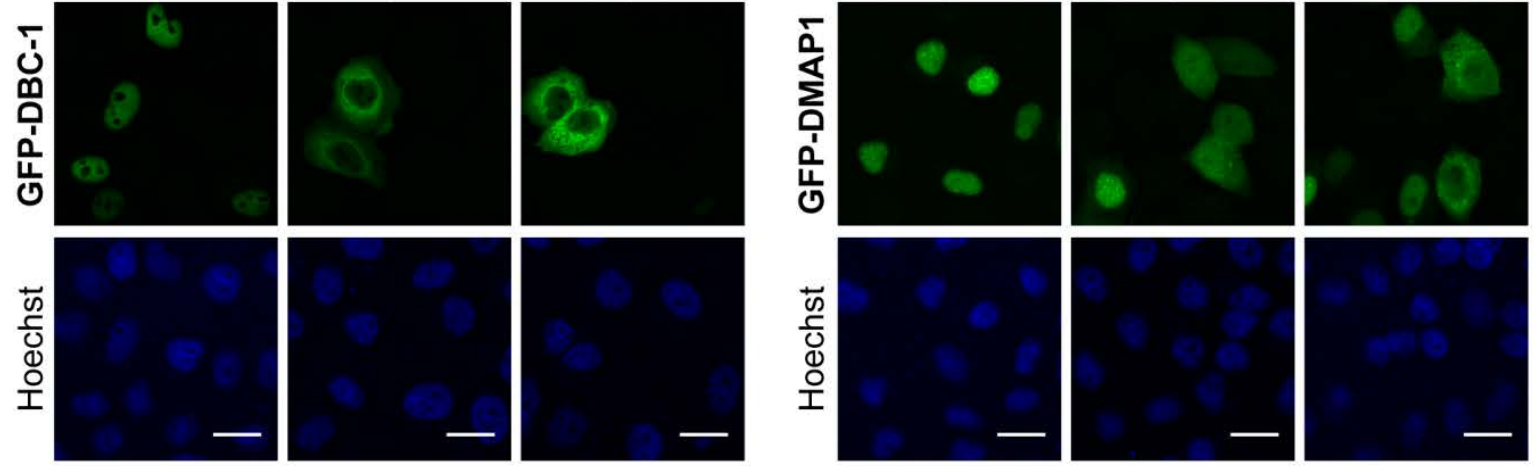

高
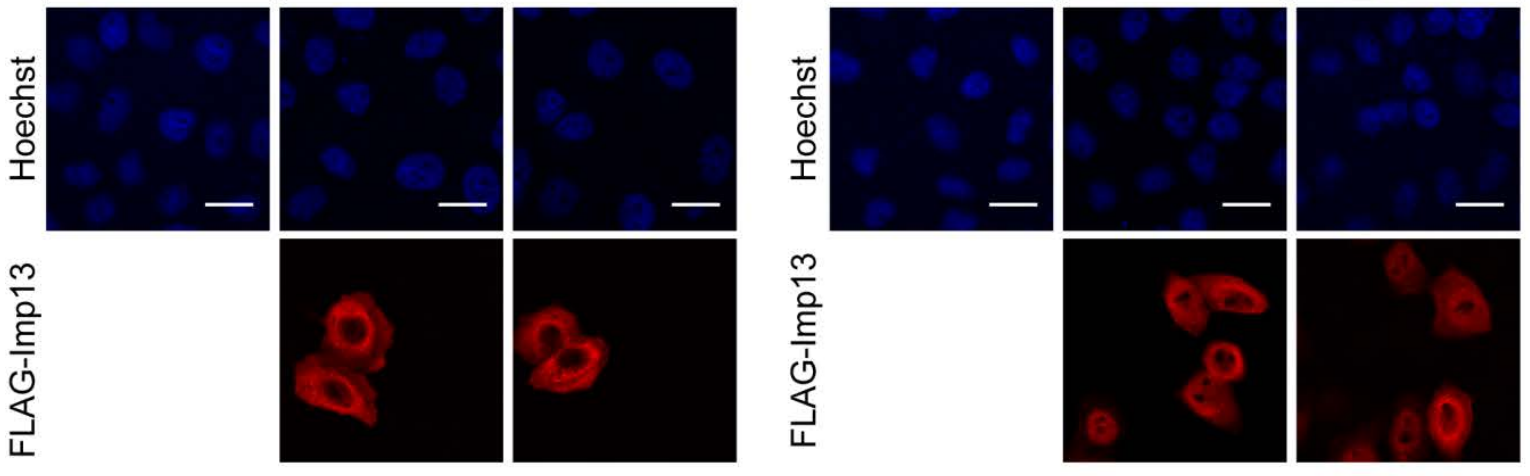

+ Imp13wt

+Imp13mt
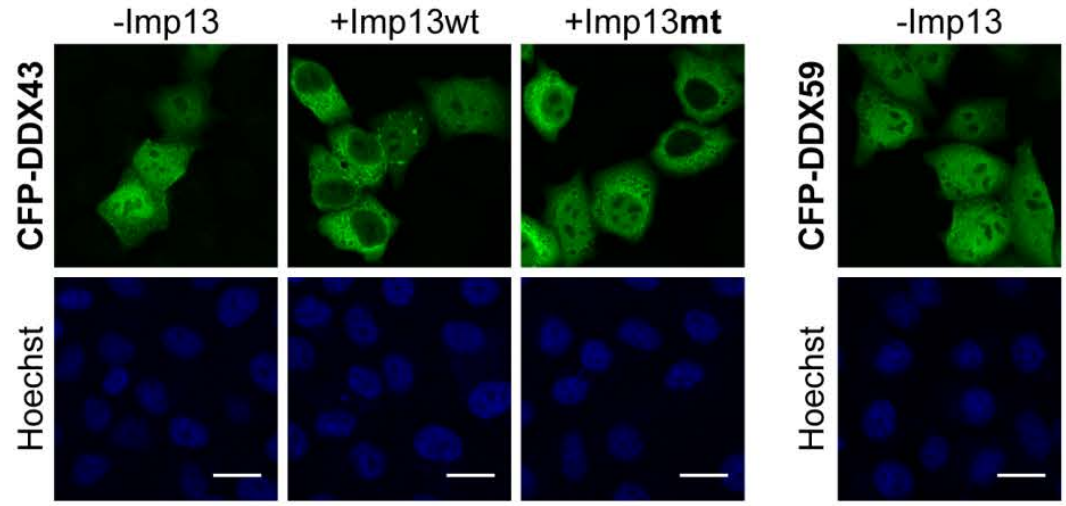

+ Imp13wt

+Imp13mt
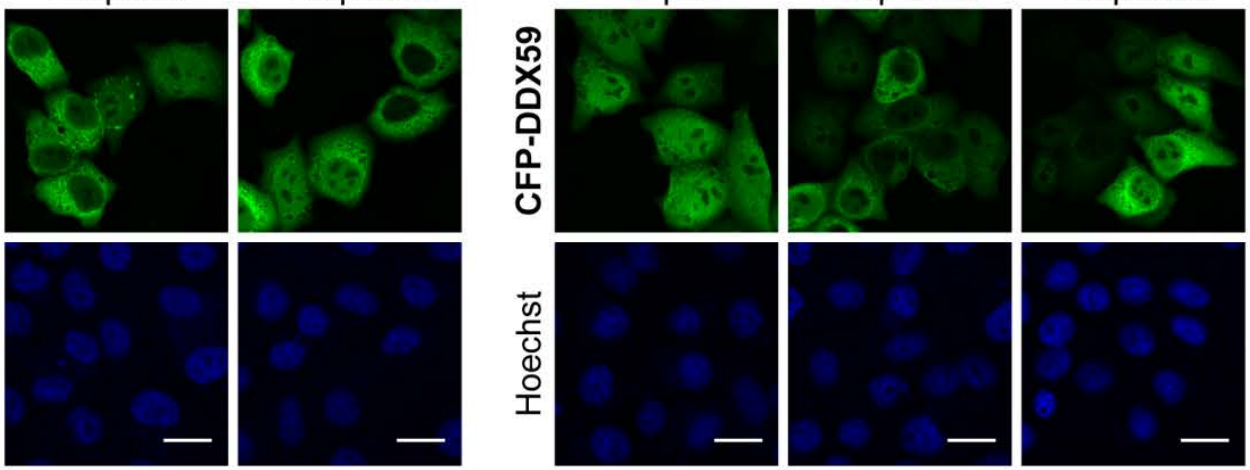

$\frac{m}{\frac{n}{E}}$
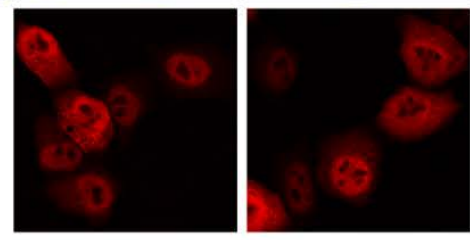

$\frac{m}{\frac{m}{\text { ह }}}$
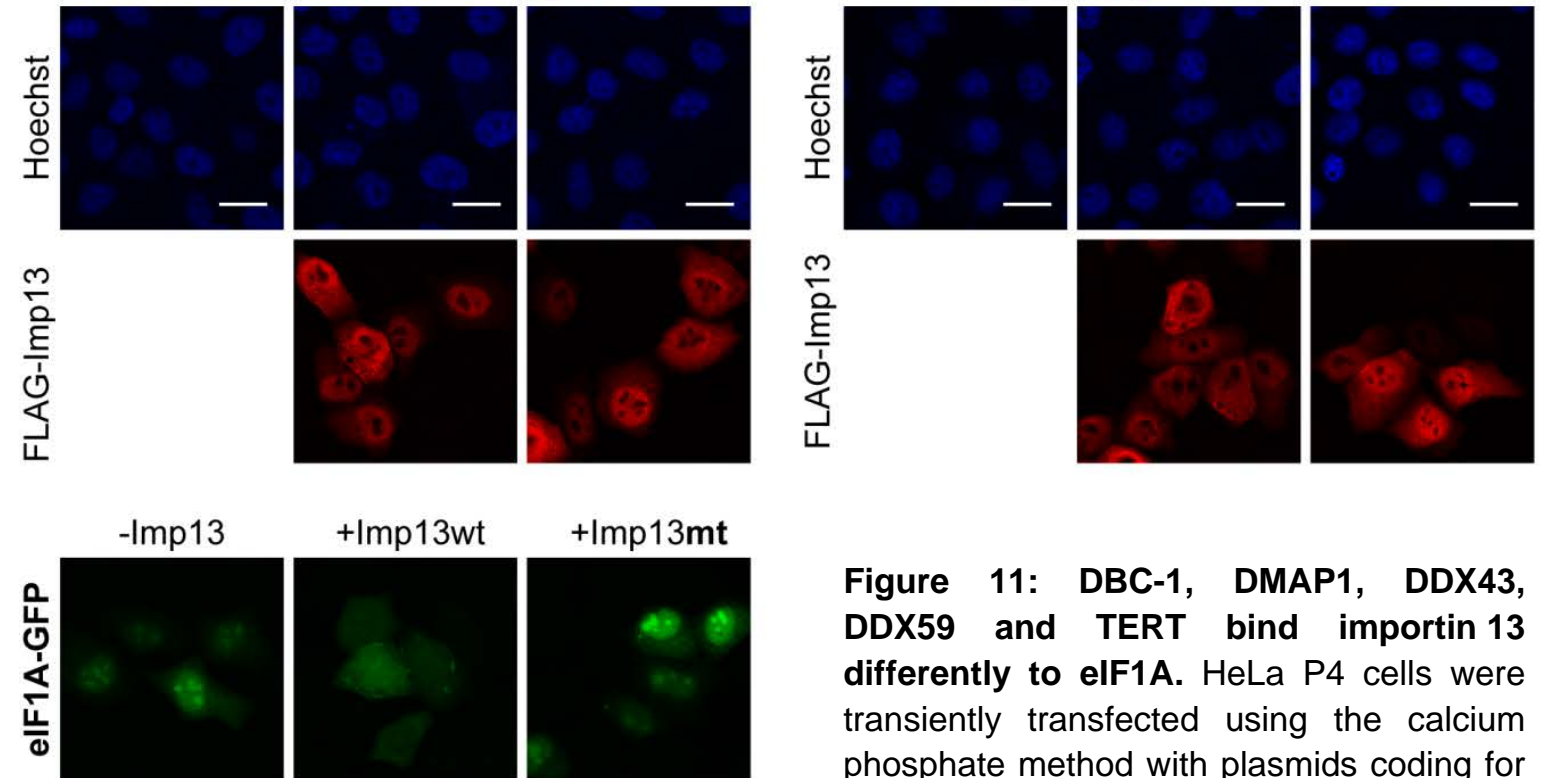

+ Imp13wt

+Imp13mt
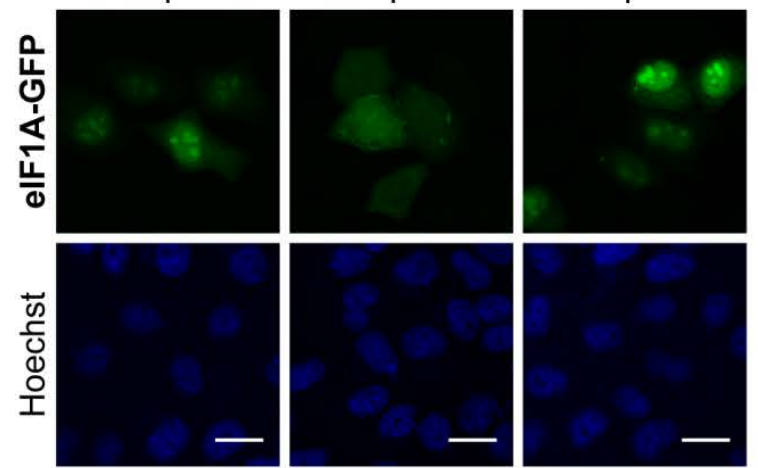

$\frac{m}{\frac{m}{\circ}} \frac{1}{1}$
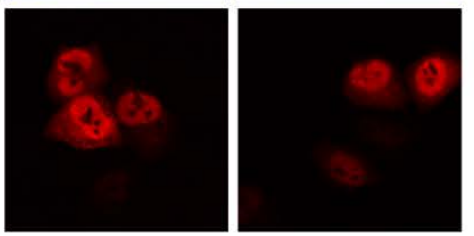

Figure 11: DBC-1, DMAP1, DDX43, DDX59 and TERT bind importin 13 differently to elF1A. HeLa P4 cells were transiently transfected using the calcium phosphate method with plasmids coding for GFP- or CFP-tagged DBC-1, DMAP1, DDX43, DDX59 or elF1A and optionally cotransfected with FLAG-importin 13 wild type (+Imp13wt) or FLAG-importin 13E436R/D481R (+Imp13mt; mutant impaired in elF1A export). Transfected FLAGimportin 13 was visualized by indirect immunofluorescence with an anti-FLAG antibody. The scale bars correspond to $20 \mu \mathrm{m}$. 


\subsubsection{Characterization of DBC-1 Interaction with Importin 13}

\subsubsection{Importin 13 Interacts with the Coiled-coil Domain of DBC-1}

Previous experiments in the lab suggested that the coiled-coil domain of DBC-1 (amino acids 793-923) is required for the importin 13 mediated cytoplasmic localization. To test whether this is indeed the case, HeLa P4 cells were transiently transfected with plasmids coding for the coiled coil domain of DBC-1 fused to a cNLS, to localize it to the nucleus, and analyzed for changes in nucleocytoplasmic distribution upon importin 13 coexpression (Figure 12). In the absence of importin 13, the coiled-coil domain of DBC-1 fused to a cNLS was mainly nuclear but in the presence of importin 13, a shift to the cytoplasm could be observed. As shown for full-length DBC-1, the importin 13 mutant impaired in elF1A binding (importin 13-E436R/D481R), was still capable of relocalizing the coiled-coil domain of DBC-1 to the cytoplasm. This confirms that the coiled-coil domain of DBC-1 is required for interaction with importin 13. However, binding assays will need to be performed to show that the interaction is direct rather than indirect.

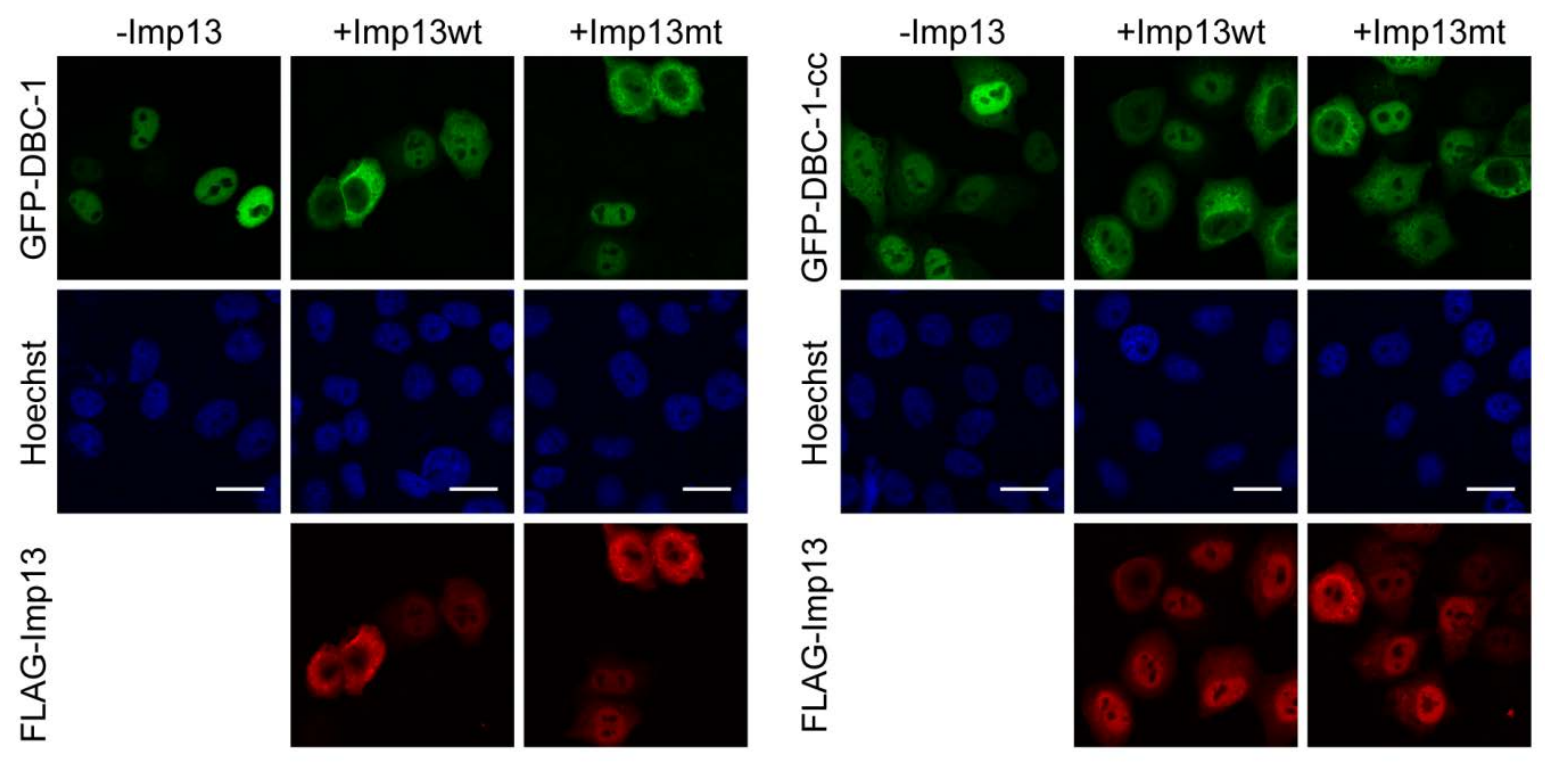

Figure 12: Importin 13 interacts with the coiled-coil domain of DBC-1. HeLa P4 cells were transiently transfected with plasmids coding for full length DBC-1 or the coiled-coil domain of DBC-1 (aa793-923, DBC-1-cc) using the calcium phosphate method. Optionally cells were cotransfected with plasmids coding for either wild type FLAG-importin 13 (+Imp13wt) or FLAG-importin 13E436R/D481R (+Imp13mt). FLAG-tagged importin 13 was visualized by indirect immunofluorescence using anti-FLAG antibody. The scale bars correspond to $20 \mu \mathrm{m}$. 


\subsubsection{The N-terminal Domain of Importin 13 Is Required for Recognition of DBC-1}

To get an idea as to where DBC-1 might bind to importin 13, the effect of different importin 13 fragments on the subcellular localization of DBC-1 was analyzed. A physiologically relevant $\mathrm{N}$-terminally truncated importin 13 isoform has previously been reported to function as a negative regulator of nuclear import in testis $(136,172)$, a function that could also be relevant for DBC-1. HeLa P4 cells were cotransfected with plasmids coding for GFP-DBC-1 and different N-terminal and C-terminal fragments of FLAG-tagged importin 13 (Figure 13).

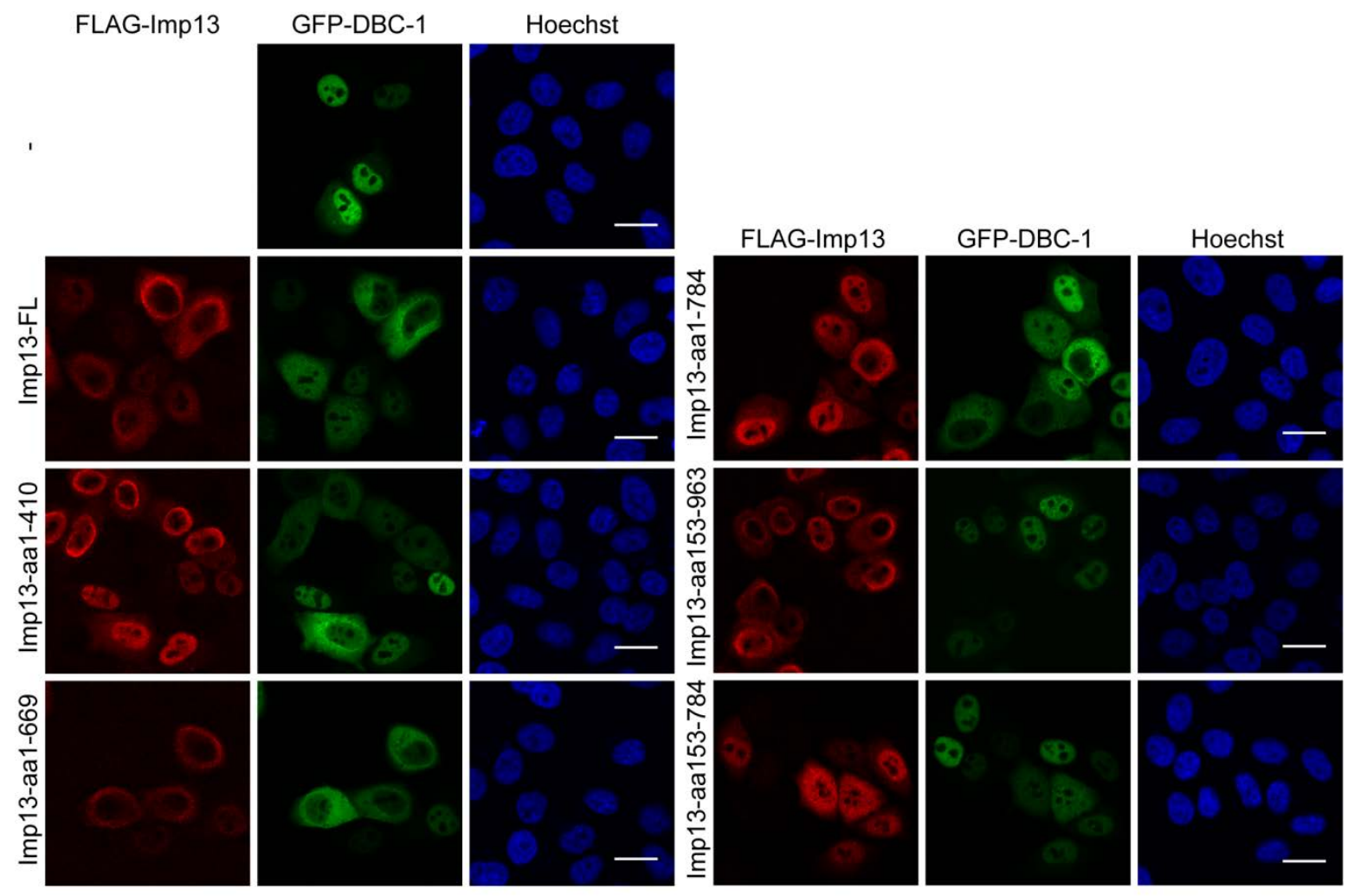

Figure 13: The C-terminus of importin 13 is dispensable for recognition of DBC-1. HeLa P4 cells were transiently transfected with plasmid DNA coding for GFP-DBC-1 and wild type or truncated FLAG-importin 13. The different FLAG-importin 13 constructs were visualized by indirect immunofluorescence with an anti-FLAG antibody. The scale bars correspond to $20 \mu \mathrm{m}$.

The C-terminal fragments of importin 13 resulted only in a minor relocalization of DBC-1 to the cytoplasm, whereas the $\mathrm{N}$-terminal fragments of importin 13 resulted in a clear shift towards the cytoplasm, similar to that seen with full-length importin 13. A weak shift 
towards the cytoplasm was detected with amino acids 1-410 of FLAG-tagged importin 13, while the strongest shift was detected with amino acids 1-669. This indicates that several binding sites on importin 13 contribute to DBC-1 binding and that the major binding sites are present on the $\mathrm{N}$-terminal arch of importin 13. However, binding to the C-terminal arch cannot be excluded, as the C-terminal fragments lack the N-terminal binding site for RanGTP, which is required for the formation of export complexes. Thus, even if binding would occur, the C-terminal importin 13 fragment would not be able to shuttle DBC-1 to the cytoplasm.

The other putative importin 13 export cargoes DMAP1, DDX43, DDX59 and TERT were also characterized for their interaction with importin 13 by cotransfection of different importin 13 fragments (data not shown). Similar to DBC-1, subcellular localization of these cargoes was not affected by C-terminal fragments but only by N-terminal fragments of importin 13. The longer the $\mathrm{N}$-terminal fragment, the stronger the observed redistribution to the cytoplasm. As for DBC-1 this indicates that at least a part of the importin $13 \mathrm{~N}$-terminus is involved in the interaction with these cargoes, while the far C-terminus appears to be dispensable.

\subsection{Identification of Potential Importin 13 Cargoes by Mass Spectrometry}

A major goal of this work was to significantly expand the number of known importin 13 cargoes. While the above importin 13 overexpression screen does allow for the identification of importin 13 interaction partners, it is limited in the number of available plasmids. Thus, two different mass spectrometry based approaches were established to allow for the large scale identification of importin 13 substrates. Both approaches focused on identifying proteins that bound to Hzz-importin 13 from a HeLa P4 cell lysate but differed in their strategy to reduce the number of false positive proteins. In the first approach, binding of proteins to both the Hzz-tag and Hzz-importin 13 from two different HeLa P4 cell extracts was compared, with the Hzz-tag reaction serving as a control for unspecific binding proteins. The second approach was based on quantitative mass spectrometry using stable isotope labeling with amino acids in cell culture (SILAC). To effectively distinguish between importin 13 import and export cargoes, binding reactions were performed in the absence or presence of Ubc9 and RanGTP $\mathrm{Q}_{\mathrm{Q} 9 \mathrm{~L}}$. RanGTP $\mathrm{R}_{\mathrm{Q} 6 \mathrm{~L}}$ is expected to promote the formation of importin 13 export complexes as it has been reported to displace importin 13 import cargoes and facilitate the binding of export cargoes to importin $13(93,135)$. In contrast, Ubc9 is expected to prevent the formation of both 
importin 13 import and export complexes as excess Ubc9 has been shown to compete with both RanGTP and other import cargoes for importin 13 binding $(93,135)$.

\subsubsection{Mass Spectrometry Based Identification of Potential Importin 13 Substrates}

To get an idea of the range of proteins that interact with importin 13, mass spectrometry analysis was performed to identify proteins that bind to immobilized Hzz-importin 13 from a HeLa P4 cell extract. Two different HeLa P4 cell extracts were compared, a freeze/thaw and a digitonin lysate to see whether cell lysate preparation affects the type of proteins identified. Digitonin is a reagent known to selectively permeabilize the plasma membrane and leave the nuclear envelope intact. In contrast, disruption by freeze/thaw is less selective potentially resulting in the release of harmful proteins from other cellular compartments such as proteases. To identify proteins that bind unspecifically to the Hzz-tag and the IgG-Sepharose, binding experiments were performed in parallel to Hzz-importin 13 with the Hzz-tag alone.

The Hzz-tag and Hzz-tagged importin 13 were immobilized on IgG-Sepharose and incubated with digitonin or freeze/thaw HeLa P4 cell extract. Bound proteins were eluted by magnesium chloride elution to prevent extensive co-elution of the IgG light and heavy chain. To assess the effectiveness of magnesium chloride elution, a second elution step was done with $4 \mathrm{x}$ SDS-sample buffer. Bound proteins eluted with magnesium chloride were separated by SDS-PAGE and protein peptides were extracted by in-gel tryptic digestion. Peptides were purified over C18 stage tips and given to our collaborators for mass spectrometry analysis. Raw data was analyzed with the Proteome Discoverer against a human protein database. Additionally, the magnesium chloride eluates were separated by SDS-PAGE and visualized by silver staining (Figure 14A). The silver stained gel showed that a lot of proteins bound unspecifically to the Hzz-tag and the IgG-Sepharose. However, more proteins were enriched with Hzz-importin 13 than with the Hzz-tag. 
A

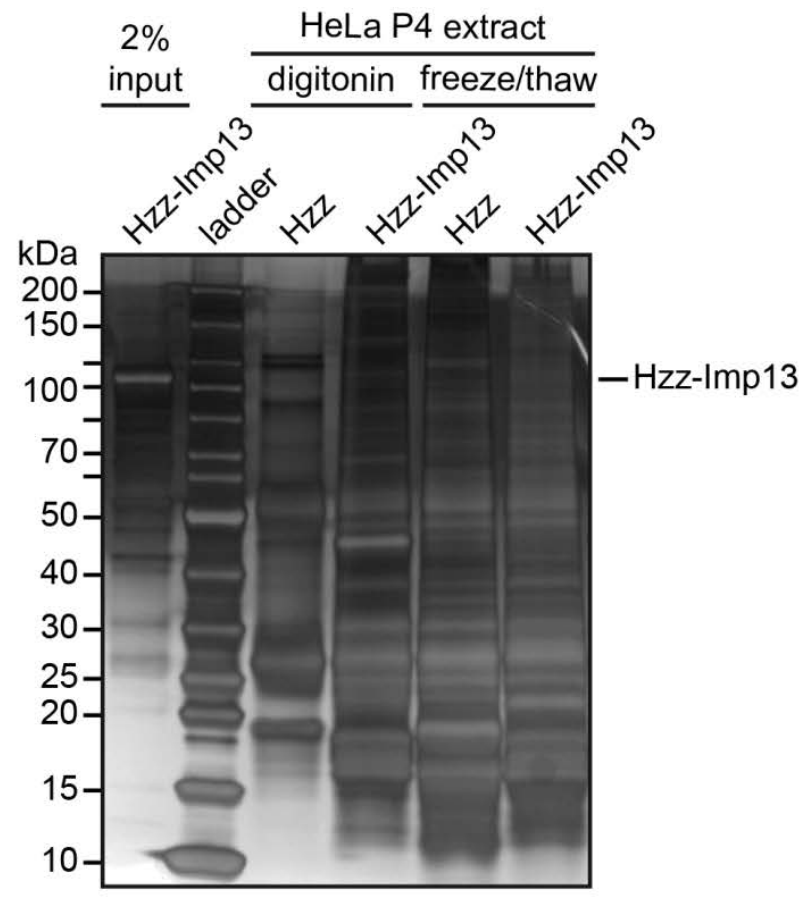

B

Hzz-tag

Hzz-tag

+ digitonin lysate

+ freeze/thaw lysate

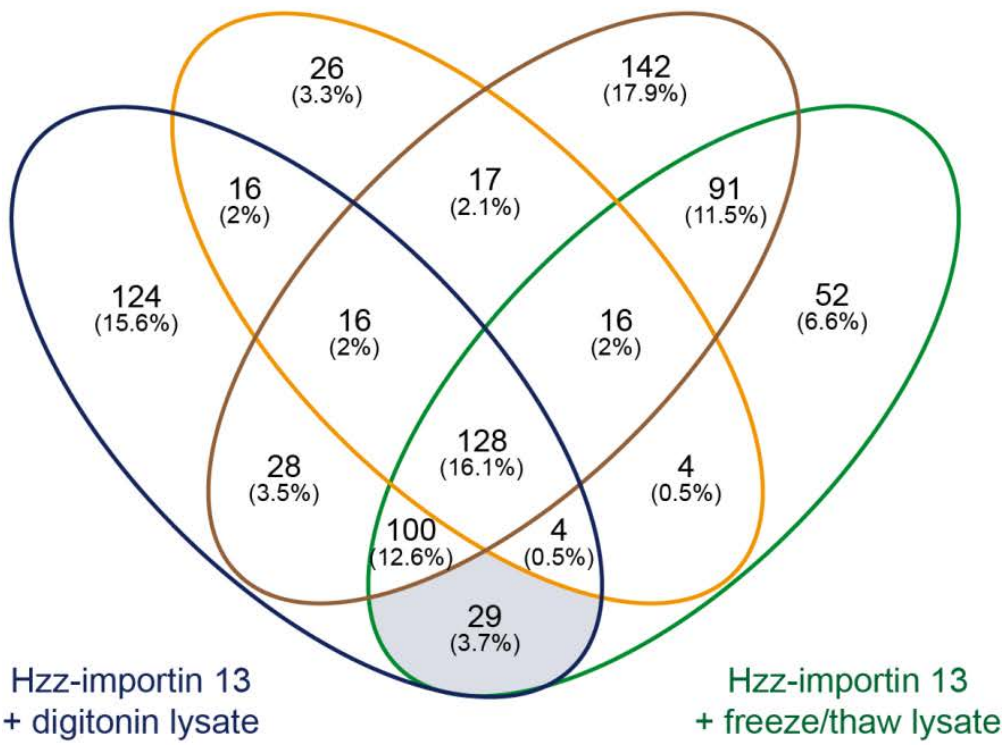

Figure 14: Proteins bound to Hzz-tag and Hzz-importin 13 from HeLa P4 cell extract. (A) Hzz-tag or Hzz-importin 13 was immobilized on IgG-Sepharose and incubated with digitonin or freeze/thaw HeLa P4 cell extract in transport buffer. Bound proteins were eluted with magnesium chloride and analyzed by SDS-PAGE, followed by silver staining. ladder: PageRuler Unstained Protein Ladder. (B) Venn diagram showing the overlap of proteins bound to Hzz-tag and Hzz-importin 13 from both digitonin and freeze/thaw HeLa P4 cell extract. See Table 10 for proteins that bound specifically to Hzz-importin 13 from both cell extracts (area shaded in light blue). 
Using a threshold of minimum 10 peptide spectrum matches (PSMs, total number of identified peptide spectra matched to a protein), a total of 445 proteins were identified by mass spectrometry that bound to Hzz-importin 13 from the digitonin HeLa P4 cell extract and 424 proteins that were bound from the freeze/thaw cell extract. Of these, only 29 proteins bound specifically to Hzz-importin 13 but not the Hzz-tag from both the digitonin and the freeze/thaw HeLa P4 cell lysate (Figure 14B, Table 10). Another 124 proteins were identified that specifically bound to importin 13 from the digitonin cell extract (Table S2) and 52 proteins that specifically bound to importin 13 from the freeze/thaw cell extract (Table S3).

Table 10: List of proteins that bound to Hzz-importin 13 from both a digitonin and a freezelthaw HeLa P4 cell extract (see Table S2 and Table S3 for cell extract specific proteins)

\begin{tabular}{|c|c|c|c|}
\hline Uniprot ID & Protein & Gene & Reference \\
\hline 094829 & Importin-13 & IPO13 & \\
\hline Q92538 & $\begin{array}{l}\text { Golgi-specific brefeldin A-resistance guanine nucleotide exchange } \\
\text { factor } 1\end{array}$ & GBF1 & \\
\hline P63279 & SUMO-conjugating enzyme UBC9 & UBE2I & (135) \\
\hline Q7Z3U7 & Protein MON2 homolog & MON2 & \\
\hline Q9NRF9 & DNA polymerase epsilon subunit 3 & POLE3 & (147) \\
\hline E9PS17 & $\mathrm{N}$-terminal kinase-like protein & SCYL1 & \\
\hline P61326 & Protein mago nashi homolog & MAGOH & (135) \\
\hline Q9Y5S9 & RNA-binding protein $8 \mathrm{~A}$ & RBM8A & (135) \\
\hline Q9H9A6 & Leucine-rich repeat-containing protein 40 & LRRC40 & \\
\hline 075420 & PERQ amino acid-rich with GYF domain-containing protein 1 & GIGYF1 & \\
\hline Q9NRG0 & Chromatin accessibility complex protein 1 & CHRAC1 & (147) \\
\hline Q9Y4H2 & Insulin receptor substrate 2 & IRS2 & \\
\hline Q8WUF5 & RelA-associated inhibitor & PPP1R13L & \\
\hline Q14160 & Protein scribble homolog & SCRIB & \\
\hline P27540 & Aryl hydrocarbon receptor nuclear translocator & ARNT & \\
\hline 043813 & LanC-like protein 1 & LANCL1 & \\
\hline E9PMS6 & LIM domain only protein 7 & LMO7 & \\
\hline J3KR24 & Isoleucine--tRNA ligase & IARS & \\
\hline Q7Z460 & CLIP-associating protein 1 & CLASP1 & \\
\hline P52655 & Transcription initiation factor IIA subunit 1 & GTF2A1 & \\
\hline F8W9S7 & GTPase-activating protein and VPS9 domain-containing protein 1 & GAPVD1 & \\
\hline Q16204 & Coiled-coil domain-containing protein 6 & CCDC6 & \\
\hline Q6P2H3 & Centrosomal protein of $85 \mathrm{kDa}$ & CEP85 & \\
\hline Q9BWH6 & RNA polymerase II-associated protein 1 & RPAP1 & \\
\hline B1ANR0 & Polyadenylate-binding protein & PABPC4 & \\
\hline Q9P1Y5 & Calmodulin-regulated spectrin-associated protein 3 & CAMSAP3 & \\
\hline F8W726 & Ubiquitin-associated protein 2-like & UBAP2L & \\
\hline P35249 & Replication factor $\mathrm{C}$ subunit 4 & RFC4 & \\
\hline P26373 & $60 S$ ribosomal protein L13 & RPL13 & \\
\hline
\end{tabular}


Of the 29 proteins that bound to importin 13 from both cell lysates, five proteins (Ubc9, POLE3, MAGOH, RBM8A, CHRAC1) were known importin 13 cargoes demonstrating that the established importin 13 binding experiment as well as both cell extraction methods allow for the specific identification of importin 13 substrates. Another three known importin 13 cargoes (eIF1A, POLE4, NFYC) were specifically identified for the digitonin cell lysate (Table S2) and four (NFYC, NFYB, DRAP1, DR1) for the freeze/thaw cell lysate (Table S3), showing that both cell extraction methods can complement each other. The export cargo elF1A was also identified in the mass spectrometry analysis to bind to Hzzimportin 13 from both the digitonin (24 PSMs) and the freeze/thaw cell lysate (5 PSMs), however, some unspecific binding to the Hzz-tag could also be detected.

Apart from the known importin 13 cargoes, several proteins were also found to interact with importin 13 that are involved in the regulation of the cytoskeleton (CLASP1, CCDC6, CAMSAP3) or the secretory pathway (GBF1, MON2). Nuclear transport receptors, such as Crm1, have previously been reported to actively sort cytoplasmic proteins from the nucleus (32, 157, 188-191).

The above experiment demonstrates that immobilization of importin 13 on beads and incubation with cell extract is an effective method to fish for importin 13 cargoes. In addition, the above findings suggest that a digitonin cell extract might be more effective in identifying importin 13 interaction partners than a freeze/thaw cell lysate, as with the digitonin cell lysate more proteins could be fished and less unspecific binding was detected. For this reason, the digitonin cell lysate was used in the SILAC based proteomics approach for the identification of importin 13 import and export cargoes (3.3.3). The newly identified proteins were not analyzed in further detail, as the SILAC experiments following in section 3.3.3 were expected to be more conclusive, allowing for the specific discrimination of importin 13 import and export cargoes. However, several of the proteins identified were also detected in the SILAC screen (Table S9), some of which were further characterized by overexpression experiments (3.3.3.4.3).

\subsubsection{Identification of Single Importin 13 Bound Proteins Affected by Ubc9 or Enriched from HeLa P4 Cell Extract}

A major limitation of the above mass spectrometry approach to identify proteins that bind to importin 13 from a HeLa P4 cell lysate (3.3.1) is that it does not allow for the distinction of importin 13 import and export cargoes. For this reason, binding experiments similar to the previous mass spectrometry experiment were established with the difference of 
including Ubc9 (known import cargo) or RanGTP $\mathrm{Q}_{\mathrm{Q} 9 \mathrm{~L}}$ in the binding reactions. Presence of exogenous RanGTP ${ }_{\mathrm{Q} 69 \mathrm{~L}}$ was expected to promote the binding of importin 13 export cargoes to importin 13 from a cell extract, while exogenous Ubc9 was expected to compete with both endogenous importin 13 import and export cargoes for binding to importin 13.

Importin 13 binding experiments were optimized using the known export cargo elF1A and the known import cargo Ubc9 as positive controls for binding of export and import cargoes, respectively. Various binding conditions were tested and optimized, including different binding buffers, protein concentrations, importin 13 fusion tags, cell extract preparation, cell extract amounts as well as different elution conditions. In these preliminary binding experiments, proteins could repeatedly be observed in Coomassie stained gels that were either significantly enriched from the cell lysate or whose binding to importin 13 was affected if Ubc9 or RanGTP $\mathrm{Q}_{6 \mathrm{~L}}$ were added to the binding reaction (an example is shown in Figure 15).

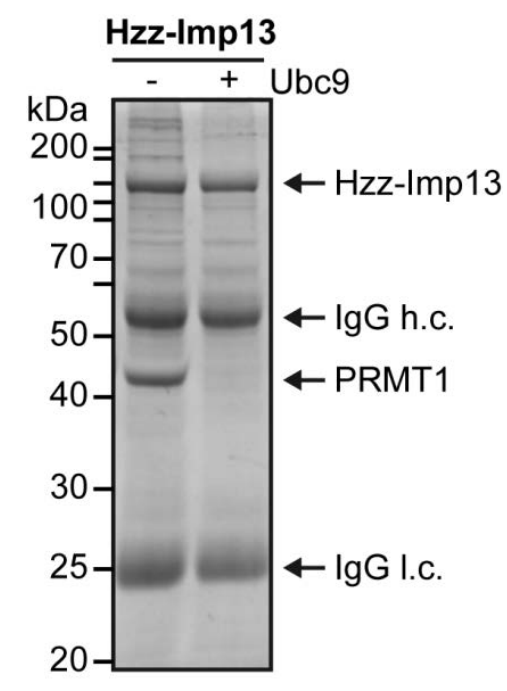

Figure 15: Example of importin 13 bound protein that is reduced in the presence of Ubc9. Hzz-importin $13(20 \mu \mathrm{g})$ was immobilized on IgG-Sepharose and incubated with a digitonin HeLa P4 cell extract in the absence or presence of $5 \mu \mathrm{g}$ Ubc9 in $20 \mathrm{mM}$ HEPES, pH7.5, $50 \mathrm{mM} \mathrm{NaCl}, 10 \%$ glycerol, $0.01 \%$ NP-40 buffer supplemented with $2 \mathrm{mg} / \mathrm{mL}$ BSA. Bound proteins were eluted by glycine elution and analyzed by SDS-PAGE, followed by Coomassie staining. PRMT1 was detected in the protein band that was reduced in the presence of Ubc9. I.c.: light chain; h.c.: heavy chain. 
Protein bands with altered protein amounts were excised, prepared for mass spectrometry and handed to our collaborators for mass spectrometry analysis. Raw data was analyzed with the Proteome Discoverer against a human protein database. However, in all of the analyzed protein bands, multiple proteins were identified by mass spectrometry, complicating the identification of a key protein. Nonetheless, seven proteins were selected (Table 11) that were in accordance with the expected molecular weight and analyzed in more detail.

Table 11: Importin 13 bound proteins enriched from a HeLa P4 cell extract or affected by exogenous Ubc9 addition ${ }^{\#}$

\begin{tabular}{|c|c|c|c|c|c|c|}
\hline $\begin{array}{l}\text { Uniprot } \\
\text { ID }\end{array}$ & Protein Name & Gene & $\begin{array}{c}\text { MW } \\
{[\mathrm{kDa}]}\end{array}$ & Comment $^{a}$ & $\begin{array}{l}\text { Imp13 co- } \\
\text { expression }^{\text {b }}\end{array}$ & SILAC $^{c}$ \\
\hline Q9BRQ8 & Apoptosis-inducing factor 2 & AIFM2 & 40.5 & $\begin{array}{l}\text { reduced by } \\
\text { Ubc9 }\end{array}$ & - & - \\
\hline P62140 & $\begin{array}{l}\text { Serine/threonine-protein phosphatase } \\
\text { PP1-beta catalytic subunit }\end{array}$ & PPP1CB & 37.2 & $\begin{array}{l}\text { reduced by } \\
\text { Ubc9 }\end{array}$ & ++ (export) & $\begin{array}{l}\text { import }^{d} \\
\text { export }^{d}\end{array}$ \\
\hline 095218 & $\begin{array}{l}\text { Zinc finger Ran-binding domain-containing } \\
\text { protein } 2\end{array}$ & ZRAB2 & 37.4 & $\begin{array}{l}\text { reduced by } \\
\text { Ubc9 }\end{array}$ & - & - \\
\hline E9PKG1 & Protein arginine $\mathrm{N}$-methyltransferase 1 & PRMT1 & 37.7 & $\begin{array}{l}\text { reduced by } \\
\text { Ubc9 }\end{array}$ & ++ (export) & export $^{\mathrm{e}, *}$ \\
\hline Q92879 & CUGBP Elav-like family member 1 & CELF1 & 52.0 & $\begin{array}{l}\text { enriched } \\
\text { from lysate }\end{array}$ & ++ (export) & - \\
\hline P60842 & Eukaryotic initiation factor $4 \mathrm{~A}-\mathrm{I}$ & EIF4A1 & 46.1 & $\begin{array}{l}\text { enriched } \\
\text { from lysate }\end{array}$ & + (import) & import $^{d}$ \\
\hline Q9Y265 & RuvB-like 1 & RUVBL1 & 50.2 & $\begin{array}{l}\text { enriched } \\
\text { from lysate }\end{array}$ & + (import) & import $^{\mathrm{e}}$ \\
\hline
\end{tabular}

\#: single proteins detected in different binding experiments that were enriched upon HeLa P4 cell lysate addition or affected by Ubc9 addition were analyzed by mass spectrometry. Proteins of the expected size were selected for further analysis. Proteins highlighted in bold were also detected in the SILAC screen (3.3.3), where Ubc9 reduced binding of PPP1CB, PRMT1, RUVBL1 and RanGTP ${ }_{\text {Q69 }}$ reduced binding of EIF4A1.

*: PRMT1 was detected in only one of the SILAC biological replicates.

a: single proteins whose binding to importin 13 was strongly enriched from cell lysate or whose binding was reduced in the presence of Ubc9; b: importin 13 effect in FLAG-importin 13 overexpression experiment; c: proteins that later also came up in the SILAC screen as import or export candidates; d: low confidence substrate; e: very low confidence substrate; -: no importin 13 effect; +: weak importin 13 effect; ++: importin 13 effect

For further analysis, proteins were cloned into different vector backbones and expressed as HA- and GFP-GST-fusion constructs in HeLa P4 cells and analyzed for any changes in subcellular localization upon importin 13 coexpression (Figure 16). 


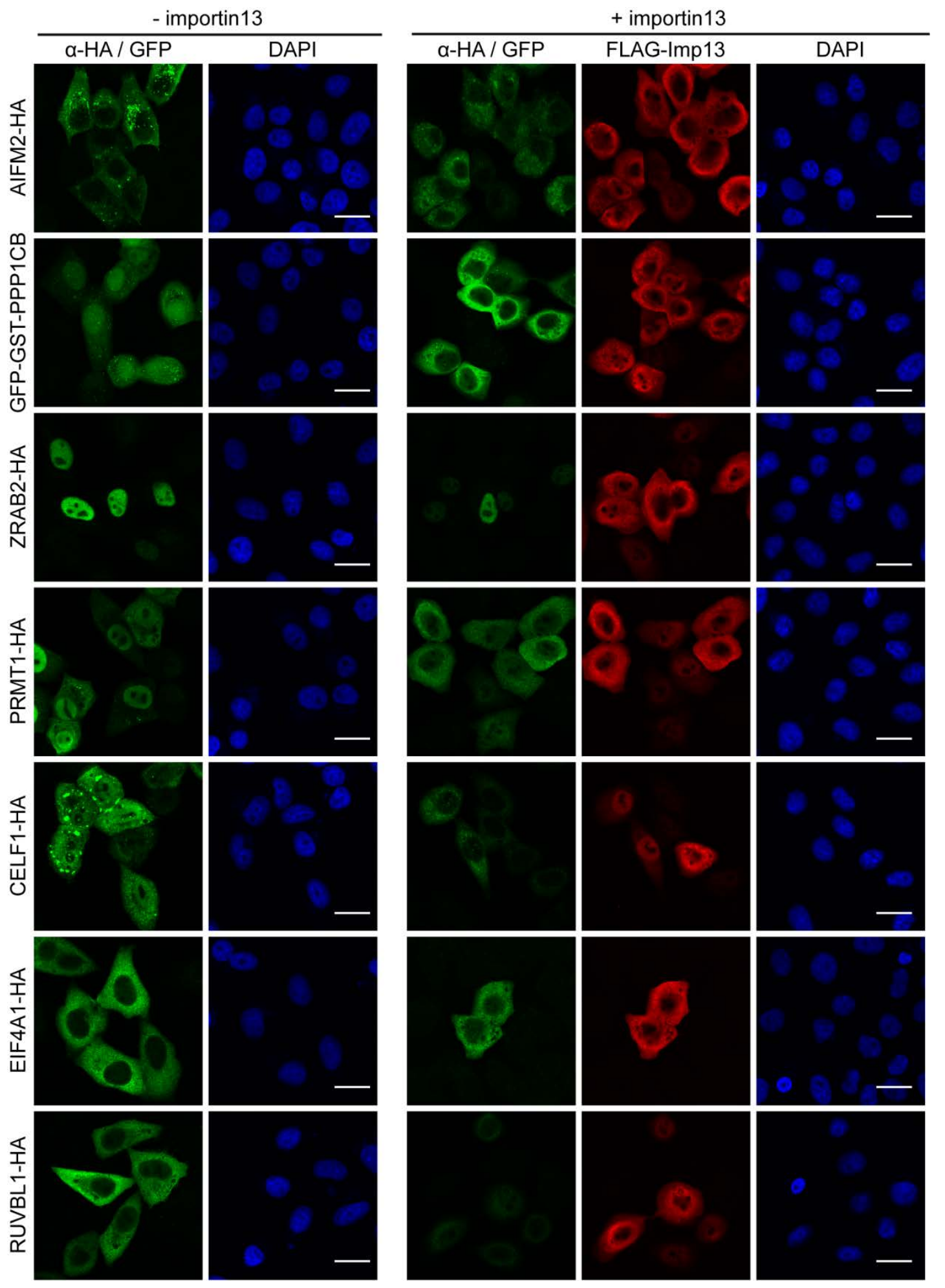

Figure 16: PPP1CB, PRMT1, CELF1, EIF4A1 and RUVBL1 change their subcellular localization upon importin 13 coexpression. HeLa P4 cells were transiently transfected with plasmids coding for HA- or GFP-GST-tagged proteins and optionally cotransfected with plasmids coding for FLAG-importin 13. Proteins were visualized by indirect immunofluorescence with an antiHA and anti-FLAG antibody. See Table 11 for protein details. The scale bars correspond to $20 \mu \mathrm{m}$. 
More than half of the proteins analyzed were affected by importin 13 coexpression. PRMT1 and CELF1 showed weak relocalization to the cytoplasm, while EIF4A1 and RUVBL1 showed weak relocalization to the nucleus, making them putative importin 13 export and import cargoes, respectively. Curiously, PPP1CA showed a relocalization to the nucleus if fused to an HA-tag and relocalization to the cytoplasm if fused to a GFP-GSTtag. However, with the exception of PPP1CB an importin 13 effect was only observed if the proteins were fused to a C-terminal HA-tag and not an N-terminal GFP-GST-tag (data not shown). The position and size of the GFP-GST-tag potentially prevent the proteins from interacting with importin 13. PPP1CB, EIF4A1 and RUVBL1 were later also identified as potential importin 13 import cargoes from a HeLa P4 cell lysate in the SILAC screen (3.3.3), albeit as low confidence cargoes.

In summary, PPP1CB, EIF4A1, RUVBL1, PRMT1 and CELF1 were affected by importin 13 overexpression, making them potential importin 13 cargoes. This demonstrates that pull-downs in the presence or absence of RanGTP ${ }_{\mathrm{Q} 69 \mathrm{~L}}$ or Ubc9 can allow for the specific identification of importin 13 interaction partners. To allow for comparative analysis of proteins bound to importin 13 from a HeLa P4 cell extract in the absence or presence of RanGTP ${ }_{\mathrm{Q} 69 \mathrm{~L}}$ or Ubc9, the binding experiments were coupled to a quantitative mass spectrometry approach using SILAC (3.3.3).

\subsubsection{Quantitative Mass Spectrometry Based Identification of Importin 13 Import and Export Cargoes Using SILAC}

As illustrated in section 3.3.1 and 3.3.2 mass spectrometry based importin 13 binding assays are effective methods for the identification of importin 13 interaction partners. However, analysis of HeLa P4 proteome binding to immobilized importin 13 alone does not yield a clear answer to the question of whether a bound protein is a potential importin 13 import or export cargo. As highlighted in section 3.3.2 addition of Ubc9 or RanGTP Q69L $_{\text {to }}$ the pull-down reaction can facilitate the specific identification of importin 13 import or export cargoes. To allow for the quantitative comparison of proteins bound to Hzzimportin 13 from a HeLaP4 cell extract in the absence of presence of Ubc9 or RanGTP ${ }_{\mathrm{Q} 69 \mathrm{~L}}$, triple stable isotope labeling with amino acids in cell culture (SILAC) experiments were performed. 


\subsubsection{Pull-down Based Identification of Potential Importin 13 Cargoes Using SILAC}

To get a comprehensive list of importin 13 interaction partners and to allow for the specific distinction of importin 13 import and export cargoes, pull-down assays were performed in

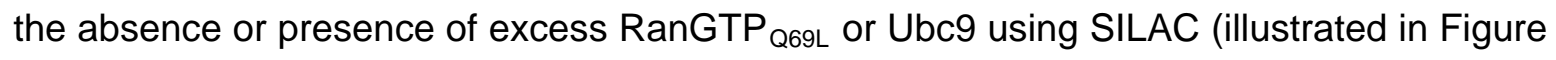
17).

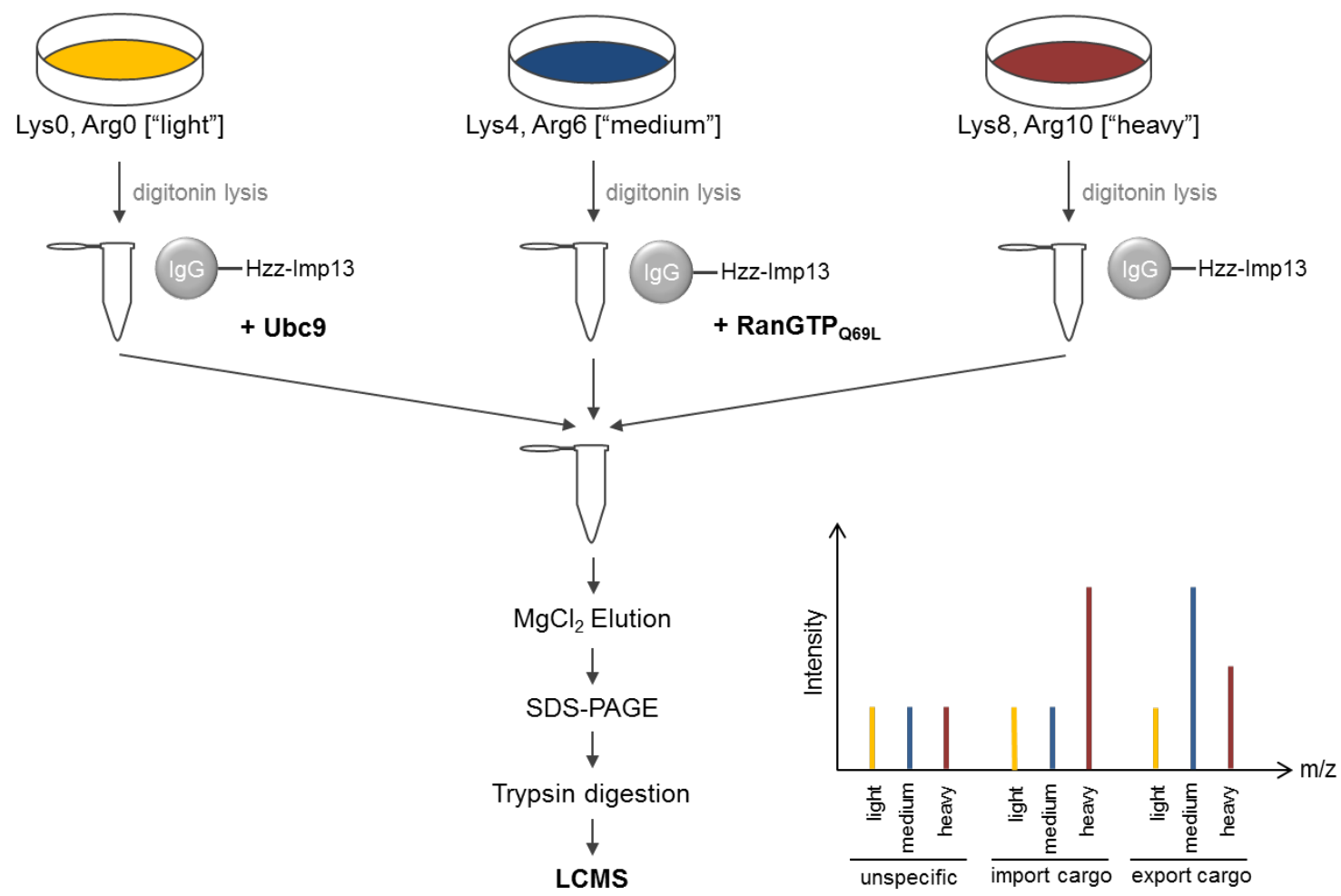

Figure 17: Experimental workflow of SILAC screen. Hzz-tagged importin 13 was immobilized on IgG-Sepharose and incubated with digitonin cell extracts of HeLa P4 cells grown in DMEM medium containing light (Lys0, Arg0), medium (Lys4, Arg6) or heavy (Lys8, Arg10) amino acids. One binding reaction was performed in the presence of cell extract alone, a second reaction was performed in the presence of RanGTP $\mathrm{Q}_{9 \mathrm{~L}}$ to favor the binding of importin 13 export cargoes and a third reaction was performed in the presence of Ubc9 to prevent the formation of importin 13 import and export complexes. After binding, all three reactions were pooled and bound proteins were eluted with magnesium chloride and separated by SDS-PAGE. Peptides were extracted by in-gel tryptic digestion, analyzed by liquid chromatography-mass spectrometry and raw data was processed using MaxQuant. The graph in the lower right corner depicts a schematic mass spectrometry output for the above exemplified labeling experiment with each peptide appearing as a triplet with distinct mass differences. 
For all three binding reactions, Hzz-importin 13 was immobilized on IgG-Sepharose and incubated with differently labeled HeLa P4 cell extracts (Figure 18A). HeLa P4 cells were isotopically labeled by cultivating them in light, medium or heavy SILAC media that contained unlabeled L-lysine and L-arginine (Lys0, Arg0), 4,4,5,5-D4-L-Lysine and ${ }^{13} \mathrm{C}_{6}$-L-arginine (Lys4, Arg6) or ${ }^{13} \mathrm{C}_{6}{ }^{15} \mathrm{~N}_{2}$-L-lysine and ${ }^{13} \mathrm{C}_{6}{ }^{15} \mathrm{~N}_{4}$-L-arginine (Lys8, Arg10), respectively. Cells were lysed with digitonin and precleared with phenyl-Sepharose to selectively deplete endogenous nuclear transport receptors (27), as in previous mass spectrometry experiments (section 3.3.1 and data not shown) other nuclear transport receptors could be detected that likely were pulled out with importin 13 bound nucleoporins. As shown in Figure 18C (see Figure S3C, Figure S4C for label-swap experiments), importin 13, importin $\beta$ and transportin were effectively removed from the cell extract by preincubation with phenyl-Sepharose. Depletion of these proteins had only minor effects on endogenous importin 13 cargo concentrations as tested for Ubc9 and elF1A. In addition, the cell lysates were precleared with Hzz-IgG-Sepharose, to remove proteins binding unspecifically to the affinity matrix.

One binding reaction was performed in the presence of the known importin 13 import cargo Ubc9, as Ubc9 binding to importin 13 is expected to prevent the formation of other importin 13 import and export complexes. The importin 13 binding site for Ubc9 overlaps with the binding site for RanGTP, thus Ubc9 added in excess should compete with RanGTP for binding to importin 13 and prevent the formation of importin 13 export complexes. In addition, excess Ubc9 is expected to prevent the formation of importin 13 import complexes by either competing with other import cargoes for the same binding site or by preventing their binding to non-overlapping binding sites due to steric clashes. A second reaction was performed in the presence of RanGTP ${ }_{Q 69 L}$, a Ran mutant that is impaired in RanGTP hydrolysis and is expected to facilitate the binding of export cargoes and trap them as stable complexes. In the third reaction, no exogenous Ubc9 or RanGTP $_{\mathrm{Q} 69 \mathrm{~L}}$ but only cell lysate was added, to enrich for importin 13 import complexes. Export complexes are not expected to be enriched, as only RanGDP and not RanGTP should be present in the cell lysate, due to the activity of cytoplasmic RanGAP. 


\section{A SILAC binding reactions:}

1. Hzz-importin 13 + light HeLa P4 cell extract (I) + Ubc9

2. Hzz-importin $13+$ medium HeLa P4 cell extract $(m)+$ RanGTP $_{\text {Q69L }}$

3. Hzz-importin 13 + heavy HeLa P4 cell extract (h)

B

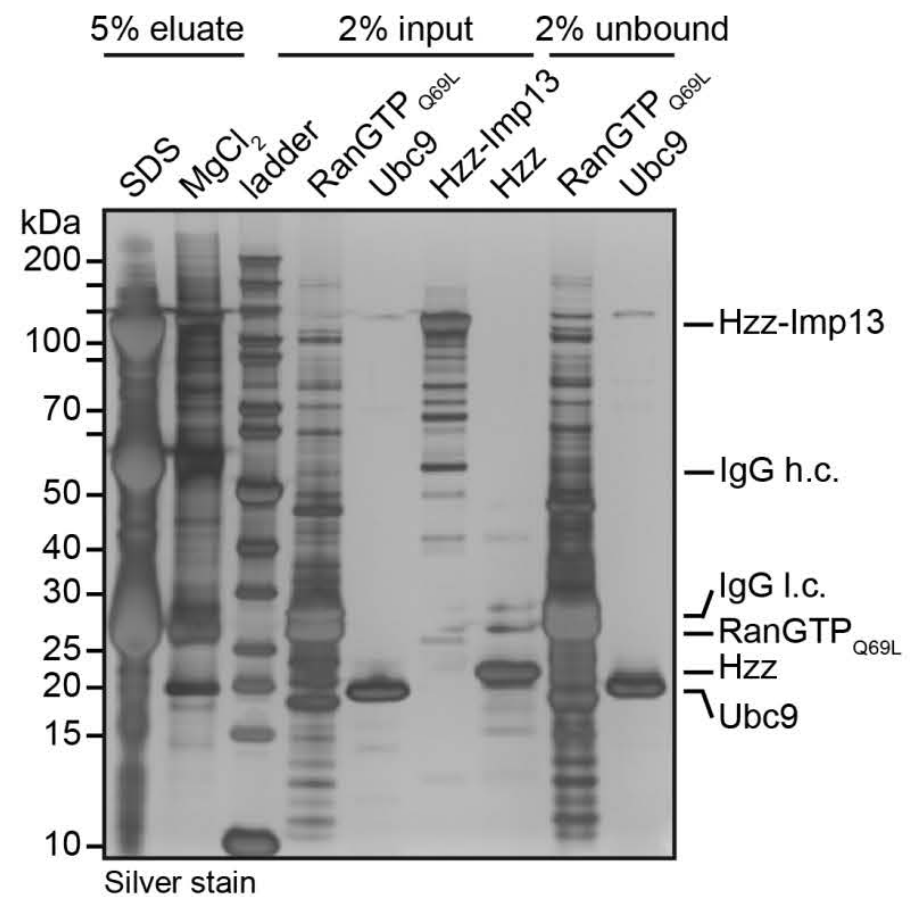

C

$5 \%$ eluate

$1 \%$ HeLa P4 lysate
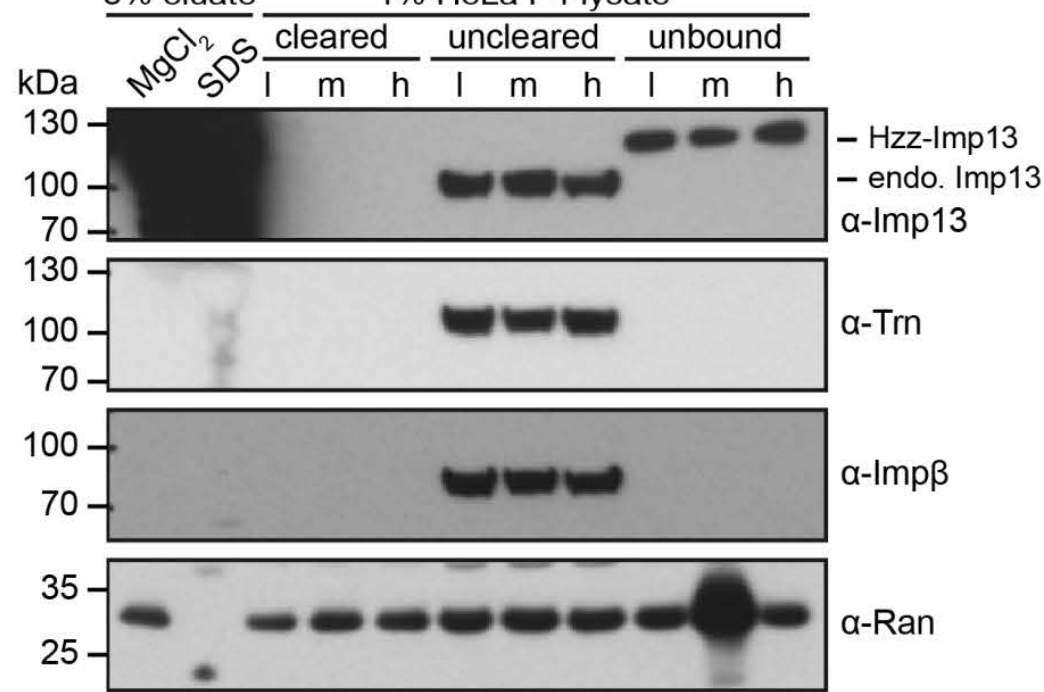

a-Ran

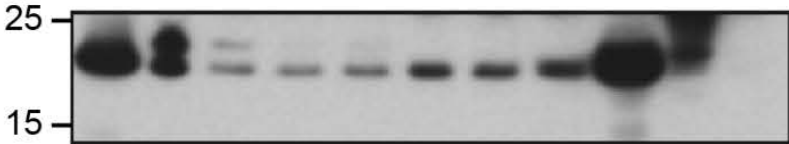

a-Ubc9

25

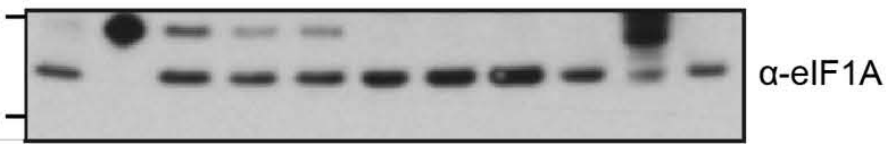


Figure 18: SILAC binding reactions and phenyl-Sepharose depletion of HeLa P4 cell extracts. (A) Schematic showing exemplary SILAC binding reactions. (B, C) Hzz-tagged importin 13 $(0.5 \mathrm{nmol})$ was immobilized on IgG-Sepharose and incubated with cell extracts of HeLa P4 cells grown in DMEM medium containing light, medium or heavy amino acids in the absence or presence of $10 \mu \mathrm{M}$ RanGTP Q69L $_{\text {or }} 5 \mu \mathrm{M}$ Ubc9 in transport buffer. Cell extracts (uncleared) were precleared with Hzz/lgG-Sepharose and phenyl-Sepharose (cleared) to reduce unspecific interactions. Bound proteins were eluted in a first elution step with magnesium chloride, followed by a second elution step with $4 x$ SDS sample buffer. Cell lysates and eluted proteins were separated by SDS-PAGE and analyzed by silver staining (B) or immunoblotting with an anti-importin 13, anti-transportin, antiimportin $\beta$, anti-Ran, anti-Ubc9 and anti-eIF1A antibody (C). Note that in the unbound samples free Hzz-importin 13 can be detected as well as exogenous RanGTP ${ }_{\mathrm{Q} 69 \mathrm{~L}}$ and Ubc9 that were added in excess to the binding reactions with medium and light isotopically labeled cell lysates, respectively. See Figure S3 and Figure S4 for replicate experiments. ladder: PageRuler Unstained Protein Ladder; $\mathrm{MgCl}_{2}$ : magnesium chloride eluate; SDS: 4x SDS sample buffer eluate; I: light; m: medium; h: heavy; endog.: endogenous.

To allow for quantitative comparison of the three binding reactions, a triple SILAC experiment was performed using light, medium and heavy isotopically labeled digitonin cell extracts. As the differential labeling of the cells allows for later distinction of each binding reaction by mass spectrometry, the IgG-Sepharose beads from all three binding reactions were pooled for protein elution to ensure homogenous elution of bound proteins with magnesium chloride. Rather mild elution conditions were essential to avoid extensive co-elution of the IgG light and heavy chain of the IgG-Sepharose. Eluted proteins were analyzed by SDS-PAGE, followed by silver staining, which confirmed that multiple proteins had bound to Hzz-importin 13 and that they were sufficiently eluted by magnesium chloride elution (Figure 18B, see Figure S3B and Figure S4B for replicates). Further, Ubc9 and RanGTP $_{\mathrm{Q} 69 \mathrm{~L}}$ were significantly enriched in the eluate, showing that the recombinant proteins Ubc9 and RanGTP ${ }_{\mathrm{Q} 69 \mathrm{~L}}$ were functional and had bound efficiently to importin 13. For quantitative mass spectrometry, eluted proteins were separated by SDS-PAGE, followed by peptide extraction using in-gel tryptic digestion and analysis by liquid chromatography-mass spectrometry (LC-MS). LC-MS was performed by the Service Unit LCMS Protein Analytics (Dr. Oliver Valerius and Dr. Kerstin Schmitt), Georg-AugustUniversität Göttingen. Raw LC-MS data was searched against a human protein database using the MaxQuant software and the MaxQuant output data was analyzed with the Perseus software. For a detailed workflow of the Perseus analysis of importin 13 export and import cargoes see supplemental Table S4 and Table S5, respectively. 


\subsubsection{SILAC Based Importin 13 Pull-downs Selectively Identify Importin 13 Cargoes}

Three biological replicates of the SILAC based binding assays were performed including label-swap experiments to increase the specificity of the identified proteins and to exclude experimental bias. In total, 1, 224 proteins were identified by mass spectrometry to bind to Hzz-importin 13 in the triple SILAC experiment. As SILAC is a quantitative approach, differences in protein amounts bound to Hzz-importin 13 in the absence or presence of RanGTP $_{\mathrm{Q} 69 \mathrm{~L}}$ and Ubc9 can be detected by mass spectrometry. To assess how many proteins are enriched in their binding to importin 13 compared to binding reactions with importin 13 and excess RanGTP ${ }_{\mathrm{Q} 69 \mathrm{~L}}$ (Imp13/Imp13+Ran) or Ubc9 (Imp13/Imp13+Ubc9), normalized log2 SILAC ratios were calculated for each protein and visualized using scatter plots (Figure 19). The majority of the quantified proteins were distributed around logarithmic fold change values close to zero showing that the bulk part of the identified proteins were not affected by RanGTP ${ }_{\mathrm{Q} 69 \mathrm{~L}}$ or Ubc9 in their binding to importin 13. However, several proteins could be detected that were enriched several fold for the Imp13/Imp13+Ran ratio and even more for the Imp13/Imp13+Ubc9 ratio, identifying them as possible importin 13 substrates. Furthermore, enrichment was observed for binding reactions in the presence of $\operatorname{RanGTP}_{\mathrm{Q} 69 \mathrm{~L}}$, which is expected to facilitate the formation of importin 13 export complexes. Enrichment could also be observed in the presence of Ubc9, however, these proteins are likely unspecific binding proteins or possibly correspond to Ubc9 interaction partners. 


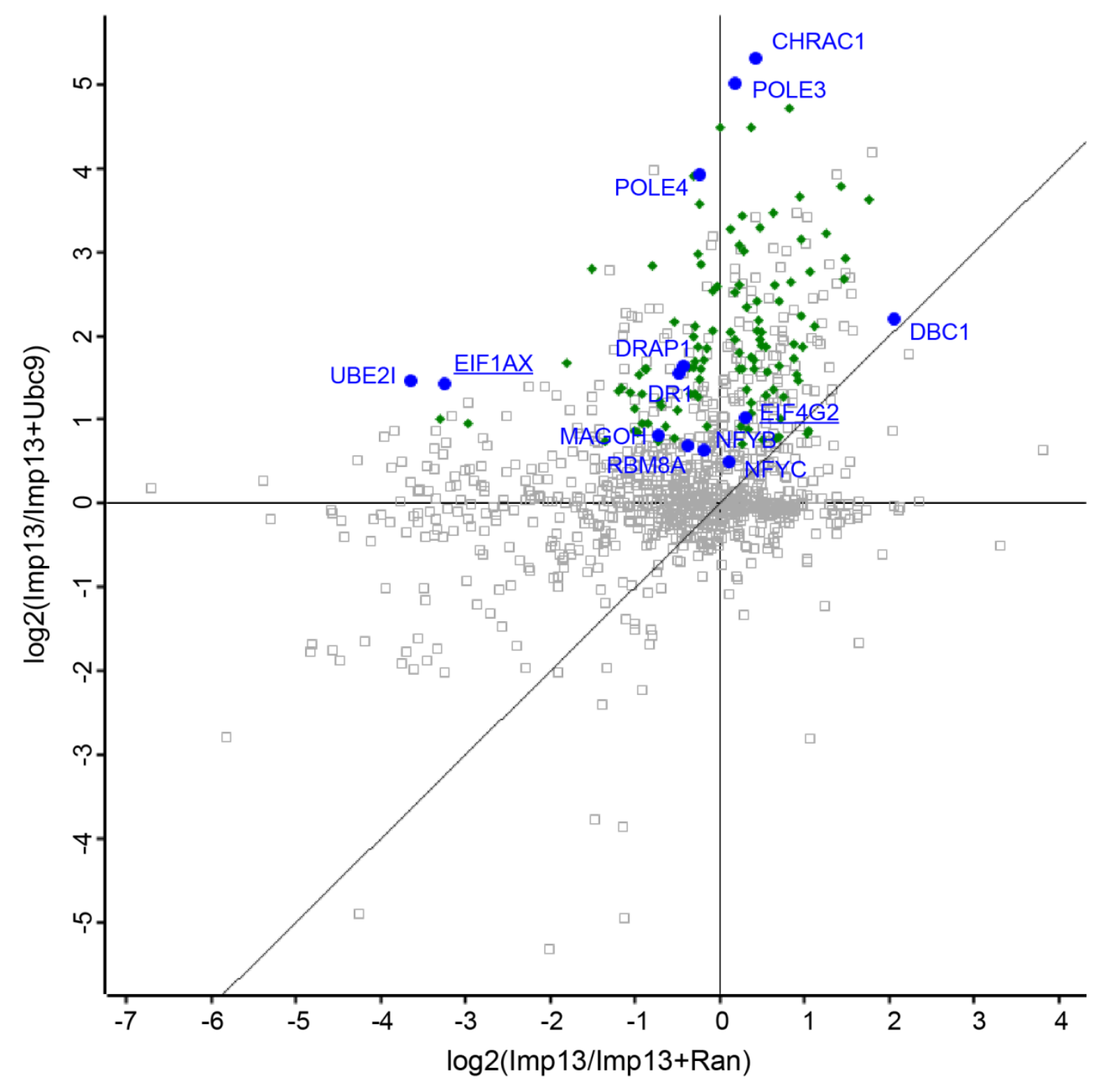

Figure 19: Putative and known importin 13 cargoes identified in SILAC screen (Imp13/Imp13+Ran vs. Imp13/Imp13+Ubc9). All proteins detected (grey squares) in the triple SILAC experiment by mass spectrometry (3.3.3.1) were plotted as log2(Imp13/Imp13+Ubc9) against log2(Imp13/Imp13+Ran) ratios. Ratios were averaged from three SILAC biological replicate experiments. Blue circles: known importin 13 substrates (underlined proteins are known export cargoes, the others are known import cargoes). Green diamonds: proteins detected by mass spectrometry with a $\log 2(\mathrm{Imp} 13 / \mathrm{Imp} 13+U \mathrm{bc} 9) \geq 0.5$ for all three biological replicates. Proteins with an average $\log 2(\operatorname{Imp} 13 / \mathrm{Imp} 13+\mathrm{Ubc} 9) \geq 0.5$ that are not depicted in green were quantified with a lower SILAC ratio in at least one of the replicates. Note that the distribution of Ubc9 (UBE2I) is not representative as exogenous unlabeled Ubc9 was present in one of the binding reactions with unlabeled cell lysate and therefore was also quantified by mass spectrometry. 
As a first validation of the SILAC based assay, proteins quantified by mass spectrometry were queried for known importin 13 cargoes (Table 1). More than half of the reported cargoes were detected in the SILAC screen (Table 12, Figure 19), proving the utility of the assay for the identification of novel importin 13 substrates. Known importin 13 import cargoes showed a significant enrichment for the Imp13/Imp13+Ubc9 ratio with average log2 SILAC ratios of 0.5 to 5.3 (Table 12, Figure 19). However, with the exception of DBC-1 (KIAA1967), which is only established as an importin 13 binding partner but not as a cargo, no or only minor enrichments for the Imp13/Imp13+Ran ratio could be detected, indicating that the Imp13/Imp13+Ubc9 ratio is more useful for the identification of novel import cargoes than the Imp13/Imp13+Ran ratio. Interestingly, the known heterodimeric importin 13 import cargoes MAGOH/RBM8A, CHRAC1/POLE3, POLE3/POLE4, DRAP1/DR1 and NFYB/NFYC $(135,141,146,147)$ showed a similar enrichment for both subunits of the heterodimer, confirming previous findings that these proteins are imported by importin 13 as heterodimers. While the NFYB/NFYC heterodimer was enriched 1.4-fold with a $\log 2(\operatorname{Imp} 13 / \mathrm{Imp13}+\mathrm{Ubc9})$ ratio of $\sim 0.5$, the CHRAC1/POLE3 heterodimer had a $\log 2(\operatorname{Imp} 13 / \mathrm{Imp} 13+\mathrm{Ubc9}$ ) ratio of $\sim 5.2$, corresponding to a more than 35 -fold enrichment. The subunits of the CHRAC1/POLE3 heterodimer were also the only proteins that showed enrichment for the Imp13/Imp13+Ran ratio with a log2 SILAC ratio of $\sim 1.0$. Note that the $\log 2$ SILAC ratios for Ubc9 are highly variable but this was to be expected as exogenous unlabeled Ubc9 was present in one of the reactions. In the first SILAC replicate (SILAC1), the unlabeled Ubc9 was added to the light HeLa P4 cell extract and therefore in this sample not only endogenous Ubc9 but also recombinant Ubc9 was measured, resulting in a negative log2(Imp13/Imp13+Ubc9) ratio. Apart from Ubc9 all other proteins showed similar log2 SILAC ratios for all three biological replicates, showing that there is only a low variability between the individual SILAC experiments. 
Table 12: Known importin 13 cargoes that were identified in the SILAC screen

\begin{tabular}{|c|c|c|c|c|c|c|c|c|c|c|c|}
\hline \multicolumn{12}{|c|}{ Export cargoes } \\
\hline \multirow[b]{2}{*}{ Uniprot_ID } & \multirow[b]{2}{*}{ Imp13 interactor } & \multirow[b]{2}{*}{ MW [kDa] } & \multicolumn{5}{|c|}{$\log 2(\operatorname{Im} p 13+\operatorname{Ran} / \operatorname{Im} \mathrm{p} 13)$} & \multicolumn{4}{|c|}{$\log 2(\operatorname{Im} p 13+\operatorname{Ran} / \operatorname{lm} \mathrm{p} 13+\mathrm{Ubc} 9)$} \\
\hline & & & SILAC1 & SILAC2 & SILAC3 & Average & $-\log (p \text { value })^{\star}$ & SILAC1 & SILAC2 & SILAC3 & $-\log (p \text { value })^{\star}$ \\
\hline P47813 & elF1A (EF1A) & 16.5 & 3.26687 & 3.30372 & 3.18652 & 3.25237 & 4.24736 & 5.01948 & 4.67779 & 4.82963 & 4.8423 \\
\hline P78344 & EIF4G2 & 102.4 & -0.422233 & -0.416948 & -0.0333701 & -0.29085 & 0.03442 & -0.242226 & 0.513976 & 0.941697 & 0.404482 \\
\hline
\end{tabular}

\begin{tabular}{|c|c|c|c|c|c|c|c|c|c|c|c|c|}
\hline \multicolumn{13}{|c|}{ Import cargoes } \\
\hline \multirow[b]{2}{*}{ Uniprot_ID } & \multirow[b]{2}{*}{ Imp13 interactor } & \multirow[b]{2}{*}{ MW [kDa] } & \multicolumn{5}{|c|}{$\log 2(\operatorname{Im} \mathrm{p} 13 / / \mathrm{m} \mathrm{p} 13+\operatorname{Ran})$} & \multicolumn{5}{|c|}{$\log 2(\operatorname{Im~p13/Im~p13+Ubc9)~}$} \\
\hline & & & SILAC1 & SILAC2 & SILAC3 & Average & $-\log (p \text { value })^{\star}$ & SILAC1 & SILAC2 & SILAC3 & Average & $-\log (p \text { value })^{\star}$ \\
\hline P63279 & UBE2I (Ubc9) & 18.0 & 0.0117819 & -4.91737 & -6.06226 & -3.65595 & 0.0430585 & -4.26446 & 3.72448 & 4.94542 & 1.46848 & 0.480393 \\
\hline P61326 & MAGOH (Mago) & 17.2 & -1.08381 & -0.378907 & -0.711086 & -0.724602 & 0.0156264 & 0.811224 & 0.665752 & 0.965643 & 0.814207 & 2.25492 \\
\hline F419J7 & RBM8A (Y14) & 19.9 & -0.249994 & -0.241612 & -0.675765 & -0.389124 & 0.0252879 & 0.773152 & 0.480539 & 0.831101 & 0.694931 & 1.92964 \\
\hline Q9NRG0 & CHRAC1 (CHRAC15) & 14.7 & 0.489029 & 0.683113 & 0.0801119 & 0.417418 & 1.14478 & 5.17273 & 5.3355 & 5.43066 & 5.31296 & 3.99816 \\
\hline Q9NRF9 & POLE3 (CHRAC17) & 16.9 & 0.207143 & 0.297602 & 0.00201835 & 0.168921 & 1.01523 & 4.47521 & 5.41648 & 5.16576 & 5.01915 & 2.80557 \\
\hline Q9NR33 & POLE4 (p12) & 12.2 & -0.156965 & -0.191327 & -0.380935 & -0.243075 & 0.0161895 & 3.4194 & 3.88773 & 4.45267 & 3.91994 & 2.54084 \\
\hline P25208 & NFYB (NF-YB) & 22.8 & 0.00733906 & 0.209391 & -0.762314 & -0.181861 & 0.155389 & 0.62938 & 0.385205 & 0.91937 & 0.644652 & 1.57798 \\
\hline Q13952 & NFYC (NF-YC) & 50.3 & -0.185179 & 0.126841 & 0.385707 & 0.109123 & 0.540255 & 0.46069 & 0.285284 & 0.732332 & 0.492769 & 1.50084 \\
\hline Q14919 & DRAP1 (NC2 $\alpha)$ & 22.4 & -0.0442855 & -0.266264 & -0.993838 & -0.434796 & 0.062666 & 1.27023 & 1.68302 & 1.99166 & 1.6483 & 2.10521 \\
\hline Q01658 & DR1 (NC2 $\beta)$ & 19.4 & -0.118335 & -0.285108 & -1.03673 & -0.480059 & 0.053382 & 1.21114 & 1.55211 & 1.87483 & 1.54603 & 2.12448 \\
\hline Q8N163 & KIAA1967 (DBC-1) & 102.9 & 2.05196 & $\mathrm{NaN}$ & $\mathrm{NaN}$ & 2.05196 & 0 & 2.20655 & $\mathrm{NaN}$ & $\mathrm{NaN}$ & 2.20655 & 0 \\
\hline
\end{tabular}

*right sided, one sample t-test $(p \leq 0.01)$, which tested the hypothesis that the log2 SILAC ratios w ere not equal to the value zero 
As export cargoes are expected to be enriched in binding reactions with RanGTP $\mathrm{Q}_{\mathrm{Q} 9 \mathrm{~L}}$

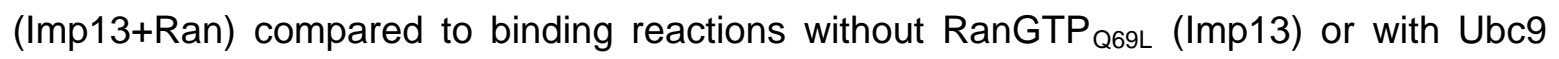
(Imp13+Ubc9), the $\log 2(\operatorname{Imp} 13+\mathrm{Ran} / \mathrm{Imp13})$ ratio was plotted against the $\log 2(\operatorname{Imp} 13+$ Ran/Imp13+Ubc9) ratio to also allow for an assessment of the Imp13+Ran/Imp13+Ubc9 ratio (Table 12, Figure 20). The first importin 13 export cargo to be reported was the translation initiation factor eIF1A, suggesting that it is one of the major importin 13 substrates and indeed elF1A was identified as one of the top hits. It was enriched by more than 20-fold with an average log2 SILAC ratio for Imp13+Ran/Imp13 and Imp13+Ran/Imp13+Ubc9 of 4.2 and 4.8, respectively. Of the two other reported importin 13 export cargoes, EIF4G2 but not HMG20A was identified (Table 12). EIF4G2 was detected with a log2 SILAC ratio between 0.5 to 1.0 for the Imp13+Ran/Imp13+Ubc9 ratio for two of the replicates and a negative value for the third replicate, whereas for the Imp13+Ran/Imp13 ratio no enrichment could be detected. Similar to elF1A, multiple of the newly identified proteins were enriched for both the Imp13+Ran/Imp13 and the Imp13+Ran/Imp13+Ubc9 ratio, making them promising importin 13 export candidates (Figure 20). Apart from the known export cargoes elF1A and EIF4G2 also RanBP1 was identified as a high scoring export cargo. RanBP1 can directly bind to RanGTP and together with RanGAP initiates the disassembly of export complexes (58, 59). Consequently, RanBP1 was either indirectly identified through its binding to RanGTP or possibly it is an importin 13 export cargo. In this regard, it has been shown that RanBP1 can be exported by Crm1 $(82,191)$. This finding suggests that possibly apart from importin 13 export cargoes also RanGTP binding proteins might be identified in the SILAC screen. 


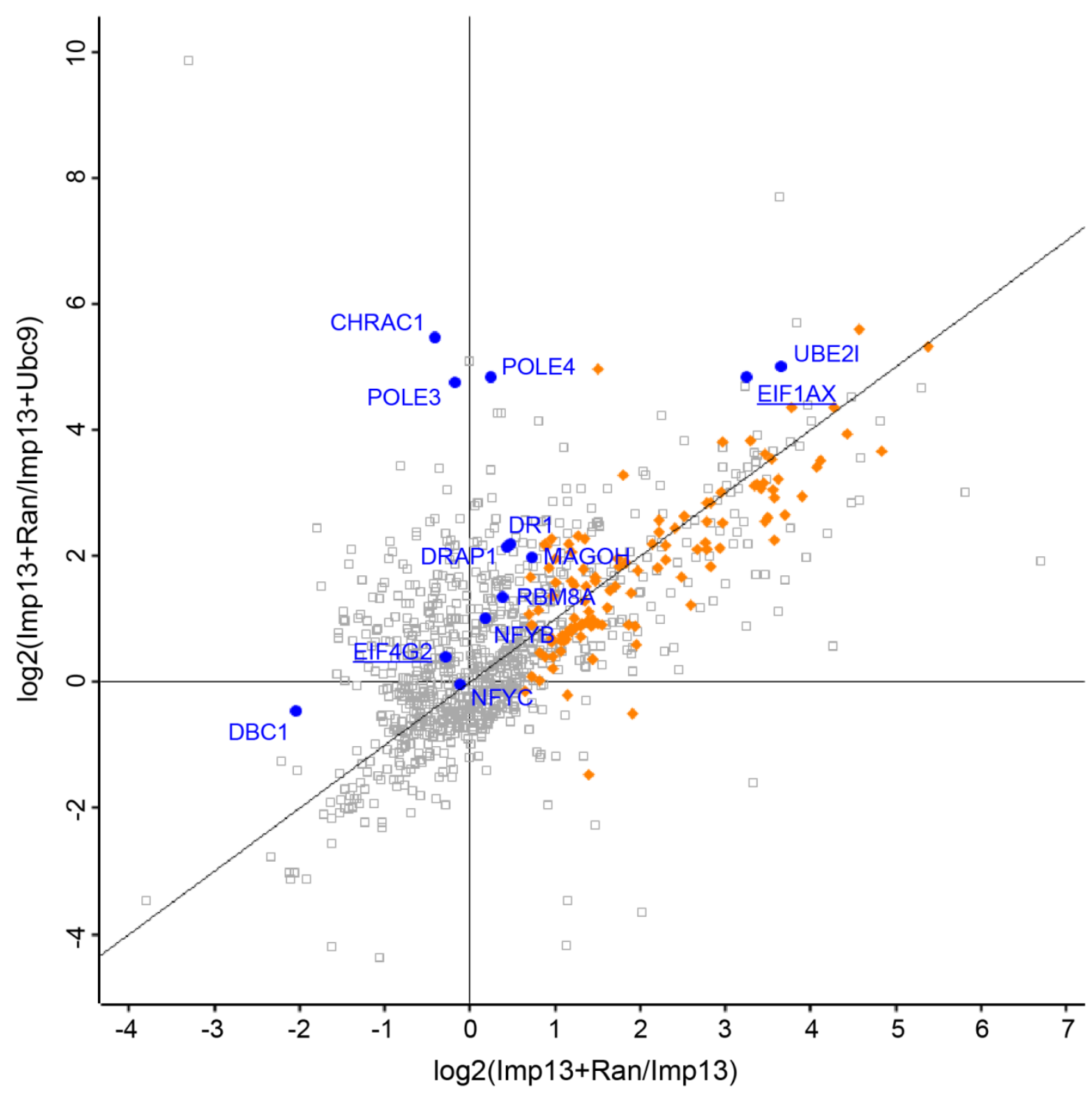

Figure 20: Putative and known importin 13 cargoes identified in SILAC screen (Imp13+Ran/Imp13 vs. Imp13+Ran/Imp13+Ubc9). All proteins detected (grey squares) in the triple SILAC experiment by mass spectrometry were plotted as log2(Imp13+Ran/Imp13+Ubc9) against $\log 2(\operatorname{Imp} 13+\mathrm{Ran} / \mathrm{Imp13})$ ratios. Ratios were averaged from three SILAC biological replicate experiments. Blue circles: known importin 13 substrates (underlined proteins are known export cargoes, the others are known import cargoes). Orange diamonds: proteins detected by mass spectrometry with a $\log 2(\operatorname{Imp} 13+R a n / I m p 13) \geq 0.5$ for all three biological replicates. Proteins with an average $\log 2(\operatorname{Imp} 13+\mathrm{Ran} / \mathrm{Imp} 13) \geq 0.5$ that are not depicted in orange were quantified with a lower SILAC ratio in at least one of the replicates. Note that the distribution of Ubc9 (UBE2I) is not representative as exogenous unlabeled Ubc9 was present in one of the binding reactions with unlabeled cell lysate and therefore was also quantified by mass spectrometry. 
The above findings demonstrate that the SILAC based assay is an effective method to screen for novel importin 13 substrates, as several known importin 13 cargoes were

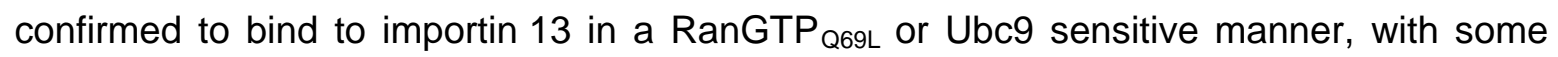
even showing a more than 20-fold enrichment. While export cargoes appear to be effectively enriched for both the Imp13+Ran/Imp13 and the Imp13+Ran/Imp13+Ubc9 ratio, known import cargoes were only enriched for the Imp13/Imp13+Ubc9 but not the Imp13/Imp13+Ran ratio. Based on the findings for the known importin 13 cargoes, filtering criteria were generated to group proteins quantified in the SILAC screen into potential importin 13 import and export cargoes (see section 3.3.3.3 for details).

\subsubsection{Filtering Criteria for the Identification of Importin 13 Import and Export Cargo Candidates}

Several filtering criteria were applied on the SILAC data, to allow for the specific identification of importin 13 cargoes. Proteins were only considered to be significantly enriched if they had a normalized log2 SILAC ratio $\geq 0.5$ in all three SILAC replicate experiments, which corresponds to an enrichment of minimum 1.4-fold. These filtering criteria were selected based on the previous observations that all known importin 13 cargoes showed a similar enrichment between all three biological replicates with log2 SILAC ratios of minimum 0.5. Additionally, a right-sided, one sample $t$-test with a threshold p-value of $\leq 0.01$ was applied, which tested the hypothesis that the log2 SILAC ratios were not equal to the value zero.

All identified known importin 13 cargoes showed enrichment for binding reactions with importin 13 alone compared to binding reaction with importin 13 and excess Ubc9 (Imp13/Imp13+Ubc9) but not compared to binding reactions with excess RanGTP ${ }_{\mathrm{Q} 69 \mathrm{~L}}$ (Imp13/Imp13+Ran). Thus, quantified proteins were considered to be import cargoes if they showed enrichment for the Imp13/Imp13+Ubc9 ratio. For further validation, proteins were also selected that showed enrichment for Imp13/Imp13+Ran to ensure that potential importin 13 cargoes were not omitted. Using these filtering criteria, a total of 17 proteins were identified as potential importin 13 import cargoes for both ratios, another 22 proteins for Imp13/Imp13+Ran and 102 proteins for Imp13/Imp13+Ubc9 (Table S6). Of the 102 proteins identified for Imp13/Imp13+Ubc9, 16 proteins also showed enrichment for Imp13+Ran/Imp13 and consequently were considered to be ambiguous cargoes (Table S7). As importin 13 can bind its export cargo elF1A also in the absence of RanGTP in a non-cooperative manner, some export cargoes are likely also enriched for the 
Imp13/Imp13+Ubc9 ratio, qualifying the majority of the ambiguous proteins as potential export cargoes.

As the established importin 13 export cargo elF1A showed strong enrichment for both the Imp13+Ran/Imp13 and the Imp13+Ran/Imp13+Ubc9 ratio, quantified proteins were considered to be potential export cargoes if enriched for either of these ratios. As the majority of the quantified proteins showed a similar enrichment for both ratios, the mass spectrometry data was analyzed by filtering for Imp13+Ran/Imp13. Using these filtering criteria a total of 113 proteins were identified as potential importin 13 export cargoes, 21 of which had a log2(Imp13+Ran/Imp13) $\geq 3.0$ and 36 of which had a $\log 2$ (Imp13+Ran/Imp13) $\geq 1.5$ (Table S8).

Based on these filtering criteria, the identified cargo candidates were compared with proteins identified in the previous mass spectrometry experiment (3.3.1). In total 66 proteins were detected for both approaches, 19 of which were identified as potential export cargoes and 47 as potential import cargoes (Table S9). The proteins identified in the SILAC screen were also compared with the proteins identified in the importin 13 overexpression screen (see section 3.2). However, there was no overlap between the two screens, except for DBC-1 (KIAA1967), which was identified in one of the three SILAC experiments (Table 12). In this one replicate it was present with a high log2 SILAC ratio of more than two for both the Imp13/Imp13+Ran and the Imp13/Imp13+Ubc9 ratio, implicating it as an importin 13 import and not an export cargo as suggested by the overexpression screen. Finally, the identified proteins were also compared with previous screens for importin 13 cargoes. In a SILAC-based transport (SILAC-Tp) system (76), 309 proteins were identified as potential importin 13 cargoes (using their 2nd-Z-ranking filtering criteria), some of which were validated in bead halo assays but not all. Of these, 28 were also identified in the SILAC screen presented here (Table S10). In a yeast two-hybrid screen for interactors of the testis-specific form of importin 13 (136), 26 proteins were identified, two of which, EIF4G2 and CLASP1, were also identified in this study.

For additional validation of the SILAC screen, several of the quantified proteins were selected for further analysis based on the above filtering criteria and validated using pull-down experiments and importin 13 overexpression experiments (see section 3.3.3.4). 


\subsubsection{Validation of Importin 13 Cargo Candidates Identified in SILAC Screen}

\subsection{Selection of Importin 13 Cargo Candidates for Further Analysis}

Having confirmed that the SILAC screen allowed for the effective identification of known importin 13 substrates, the next step was to verify other identified candidates as importin 13 cargoes. For this, proteins with varying levels of significance were selected, to get an idea with which filtering criteria high confidence importin 13 cargoes can be identified. Export cargoes were grouped into three categories based on their log2 (Imp13+Ran/Imp13) ratio, namely log2 ratios $\geq 0.5, \geq 1.5$ and $\geq 3.0$ (Table 13, Figure 21). For importin 13 import cargoes, proteins were selected based on their Imp13/Imp13+Ran and Imp13/Imp13+Ubc9 ratio, as a high variability between these two groups could be observed (Table 14, Figure 22). Proteins enriched for both or only one of the two ratios and showing different log2 SILAC ratios were chosen. In addition, proteins were selected that showed a good enrichment for both ratios but were only detected in two out of three SILAC experimental replicates.

Additional selection criteria were the size of the proteins $(<100 \mathrm{kDa})$ to facilitate cloning from cDNA as well as available information on function and subcellular location of these proteins. Further, proteins known to form heterodimers or larger complexes were selected, as importin 13 has been reported to mediate the transport of several heterodimers but not their individual subunits $(135,141,146,147)$. 


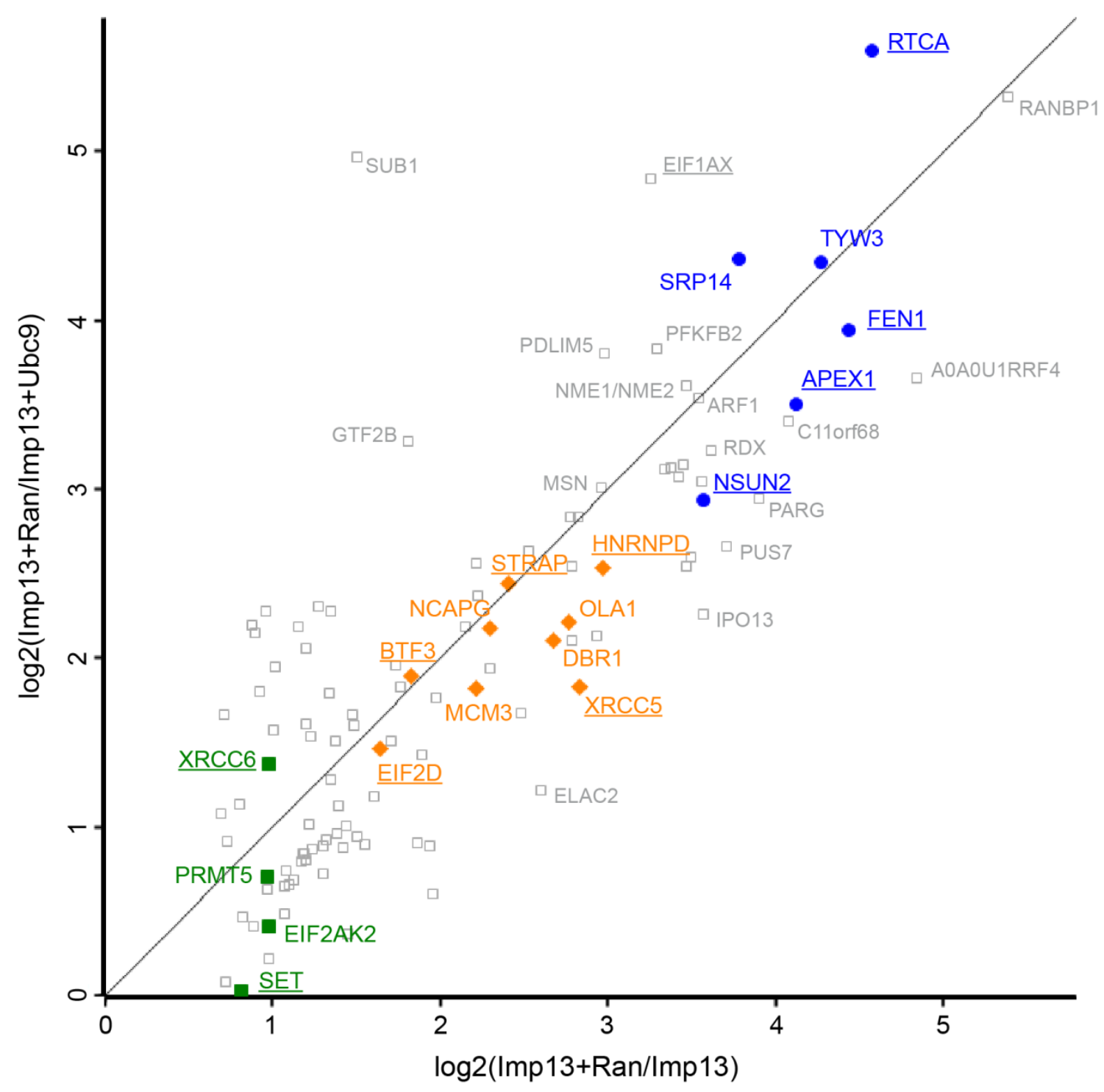

Figure 21: Importin 13 export candidates selected for further validation. All proteins detected by mass spectrometry with a $\log 2(\operatorname{Imp} 13+R a n / I m p 13) \geq 0.5$ (grey squares) for all three biological replicates (3.3.3.1) were plotted as $\log 2(\operatorname{Imp} 13+R a n / I m p 13+U b c 9)$ against $\log 2(\operatorname{Imp} 13+R a n / I m p 13)$ ratios. Proteins highlighted in color were selected for further analysis and underlined proteins were affected by importin 13 overexpression. Blue circles: proteins with an average $\log 2(\operatorname{Imp} 13+$ Ran/Imp13) $\geq 3.0$. Orange diamonds: proteins with an average $\log 2(\operatorname{Imp13+Ran} / \mathrm{Imp13}) \geq 1.5$. Green squares: proteins with an average log2(Imp13+Ran/Imp13) 20.5. Grey squares: importin 13 export candidates that were not analyzed further, high scoring proteins were labeled with their gene name. Note that WDR77, which was selected for further testing is not shown as in two replicates it was present with a $\log 2(\operatorname{Imp} 13+\operatorname{Ran} / \operatorname{Imp} 13)<0.5$. See Table 13 for more details. 
Table 13: List of potential importin 13 export cargoes identified in SILAC screen that were selected for further validation

\begin{tabular}{|c|c|c|c|c|c|c|}
\hline \multicolumn{7}{|c|}{ Potential importin 13 export cargoes } \\
\hline Uniprot ID & Protein Name & Gene & $\log 2(\operatorname{Imp13+Ran} / \operatorname{lm} p 13)$ & $\log 2(\operatorname{Imp} 13+R a n / I m p 13+U b c 9)$ & $-\log (p$ value $)$ & Imp13 pull-down ${ }^{\mathrm{a}}$ \\
\hline \multicolumn{7}{|c|}{ Proteins identified for $\operatorname{Im}$ p13+Ran/Im p13 } \\
\hline \multicolumn{7}{|c|}{$\log 2(\operatorname{Imp} 13+\operatorname{Ran} / \operatorname{Imp} 13) \geq 3$} \\
\hline 000442 & RNA 3-terminal phosphate cyclase & RTCA & 4.57597 & 5.59694 & 2.50708 & - \\
\hline P39748 & Flap endonuclease 1 & FEN1 & 4.43566 & 3.9402 & 1.84603 & - \\
\hline Q6IPR3 & tRNA wybutosine-synthesizing protein 3 homolog & TYW3 & 4.26835 & 4.34712 & 2.42506 & \\
\hline P27695 & DNA-(apurinic or apyrimidinic site) lyase & APEX1 & 4.12269 & 3.50792 & 2.09237 & - \\
\hline P37108 & Signal recognition particle $14 \mathrm{kDa}$ protein & SRP14 & 3.77885 & 4.36023 & 3.05751 & bound with RanGTP \\
\hline Q08J23 & tRNA (cytosine(34)-C(5))-methyltransferase & NSUN2 & 3.56745 & 2.93758 & 2.29027 & - \\
\hline \multicolumn{7}{|c|}{$\log 2(\operatorname{Imp} 13+\operatorname{Ran} / \operatorname{lmp} 13) \geq 1.5$} \\
\hline HOYA96 & Heterogeneous nuclear ribonucleoprotein D0 & HNRNPD & 2.96732 & 2.53505 & 2.24646 & \\
\hline P13010 & X-ray repair cross-complementing protein 5 & XRCC5 & 2.82663 & 1.82907 & 2.72781 & \\
\hline Q9NTK5 & Obg-like ATPase 1 & OLA1 & 2.76445 & 2.21738 & 3.92603 & bound with RanGTP \\
\hline Q9Y3F4 & Serine-threonine kinase receptor-associated protein & STRAP & 2.40696 & 2.44099 & 1.68925 & - \\
\hline Q9BPX3 & Condensin complex subunit 3 & NCAPG & 2.29883 & 2.17429 & 2.48105 & - \\
\hline Q9UK59 & Lariat debranching enzyme & DBR1 & 2.6774 & 2.10752 & 2.66408 & \\
\hline P25205 & DNA replication licensing factor MCM3 & MCM3 & 2.2095 & 1.8214 & 2.44116 & \\
\hline P20290 & Transcription factor BTF3 & BTF3 & 1.82755 & 1.8911 & 1.59095 & \\
\hline P41214 & Eukaryotic translation initiation factor $2 \mathrm{D}$ & EIF2D & 1.63737 & 1.46164 & 1.72077 & \\
\hline \multicolumn{7}{|c|}{$\log 2(\operatorname{Imp} 13+\operatorname{Ran} / \operatorname{lmp} 13) \geq 0.5$} \\
\hline P12956 & X-ray repair cross-complementing protein 6 & XRCC6 & 0.977419 & 1.37652 & 1.40745 & \\
\hline P19525 & Interferon-induced, double-stranded RNA-activated protein kinase & EIF2AK2 & 0.97668 & 0.410077 & 2.54683 & \\
\hline 014744 & Protein arginine $\mathrm{N}$-methyltransferase 5 & PRMT5 & 0.967085 & 0.708434 & 1.96941 & \\
\hline Q9BQA1 & Methylosome protein 50 & WDR77 & 0.946335 & 0.381559 & 1.04849 & bound with RanGTP \\
\hline Q01105 & Protein SET & SET & 0.81555 & 0.0277848 & 2.56819 & \\
\hline \multicolumn{7}{|c|}{ Export control } \\
\hline P47813 & Eukaryotic translation initiation factor $1 \mathrm{~A}$ & EIF1A & 3.25237 & 4.8423 & 4.24736 & bound w ith RanGTP \\
\hline
\end{tabular}

a: Protein binding from digitonin HeLa P4 cell lysate to Hzz-importin 13 immobilized on IgG-Sepharose

-: tested in importin13 pull-down but no binding detected 


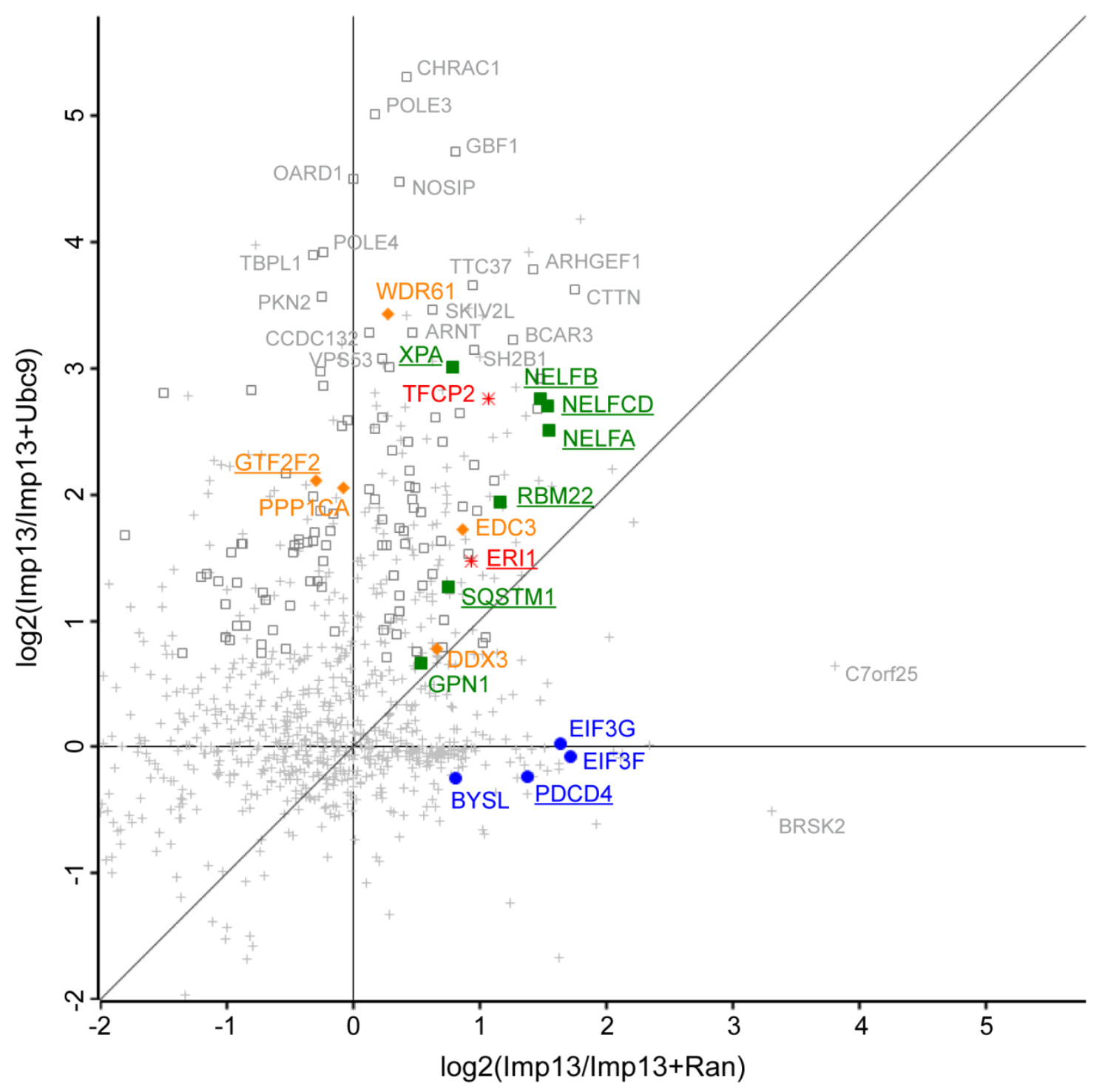

Figure 22: Importin 13 import candidates selected for further validation All proteins detected by mass spectrometry (grey cross) in the triple SILAC experiment (3.3.3.1) were plotted as $\log 2(I \mathrm{mp} 13 / \mathrm{Imp} 13+U b c 9)$ against $\log 2(\mathrm{Imp} 13 / \mathrm{Imp13+Ran})$ ratios. Ratios were averaged from three SILAC biological replicate experiments. Proteins highlighted in color were selected for further analysis and underlined proteins were affected by importin 13 overexpression. Red stars: proteins enriched for Imp13/Imp13+Ran and Imp13/Imp13+Ubc9. Blue circles: proteins enriched only for Imp13/Imp13+Ran. Orange diamonds: proteins enriched only for Imp13/Imp13+Ubc9. Green squares: proteins enriched for Imp13/Imp13+Ran and Imp13/Imp13+Ubc9 in two out of three SILAC replicates. Grey squares: proteins detected with a $\log 2(\operatorname{Imp} 13 / \operatorname{Imp} 13+U b c 9) \geq 0.5$ for all three SILAC biological replicates. See Table 14 for more details. 
Table 14: List of potential importin 13 import cargoes identified in SILAC screen that were selected for further validation

\begin{tabular}{|c|c|c|c|c|c|c|}
\hline \multicolumn{7}{|c|}{ Potential importin 13 import cargoes } \\
\hline Uniprot ID & Protein Name & Gene & $\log 2(\operatorname{Imp} 13 / \operatorname{lm} p 13+\operatorname{Ran})$ & Jbc9) & $-\log (p$ value $)$ & Imp13 pull-down ${ }^{a}$ \\
\hline \multicolumn{7}{|c|}{ Proteins identified for Imp13/Im p13+Ran \& Im p13/Im p13+Ubc9 } \\
\hline Q12800 & Alpha-globin transcription factor CP2 & TFCP2 & 1.06516 & 2.75921 & 2.5538 & \\
\hline Q8IV48 & 3-5 exoribonuclease 1 & ERI1 & 0.93069 & 1.46739 & 3.46771 & reduced by Ubc9 \& RanGTP \\
\hline \multicolumn{7}{|c|}{ Proteins identified for Im p13/Imp13+Ran } \\
\hline 000303 & Eukaryotic translation initiation factor 3 subunit $F$ & EF3F & 1.71919 & -0.0768488 & 1.84627 & \\
\hline 075821 & Eukaryotic translation initiation factor 3 subunit G & EF3G & 1.63573 & 0.0269878 & 1.74379 & \\
\hline Q53EL6 & Programmed cell death protein 4 & PDCD4 & 1.38179 & -0.239508 & 1.9684 & \\
\hline Q13895 & Bystin & BYSL & 0.812382 & -0.246966 & 2.6437 & \\
\hline \multicolumn{7}{|c|}{ Proteins identified for Imp13/Imp13+Ubc9 } \\
\hline Q9GZS3 & WD repeat-containing protein 61 & WDR61 & 0.269134 & 3.43407 & 2.461 & reduced by Ubc9 \& RanGTP \\
\hline P13984 & General transcription factor IIF subunit 2 & GTF2F2 & -0.29533 & 2.1155 & 2.8392 & \\
\hline P62136 & Serine/threonine-protein phosphatase PP1-alpha catalytic subunit & PPP1CA & -0.0792858 & 2.05476 & 2.28248 & \\
\hline Q96F86 & Enhancer of mRNA-decapping protein 3 & EDC3 & 0.867101 & 1.72773 & 2.35559 & reduced by Ubc9 \& RanGTP \\
\hline 000571 & ATP-dependent RNA helicase DDX3X/DDX3Y & DDX3 & 0.664858 & 0.780721 & 1.90872 & \\
\hline \multicolumn{7}{|c|}{ Proteins identified for $\operatorname{Im}$ p13/Imp13+Ran \& Imp13/Imp13+Ubc9 for two out of three SILAC experim ents } \\
\hline F2Z2T2 & DNA repair protein complementing XP-A cells & XPA & 0.783184 & 3.01102 & 1.51456 & \\
\hline Q9HCN4 & GPN-loop GTPase 1 & GPN1 & 0.535254 & 0.668491 & 2.6656 & \\
\hline Q13501 & Sequestosome-1 & SQSTM1 & 0.755231 & 1.26888 & 2.83389 & \\
\hline Q9NW64 & Pre-mRNA-splicing factor RBM22 & RBM22 & 1.15759 & 1.93592 & 2.33561 & \\
\hline Q9H3P2 & Negative elongation factor $A$ & NELFA & 1.54779 & 2.50404 & 2.54973 & \\
\hline Q8WX92 & Negative elongation factor $B$ & NELFB & 1.47736 & 2.75735 & 2.2666 & \\
\hline Q81XH7 & Negative elongation factor $\mathrm{C} / \mathrm{D}$ & NELFCD & 1.54033 & 2.70383 & 2.29508 & \\
\hline \multicolumn{7}{|c|}{ Import control } \\
\hline P63279 & SUMO-conjugating enzyme UBC9 & UBE2I & $-3.65595^{*}$ & $1.46848^{*}$ & $0.480393^{*}$ & reduced by RanGTP \\
\hline
\end{tabular}

a: Protein binding from digitonin HeLa P4 cell lysate to Hzz-importin 13 immobilized on IgG-Sepharose

*: recombinant unlabeled Ubc9 was added to the "light" cell extract, resulting in a not meaningful ratio 


\subsection{Validation of Importin 13 Cargo Candidates Using Pull-down Experiments}

As the identified importin 13 cargo candidates were differently regulated by Ubc 9 or RanGTP $_{\mathrm{Q} 69 \mathrm{~L}}$, the binding experiments were repeated without SILAC and analyzed by immunoblotting using commercially available antibodies. To this end, Hzz-importin 13 was immobilized on IgG-Sepharose and incubated with unlabeled digitonin HeLa P4 cell lysate in the absence or presence of Ubc9 or RanGTP $\mathrm{Q}_{\mathrm{QL}}$. Beads were not pooled as in the SILAC experiment but instead bound proteins were eluted separately with magnesium chloride. Eluates were separated by SDS-PAGE and immunoblotted with antibodies targeted against the potential importin 13 substrates (Figure 23). The known importin 13 export cargo elF1A was used as a control and indeed elF1A bound in the presence of RanGTP $_{\text {Q69L }}$ but not Ubc9 and showed reduced binding in the absence of both.

Not for all proteins analyzed binding to immobilized importin 13 could be detected. This could be due to unspecific antibodies, low endogenous protein levels or proportionally less protein binding to importin 13 compared to the other proteins. Of seven export cargo candidates (APEX1, FEN1, STRAP, NSUN2, WDR77, OLA1, SRP14) tested, OLA1, WDR77 and SRP14 were bound to Hzz-importin 13 and as expected for bona fide export cargoes binding of OLA1 and WDR77 only occurred in the presence of RanGTP ${ }_{\text {Q69L. }}$. SRP14, similarly to eIF1A, showed binding to importin 13 both in the presence and absence of RanGTP ${ }_{\mathrm{Q} 69 \mathrm{~L}}$ but not in the presence of Ubc9. All import cargoes tested, namely ERI1, WDR61 and EDC3 bound to importin 13 and binding was abolished in the presence of Ubc9 or RanGTP ${ }_{\mathrm{Q} 69 \mathrm{~L}}$.

In summary, OLA1, WDR77 and SRP14 bound to importin 13 in the presence of RanGTP, validating them as potential export cargoes, while ERI1, WDR61 and EDC3 bound to importin 13 only in the absence of Ubc9 or RanGTP ${ }_{\mathrm{Q} 69 \mathrm{~L}}$, validating them as potential import cargoes. 

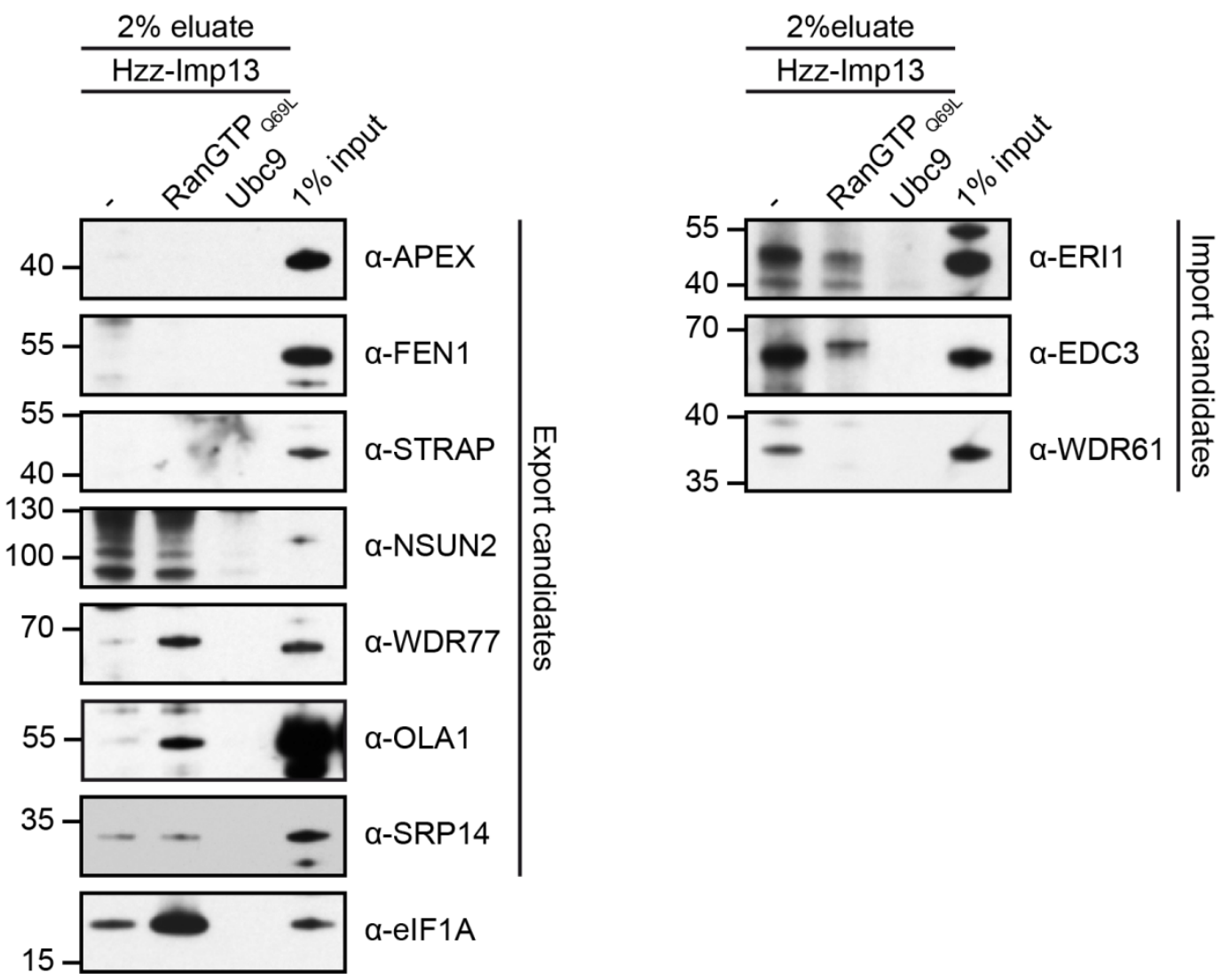

Figure 23: Endogenous SILAC candidate proteins that bind to importin 13 from HeLa P4 cell extract in a RanGTP ${ }_{\mathrm{Q} 69 \mathrm{~L}}$ or Ubc9 dependent manner. Hzz-tagged importin 13 (0.5 nmol) was immobilized on IgG-Sepharose and incubated with a digitonin HeLa P4 cell extract precleared with Hzz/lgG-Sepharose and phenyl-Sepharose in the absence or presence of $10 \mu \mathrm{M}$ RanGTP Q69L $_{\text {or }}$ $5 \mu \mathrm{M}$ Ubc9 in transport buffer. Bound proteins were eluted by magnesium chloride elution, separated by SDS-PAGE and analyzed by immunoblotting.

\subsection{Validation of Importin 13 Cargo Candidates in Overexpression Experiments}

For further validation of the SILAC screen, the identified importin 13 cargo candidates were tested in importin 13 overexpression experiments. For this, 36 cargo candidates were cloned as C-terminal HA-fusion constructs and 34 as N-terminal GFP-GST-fusion constructs. Cargoes were fused to a GFP-GST-tag to significantly increase their size and prevent their passive diffusion through the NPC. Generated plasmids were expressed in HeLa P4 cells and analyzed for any changes in subcellular localization upon coexpression of FLAG-importin 13. GFP-GST-Ubc9 and elF1A-GFP were included as positive controls (see section 3.1.3), while dGFP-GST-cNLS was included as a negative control (see section 3.2.1) of importin 13 mediated transport. 
Of 18 import cargo candidates tested (Figure 24 (HA-tagged), Figure 25 (GFP-GST-tagged), Table 15), 6 proteins, namely BYSL, PDCD4, GTF2F2, XPA, RBM22 and NELFA, fused to an HA-tag already exhibited a nuclear localization in the absence of importin 13, precluding the identification of an importin 13 mediated nuclear localization. Proteins from all four filtering groups (Imp13/Imp13+Ran, Imp13/Imp13+Ubc9, as well as both ratios with proteins identified in two or three of the SILAC experiments) were affected by importin 13 overexpression. ERI1 and NELFCD, which showed a strong enrichment for the Imp13/Imp13+Ran and the Imp13/Imp13+Ubc9 ratio, were both relocalized to the nucleus if importin 13 was coexpressed, validating them as import cargo candidates. Surprisingly, PDCD4, WDR61, GTF2F2, XPA, SQSTM1 (only in the presence of LMB, details follow below), RBM22 and NELFA were not relocalized to the nucleus upon importin 13 coexpression but instead to the cytoplasm. Of these, GTF2F2 showed a slight enrichment for the Imp13+Ran/Imp13 ratio designating it as a possible importin 13 export cargo. As mentioned earlier, the export cargo elF1A has been demonstrated to bind effectively to importin 13 also in the absence of RanGTP, thus cargo candidates enriched for the Imp13/Imp13+Ubc9 ratio could also correspond to export cargo candidates. For the other proteins, importin 13 could function as a negative regulator of nuclear import, as reported for the testis-specific form of importin 13 (136), or an additional factor or modification is required to facilitate their importin 13-dependent nuclear import. Note that some of the proteins were only affected by importin 13 overexpression if fused to either the HA- or the GFP-GST-tag, suggesting that the fusion tag size and position can affect the interaction with importin 13. Observed importin 13 effects were independent of the FLAG-fusion tag, as untagged importin 13 had the same effect on the subcellular localization of both import and export cargo candidates as FLAG-tagged importin 13 (Figure S5). 


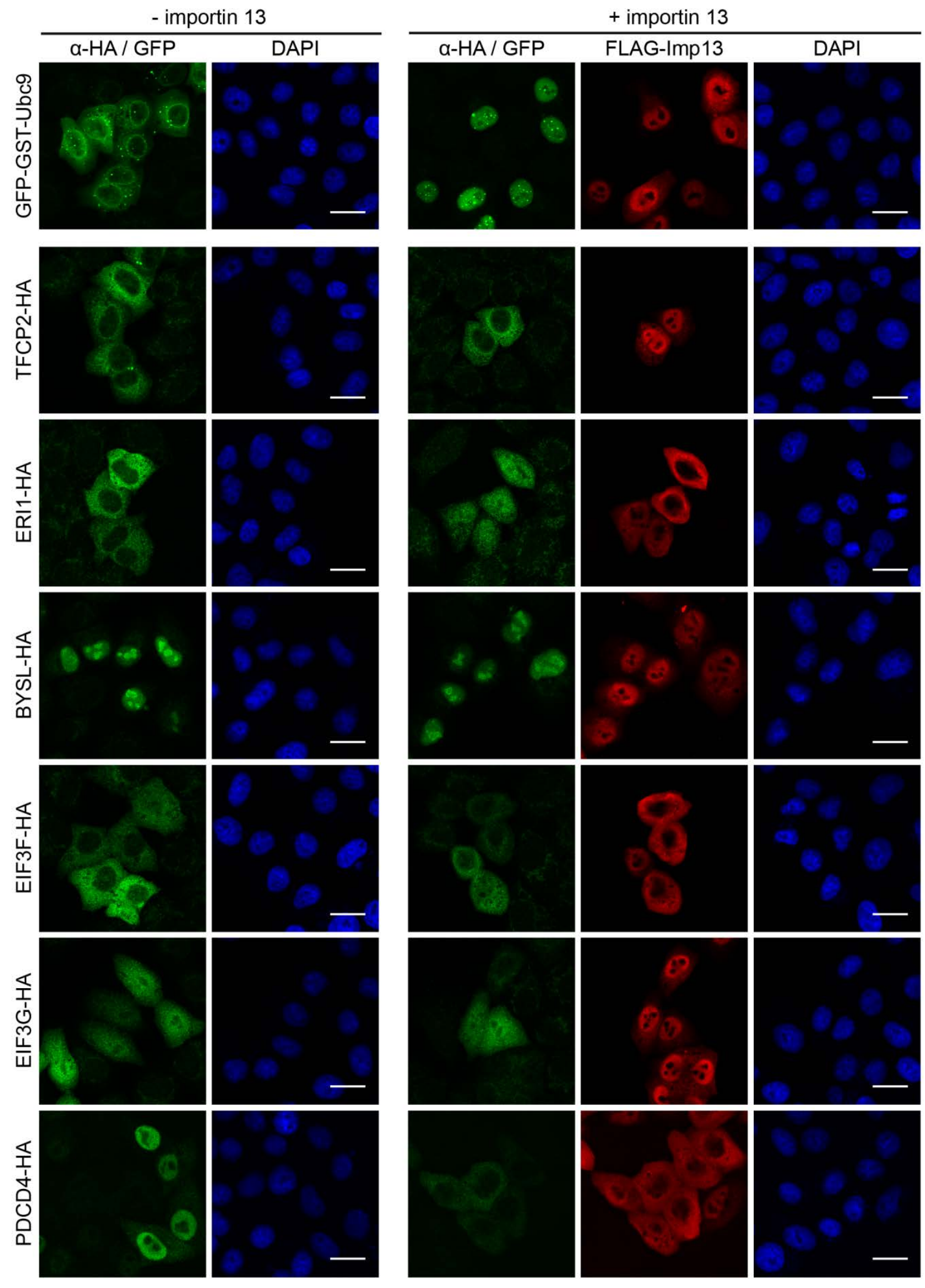




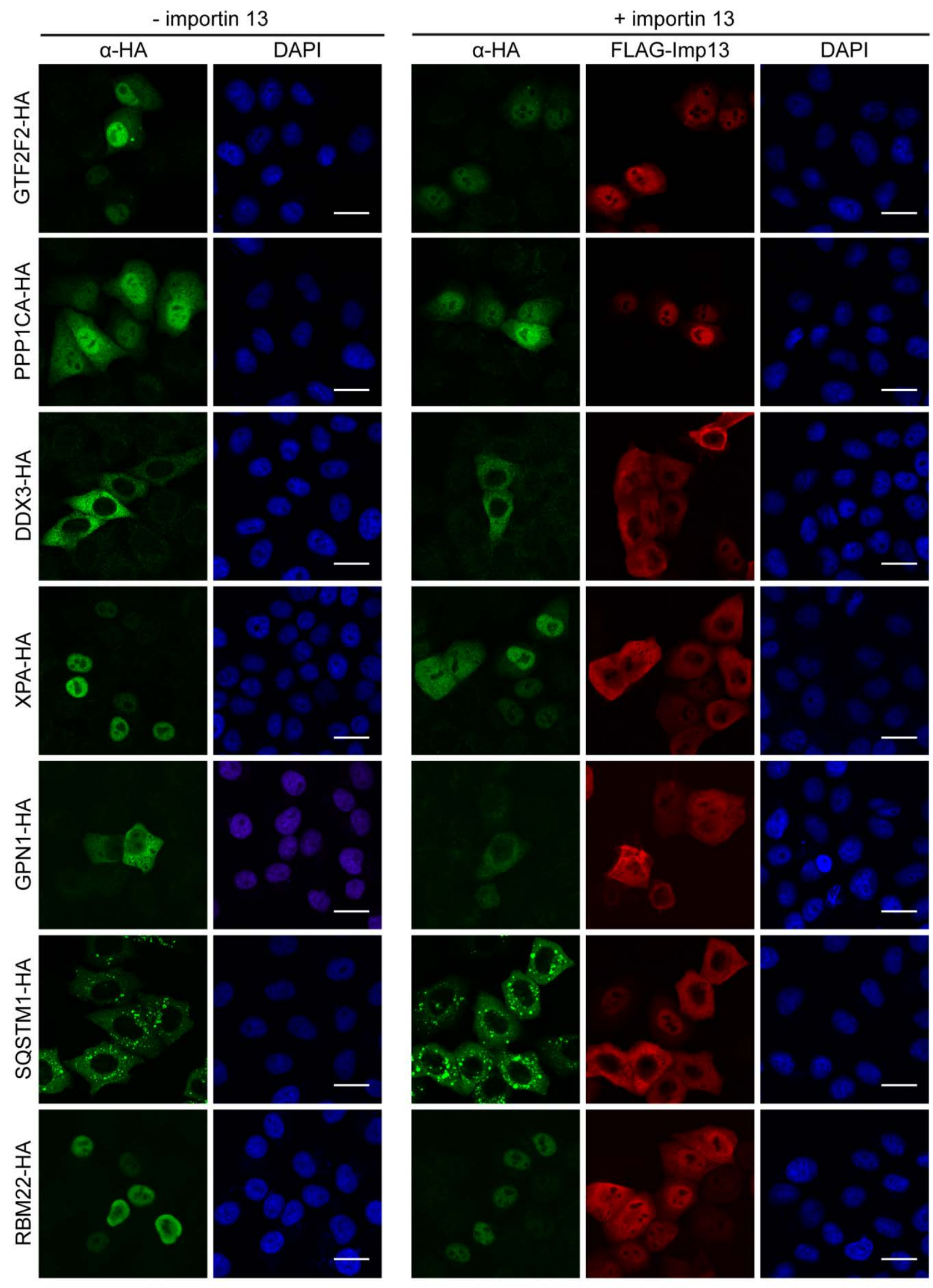



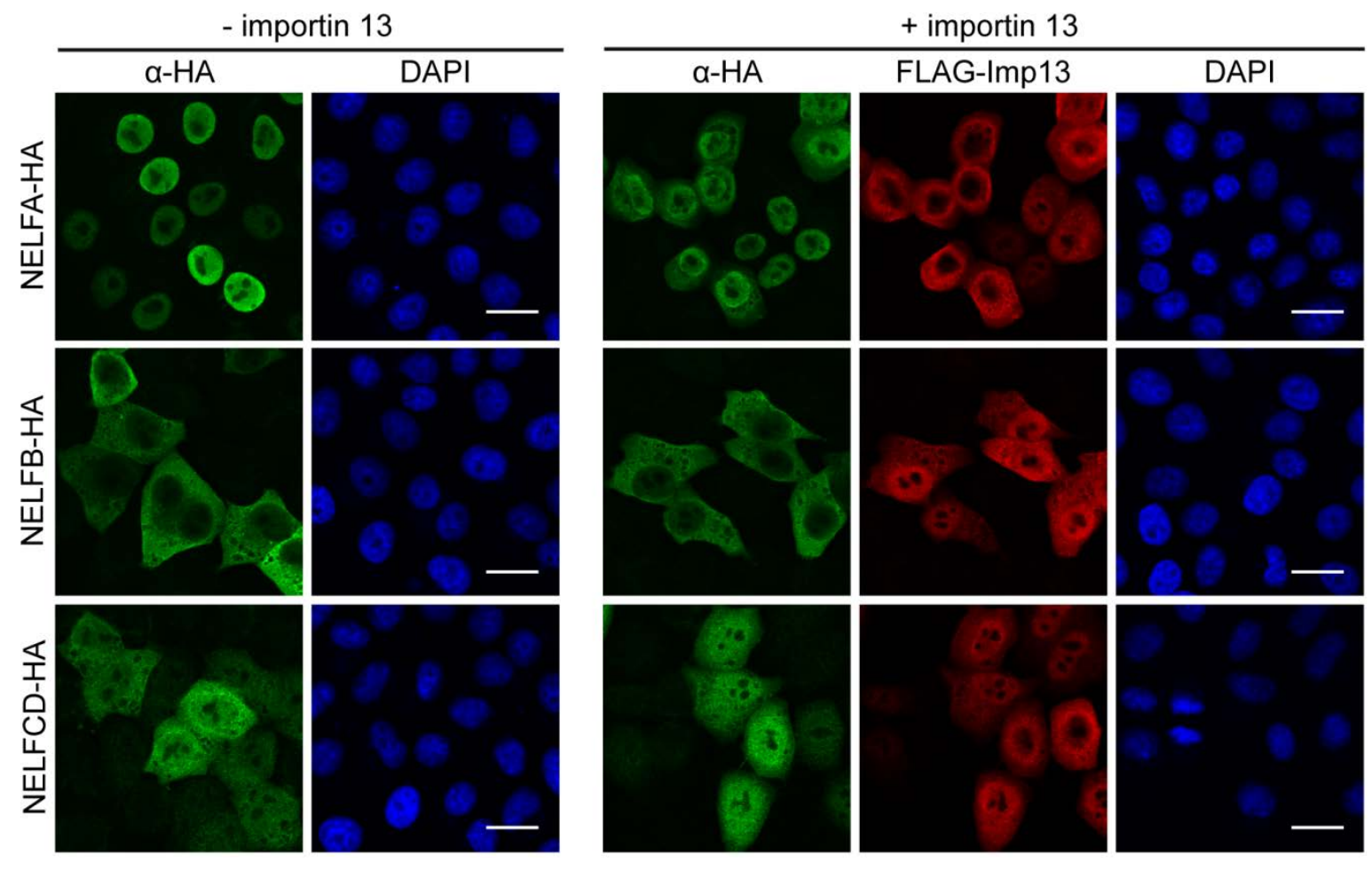

Figure 24: Effect of FLAG-importin 13 overexpression on subcellular localization of HA-tagged SILAC import cargo candidates. HeLa P4 cells were transiently cotransfected with plasmids coding for HA-tagged proteins form the SILAC screen and FLAG-importin 13 or an empty control vector (pcDNA3.1-HA) using the calcium phosphate method. FLAG-importin 13 and HA-substrates were visualized by indirect immunofluorescence with an anti-FLAG and anti-HA antibody, respectively. See Table 15 for summary of importin 13 effect. The scale bars correspond to $20 \mu \mathrm{m}$. DDX3, XPA and SQSTM1 were tested by Christiane Spillner. 

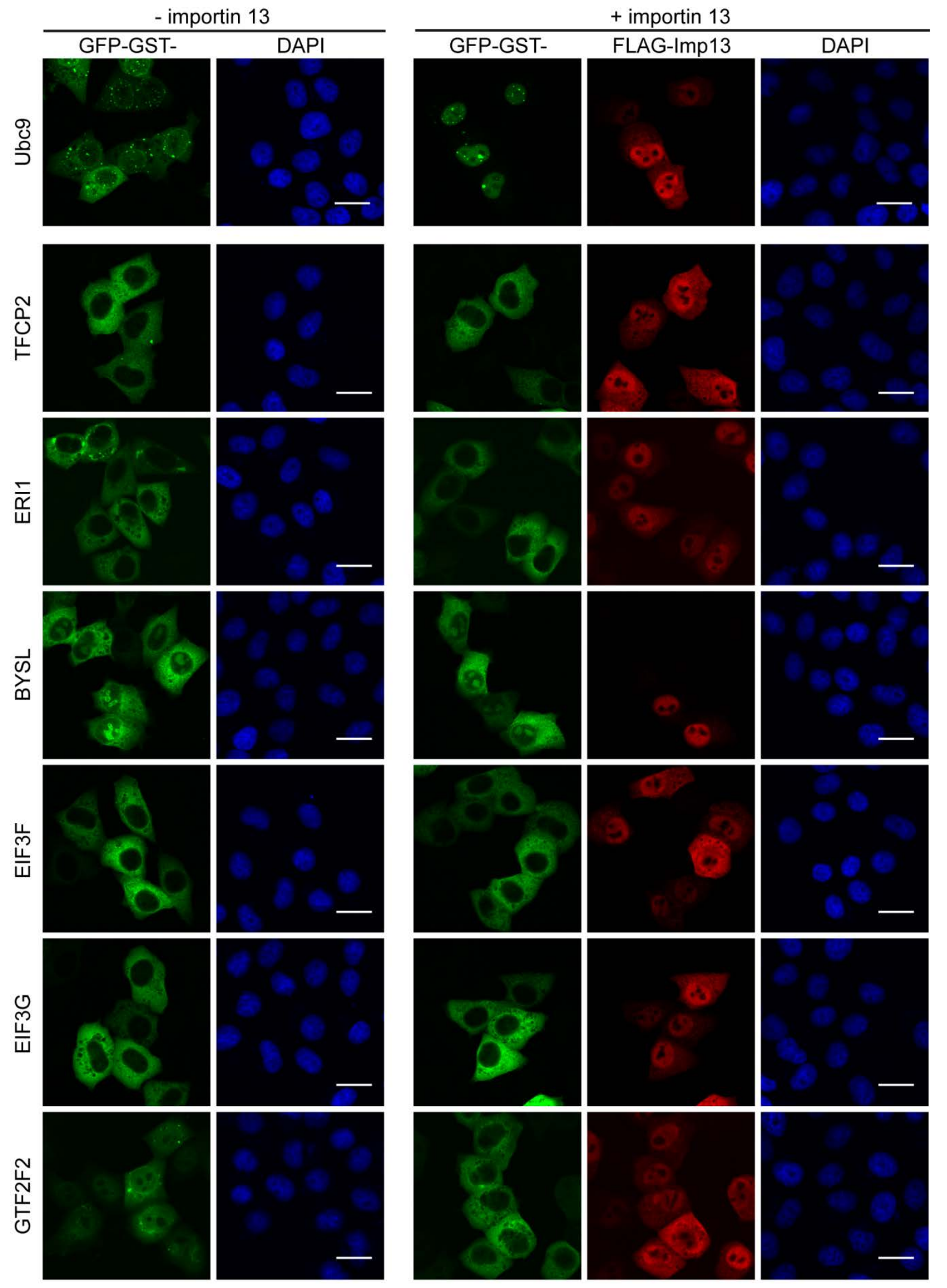


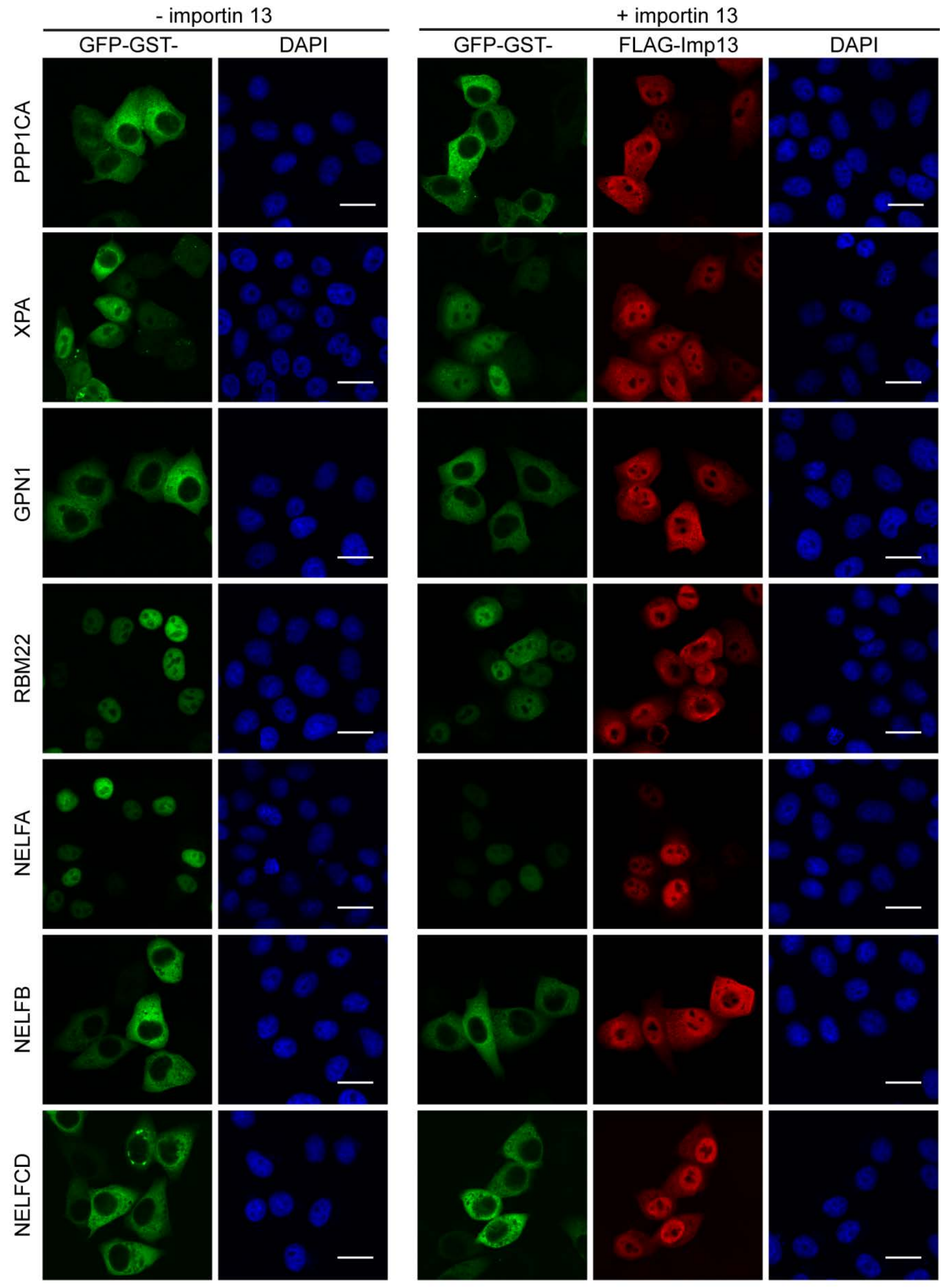


Figure 25: Effect of FLAG-importin 13 overexpression on subcellular localization of GFP-GST-tagged SILAC import cargo candidates. HeLa P4 cells were transiently cotransfected with plasmids coding for GFP-GST-tagged proteins form the SILAC screen and FLAG-importin 13 or an empty control vector (pEGFP-GST) using the calcium phosphate method. FLAG-importin 13 was visualized by indirect immunofluorescence with an anti-FLAG antibody. See Table 15 for summary of importin 13 effect. The scale bars correspond to $20 \mu \mathrm{m}$. XPA and RBM22 were tested by Christiane Spillner. 
Table 15: Effect of importin 13 overexpression on importin 13 import cargo candidates identified in SILAC screen ${ }^{\#}$

\begin{tabular}{|c|c|c|c|c|c|c|}
\hline \multicolumn{7}{|c|}{ Potential importin 13 import cargoes } \\
\hline \multicolumn{7}{|c|}{ Proteins identified for Imp13/Im p13+Ran \& Im p13/Im p13+Ubc9 } \\
\hline Q12800 & Alpha-globin transcription factor CP2 & TFCP2 & $x$ & & $\mathbf{x}$ & $\mathbf{x}$ \\
\hline Q8IV48 & 3-5 exoribonuclease 1 & ERI1 & $\sqrt{ }$ & & $x$ & $\sqrt{ }$ \\
\hline \multicolumn{7}{|c|}{ Proteins identified for Im p13/Im p13+Ran } \\
\hline 000303 & Eukaryotic translation initiation factor 3 subunit $F$ & EF3F & $x$ & & $x$ & $x$ \\
\hline 075821 & Eukaryotic translation initiation factor 3 subunit G & EF3G & $x$ & & $x$ & $\mathbf{x}$ \\
\hline Q53EL6 & Programmed cell death protein 4 & PDCD4 & $\sqrt{ } \mathscr{V}^{*}$ & & & \\
\hline Q13895 & Bystin & BYSL & $\mathbf{x}$ & & $x$ & $\mathbf{x}$ \\
\hline \multicolumn{7}{|c|}{ Proteins identified for Imp13/Im p13+Ubc9 } \\
\hline Q9GZS3 & WD repeat-containing protein 61 & WDR61 & $\iota^{*}$ & & $x$ & \\
\hline P13984 & General transcription factor IIF subunit 2 & GTF2F2 & $x$ & & $\checkmark \checkmark^{*}$ & \\
\hline P62136 & Serine/threonine-protein phosphatase PP1-alpha catalytic subunit & PPP1CA & $x$ & & $x$ & $\mathbf{x}$ \\
\hline Q96F86 & Enhancer of mRNA-decapping protein 3 & EDC3 & $x$ & & $\mathbf{x}$ & $\mathbf{x}$ \\
\hline 000571 & ATP-dependent RNA helicase DDX3X/DDX3Y & DDX3 & $x$ & $x$ & & $x$ \\
\hline \multicolumn{7}{|c|}{ Proteins identified for $\operatorname{Im}$ p13/Imp13+Ran \& Imp13/Imp13+Ubc9 for two out of three SILAC experiments } \\
\hline F2Z2T2 & DNA repair protein complementing XP-A cells & XPA & $\sqrt{ }^{*}$ & & $x$ & \\
\hline Q9HCN4 & GPN-loop GTPase 1 & GPN1 & $x$ & & $x$ & $x$ \\
\hline Q13501 & Sequestosome-1 & SQSTM1 & $x$ & $\checkmark V^{*}$ & $x$ & $\checkmark$ \\
\hline Q9NW64 & Pre-mRNA-splicing factor RBM22 & RBM22 & $x$ & & $\checkmark$ & $\checkmark$ \\
\hline Q9H3P2 & Negative elongation factor A & NELFA & $\checkmark$ & & $x$ & \\
\hline Q8WX92 & Negative elongation factor B & NELFB & $x$ & & $x$ & $x$ \\
\hline Q8IXH7 & Negative elongation factor $C / D$ & NELFCD & $\checkmark$ & & $x$ & $\checkmark$ \\
\hline \multicolumn{7}{|c|}{ Import control } \\
\hline P63279 & SUMO-conjugating enzyme UBC9 & UBE2I & $x$ & & $\checkmark \checkmark$ & $\checkmark \checkmark$ \\
\hline
\end{tabular}

\#: two green ticks: strong importin 13 effect; one green tick: weak importin 13 effect; black cross: no importin13 effect

*: protein redistributed to cytoplasm not nucleus

**: cells were treated with $10 \mathrm{nM} \mathrm{LMB}$ for 2 hours, to shift the cytoplasmic protein to the nucleus 
For the export cargo candidates, proteins from all three filtering criteria $(\log 2(\operatorname{Imp} 13+R a n / I m p 13) \geq 3.0, \geq 1.5$ and $\geq 0.5)$ could be validated as importin 13 cargoes (Figure 26, Figure 27, Table 16). Of 19 proteins tested, 9 localized to the cytoplasm and WDR77 to the nucleus if importin 13 was coexpressed. For the filtering criterion $\log 2(\operatorname{Imp} 13+R a n / I m p 13) \geq 0.5 \leq 1.5$, XRCC6 and SET were identified to be affected by importin 13 coexpression, whereas for the log2(Imp13+Ran/Imp13) $\geq 1.5 \leq 3.0$, HNRNPD, STRAP, BTF3 and EIF2D were affected (see Figure 28 for BTF3 and EIF2D). In contrast, all proteins validated as $\log 2(\operatorname{Imp} 13+\operatorname{Ran} / \mathrm{Imp} 13) \geq 3.0$, namely RTCA, FEN1, APEX1 and NSUN2, could be validated as importin 13 cargo candidates, with the exception of SRP14 and TYW3. However, as overexpressed TYW3 already has a cytoplasmic localization in the absence of importin 13 coexpression, an involvement of importin 13 in the subcellular localization of TYW3 cannot be confirmed with this assay. Similarly, EIF2AK2, BTF3, PRMT5 and EIF2D were also cytoplasmic and could not be analyzed for an importin 13 effect. The observed increased nuclear localization of WDR77 in the presence of overexpressed importin 13 was surprising, especially as in the previous binding experiment it was shown to bind exclusively in the presence of RanGTP ${ }_{\mathrm{Q} 69 \mathrm{~L}}$ (3.3.3.4.2). Possibly, WDR77 requires another factor apart from RanGTP to be exported by importin 13. 


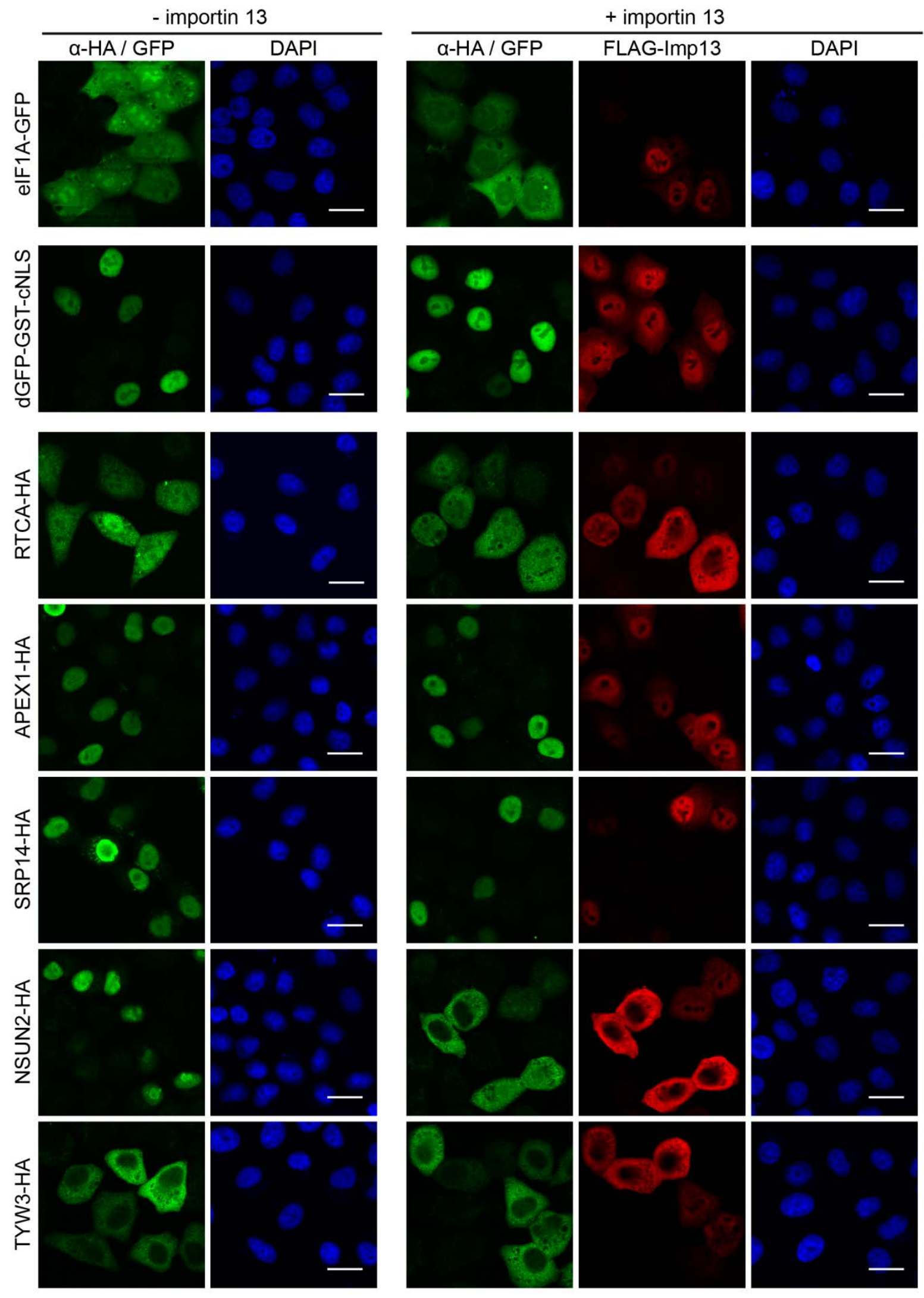




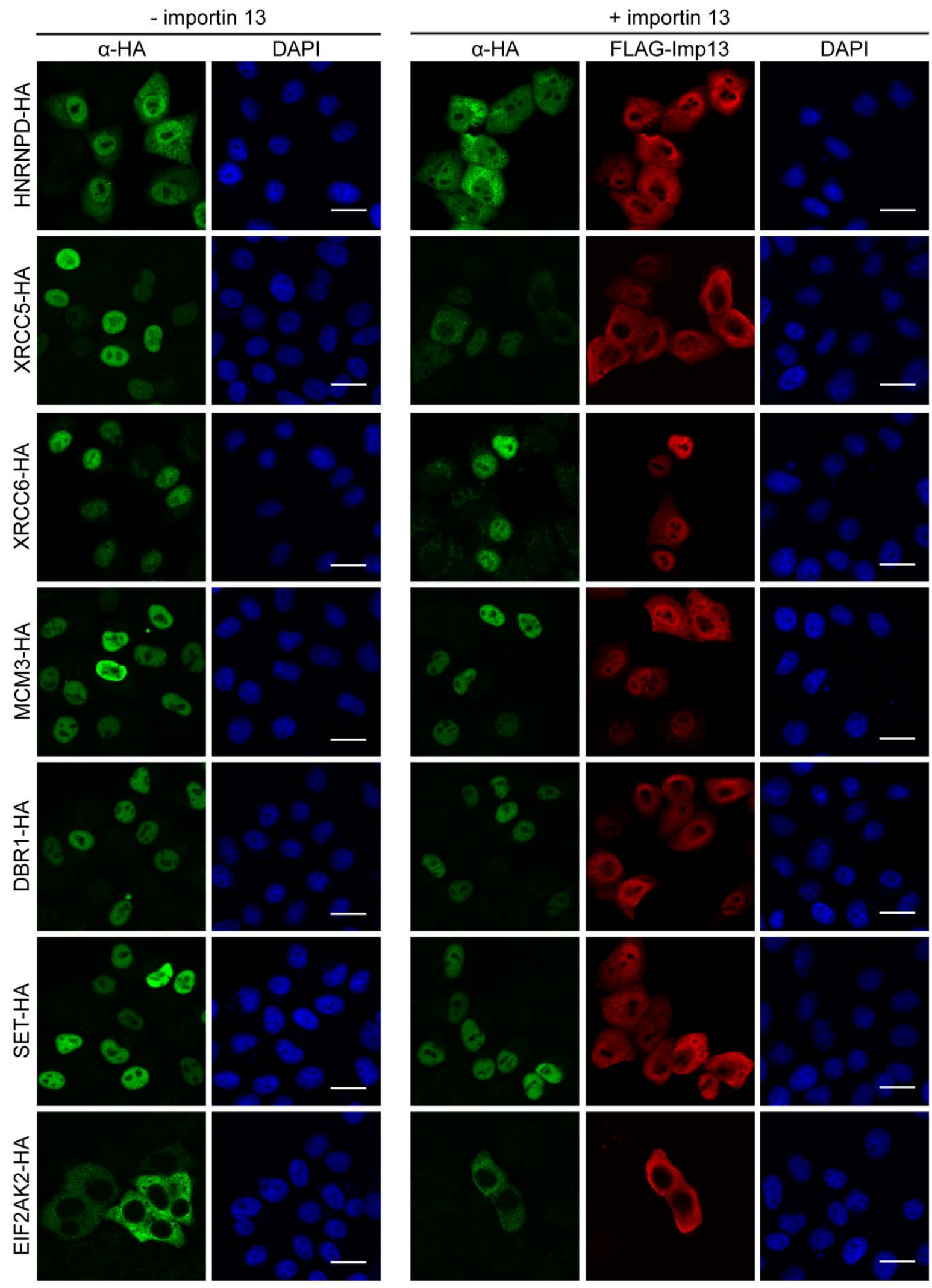



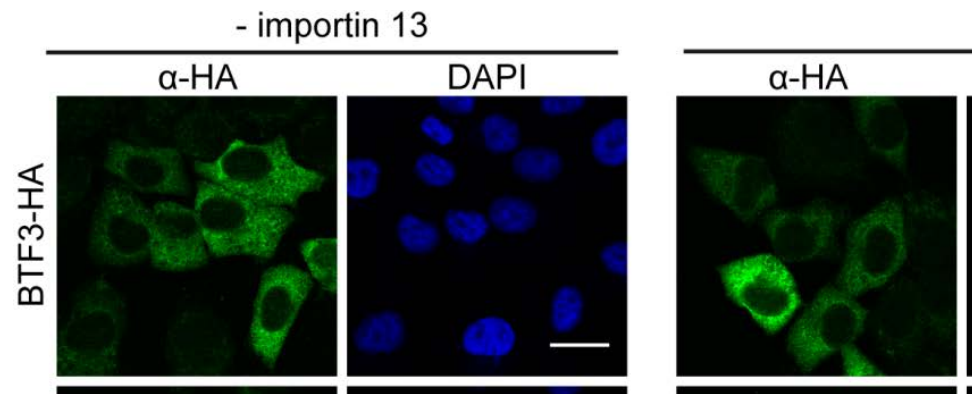

+ importin 13
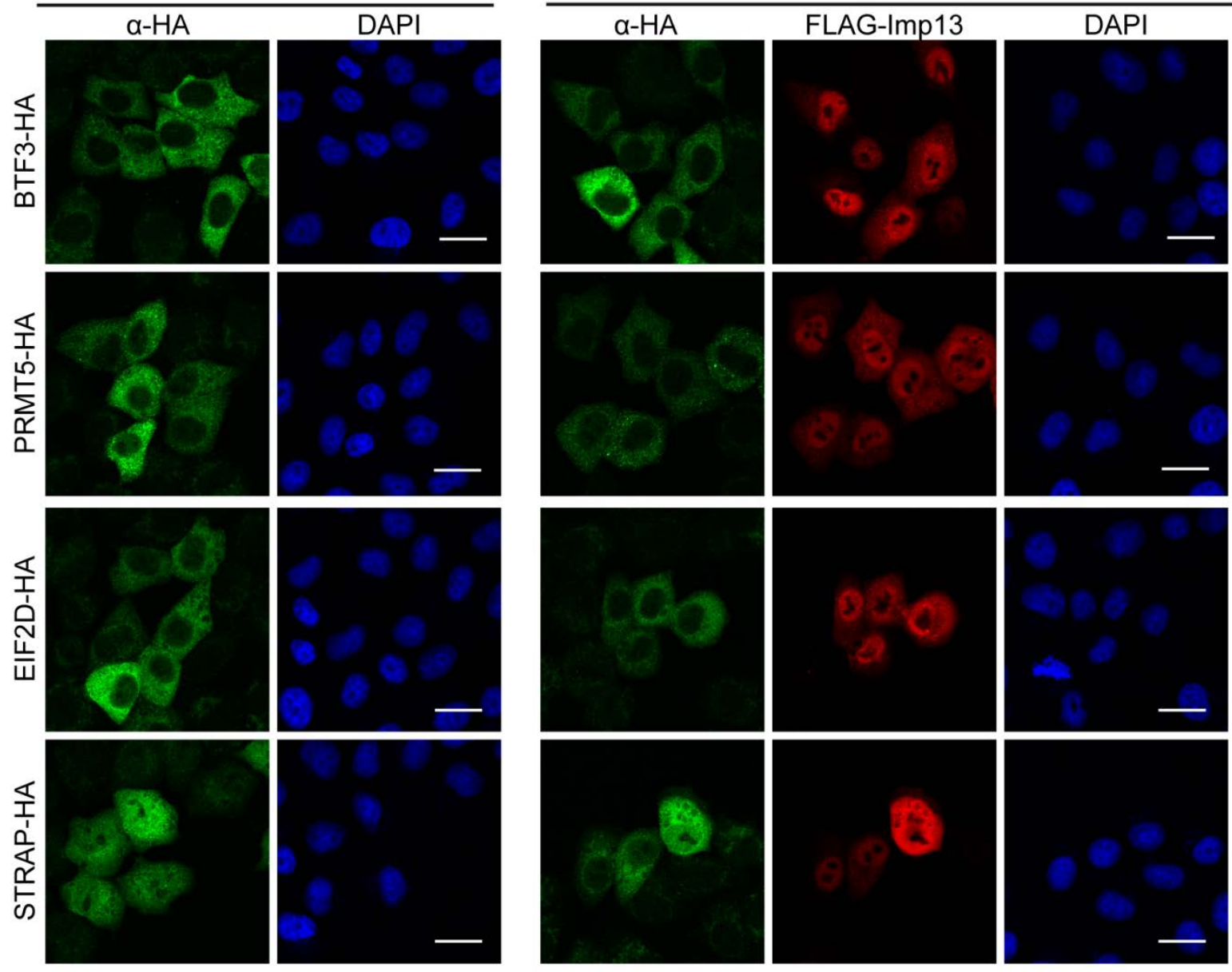

Figure 26: Effect of FLAG-importin 13 overexpression on subcellular localization of HA-tagged SILAC export cargo candidates. HeLa P4 cells were transiently cotransfected with plasmids coding for HA-tagged proteins from the SILAC screen and FLAG-importin 13 or an empty control vector (pcDNA3.1-HA) using the calcium phosphate method. FLAG-importin 13 and HA-substrates were visualized by indirect immunofluorescence with an anti-FLAG and anti-HA antibody, respectively. See Table 16 for summary of importin 13 effect. The scale bars correspond to $20 \mu \mathrm{m}$. XRCC5 and EIF2AK2 were tested by Christiane Spillner. 

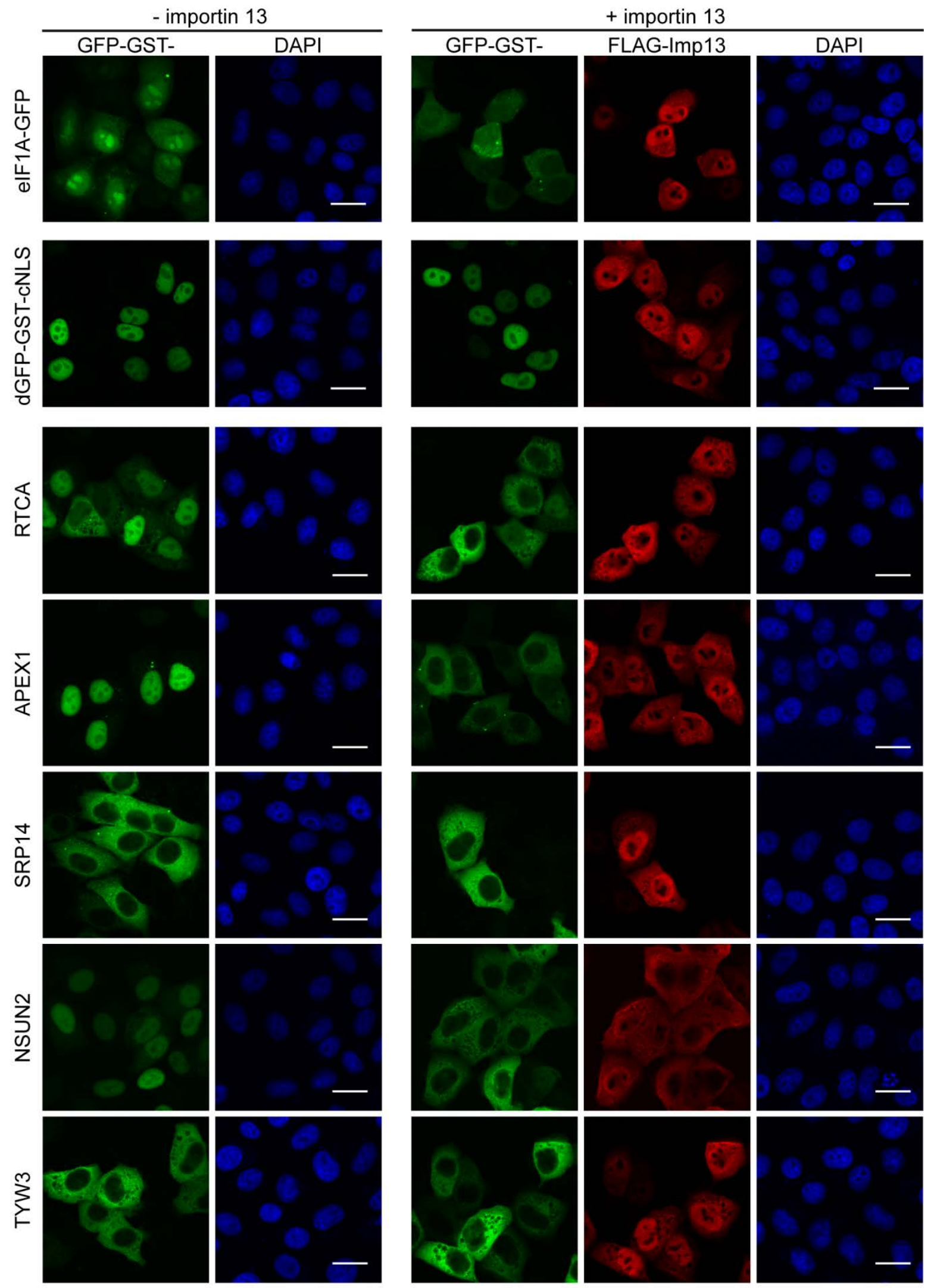


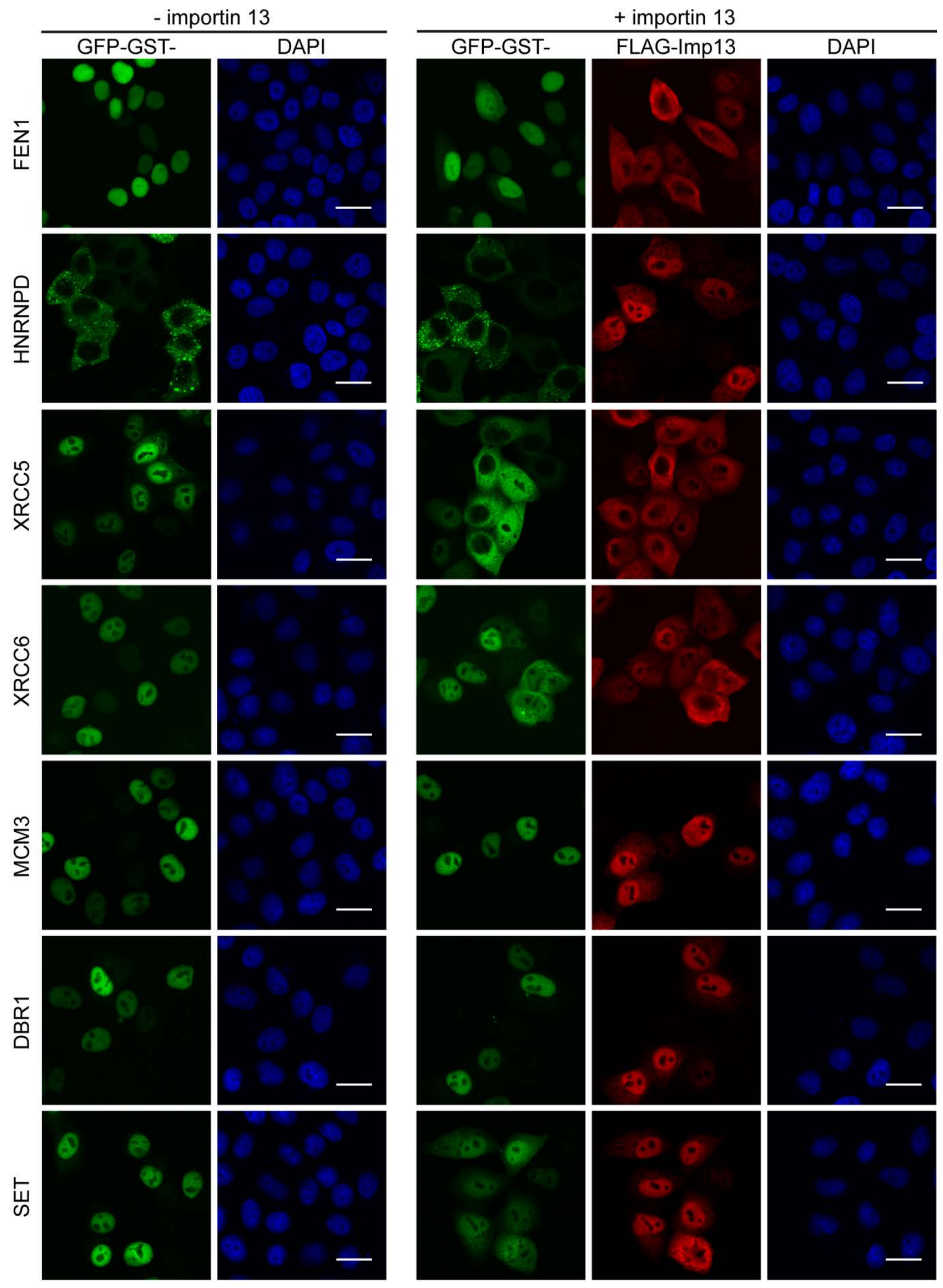



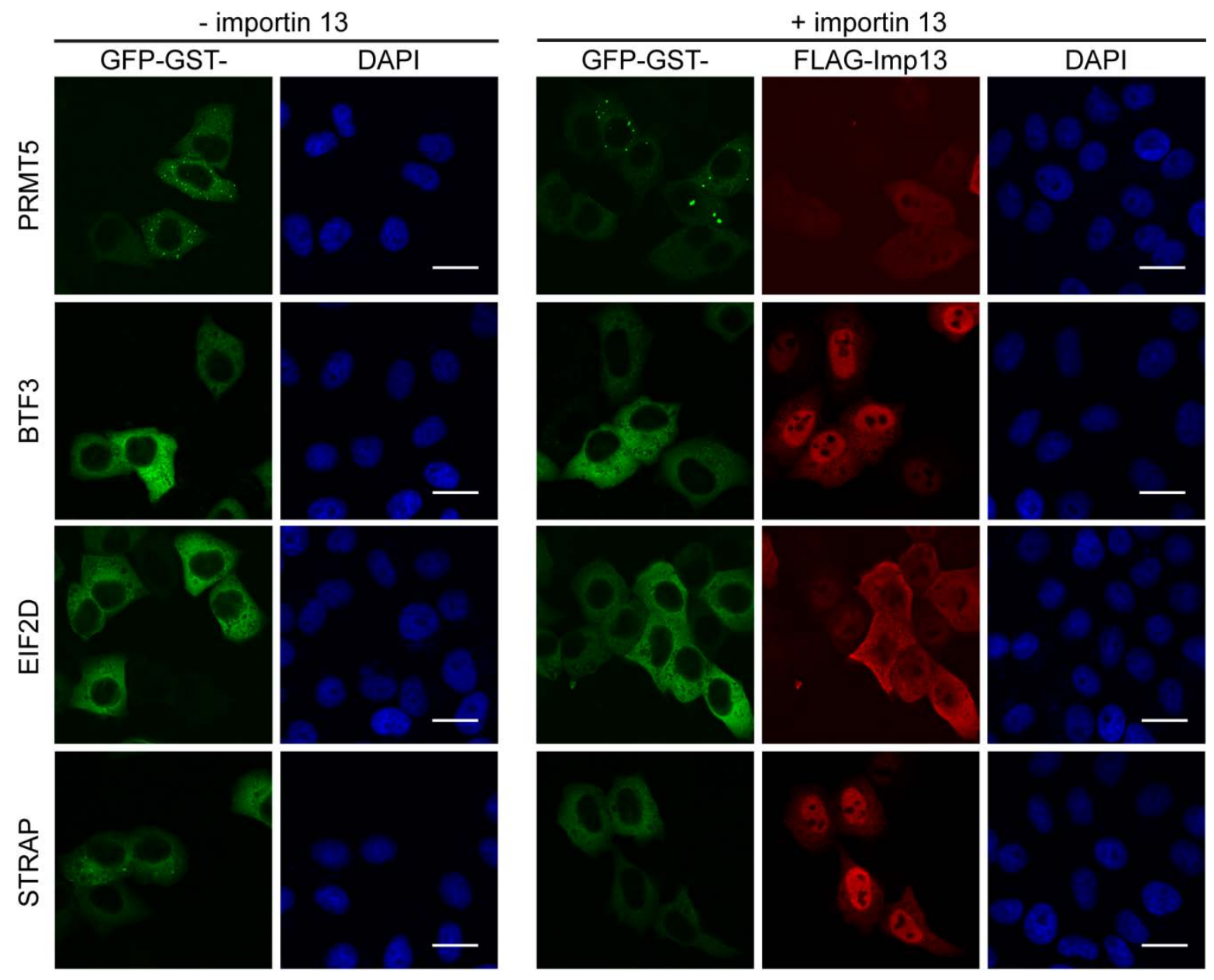

Figure 27: Effect of FLAG-importin 13 overexpression on subcellular localization of GFP-GST-tagged SILAC export cargo candidates. HeLa P4 cells were transiently cotransfected with plasmids coding for GFP-GST-tagged proteins from the SILAC screen and FLAG-importin 13 or an empty control vector (pEGFP-GST) using the calcium phosphate method. FLAG-importin 13 was visualized by indirect immunofluorescence with an anti-FLAG antibody. See Table 16 for summary of importin 13 effect. The scale bars correspond to $20 \mu \mathrm{m}$. FEN1, NSUN2 and XRCC5 were tested by Christiane Spillner. 
Table 16: Effect of importin 13 overexpression on importin 13 export cargo candidates identified in SILAC screen ${ }^{\#}$

\begin{tabular}{|c|c|c|c|c|c|c|}
\hline \multicolumn{7}{|c|}{ Potential im portin 13 export cargoes } \\
\hline Uniprot ID & \multicolumn{2}{|c|}{ Proteins identified for Im p13+Ran/Im p13 } & $\frac{\text { Cargo-HA }}{\text { Ran } / \operatorname{lmp13}}$ & Cargo-HA + LMB** & GFP-GST-cargo & Summary \\
\hline \multicolumn{7}{|c|}{$\log 2(\operatorname{Imp} 13+\operatorname{Ran} / \operatorname{Imp} 13) \geq 3$} \\
\hline 000442 & RNA 3-terminal phosphate cyclase & RTCA & $\checkmark$ & & $\checkmark \checkmark$ & $\checkmark \checkmark$ \\
\hline P39748 & Flap endonuclease 1 & FEN1 & & & $\checkmark$ & $\checkmark$ \\
\hline Q6IPR3 & tRNA w ybutosine-synthesizing protein 3 homolog & TYW3 & $x$ & $x$ & $x$ & $x$ \\
\hline P27695 & DNA-(apurinic or apyrimidinic site) lyase & APEX1 & $x$ & & $\checkmark \checkmark$ & $\checkmark \checkmark$ \\
\hline P37108 & Signal recognition particle $14 \mathrm{kDa}$ protein & SRP14 & $x$ & & $x$ & $\boldsymbol{x}$ \\
\hline Q08J23 & tRNA (cytosine(34)-C(5))-methyltransferase & NSUN2 & $\checkmark \checkmark$ & & $\checkmark \checkmark$ & $\checkmark \checkmark$ \\
\hline \multicolumn{7}{|c|}{$\log 2(\operatorname{Imp} 13+R a n / \operatorname{Imp} 13) \geq 1.5$} \\
\hline HOYA96 & Heterogeneous nuclear ribonucleoprotein D0 & HNRNPD & $\checkmark \checkmark$ & & $x$ & $\checkmark \checkmark$ \\
\hline P13010 & X-ray repair cross-complementing protein 5 & $\mathrm{XRCC5}$ & $\checkmark \checkmark$ & & $\checkmark \checkmark$ & $\checkmark \checkmark$ \\
\hline Q9NTK5 & Obg-like ATPase 1 & OLA1 & $x$ & & $x$ & $x$ \\
\hline Q9Y3F4 & Serine-threonine kinase receptor-associated protein & STRAP & $\checkmark$ & & $x$ & $\checkmark$ \\
\hline Q9UK59 & Lariat debranching enzyme & DBR1 & $x$ & & $x$ & $x$ \\
\hline P25205 & DNA replication licensing factor $\mathrm{MCM} 3$ & MCM3 & $x$ & & $x$ & $x$ \\
\hline P20290 & Transcription factor BTF3 & BTF3 & $x$ & $\checkmark \checkmark$ & $x$ & $\checkmark \checkmark$ \\
\hline P41214 & Eukaryotic translation initiation factor $2 \mathrm{D}$ & EIF2D & $x$ & $\checkmark \checkmark$ & $x$ & $\checkmark \checkmark$ \\
\hline \multicolumn{7}{|c|}{$\log 2(\operatorname{Imp} 13+\operatorname{Ran} / \operatorname{Imp} 13) \geq 0.5$} \\
\hline P12956 & X-ray repair cross-complementing protein 6 & $\mathrm{XRCC6}$ & $x$ & & $\checkmark$ & $\checkmark$ \\
\hline P19525 & Interferon-induced, double-stranded RNA-activated protein kinase & EF2AK2 & $x$ & $\mathbf{x}$ & & $x$ \\
\hline 014744 & Protein arginine $\mathrm{N}$-methyltransferase 5 & PRMT5 & $x$ & $x$ & $x$ & $x$ \\
\hline Q9BQA1 & Methylosome protein 50 & WDR77 & $\mathscr{V}^{*}$ & & $x$ & $\checkmark^{*}$ \\
\hline Q01105 & Protein SET & SET & $x$ & & $\checkmark \checkmark$ & $\checkmark \checkmark$ \\
\hline \multicolumn{7}{|c|}{ Export control } \\
\hline P47813 & Eukaryotic translation initiation factor $1 \mathrm{~A}$ & EF1A & & & $\checkmark$ (GFP-tag) & $\checkmark$ \\
\hline
\end{tabular}

\#: two green ticks: strong importin 13 effect; one green tick: weak importin 13 effect; black cross: no importin13 effect

*: protein redistributed to nucleus not cytoplasm

**: cells were treated with $10 \mathrm{nM} \mathrm{LMB}$ for 2 hours, to shift the cytoplasmic protein to the nucleus 
Previously, BTF3, PRMT5, EIF2D and SQSTM1 have been reported to be Crm1 export cargo candidates $(82,32)$, therefore it was tested whether these proteins as well as TYW3 and EIF2AK2 localize to the nucleus in cells treated with the Crm1 inhibitor leptomycin B (LMB) (Figure 28). Snurportin 1 was used as a control for the specificity of the LMB effect, as it is a well-established Crm1 cargo and was not identified as an importin 13 cargo candidate. Of the six proteins tested, BTF3, EIF2D and SQSTM1 were relocalized to the nucleus in LMB treated cells. Next it was analyzed whether importin 13 can reverse the LMB effect and indeed all three proteins were redistributed to the cytoplasm in cells expressing importin 13. Including the LMB sensitive cargoes, in total 12 out of 19 export cargo candidates were affected by importin 13 coexpression.

As several importin 13 cargo candidates were identified to be LMB sensitive, the redundancy of cargo candidates for importin 13 and Crm1 was compared. A total of 1,072 Crm1 cargo candidates were identified in a study to bind to immobilized Crm1 in a RanGTP-dependent manner (32), of these 56 proteins were identified as potential importin 13 cargoes in the SILAC screen (Table S11). The identified importin 13 cargo candidates were also compared with proteins identified in a study by Kimura et al., 2017 (76) to interact with different importins. The largest overlap was found for importin 5, of 303 potential importin 5 cargoes identified, 32 were also detected in the SILAC screen as putative importin 13 cargoes (Table S12).

In summary, the SILAC based pull-down screen allows for the effective identification of potential importin 13 export cargoes. While proteins with a log2 SILAC ratio < 3 showed ambiguous results, proteins with a log2 SILAC ratio $\geq 3$ can be classified as high confidence importin 13 export cargoes. Although the screen allowed for the effective identification of known importin 13 import cargoes (3.3.3.2), only few of the tested SILAC cargo candidates could be verified as potential importin 13 import cargoes by overexpression experiments. Based on this overexpression screen, RTCA, FEN1, APEX1, NSUN2, HNRNPD, XRCC5, STRAP, BTF3, EIF2D, XRCC6 and SET were validated as potential importin 13 export cargoes, while ERI1 and NELFCD were identified as potential import cargoes. Further, PDCD4, WDR61, GTF2F2, XPA, SQSTM1, RBM22 and NELFA were identified as potential importin 13 interaction partners. 

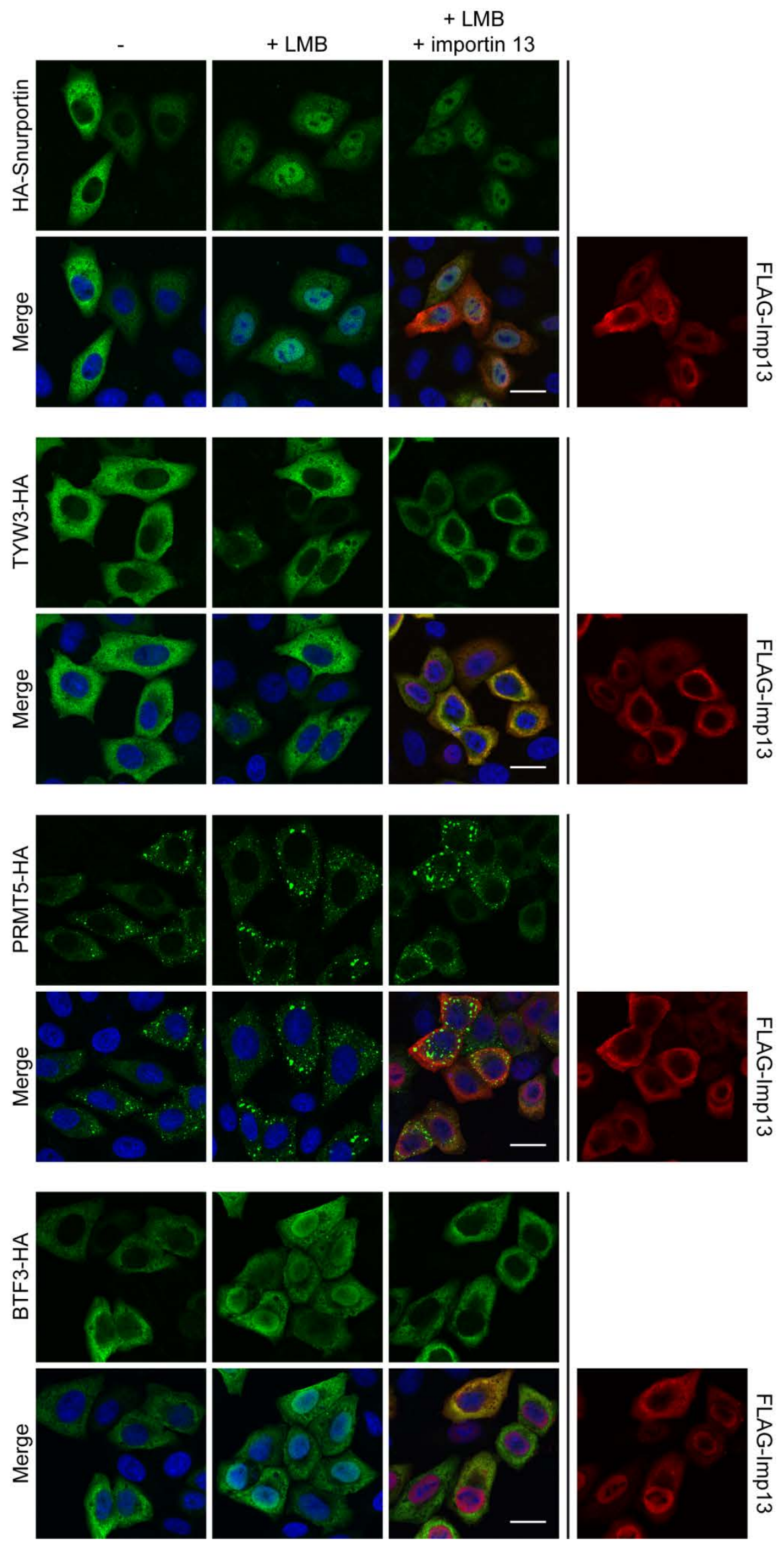

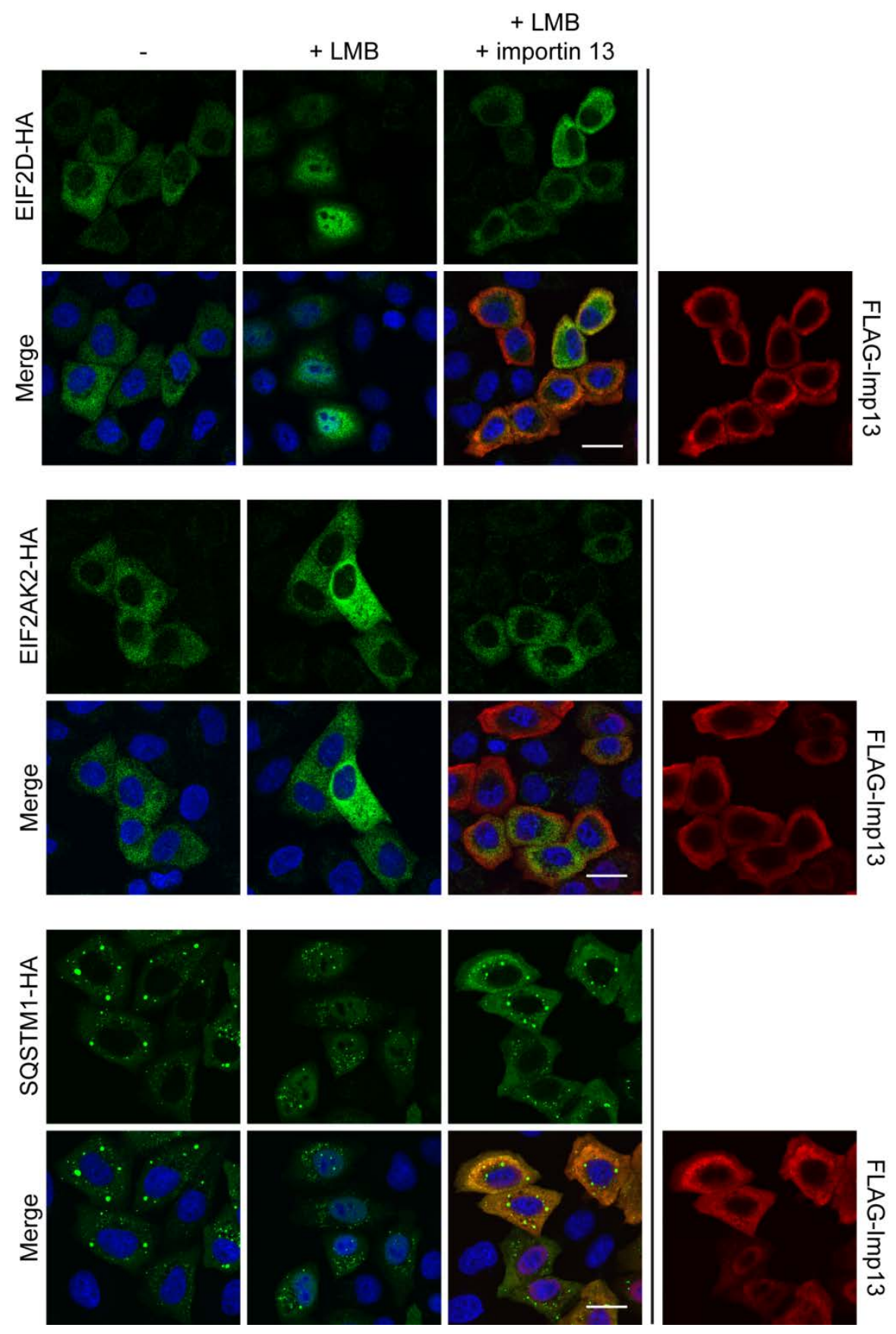

Figure 28: Effect of FLAG-importin 13 overexpression on subcellular localization of HA-tagged SILAC cargo candidates in leptomycin B treated cells. HeLa P4 cells were transiently cotransfected with plasmids coding for HA-tagged proteins from the SILAC screen and FLAG-importin 13 or an empty control vector (pcDNA3.1-HA) using the calcium phosphate method. Prior to fixation cells were treated with $10 \mathrm{nM}$ leptomycin B, a Crm1 export inhibitor, for 2 hours. FLAG-importin 13 and HA-substrates were visualized by indirect immunofluorescence with an antiFLAG and anti-HA antibody, respectively. The scale bars correspond to $20 \mu \mathrm{m}$. 


\subsection{Characterization of the Interaction of Importin 13 with Importin 13 Cargo Candidates}

Similar to the experiments performed in section 3.2.2 and 3.2.3.2, cargo candidates affected by importin 13 overexpression were characterized for their interaction with importin 13. To test whether the cargo candidates NSUN2, HNRNPD, XRCC6, FEN1, PDCD4 and ERI1 bind importin 13 through the same key residues as the known export cargo elF1A, subcellular localization of these proteins in the absence or presence of importin 13 wild type or mutant Glu436Arg/Asp481Arg, which is deficient in elF1A export, was analyzed (Figure 29). With the exception of elF1A, all proteins tested changed their localization upon coexpression of FLAG-importin 13-E436R/D481R. This demonstrates that the novel cargo candidates have a different binding site on importin 13 than elF1A or that more residues need to be mutated to abolish the interaction.

To assess as to where these newly identified importin 13 cargo candidates might bind, the effect of an N-terminal (amino acids 1-669) and a C-terminal importin 13 fragment (amino acids 526-963) on the localization of these proteins was analyzed. The C-terminal fragment is a physiologically relevant, testis-specific form of importin 13 that lacks the N-terminal RanGTP binding site and has been suggested to function as a negative regulator of nuclear import. HeLa P4 cells were transfected with plasmids coding for cargo candidates and analyzed for any changes in subcellular localization in the presence or absence of the importin 13 fragments. The known export cargo elF1A was relocalized to cytoplasm by full-length importin 13 but was not affected by the importin 13 fragments. This finding is in accordance with previous observations that elF1A mainly binds to the middle region of importin 13. In contrast, the established import cargo Ubc9, that is known to bind to the $\mathrm{N}$-terminus of importin 13 , was indeed relocalized to the nucleus by the $\mathrm{N}$ terminal fragment but not the C-terminal fragment. Similar to Ubc9, all cargo candidates tested, namely NSUN2, HNRNPD, XRCC6, FEN1 and PDCD4, with the exception of ERI1, were relocalized by both full-length importin 13 and the $\mathrm{N}$-terminal fragment but not by the C-terminal fragment. ERI1, as eIF1A, changed its subcellular localization only in the presence of full-length importin 13. Note that the expression levels of the C-terminal fragment were generally lower than that of the N-terminal importin 13 fragment.

In summary, the novel cargo candidates tested bind importin 13 differently to elF1A and the far C-terminus of importin 13 is dispensable for their interaction, with the exception of ERI1, whose subcellular localization was only affected by full-length importin 13 . 

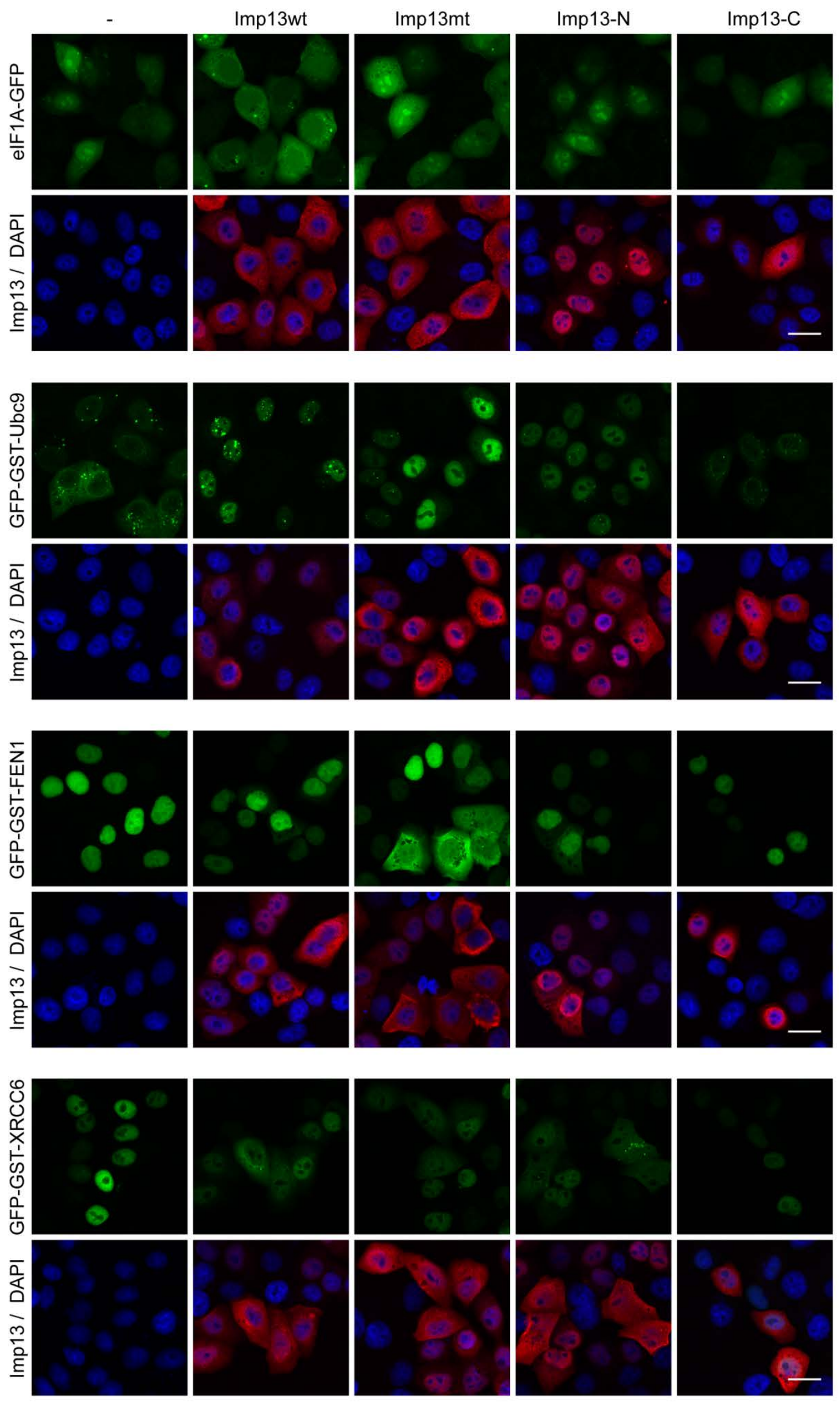

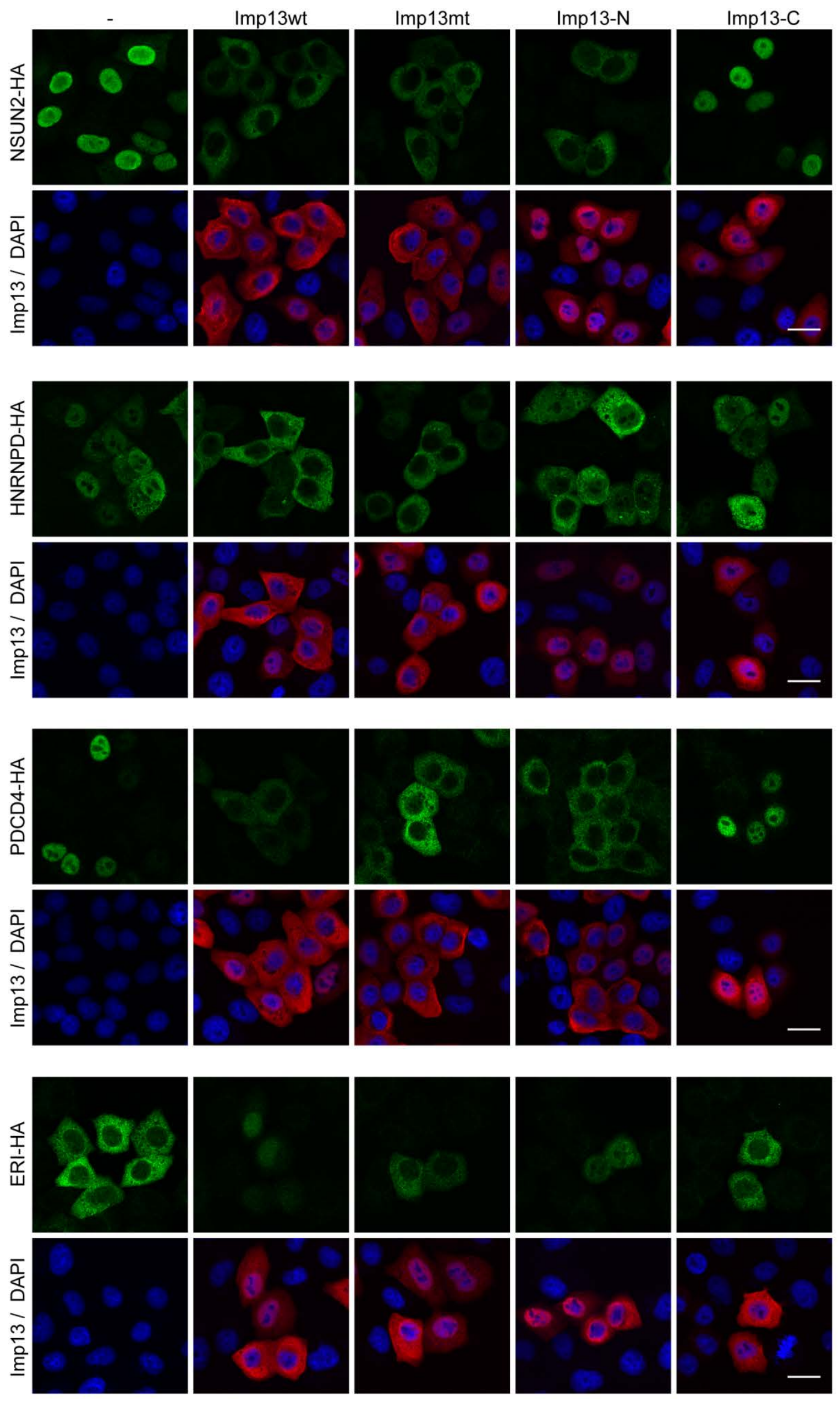
Figure 29: SILAC cargo candidates bind importin 13 differently to eIF1A and the C-terminus of importin 13 is dispensable for their interaction. HeLa P4 cells were transiently cotransfected using the calcium phosphate method with plasmids coding for HA-or GFP-GST-tagged proteins from the SILAC screen and FLAG-importin 13 wild type (Imp13wt), FLAG-importin 13E436R/D481R (Imp13mt, importin 13 mutant impaired in elF1A export); FLAG-importin 13 (aa 1669) (Imp13-N) and FLAG-importin 13 (aa 526-963) (Imp13-C) or an empty control vector. FLAGimportin 13 and HA-substrates were visualized by indirect immunofluorescence with an anti-FLAG and anti-HA antibody, respectively. The scale bars correspond to $20 \mu \mathrm{m}$.

\subsection{Functional Roles of Novel Importin 13 Cargo Candidates}

To gain insight into what cellular pathways importin 13 might regulate through transport of specific cargoes, a gene ontology enrichment analysis was done. In total 38 gene ontology clusters were identified if an enrichment score cut-off of 1.5 was applied. Table 17 shows a summary of the 10 most enriched clusters, with three representative enriched GO terms. The analysis suggests that importin 13 influences the subcellular distribution of proteins involved in processes such as translation, microtubule and cytoskeleton organization, mRNA processing and chromosome organization.

Table 17: Gene Ontology (GO) analysis of importin 13 cargo candidates ${ }^{\#}$

\begin{tabular}{|c|c|c|}
\hline GO Term & Count & p-value \\
\hline \multicolumn{3}{|c|}{ Annotation Cluster 1, Enrichment Score: 13.26} \\
\hline cellular component organization or biogenesis & 143 & $4.60 \mathrm{E}-15$ \\
\hline cellular component organization & 139 & 3.00E-14 \\
\hline organelle organization & 99 & 1.20E-12 \\
\hline \multicolumn{3}{|c|}{ Annotation Cluster 2, Enrichment Score: 11.33} \\
\hline organonitrogen compound metabolic process & 54 & 1.80E-05 \\
\hline cellular amide metabolic process & 45 & 1.90E-12 \\
\hline translation & 42 & 4.70E-17 \\
\hline \multicolumn{3}{|c|}{ Annotation Cluster 3, Enrichment Score: 11.19} \\
\hline multi-organism process & 60 & 8.50E-07 \\
\hline interspecies interaction between organisms & 49 & $6.60 \mathrm{E}-15$ \\
\hline viral process & 47 & 3.90E-14 \\
\hline \multicolumn{3}{|c|}{ Annotation Cluster 4, Enrichment Score: 9.31} \\
\hline cell adhesion & 54 & 3.70E-09 \\
\hline biological adhesion & 54 & 4.20E-09 \\
\hline cell-cell adhesion & 48 & 7.40E-12 \\
\hline \multicolumn{3}{|c|}{ Annotation Cluster 5, Enrichment Score: 7.77} \\
\hline cellular component biogenesis & 81 & $4.40 \mathrm{E}-11$ \\
\hline macromolecular complex subunit organization & 73 & 3.70E-11 \\
\hline protein complex assembly & 40 & 2.10E-05 \\
\hline \multicolumn{3}{|c|}{ Annotation Cluster 6, Enrichment Score: 6.58} \\
\hline multi-organism metabolic process & 14 & $6.80 \mathrm{E}-06$ \\
\hline formation of translation preinitiation complex & 11 & 2.70E-13 \\
\hline viral gene expression & 11 & 2.80E-04 \\
\hline
\end{tabular}




\begin{tabular}{|c|c|c|}
\hline \multicolumn{3}{|c|}{ Annotation Cluster 7, Enrichment Score: 6.36} \\
\hline cytoskeleton organization & 36 & 4.10E-06 \\
\hline microtubule-based process & 26 & 9.70E-07 \\
\hline microtubule cytoskeleton organization & 21 & 1.40E-06 \\
\hline \multicolumn{3}{|c|}{ Annotation Cluster 8, Enrichment Score: 5.19} \\
\hline cytoplasmic translational initiation & 6 & 2.30E-06 \\
\hline IRES-dependent translational initiation & 5 & 1.50E-05 \\
\hline cap-independent translational initiation & 5 & 3.90E-05 \\
\hline \multicolumn{3}{|c|}{ Annotation Cluster 9, Enrichment Score: 5.08} \\
\hline RNA processing & 34 & 1.90E-07 \\
\hline mRNA metabolic process & 31 & 7.90E-09 \\
\hline mRNA splicing, via spliceosome & 16 & 1.40E-05 \\
\hline \multicolumn{3}{|c|}{ Annotation Cluster 10, Enrichment Score: 5} \\
\hline chromosome organization & 35 & 2.20E-05 \\
\hline DNA metabolic process & 33 & 4.00E-06 \\
\hline telomere maintenance & 15 & 3.50E-09 \\
\hline
\end{tabular}

\#: importin 13 cargo candidates listed in Table S6, Table S7 and Table S8 were subjected to gene ontology analysis with the DAVID software, only three representative GO terms per cluster are shown. Count: number of genes per GO term; p-value: EASE score (modified Fisher Exact P-Value). 


\section{Discussion}

The mechanisms of nucleocytoplasmic transport are well understood. Rather little is known, however, about the biological significance of individual nuclear transport receptors. A major restriction here is the limited number of reported cargo proteins. Only recently, a large proteomics screen (76) identified multiple cargo proteins specific for the twelve species of human import receptors, including importin 13. The authors, however, did not screen for potential importin 13 export cargoes, which was a major goal of the work presented here. To expand the number of importin 13 substrates and specifically importin 13 export cargoes, three different approaches were used in this work, including an importin 13 overexpression screen using a library of nuclear proteins, an importin 13 binding experiment followed by mass spectrometry and an importin 13 binding experiment coupled to quantitative proteomics using SILAC.

\subsection{Characterization of Known Importin 13 Cargoes}

To allow for characterization of newly identified importin 13 substrates, various cellular and biochemical assays were established with the known importin 13 import cargo Ubc9 and the export cargo elF1A. Overall, the performed assays confirmed previous findings. In addition, a few observations were made that have not been previously discussed.

Endogenous eIF1A has been shown to be primarily localized in nucleoli (107), whereas in this study a homogenous distribution of endogenous eIF1A between nucleoli and the nucleoplasm was observed. As different antibodies were used, it is possible that both antibodies recognize different epitopes on eIF1A that are partially occluded if elF1A is complexed to other proteins, such as the $40 \mathrm{~S}$ ribosomal subunit. The primarily nuclear localization of elF1A is rather unique, as the vast majority of translation factors are thought to be restricted to the cytoplasm to prevent nuclear translation or interference with ribosome biogenesis by untimely interactions with pre-ribosomes (189). In turn, elF1A has been suggested to have a yet unidentified nuclear function and that importin 13 mediated export is required to ensure efficient translation by maintaining sufficient cytoplasmic levels of elF1A (189).

The primarily nuclear localization of endogenous elF1A suggests that importin 13 mediated export of elF1A is rate limiting in HeLa P4 cells. Indeed, overexpression of importin 13 resulted in a more cytoplasmic localization of endogenous elF1A, confirming that importin 13 is rate limiting for its export. In contrast, overexpressed HA-Ubc9 already localized to the nucleus without importin 13 coexpression. This suggests that either 
importin 13 is not rate limiting for Ubc9 nuclear import or Ubc9, as elF1A, is small enough $(18 \mathrm{kDa})$ to diffuse into the nucleus, where it gets sequestrated through interaction with nuclear components. Likely, both diffusion and importin 13 mediated transport play a role in Ubc9 nuclear localization, as GFP-GST-Ubc9, which should be above the diffusion limit of the NPC, requires importin 13 coexpression not only to enter the nucleus but also to maintain an exclusively nuclear localization. This shows that in contrast to HA-Ubc9, endogenous importin 13 is rate limiting for the nuclear localization of GFP-GST-Ubc9. Thus, an additional mechanism such as diffusion into the nucleus is essential to confine Ubc9 to the nucleus. Another indicator that importin 13 helps to confine Ubc9 to the nucleus is the result of proximity ligation assays, where the majority of importin 13/Ubc9 in situ interactions were detected in the nucleus. Less transient interactions in the nucleus might prevent immediate redistribution to the cytoplasm by diffusion. In this regard, an effect of importin 13 knock-down on the subcellular localization of Ubc9 should be analyzed, to determine if importin 13 is essential for the nuclear localization of Ubc9.

The notion that importin 13 is rate limiting for at least some of its cargoes in HeLa P4 cells is further supported by the observation that endogenous importin 13 levels in HeLa P4 cells as well as other cancer cell lines is very low with levels around 100-350 nM. In comparison, other nuclear transport receptors have a several fold higher cellular concentration of 1-4 $\mu \mathrm{M}(77,185)$. It has previously been demonstrated that the transport efficiency of nuclear import receptors depends on their cellular concentration $(83,192)$. Similarly, cellular Crm1 concentrations were reported to be rate limiting for export of overexpressed cargo proteins in HeLa P4 cells (193).

In summary, importin 13 is rate limiting for nucleocytoplasmic transport of its cargoes Ubc9 and eIF1A. Importin 13 mediated export of eIF1A might be essential for efficient translation by ensuring sufficient cytoplasmic levels of elF1A. Import of Ubc9 mediated by importin 13 could be required to confine Ubc9 to the nucleus and thus to prevent unspecific interactions in the cytoplasm. Indeed, many of the known Ubc9 SUMOylation targets are nuclear proteins (194) and importin 13 could function as a chaperone to prevent untimely SuMOylation.

\subsection{Overexpression Experiments for the Identification of Importin 13 Cargoes}

\subsubsection{Are DBC-1, DMAP1, TERT, DDX43 and DDX59 Importin 13 Cargoes?}

In an overexpression screen for potential importin 13 export cargoes, several nuclear proteins that were affected by importin 13 overexpression and showed an increased 
cytoplasmic localization were identified. The observed effects appear to be specific, as two of the identified proteins, DBC-1 and c-Jun, have previously been shown to interact with importin 13 in binding experiments $(49,117)$. While the importin 13 effect was largely independent of the cell line and fusion tag used, cell to cell variation could be observed within one experiment. Possible explanations could be cell cycle specific effects or higher importin 13 expression levels in cells with strong cytoplasmic accumulation.

Several different mechanisms could account for the increased cytoplasmic localization of the identified proteins upon importin 13 coexpression, including direct or indirect stimulation of nuclear export as well as reduced or inhibited nuclear import. In this regard, a testis-specific form of importin 13 (amino acids 526-963) has been reported to function as a negative regulator of nuclear import (136), a mechanism that might also apply to fulllength importin 13. Further, several transport cargoes have been reported to interact with various nuclear transport receptors in vitro but it has been suggested that in vivo a preference for a specific nuclear transport receptor might exist (195). Thus importin 13 might only bind to the identified proteins if overexpressed, thereby competing with the preferred nuclear transport receptor. Fos and c-Jun for example are proteins that have been reported to bind to several different karyopherins in vitro $(117,196)$.

To further investigate these options, transport assays, heterokaryon assays or fluorescence recovery after photobleaching (FRAP) experiments, similar to the ones performed for the importin 13 export cargoes HMG20A and EIF4G2 (136), could be performed. Another approach could be siRNA mediated knock-down of importin 13. However, RNA interference experiments with importin 13 so far had no effect on established importin 13 cargoes (data not shown), possibly due to the observed low endogenous importin 13 levels in HeLa P4 cells. If some or all of the identified proteins are indeed importin 13 export cargoes, they bind importin 13 differently to elF1A, as the importin 13 mutant impaired in eIF1A export was still fully functional in mediating relocalization of DBC-1, DMAP1, DDX43, DDX59 and TERT. This, is not surprising, as importin 13 has been demonstrated to bind its cargoes through different binding sites and key residues $(92,93,107)$.

At least DBC-1 and c-Jun appear not to be importin 13 export cargoes, as their interaction with importin 13 is reduced in the presence of $\operatorname{RanGTP}_{\mathrm{Q} 69 \mathrm{~L}}(49,117)$. Further, DBC-1 came up as a potential importin 13 import cargo in the SILAC screen, but only in one of the replicate experiments. Import assays should be done to test whether importin 13 can import DBC-1 or the other identified proteins. Import assays in permeabilized cells with c-Jun have previously been performed, but no efficient import by importin 13 could be 
detected (117). Still, silencing of importin 13 in pterygium epithelial cells has been reported to block the nuclear translocation of c-Jun (142). None of the other proteins identified in the overexpression screen were detected in the SILAC screen. This could be due to the cell extraction method using digitonin, which is known to selectively permeabilize the cell membrane but to leave the nuclear envelope largely intact $(179,180)$. Consequently, larger proteins such as TERT $(127 \mathrm{kDa})$ as well as proteins tethered to nuclear structures or larger protein complexes might not be efficiently released from the nucleus with this extraction method. Further, the endogenous concentrations of these proteins in HeLa P4 cells might be too low, preventing their identification.

In summary, the overexpression screen seems to allow for the identification of importin 13 interaction partners but not for the specific identification of importin 13 export cargoes. Importin 13 could regulate the subcellular localization of the identified proteins either through facilitated transport or through negative regulation of nuclear transport. In the case of DBC-1, importin 13 seems to function rather as a negative regulator of nuclear import than as an exportin (see section 4.3.2.2 for alternative explanations). The advantage of the overexpression screen is that it requires little optimization and provides direct information on the subcellular localization of the proteins analyzed, but it is limited by the number of available plasmids.

\subsubsection{Interaction of Importin 13 and DBC-1}

One of the cargoes analyzed in more detail for its interaction with importin 13 was DBC-1. DBC-1 regulates several nuclear proteins, such as nuclear receptors (androgen receptor, estrogen receptor $\alpha$ and $\beta$, retinoic acid receptor, thyroid hormone receptor, heam receptor Rev-erba), deacetylases (SIRT1, HDAC3) and the methyltransferase SUV39H1 (197). Previously, the coiled-coil domain of DBC-1 has been suggested to be involved in the translocation of DBC-1 through the NPC (198). Indeed, the coiled-coil domain of DBC-1 fused to a cNLS was relocalized to the cytoplasm upon importin 13 coexpression. To gain insights into which region of importin 13 is required for its interaction with DBC-1, different importin 13 fragments were coexpressed with DBC-1. Only N-terminal but not C-terminal importin 13 fragments promoted a cytoplasmic localization of DBC-1 similar to full-length importin 13. Nevertheless, as discussed above, this could be due to different mechanisms, like active export or negative regulation of import. Although, one might expect that importin 13 deletion constructs are impaired in shuttling through the NPC, an importin 13 
$\mathrm{N}$-terminal fragment has been shown to still be capable of shuttling, albeit at a slower rate than full-length importin 13 (162).

In conclusion, importin 13 interacts with the coiled-coil domain of DBC-1 and at least one binding site on the $\mathrm{N}$-terminus of importin 13 is required for their interaction.

\subsubsection{Functional Link Between Importin 13 and Nup358 Mediated Transport?}

Interestingly, the proteins that were affected the strongest by importin 13 overexpression, DBC-1, DMAP1, DDX43 and DDX59, were also identified in a screen for Nup358-dependent proteins (49). In this study, Nup358 was shown to be required not only for the nuclear localization of these proteins but to also bind DBC-1 and DMAP1 directly. Consequently, Nup358 was proposed to function as an assembly platform for import complexes (49). The strong overlap of proteins affected by Nup358 depletion and importin 13 overexpression suggests a functional link between importin 13 and Nup358, with the latter possibly serving as an assembly or disassembly platform for importin 13 transport complexes. However, the observed overlap could also be due to a general involvement of Nup358 in nucleocytoplasmic transport, as Nup358 has been implicated in various transport pathways, including importin $\alpha / \beta$, transportin 1 , transportin 3 , CAS, importin 7 and Crm1 (25). Previous binding experiments between importin 13 and a C-terminal Nup358 fragment present in a complex with Ubc9 and SUMOylated RanGAP showed no interaction (25). However, other regions of Nup358 might be involved. Binding assays should be done with importin 13 and the identified cargo candidates in the absence or presence of Nup358 wild type or deletion constructs. Importin 13 transport assays in Nup358 depleted and control cells could yield further information on a possible link.

The strong overlap of cargoes showing a dependency on both Nup358 and importin 13 suggests a functional link between the two proteins. Further experiments will be required to assess the nature of this overlap. 


\subsection{Mass Spectrometry Based Identification of Importin 13 Cargoes}

\subsubsection{Mass Spectrometry Based Methods for the Identification of $\beta$-karyopherin Cargoes}

Different approaches have been used to identify nuclear transport receptor interaction partners, including the yeast-two hybrid system $(136,199)$ and mass spectrometry approaches $(32,135,189,200)$. Further, stable isotope labeling with amino acids in cell culture (SILAC), followed by quantitative mass spectrometry has been applied to identify importin $\alpha / \beta$, transportin and Crm1 cargoes (76,80-82, 201). Compared to the previous SILAC methods, the SILAC approach applied here is limited in the identification of transient interactions, but has the major advantage of specifically identifying both nuclear import and export cargoes. Of the importin 13 cargo candidates detected in this study, 11\% were also identified in the SILAC-Tp study by Kimura et al., 2017 (76), showing that

both approaches might effectively complement each other (Table S10). One of the major cargo candidates identified in the SILAC-Tp study, but not in this study, was the integral membrane protein LRRC59 (202). LCCR59 as well as other integral membrane proteins were not detected in the approach presented here, likely as integral membrane proteins are not extracted with digitonin. It remains unclear why an integral membrane protein should be a substrate for importin 13.

\subsubsection{SILAC Based Importin 13 Binding Assays for the Identification of Importin 13 Import and Export Cargoes}

The majority of the known importin 13 import and export cargoes were identified with the SILAC based pull-down assay, proving the utility of this approach in identifying novel importin 13 cargoes. Not all of the reported importin 13 substrates (Table 1) were identified in the SILAC screen. However, some of them, for instance the glucocorticoid receptor, CCCTC-binding factor (CTCF), and myopodin, have not been validated as cargoes in transport assays. Thus, interaction partners such as myopodin might not be transported by importin 13 and instead be involved in other cellular functions of importin 13. None of the paired homeodomain transcription factors that have been demonstrated to be imported by importin 13 in transport assays were identified in our SILAC screen. Perhaps they are tethered to other nuclear components such as proteins or chromatin, preventing their extraction with digitonin from HeLa P4 cells, a possibility that could also apply to other proteins. Many of the reported importin 13 cargoes were identified in other cell lines than HeLa P4 or were found to be developmentally regulated. Thus, HeLa P4 cells might not be 
the ideal source to show an interaction of these proteins with importin 13. Since the endogenous levels of these proteins could be rather low in HeLa P4 cells, they possibly would not be detected by mass spectrometry. Certain substrates might also yield few or no ionizable tryptic peptides.

According to the SILAC screen, many importin 13 binding proteins were regulated neither by Ubc9 nor RanGTP ${ }_{\mathrm{Q} 69 \mathrm{~L}}$ in their binding to immobilized importin 13. These could be unspecific binding proteins that were not sufficiently removed by preincubation with Hzz-IgG-Sepharose or proteins that bind to other regions on importin 13 than Ubc9 and RanGTP. In total, 242 proteins were identified with the selected filtering criteria, whose binding to importin 13 was regulated by Ubc9 or RanGTP ${ }_{\mathrm{Q} 69 \mathrm{~L}}, 131$ of which were identified as potential import, 95 as export and 16 as ambiguous cargo candidates. The ambiguous proteins are considered to be export cargoes, as they were not only enriched in importin 13 binding reactions compared to binding reactions with excess Ubc9 (Imp13/Imp13+Ubc9), but also in importin 13 binding reactions with RanGTP ${ }_{\mathrm{Q} 69 \mathrm{~L}}$

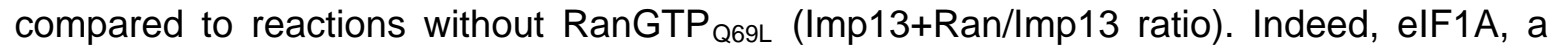
well-established importin 13 export cargo, was part of this group.

Several obligatory cytoplasmic proteins were identified as importin 13 cargo candidates. Mixing of nuclear and cytoplasmic content has been hypothesized to occur due to an imperfect NPC permeability barrier, leakage during NPC assembly, mechanical ruptures of the nuclear envelope and during open mitosis (32). Thus, importin 13 might be required to actively sort these proteins back to the cytoplasm.

\subsubsection{Novel Importin 13 Export Cargoes}

One of the first importin 13 cargoes to be identified was elF1A (135) and thus it is probably one of the major importin 13 cargoes. It was therefore expected to be detected as a high scoring protein in the SILAC screen. Indeed, it was enriched 9-fold in the importin 13 pulldown reaction with RanGTP compared to the reaction without RanGTP (Imp13+Ran/Imp13) and even 28-fold compared to the reaction with Ubc9 (Imp13+Ran/Imp13+Ubc9). The higher enrichment for the Imp13+Ran/Imp13+Ubc9 ratio compared to the Imp13+Ran/Imp13 ratio illustrates that Ubc9 is an effective tool to prevent the formation of export complexes. Further, this shows that elF1A can bind to importin 13 in the absence of RanGTP, as has previously been reported (135). Only export cargoes that bind to importin 13 in a non-cooperative manner, like elF1A, are expected to be identified in the pull-down reaction with importin 13 alone. In contrast, export cargoes that 
require cooperative binding with RanGTP are not expected to be identified in this reaction. This is based on the assumption that RanGTP is only present in its GDP-bound form in the cell extract due to the activity of cytoplasmic RanGAP. The other reported importin 13 export cargo HMG20A was not detected in the SILAC screen and EIF4G2 was identified as a low confidence export cargo. As these proteins were originally identified as interactors of the testis-specific form of importin 13 in a yeast-two hybrid screen using a human testis cDNA library, these proteins might be testis-specific importin 13 cargoes.

In total 12 out of 19 export cargo candidates tested in importin 13 overexpression experiments were relocalized to the cytoplasm in the presence of importin 13, validating them as novel export cargoes. While importin 13 overexpression did not affect all proteins tested, it does not necessarily mean that they are false positives. As demonstrated with the LMB treatment to inhibit Crm1 activity, other nuclear transport receptors can be involved in the subcellular localization of these proteins. While BTF3, EIF2D and SQSTM1 could be confirmed to be regulated by both Crm1 and importin 13 in their subcellular localization, TYW3, EIF2AK2 and PRMT5 remained cytoplasmic also after LMB treatment. These proteins might be exported by yet another exportin. Importin 13 mediated export could also be required exclusively after mitosis to sort the proteins back to their designated compartment. Knock-down of importin 13 could be a way to assess this latter possibility. Further, export cargo candidates with a nuclear localization that are not affected by importin 13 coexpression could also be part of a larger complex or require additional cofactors or post-translational modifications that are limiting in the overexpression conditions. Indeed, nuclear transport receptors have been shown to be functionally regulated by protein modifications, inhibitory factors and specific anchorings (reviewed in (88)). Thus, the effect of importin 13 overexpression or knock-down on the endogenous proteins should be analyzed. Furthermore, the observed interactions might have a regulatory function on importin 13 or be required for other cellular functions of importin 13. In this regard, nuclear transport receptors have been shown to be involved in cellular processes independent of transport such as mitosis and nuclear envelope assembly (71, 83-87).

\subsubsection{Novel Importin 13 Import Cargoes}

Apart from the known export cargo elF1A, several of the well-established importin 13 import cargoes were identified in the SILAC screen, showing that this approach can effectively distinguish between import and export cargoes. All proteins reported to be imported as heterodimers by importin 13 showed similar enrichments for both subunits in 
the SILAC experiment, further confirming that these proteins bind as heterodimers to importin 13. While the NFYB/NFYC heterodimer was enriched 1.4-fold for the binding reaction with importin 13 alone compared to binding reactions in the presence of excess Ubc9 (Imp13/Imp13+Ubc9), the CHRAC15/CHRAC17 heterodimer was enriched more than 30-fold. All of the known import cargoes were specifically enriched for the Imp13/Imp13+Ubc9 ratio, but not for the Imp13/Imp13+Ran ratio. This was surprising, as previous importin 13 pull-down experiments demonstrated that Ubc9 and Mago/Y14 can

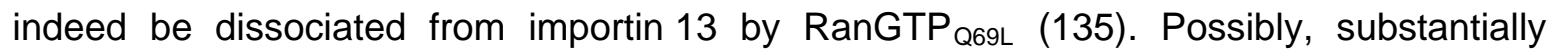
different amounts of cell lysate were used for the binding experiments and/or the loading of Ran $_{\mathrm{Q} 69 \mathrm{~L}}$ with GTP was not 100\% efficient. Consequently, limited amounts of Ran in its GTP-bound form might not be sufficient to prevent the binding of import cargoes to importin 13. However, as export cargoes were enriched in the binding reaction with RanGTP $_{\mathrm{Q} 69 \mathrm{~L}}$ (Imp13+Ran/Imp13), loading of $\mathrm{Ran}_{\mathrm{Q} 69 \mathrm{~L}}$ with GTP must have occurred to some extent.

Independent of the findings described above, proteins were selected that were enriched for either the Imp13/Imp13+Ubc9 ratio or the Imp13/Imp13+Ran ratio or both, to analyze whether import cargoes can also be identified using the Imp13/Imp13+Ran criterion. For the import cargo candidates tested, 9 out of 18 proteins were affected by importin 13 overexpression. As discussed for the export cargo candidates (4.3.2.1), proteins that could not be verified in overexpression experiments are not necessarily false positives. Additional factors or modifications that are limiting in the overexpression conditions could be required. In this regard, several known importin 13 cargoes have been shown to be imported only as heterodimers but not as individual subunits $(135,141,146,147)$. This could also apply to the subunits of the negative elongation factor (NELF) complex. While NELFA and NELFB were not affected by importin 13 overexpression, NELFCD did show a minor increase in nuclear localization. Thus, it is possible that not the individual subunits, but rather the whole NELF complex is imported by importin 13. Apart from NELFCD, other subunits of the NELF complex might also bind to importin 13, resulting in a stronger import than that of NELFCD alone. Coexpression experiments need be done for reported oligomeric proteins to test whether nuclear import is enhanced if more than one subunit is coexpressed with importin 13. In addition, binding experiments should be performed to test if binding of only the oligomer to importin 13 can be detected or also binding of the individual subunits.

PDCD4, RBM22 and NELFA fused to both an HA- and a GFP-GST-tag already showed a nuclear localization in the absence of importin 13, preventing an assessment of 
importin 13 involvement in the nuclear localization of these proteins using overexpression assays. Similar to the experiments performed with LMB to inhibit Crm1-dependent export, one could test if import of these proteins is mediated by importin $\alpha / \beta$ or transportin using the inhibitors Bimax (203) and M9M (99), respectively. Proteins relocalized to the cytoplasm could then be analyzed for importin 13 effects. The import cargo candidates ERI1 and NELFCD could be shown to relocalize to the nucleus in the presence of importin 13 overexpression. In contrast, the other seven import cargo candidates that were affected by importin 13 overexpression were not relocalized to the nucleus, but against expectations to the cytoplasm. Two of these proteins, namely GTF2F2 and PPP1CA, could indeed be export cargoes, as they showed a minor enrichment for Imp13+Ran/Imp13 of 1.3- and 1.1-fold, respectively. All other proteins were specifically enriched for importin 13 and reduced in the presence of excess RanGTP $\mathrm{Q}_{\mathrm{Q} 9 \mathrm{~L}}$ or Ubc9, emphasizing them as potential import cargoes. Several reasons could account for this observation. The interaction could be transport independent, with coexpressed importin 13 trapping the protein in the cytoplasm and preventing its nuclear import through other transport receptors. Similarly, importin 13 could function as a negative regulator of import, as it has been reported for the testis-specific form of importin $13(136,172)$. Further, importin 13 might bind to the protein, but transport could depend on a heterodimer of transport receptors as has been shown for the $\mathrm{H} 1$ histone linker that is imported by the importin $\beta$ /importin 7 heterodimer but not by the individual transport receptors $(113,114)$. The identification of importin 5 as a putative importin 13 import cargo in this work and the SILAC-Tp study (76) and the relatively high overlap of identified cargo candidates for importin 13 and importin 5 suggest that they might form a heterodimer. Finally, a protein could be both imported and exported by importin 13, depending on its interaction with other proteins or its post-translational modifications. If the export rate for one protein complex is faster than the import rate for the other complex, then the protein is expected to accumulate in the cytoplasm.

In conclusion, the SILAC screen is a more suitable approach than the importin 13 overexpression screen, allowing for the large-scale identification of both importin 13 import and export cargo candidates. Further analysis is required to show that the interaction is direct and that importin 13 mediates their nuclear transport. For this, binding assays with recombinant proteins or transport assays in permeabilized cells could be used. 


\subsubsection{Importin 13 a Bidirectional Nuclear Transport Receptor of Many Import and Export Cargoes}

When this study was started, it was unclear whether the primary function of importin 13 is nuclear import or whether it also plays a significant role in nuclear export, as elF1A was the only established importin 13 export cargo. The identification of many novel importin 13 cargo candidates in this study shows that importin 13 is indeed a bidirectional transport receptor. While bidirectional transport would be assumed to be more effective than unidirectional transport, as it can mediate transport of two cargoes with the hydrolysis of one RanGTP molecule, it does give rise to the question as to why this is not a general function of all nuclear transport receptors. As discussed by Mingot et al., 2001 (135), bidirectional transport could be limiting in the accumulation of cargoes against a chemical gradient due to the lesser expenditure of energy.

\subsubsection{Importin 13 a Negative Regulator of Nucleocytoplasmic Transport?}

Although the results obtained here demonstrate that importin 13 interacts with a large number of cellular proteins, they do not allow for differentiation of facilitated transport or inhibition of transport for all cargoes. This especially applies to the proteins identified as putative import cargoes that were relocalized to the cytoplasm and not to the nucleus upon coexpression of both full-length and an N-terminally truncated importin 13 . The $\mathrm{N}$-terminal fragment of importin 13 is expected to be functional, as FRAP experiments by Tao et al., 2004 (162) demonstrated that an N-terminal importin 13 fragment (amino acids 1-488) is still capable of shuttling, albeit at a reduced rate. Similarly, the N-terminus of importin $\beta$ has been suggested to be sufficient for mediating the nuclear import of the parathyroid hormone-related protein (PTHrP) in a Ran-dependent manner (90). FRAP and FLIP experiments as well as transport assays could be performed to test if the cargo candidates are shuttled in the presence of importin 13. As discussed earlier, additional factors or modifications might be required to facilitate importin 13 mediated import of these proteins. In this regard, it has been suggested that import cargo release depends not only on RanGTP binding, but also requires the presence of an importin 13 export cargo. Thus, overexpression experiments in the presence of an export cargo could be performed to assess if they are required for import complex disassembly. 


\subsubsection{Importin 13 an Exportin for M9 Containing Proteins?}

A curious finding of the overexpression screen was that importin 13 affected the subcellular distribution of the artificial import cargo dGFP-GST-M9. M9 is an import signal (PY-NLS) found in the heterogeneous nuclear ribonucleoproteins hnRNPA1 and hnRNPA2 that is recognized by transportin (204). hnRNPA1 functions in regulation of transcription, alternative splicing and mRNA nuclear export (205-207). The M9 sequence has been suggested to not only function as an import signal, but also as a signal sequence for nuclear export (208). Thus, importin 13 might function as an exportin for M9 containing substrates. This hypothesis is further supported by findings from the SILAC screen, where hnRNPA1 was identified as a high confidence export cargo candidate with a more than 9-fold enrichment for binding reactions in the presence of RanGTP ${ }_{\mathrm{Q} 69 \mathrm{~L}}$ compared to binding reactions without RanGTP $_{\mathrm{Q} 69 \mathrm{~L}}$ (Imp13+Ran/Imp13) (Table 18). Apart from hnRNPA1, several other heterogeneous nuclear ribonucleoproteins were identified as potential importin 13 export cargoes, namely hnRNPH1, A2B1, $A B$ and $C$ in at least one of the SILAC replicates and HNRNPD, $\mathrm{K}$, and $\mathrm{U}$ in all three biological replicates (Table 18). Of these, HNRNPD was analyzed in more detail and indeed it was relocalized to the cytoplasm in importin 13 overexpression experiments. For the validation of importin 13 as a potential exportin for M9 containing cargoes, overexpression experiments, binding assays, transport assays and mutational studies should be performed.

Table 18: Heterogeneous nuclear ribonucleoproteins (hnRNPs) identified in SILAC screen (see section 3.3.3 for experimental details)

\begin{tabular}{|c|c|c|c|c|}
\hline $\begin{array}{l}\text { Uniprot } \\
\text { ID }\end{array}$ & Protein Name & Gene & $\begin{array}{l}\text { Iog2(Imp13+Ranl } \\
\text { Imp13) }\end{array}$ & $\begin{array}{l}\text { log2(Imp13+Ran/ } \\
\text { Imp13+Ubc9) }\end{array}$ \\
\hline \multicolumn{5}{|c|}{ detected in 3 SILAC replicates } \\
\hline F8VZ49 & Heterogeneous nuclear ribonucleoprotein A1 & HNRNPA1 & 3.33691 & 3.12131 \\
\hline HOYA96 & Heterogeneous nuclear ribonucleoprotein D0 & HNRNPD & 2.96732 & 2.53505 \\
\hline P61978 & Heterogeneous nuclear ribonucleoprotein $\mathrm{K}$ & HNRNPK & 1.19892 & 2.05797 \\
\hline Q00839 & Heterogeneous nuclear ribonucleoprotein $U$ & HNRNPU & 0.939557 & 0.290223 \\
\hline \multicolumn{5}{|c|}{ detected in 2 SILAC replicates } \\
\hline E9PCY7 & Heterogeneous nuclear ribonucleoprotein $\mathrm{H}$ & HNRNPH1 & 0.430966 & 1.55829 \\
\hline \multicolumn{5}{|c|}{ detected in 1 SILAC replicates } \\
\hline P22626 & Heterogeneous nuclear ribonucleoproteins A2/B1 & HNRNPA2B1 & 1.4814 & 1.94375 \\
\hline Q99729 & Heterogeneous nuclear ribonucleoprotein A/B & HNRNPAB & 1.32688 & 0.662844 \\
\hline G3V5X6 & Heterogeneous nuclear ribonucleoproteins C1/C2 & HNRNPC & 0.542857 & -0.026021 \\
\hline
\end{tabular}




\section{Outlook}

Further validation of the identified importin 13 cargo candidates is required to confirm them as importin 13 transport cargoes. In a first step, binding experiments with recombinant proteins should be performed to show that the interaction between the identified proteins and importin 13 is direct. In a second step, these proteins should then be tested in transport assays in permeabilized cells to prove that they are indeed imported or exported in an importin 13-dependent manner. Confirmed importin 13 cargoes should then be analyzed for a possible common targeting signal that is recognized by importin 13 . Furthermore, the binding regions on importin 13 for these proteins should be identified. In addition, it could be interesting to test if importin 13 can bind the novel export cargoes in a cooperative or non-cooperative manner with RanGTP, as has been suggested for elF1A $(107,135)$.

As importin 13 is expressed only at low levels in HeLa P4 cells, the effect of importin 13 on the identified cargoes should also be analyzed in cells with higher endogenous importin 13 concentrations, such as cells derived from brain or from testis. Knock-down of importin 13 in such cells could help to verify additional importin 13 cargo candidates. Similarly, the cargo candidates could be analyzed in embryonic stem cells to determine if they are developmentally regulated.

The possible functional link between importin 13 and Nup358-dependent transport should be tested for the identified SILAC cargo candidates by knocking-down Nup358 in HeLa P4 cells or expressing inhibitory soluble Nup358 fragments (49). Complementary to this, a direct interaction between importin 13 and Nup358, Nup50 (135) or other nucleoporins should be analyzed, to gain insights into importin 13 translocation through the NPC. To this end binding assays, proximity ligation assays, RanGAP assays or siRNA mediated knock-down of specific nucleoporins could be performed.

Furthermore, the possible role of importin 13 in the export of M9 signal sequence containing proteins as well as the formation of a possible importin 13/importin 5 heterodimer should be tested using overexpression experiments, binding assays and transport assays.

Finally, it will be of interest to see how importin 13 mediated nucleocytoplasmic transport of specific cargoes affects certain cellular pathways and its possible involvement in diseases such as asthma and cancer.

Taken together, the larger spectrum of importin 13 cargoes will give new insights into importin 13 mediated nucleocytoplasmic transport. 


\section{References}

1. Alberts, B. (2008) Molecular Biology of the Cell, 5th Ed., Garland Science

2. Watson ML. (1959) Further observations on the nuclear envelope of the animal cell. The Journal of Biophysical and Biochemical Cytology 6, 147-156

3. Hoelz, A., Debler, E. W., and Blobel, G. (2011) The structure of the nuclear pore complex. Annual Review of Biochemistry 80, 613-643

4. Lin, D. H., Stuwe, T., Schilbach, S., Rundlet, E. J., Perriches, T., Mobbs, G., Fan, Y., Thierbach, K., Huber, F. M., Collins, L. N., Davenport, A. M., Jeon, Y. E., and Hoelz, A. (2016) Architecture of the symmetric core of the nuclear pore. Science 352, aaf1015

5. Kosinski, J., Mosalaganti, S., Appen, A. von, Teimer, R., DiGuilio, A. L., Wan, W., Bui, K. H., Hagen, W. J. H., Briggs, J. A. G., Glavy, J. S., Hurt, E., and Beck, M. (2016) Molecular architecture of the inner ring scaffold of the human nuclear pore complex. Science 352, 363-365

6. Appen, A. von, Kosinski, J., Sparks, L., Ori, A., DiGuilio, A. L., Vollmer, B., Mackmull, M.-T., Banterle, N., Parca, L., Kastritis, P., Buczak, K., Mosalaganti, S., Hagen, W., Andres-Pons, A., Lemke, E. A., Bork, P., Antonin, W., Glavy, J. S., Bui, K. H., and Beck, M. (2015) In situ structural analysis of the human nuclear pore complex. Nature 526, 140-143

7. Bui, K. H., Appen, A. von, DiGuilio, A. L., Ori, A., Sparks, L., Mackmull, M.-T., Bock, T., Hagen, W., Andres-Pons, A., Glavy, J. S., and Beck, M. (2013) Integrated structural analysis of the human nuclear pore complex scaffold. Cell 155, 1233-1243

8. Beck, M., Forster, F., Ecke, M., Plitzko, J. M., Melchior, F., Gerisch, G., Baumeister, W., and Medalia, O. (2004) Nuclear pore complex structure and dynamics revealed by cryoelectron tomography. Science 306, 1387-1390

9. Hinshaw, J. E., Carragher, B. O., and Milligan, R. A. (1992) Architecture and design of the nuclear pore complex. Cell 69, 1133-1141

10. Maimon, T., Elad, N., Dahan, I., and Medalia, O. (2012) The human nuclear pore complex as revealed by cryo-electron tomography. Structure 20, 998-1006

11. Ungricht, R., Klann, M., Horvath, P., and Kutay, U. (2015) Diffusion and retention are major determinants of protein targeting to the inner nuclear membrane. The Journal of Cell Biology 209, 687-703

12. Schwartz, T. U. (2016) The Structure Inventory of the Nuclear Pore Complex. Journal of Molecular Biology 428, 1986-2000 
13. Dickmanns, A., Kehlenbach, R. H., and Fahrenkrog, B. (2015) Nuclear Pore Complexes and Nucleocytoplasmic Transport: From Structure to Function to Disease. International Review of Cell and Molecular Biology 320, 171-233

14. Gall, J. G. (1967) Octagonal nuclear pores. The Journal of Cell Biology 32, 391-399

15. Reichelt, R., Holzenburg, A., Buhle, E. L., JR, Jarnik, M., Engel, A., and Aebi, U. (1990) Correlation between structure and mass distribution of the nuclear pore complex and of distinct pore complex components. The Journal of Cell Biology 110, 883-894

16. Schmidt, H. B., and Görlich, D. (2016) Transport Selectivity of Nuclear Pores, Phase Separation, and Membraneless Organelles. Trends in Biochemical Sciences 41, 46-61

17. Rout, M. P., and Blobel, G. (1993) Isolation of the yeast nuclear pore complex. The Journal of Cell Biology 123, 771-783

18. Asakawa, H., Yang, H.-J., Yamamoto, T. G., Ohtsuki, C., Chikashige, Y., SakataSogawa, K., Tokunaga, M., Iwamoto, M., Hiraoka, Y., and Haraguchi, T. (2014) Characterization of nuclear pore complex components in fission yeast Schizosaccharomyces pombe. Nucleus 5, 149-162

19. Grossman, E., Medalia, O., and Zwerger, M. (2012) Functional architecture of the nuclear pore complex. Annual Review of Biophysics 41, 557-584

20. Ribbeck, K., and Görlich, D. (2001) Kinetic analysis of translocation through nuclear pore complexes. The EMBO Journal 20, 1320-1330

21. Cronshaw, J. M., Krutchinsky, A. N., Zhang, W., Chait, B. T., and Matunis, M. J. (2002) Proteomic analysis of the mammalian nuclear pore complex. The Journal of Cell Biology 158, 915-927

22. Rout, M. P., Aitchison, J. D., Suprapto, A., Hjertaas, K., Zhao, Y., and Chait, B. T. (2000) The yeast nuclear pore complex: Composition, architecture, and transport mechanism. The Journal of Cell Biology 148, 635-651

23. Werner, A., Flotho, A., and Melchior, F. (2012) The RanBP2/RanGAP1*SUMO1/Ubc9 complex is a multisubunit SUMO E3 ligase. Molecular Cell 46, 287-298

24. Yaseen, N. R., and Blobel, G. (1999) GTP hydrolysis links initiation and termination of nuclear import on the nucleoporin nup358. The Journal of Biological Chemistry 274, 26493-26502

25. Ritterhoff, T., Das, H., Hofhaus, G., Schroder, R. R., Flotho, A., and Melchior, F. (2016) The RanBP2/RanGAP1*SUMO1/Ubc9 SUMO E3 ligase is a disassembly machine for Crm1-dependent nuclear export complexes. Nature Communications 7, 11482

26. Patel, S. S., Belmont, B. J., Sante, J. M., and Rexach, M. F. (2007) Natively unfolded nucleoporins gate protein diffusion across the nuclear pore complex. Cell 129, 83-96 
27. Ribbeck, K., and Görlich, D. (2002) The permeability barrier of nuclear pore complexes appears to operate via hydrophobic exclusion. The EMBO Journal 21, 2664-2671

28. Beck, M., and Hurt, E. (2017) The nuclear pore complex: Understanding its function through structural insight. Nature Reviews. Molecular Cell Biology 18, 73-89

29. Strambio-De-Castillia, C., Niepel, M., and Rout, M. P. (2010) The nuclear pore complex: Bridging nuclear transport and gene regulation. Nature Reviews. Molecular Cell Biology 11, 490-501

30. Hezwani, M., and Fahrenkrog, B. (2017) The Functional Versatility of The Nuclear Pore Complex Proteins. Seminars in Cell \& Developmental Biology, (Epub ahead of print)

31. Sakuma, S., and D'Angelo, M. A. (2017) The roles of the nuclear pore complex in cellular dysfunction, aging and disease. Seminars in Cell \& Developmental Biology, (Epub ahead of print)

32. Kirli, K., Karaca, S., Dehne, H. J., Samwer, M., Pan, K. T., Lenz, C., Urlaub, H., and Görlich, D. (2015) A deep proteomics perspective on CRM1-mediated nuclear export and nucleocytoplasmic partitioning. eLife 4, e11466

33. Hülsmann, B. B., Labokha, A. A., and Görlich, D. (2012) The permeability of reconstituted nuclear pores provides direct evidence for the selective phase model. Cell 150, 738-751

34. Frey, S., Richter, R. P., and Görlich, D. (2006) FG-rich repeats of nuclear pore proteins form a three-dimensional meshwork with hydrogel-like properties. Science 314, 815817

35. Frey, S., and Görlich, D. (2007) A saturated FG-repeat hydrogel can reproduce the permeability properties of nuclear pore complexes. Cell 130, 512-523

36. Rout, M. P., Aitchison, J. D., Magnasco, M. O., and Chait, B. T. (2003) Virtual gating and nuclear transport: the hole picture. Trends in Cell Biology 13, 622-628

37. Lim, R. Y. H., Fahrenkrog, B., Köser, J., Schwarz-Herion, K., Deng, J., and Aebi, U. (2007) Nanomechanical basis of selective gating by the nuclear pore complex. Science 318, 640-643

38. Kapinos, L. E., Schoch, R. L., Wagner, R. S., Schleicher, K. D., and Lim, R. Y. H. (2014) Karyopherin-centric control of nuclear pores based on molecular occupancy and kinetic analysis of multivalent binding with FG nucleoporins. Biophysical Journal 106, 1751-1762

39. Yamada, J., Phillips, J. L., Patel, S., Goldfien, G., Calestagne-Morelli, A., Huang, H., Reza, R., Acheson, J., Krishnan, V. V., Newsam, S., Gopinathan, A., Lau, E. Y., Colvin, M. E., Uversky, V. N., and Rexach, M. F. (2010) A bimodal distribution of two 
distinct categories of intrinsically disordered structures with separate functions in FG nucleoporins. Molecular \& Cellular Proteomics 9, 2205-2224

40. Melcák, I., Hoelz, A., and Blobel, G. (2007) Structure of Nup58/45 suggests flexible nuclear pore diameter by intermolecular sliding. Science 315, 1729-1732

41. Solmaz, S. R., Blobel, G., and Melcák, I. (2013) Ring cycle for dilating and constricting the nuclear pore. Proceedings of the National Academy of Sciences of the United States of America 110, 5858-5863

42. Wälde, S., and Kehlenbach, R. H. (2010) The Part and the Whole: Functions of nucleoporins in nucleocytoplasmic transport. Trends in Cell Biology 20, 461-469

43. Fried, H., and Kutay, U. (2003) Nucleocytoplasmic transport: taking an inventory. Cellular and Molecular Life Sciences 60, 1659-1688

44. Kalab, P., Weis, K., and Heald, R. (2002) Visualization of a Ran-GTP gradient in interphase and mitotic Xenopus egg extracts. Science 295, 2452-2456

45. Smith, A. E., Slepchenko, B. M., Schaff, J. C., Loew, L. M., and Macara, I. G. (2002) Systems analysis of Ran transport. Science 295, 488-491

46. Ben-Efraim, I., and Gerace, L. (2001) Gradient of increasing affinity of importin beta for nucleoporins along the pathway of nuclear import. The Journal of Cell Biology 152, 411-417

47. Hutten, S., Flotho, A., Melchior, F., and Kehlenbach, R. H. (2008) The Nup358RanGAP complex is required for efficient importin alpha/beta-dependent nuclear import. Molecular Biology of the Cell 19, 2300-2310

48. Hutten, S., Wälde, S., Spillner, C., Hauber, J., and Kehlenbach, R. H. (2009) The nuclear pore component Nup358 promotes transportin-dependent nuclear import. Journal of Cell Science 122, 1100-1110

49. Wälde, S., Thakar, K., Hutten, S., Spillner, C., Nath, A., Rothbauer, U., Wiemann, S., and Kehlenbach, R. H. (2012) The nucleoporin Nup358/RanBP2 promotes nuclear import in a cargo- and transport receptor-specific manner. Traffic 13, 218-233

50. Izaurralde, E., Kutay, U., Kobbe, C. von, Mattaj, I. W., and Görlich, D. (1997) The asymmetric distribution of the constituents of the Ran system is essential for transport into and out of the nucleus. The EMBO Journal 16, 6535-6547

51. Richards, S. A., Carey, K. L., and Macara, I. G. (1997) Requirement of guanosine triphosphate-bound ran for signal-mediated nuclear protein export. Science 276, 18421844

52. Bischoff, F. R., and Ponstingl, H. (1991) Catalysis of guanine nucleotide exchange on Ran by the mitotic regulator RCC1. Nature 354, 80-82 
53. Bischoff, F. R., Klebe, C., Kretschmer, J., Wittinghofer, A., and Ponstingl, H. (1994) RanGAP1 induces GTPase activity of nuclear Ras-related Ran. Proceedings of the National Academy of Sciences of the United States of America 91, 2587-2591

54. Rexach, M., and Blobel, G. (1995) Protein import into nuclei: Association and dissociation reactions involving transport substrate, transport factors, and nucleoporins. Cell 83, 683-692

55. Görlich, D., Panté, N., Kutay, U., Aebi, U., and Bischoff, F. R. (1996) Identification of different roles for RanGDP and RanGTP in nuclear protein import. The EMBO Journal 15, 5584-5594

56. Askjaer, P., Jensen, T. H., Nilsson, J., Englmeier, L., and Kjems, J. (1998) The specificity of the CRM1-Rev nuclear export signal interaction is mediated by RanGTP. The Journal of Biological Chemistry 273, 33414-33422

57. Monecke, T., Haselbach, D., Voss, B., Russek, A., Neumann, P., Thomson, E., Hurt, E., Zachariae, U., Stark, H., Grubmüller, H., Dickmanns, A., and Ficner, R. (2013) Structural basis for cooperativity of CRM1 export complex formation. Proceedings of the National Academy of Sciences of the United States of America 110, 960-965

58. Kehlenbach, R. H., Dickmanns, A., Kehlenbach, A., Guan, T., and Gerace, L. (1999) A role for RanBP1 in the release of CRM1 from the nuclear pore complex in a terminal step of nuclear export. The Journal of Cell Biology 145, 645-657

59. Bischoff, F. R., and Görlich, D. (1997) RanBP1 is crucial for the release of RanGTP from importin beta-related nuclear transport factors. FEBS letters 419, 249-254

60. Moore, M. S., and Blobel, G. (1994) Purification of a Ran-interacting protein that is required for protein import into the nucleus. Proceedings of the National Academy of Sciences of the United States of America 91, 10212-10216

61. Paschal, B. M., and Gerace, L. (1995) Identification of NTF2, a cytosolic factor for nuclear import that interacts with nuclear pore complex protein p62. The Journal of Cell Biology 129, 925-937

62. Ribbeck, K., Lipowsky, G., Kent, H. M., Stewart, M., and Görlich, D. (1998) NTF2 mediates nuclear import of Ran. The EMBO Journal 17, 6587-6598

63. Smith, A., Brownawell, A., and Macara, I. G. (1998) Nuclear import of Ran is mediated by the transport factor NTF2. Current Biology 8, 1403-1406

64. Bischoff, F. R., and Ponstingl, H. (1991) Mitotic regulator protein RCC1 is complexed with a nuclear ras-related polypeptide. Proceedings of the National Academy of Sciences of the United States of America 88, 10830-10834 
65. Adam, S. A., Marr, R. S., and Gerace, L. (1990) Nuclear protein import in permeabilized mammalian cells requires soluble cytoplasmic factors. The Journal of Cell Biology 111, 807-816

66. Adam, E. J., and Adam, S. A. (1994) Identification of cytosolic factors required for nuclear location sequence-mediated binding to the nuclear envelope. The Journal of Cell Biology 125, 547-555

67. Melchior, F., Paschal, B., Evans, J., and Gerace, L. (1993) Inhibition of nuclear protein import by nonhydrolyzable analogues of GTP and identification of the small GTPase Ran/TC4 as an essential transport factor. The Journal of Cell Biology 123, 1649-1659

68. Moore, M. S., and Blobel, G. (1993) The GTP-binding protein Ran/TC4 is required for protein import into the nucleus. Nature 365, 661-663

69. Görlich, D., and Kutay, U. (1999) Transport between the cell nucleus and the cytoplasm. Annual Review of Cell and Developmental Biology 15, 607-660

70. Port, S. A., Monecke, T., Dickmanns, A., Spillner, C., Hofele, R., Urlaub, H., Ficner, R., and Kehlenbach, R. H. (2015) Structural and Functional Characterization of CRM1Nup214 Interactions Reveals Multiple FG-Binding Sites Involved in Nuclear Export. Cell Reports 13, 690-702

71. Chook, Y. M., and Süel, K. E. (2011) Nuclear import by karyopherin-betas: recognition and inhibition. Biochimica et Biophysica Acta 1813, 1593-1606

72. Cook, A., Bono, F., Jinek, M., and Conti, E. (2007) Structural biology of nucleocytoplasmic transport. Annual Review of Biochemistry 76, 647-671

73. Andrade, M. A., and Bork, P. (1995) HEAT repeats in the Huntington's disease protein. Nature Genetics 11, 115-116

74. Andrade, M. A., Perez-Iratxeta, C., and Ponting, C. P. (2001) Protein repeats: structures, functions, and evolution. Journal of Structural Biology 134, 117-131

75. Pemberton, L. F., and Paschal, B. M. (2005) Mechanisms of receptor-mediated nuclear import and nuclear export. Traffic 6, 187-198

76. Kimura, M., Morinaka, Y., Imai, K., Kose, S., Horton, P., and Imamoto, N. (2017) Extensive cargo identification reveals distinct biological roles of the 12 importin pathways. eLife 6, e21184

77. Hahn, S., and Schlenstedt, G. (2011) Importin beta-type nuclear transport receptors have distinct binding affinities for Ran-GTP. Biochemical and Biophysical Research Communications 406, 383-388 
78. Kimura, M., Thakar, K., Karaca, S., Imamoto, N., and Kehlenbach, R. H. (2014) Novel approaches for the identification of nuclear transport receptor substrates. Methods in Cell Biology 122, 353-378

79. Mann, M. (2006) Functional and quantitative proteomics using SILAC. Nature Reviews. Molecular Cell Biology 7, 952-958

80. Kimura, M., Kose, S., Okumura, N., Imai, K., Furuta, M., Sakiyama, N., Tomii, K., Horton, P., Takao, T., and Imamoto, N. (2013) Identification of cargo proteins specific for the nucleocytoplasmic transport carrier transportin by combination of an in vitro transport system and stable isotope labeling by amino acids in cell culture (SILAC)based quantitative proteomics. Molecular \& Cellular Proteomics 12, 145-157

81. Kimura, M., Okumura, N., Kose, S., Takao, T., and Imamoto, N. (2013) Identification of cargo proteins specific for importin-beta with importin-alpha applying a stable isotope labeling by amino acids in cell culture (SILAC)-based in vitro transport system. The Journal of Biological Chemistry 288, 24540-24549

82. Thakar, K., Karaca, S., Port, S. A., Urlaub, H., and Kehlenbach, R. H. (2013) Identification of CRM1-dependent Nuclear Export Cargos Using Quantitative Mass Spectrometry. Molecular \& Cellular Proteomics 12, 664-678

83. Mosammaparast, N., and Pemberton, L. F. (2004) Karyopherins: from nucleartransport mediators to nuclear-function regulators. Trends in Cell Biology 14, 547-556

84. Arnaoutov, A., and Dasso, M. (2005) Ran-GTP regulates kinetochore attachment in somatic cells. Cell Cycle 4, 1161-1165

85. Budhu, A. S., and Wang, X. W. (2005) Loading and unloading: orchestrating centrosome duplication and spindle assembly by Ran/Crm1. Cell Cycle 4, 1510-1514

86. Lau, C. K., Delmar, V. A., Chan, R. C., Phung, Q., Bernis, C., Fichtman, B., Rasala, B. A., and Forbes, D. J. (2009) Transportin regulates major mitotic assembly events: from spindle to nuclear pore assembly. Molecular Biology of the Cell 20, 4043-4058

87. Dishinger, J. F., Kee, H. L., Jenkins, P. M., Fan, S., Hurd, T. W., Hammond, J. W., Truong, Y. N.-T., Margolis, B., Martens, J. R., and Verhey, K. J. (2010) Ciliary entry of the kinesin-2 motor KIF17 is regulated by importin-beta2 and RanGTP. Nature Cell Biology 12, 703-710

88. Kimura, M., and Imamoto, N. (2014) Biological significance of the importin-beta familydependent nucleocytoplasmic transport pathways. Traffic 15, 727-748

89. Imasaki, T., Shimizu, T., Hashimoto, H., Hidaka, Y., Kose, S., Imamoto, N., Yamada, M., and Sato, M. (2007) Structural basis for substrate recognition and dissociation by human transportin 1. Molecular Cell 28, 57-67 
90. Cingolani, G., Bednenko, J., Gillespie, M. T., and Gerace, L. (2002) Molecular basis for the recognition of a nonclassical nuclear localization signal by importin beta. Molecular Cell 10, 1345-1353

91. Lee, B. J., Cansizoglu, A. E., Süel, K. E., Louis, T. H., Zhang, Z., and Chook, Y. M. (2006) Rules for nuclear localization sequence recognition by karyopherin beta 2. Cell 126, 543-558

92. Bono, F., Cook, A. G., Grünwald, M., Ebert, J., and Conti, E. (2010) Nuclear import mechanism of the EJC component Mago-Y14 revealed by structural studies of importin 13. Molecular Cell 37, 211-222

93. Grünwald, M., and Bono, F. (2011) Structure of Importin13-Ubc9 complex: nuclear import and release of a key regulator of sumoylation. The EMBO Journal 30, 427-438

94. Jeong, S. A., Kim, K., Lee, J. H., Cha, J. S., Khadka, P., Cho, H.-S., and Chung, I. K. (2015) Akt-mediated phosphorylation increases the binding affinity of hTERT for importin alpha to promote nuclear translocation. Journal of Cell Science 128, 22872301

95. Niu, C., Zhang, J., Gao, F., Yang, L., Jia, M., Zhu, H., and Gong, W. (2012) FUSNLS/Transportin 1 complex structure provides insights into the nuclear targeting mechanism of FUS and the implications in ALS. PloS One 7, e47056

96. Maertens, G. N., Cook, N. J., Wang, W., Hare, S., Gupta, S. S., Öztop, I., Lee, K., Pye, V. E., Cosnefroy, O., Snijders, A. P., KewalRamani, V. N., Fassati, A., Engelman, A., and Cherepanov, P. (2014) Structural basis for nuclear import of splicing factors by human Transportin 3. Proceedings of the National Academy of Sciences of the United States of America 111, 2728-2733

97. Cingolani, G., Petosa, C., Weis, K., and Muller, C. W. (1999) Structure of importin-beta bound to the IBB domain of importin-alpha. Nature 399, 221-229

98. Lee, S. J., Sekimoto, T., Yamashita, E., Nagoshi, E., Nakagawa, A., Imamoto, N., Yoshimura, M., Sakai, H., Chong, K. T., Tsukihara, T., and Yoneda, Y. (2003) The structure of importin-beta bound to SREBP-2: nuclear import of a transcription factor. Science 302, 1571-1575

99. Cansizoglu, A. E., Lee, B. J., Zhang, Z. C., Fontoura, B. M. A., and Chook, Y. M. (2007) Structure-based design of a pathway-specific nuclear import inhibitor. Nature Structural \& Molecular Biology 14, 452-454

100. Huber, F. M., and Hoelz, A. (2017) Molecular basis for protection of ribosomal protein L4 from cellular degradation. Nature Communications 8, 14354 
101. Monecke, T., Güttler, T., Neumann, P., Dickmanns, A., Görlich, D., and Ficner, R. (2009) Crystal structure of the nuclear export receptor CRM1 in complex with Snurportin1 and RanGTP. Science 324, 1087-1091

102. Güttler, T., Madl, T., Neumann, P., Deichsel, D., Corsini, L., Monecke, T., Ficner, R., Sattler, M., and Görlich, D. (2010) NES consensus redefined by structures of PKI-type and Rev-type nuclear export signals bound to CRM1. Nature Structural \& Molecular Biology 17, 1367-1376

103. Fung, H. Y. J., Fu, S.-C., and Chook, Y. M. (2017) Nuclear export receptor CRM1 recognizes diverse conformations in nuclear export signals. eLife 6, e23961

104. Cook, A. G., and Conti, E. (2010) Nuclear export complexes in the frame. Current Opinion in Structural Biology 20, 247-252

105. Okada, C., Yamashita, E., Lee, S. J., Shibata, S., Katahira, J., Nakagawa, A., Yoneda, Y., and Tsukihara, T. (2009) A high-resolution structure of the pre-microRNA nuclear export machinery. Science 326, 1275-1279

106. Aksu, M., Trakhanov, S., and Görlich, D. (2016) Structure of the exportin Xpo4 in complex with RanGTP and the hypusine-containing translation factor elF5A. Nature Communications 7, 11952

107. Grünwald, M., Lazzaretti, D., and Bono, F. (2013) Structural basis for the nuclear export activity of Importin13. The EMBO Journal 32, 899-913

108. Matsuura, Y., and Stewart, M. (2004) Structural basis for the assembly of a nuclear export complex. Nature 432, 872-877

109. Görlich, D., Prehn, S., Laskey, R. A., and Hartmann, E. (1994) Isolation of a protein that is essential for the first step of nuclear protein import. Cell 79, 767-778

110. Goldfarb, D. S., Corbett, A. H., Mason, D. A., Harreman, M. T., and Adam, S. A. (2004) Importin alpha: a multipurpose nuclear-transport receptor. Trends in Cell Biology 14, 505-514

111. Huber, J., Cronshagen, U., Kadokura, M., Marshallsay, C., Wada, T., Sekine, M., and Lührmann, R. (1998) Snurportin1, an m3G-cap-specific nuclear import receptor with a novel domain structure. The EMBO Journal 17, 4114-4126

112. Ström, A. C., and Weis, K. (2001) Importin-beta-like nuclear transport receptors. Genome Biology 2, 3008

113. Görlich, D., Dabrowski, M., Bischoff, F. R., Kutay, U., Bork, P., Hartmann, E., Prehn, S., and Izaurralde, E. (1997) A novel class of RanGTP binding proteins. The Journal of Cell Biology 138, 65-80 
114. Jäkel, S., Albig, W., Kutay, U., Bischoff, F. R., Schwamborn, K., Doenecke, D., and Görlich, D. (1999) The importin beta/importin 7 heterodimer is a functional nuclear import receptor for histone H1. The EMBO Journal 18, 2411-2423

115. Mühlhaüsser, P., Müller, E. C., Otto, A., and Kutay, U. (2001) Multiple pathways contribute to nuclear import of core histones. EMBO Reports 2, 690-696

116. Arnold, M., Nath, A., Hauber, J., and Kehlenbach, R. H. (2006) Multiple importins function as nuclear transport receptors for the Rev protein of human immunodeficiency virus type 1. The Journal of Biological Chemistry 281, 20883-20890

117. Waldmann, I., Wälde, S., and Kehlenbach, R. H. (2007) Nuclear import of c-Jun is mediated by multiple transport receptors. The Journal of Biological Chemistry 282, 27685-27692

118. Jäkel, S., and Görlich, D. (1998) Importin beta, transportin, RanBP5 and RanBP7 mediate nuclear import of ribosomal proteins in mammalian cells. The EMBO Journal 17, 4491-4502

119. Tu, L.-C., Fu, G., Zilman, A., and Musser, S. M. (2013) Large cargo transport by nuclear pores: implications for the spatial organization of FG-nucleoporins. The EMBO Journal 32, 3220-3230

120. Xu, D., Farmer, A., and Chook, Y. M. (2010) Recognition of nuclear targeting signals by Karyopherin-beta proteins. Current Opinion in Structural Biology 20, 782-790

121. Dingwall, C., Sharnick, S. V., and Laskey, R. A. (1982) A polypeptide domain that specifies migration of nucleoplasmin into the nucleus. Cell 30, 449-458

122. Lanford, R. E., and Butel, J. S. (1984) Construction and characterization of an SV40 mutant defective in nuclear transport of T antigen. Cell 37, 801-813

123. Kalderon, D., Richardson, W. D., Markham, A. F., and Smith, A. E. (1984) Sequence requirements for nuclear location of simian virus 40 large-T antigen. Nature 311, 33-38

124. Görlich, D., Kostka, S., Kraft, R., Dingwall, C., Laskey, R. A., Hartmann, E., and Prehn, S. (1995) Two different subunits of importin cooperate to recognize nuclear localization signals and bind them to the nuclear envelope. Current Biology 5, 383-392

125. Radu, A., Moore, M. S., and Blobel, G. (1995) The peptide repeat domain of nucleoporin Nup98 functions as a docking site in transport across the nuclear pore complex. Cell 81, 215-222

126. Kataoka, N., Bachorik, J. L., and Dreyfuss, G. (1999) Transportin-SR, a nuclear import receptor for SR proteins. The Journal of Cell Biology 145, 1145-1152 
127. Fischer, U., Huber, J., Boelens, W. C., Mattaj, I. W., and Lührmann, R. (1995) The HIV-1 Rev activation domain is a nuclear export signal that accesses an export pathway used by specific cellular RNAs. Cell 82, 475-483

128. Wen, W., Meinkoth, J. L., Tsien, R. Y., and Taylor, S. S. (1995) Identification of a signal for rapid export of proteins from the nucleus. Cell 82, 463-473

129. Fornerod, M., Ohno, M., Yoshida, M., and Mattaj, I. W. (1997) CRM1 is an export receptor for leucine-rich nuclear export signals. Cell 90, 1051-1060

130. Fukuda, M., Asano, S., Nakamura, T., Adachi, M., Yoshida, M., Yanagida, M., and Nishida, E. (1997) CRM1 is responsible for intracellular transport mediated by the nuclear export signal. Nature 390, 308-311

131. Sorokin, A. V., Kim, E. R., and Ovchinnikov, L. P. (2007) Nucleocytoplasmic transport of proteins. Biochemistry. Biokhimiia 72, 1439-1457

132. Poon, I. K. H., and Jans, D. A. (2005) Regulation of nuclear transport: central role in development and transformation? Traffic 6, 173-186

133. Nagase, T., Ishikawa, K., Suyama, M., Kikuno, R., Miyajima, N., Tanaka, A., Kotani, H., Nomura, N., and Ohara, O. (1998) Prediction of the coding sequences of unidentified human genes. XI. The complete sequences of 100 new cDNA clones from brain which code for large proteins in vitro. DNA Research 5, 277-286

134. Zhang, C., Sweezey, N. B., Gagnon, S., Muskat, B., Koehler, D., Post, M., and Kaplan, F. (2000) A novel karyopherin-beta homolog is developmentally and hormonally regulated in fetal lung. American Journal of Respiratory Cell and Molecular Biology 22, 451-459

135. Mingot, J. M., Kostka, S., Kraft, R., Hartmann, E., and Görlich, D. (2001) Importin 13: A novel mediator of nuclear import and export. The EMBO Journal 20, 3685-3694

136. Fatima, S., Wagstaff, K. M., Lieu, K. G., Davies, R. G., Tanaka, S. S., Yamaguchi, Y. L., Loveland, K. L., Tam, P. P. L., and Jans, D. A. (2017) Interactome of the inhibitory isoform of the nuclear transporter Importin 13. Biochimica et Biophysica Acta 1864, $546-561$

137. Ploski, J. E., Shamsher, M. K., and Radu, A. (2004) Paired-type homeodomain transcription factors are imported into the nucleus by karyopherin 13. Molecular and Cellular Biology 24, 4824-4834

138. Lin, W., Ye, W., Cai, L., Meng, X., Ke, G., Huang, C., Peng, Z., Yu, Y., Golden, J. A., Tartakoff, A. M., and Tao, T. (2009) The roles of multiple importins for nuclear import of murine aristaless-related homeobox protein. The Journal of Biological Chemistry 284, 20428-20439 
139. Shoubridge, C., Cloosterman, D., Parkinson-Lawerence, E., Brooks, D., and Gécz, J. (2007) Molecular pathology of expanded polyalanine tract mutations in the Aristalessrelated homeobox gene. Genomics 90, 59-71

140. Lin, W., Xu, P., Guo, Y., Jia, Q., and Tao, T. (2017) Nuclear import of Nkx2-2 is mediated by multiple pathways. Biochemical and Biophysical Research Communications 482, 1511-1516

141. Kahle, J., Baake, M., Doenecke, D., and Albig, W. (2005) Subunits of the heterotrimeric transcription factor NF-Y are imported into the nucleus by distinct pathways involving importin beta and importin 13. Molecular and Cellular Biology 25, 5339-5354

142. Xu, K., Tao, T., Jie, J., Lu, X., Li, X., Mehmood, M. A., He, H., Liu, Z., Xiao, X., Yang, J., Ma, J.-X., Li, W., Zhou, Y., and Liu, Z. (2013) Increased importin 13 activity is associated with the pathogenesis of pterygium. Molecular Vision 19, 604-613

143. Tao, T., Lan, J., Lukacs, G. L., Hache, R. J. G., and Kaplan, F. (2006) Importin 13 regulates nuclear import of the glucocorticoid receptor in airway epithelial cells. American Journal of Respiratory Cell and Molecular Biology 35, 668-680

144. Kanno, Y., Miyazaki, Y., and Inouye, Y. (2010) The nuclear import of the constitutive androstane receptor by importin/Ran-GTP systems. Biochimica et Biophysica Acta 1803, 968-974

145. Wang, R., Shen, J., Huang, P., and Zhu, X. (2013) CCCTC-binding factor controls its own nuclear transport via regulating the expression of importin 13. Molecules and Cells 35, 388-395

146. Kahle, J., Piaia, E., Neimanis, S., Meisterernst, M., and Doenecke, D. (2009) Regulation of nuclear import and export of negative cofactor 2. The Journal of Biological Chemistry 284, 9382-9393

147. Walker, P., Doenecke, D., and Kahle, J. (2009) Importin 13 mediates nuclear import of histone fold-containing chromatin accessibility complex heterodimers. The Journal of Biological Chemistry 284, 11652-11662

148. Liang, J., Ke, G., You, W., Peng, Z., Lan, J., Kalesse, M., Tartakoff, A. M., Kaplan, F., and Tao, T. (2008) Interaction between importin 13 and myopodin suggests a nuclear import pathway for myopodin. Molecular and Cellular Biochemistry 307, 93-100

149. Huang, S., Chang, I. S., Lin, W., Ye, W., Luo, R. Z., Lu, Z., Lu, Y., Zhang, K., Liao, W. S.-L., Tao, T., Bast, R. C., JR, Chen, X., and Yu, Y. (2009) ARHI (DIRAS3), an imprinted tumour suppressor gene, binds to importins and blocks nuclear import of cargo proteins. Bioscience Reports 30, 159-168 
150. Gontan, C., Güttler, T., Engelen, E., Demmers, J., Fornerod, M., Grosveld, F. G., Tibboel, D., Görlich, D., Poot, R. A., and Rottier, R. J. (2009) Exportin 4 mediates a novel nuclear import pathway for Sox family transcription factors. The Journal of Cell Biology 185, 27-34

151. Yoshida, K., and Blobel, G. (2001) The karyopherin Kap142p/Msn5p mediates nuclear import and nuclear export of different cargo proteins. The Journal of Cell Biology 152, 729-740

152. Blondel, M., Alepuz, P. M., Huang, L. S., Shaham, S., Ammerer, G., and Peter, M. (1999) Nuclear export of Far1p in response to pheromones requires the export receptor Msn5p/Ste21p. Genes \& Development 13, 2284-2300

153. Taberner, F. J., Quilis, I., Sendra, J., Banó, M. C., and Igual, J. C. (2012) Regulation of cell cycle transcription factor Swi5 by karyopherin Msn5. Biochimica et Biophysica Acta 1823, 959-970

154. Queralt, E., and Igual, J. C. (2003) Cell cycle activation of the Swi6p transcription factor is linked to nucleocytoplasmic shuttling. Molecular and Cellular Biology 23, 3126-3140

155. Görner, W., Durchschlag, E., Wolf, J., Brown, E. L., Ammerer, G., Ruis, H., and Schüller, C. (2002) Acute glucose starvation activates the nuclear localization signal of a stress-specific yeast transcription factor. The EMBO Journal 21, 135-144

156. Kaffman, A., Rank, N. M., O'Neill, E. M., Huang, L. S., and O'Shea, E. K. (1998) The receptor Msn5 exports the phosphorylated transcription factor Pho4 out of the nucleus. Nature 396, 482-486

157. Lipowsky, G., Bischoff, F. R., Schwarzmaier, P., Kraft, R., Kostka, S., Hartmann, E., Kutay, U., and Görlich, D. (2000) Exportin 4: a mediator of a novel nuclear export pathway in higher eukaryotes. The EMBO Journal 19, 4362-4371

158. Kurisaki, A., Kurisaki, K., Kowanetz, M., Sugino, H., Yoneda, Y., Heldin, C.-H., and Moustakas, A. (2006) The mechanism of nuclear export of Smad3 involves exportin 4 and Ran. Molecular and Cellular Biology 26, 1318-1332

159. Subramanian, K. S., Dziedzic, R. C., Nelson, H. N., Stern, M. E., Roggero, V. R., Bondzi, C., and Allison, L. A. (2015) Multiple exportins influence thyroid hormone receptor localization. Molecular and Cellular Endocrinology 411, 86-96

160. Tsuchiya, M., Ogawa, H., Suzuki, T., Sugiyama, N., Haraguchi, T., and Hiraoka, Y. (2011) Exportin 4 interacts with Sox9 through the HMG Box and inhibits the DNA binding of Sox9. PloS One 6, e25694 
161. Shoubridge, C., Tan, M. H., Fullston, T., Cloosterman, D., Coman, D., McGillivray, G., Mancini, G. M., Kleefstra, T., and Gécz, J. (2010) Mutations in the nuclear localization sequence of the Aristaless related homeobox; sequestration of mutant ARX with IPO13 disrupts normal subcellular distribution of the transcription factor and retards cell division. PathoGenetics 3, 1

162. Tao, T., Lan, J., Presley, J. F., Sweezey, N. B., and Kaplan, F. (2004) Nucleocytoplasmic shuttling of Igl2 is developmentally regulated in fetal lung. American Journal of Respiratory Cell and Molecular Biology 30, 350-359

163. Raby, B. A., van Steen, K., Lasky-Su, J., Tantisira, K., Kaplan, F., and Weiss, S. T. (2009) Importin-13 genetic variation is associated with improved airway responsiveness in childhood asthma. Respiratory Research 10, 67

164. Wang, H., Tao, T., Tang, J., Mao, Y.-H., Li, W., Peng, J., Tan, G., Zhou, Y.-P., Zhong, J.-X., Tseng, S. C. G., Kawakita, T., Zhao, Y.-X., and Liu, Z.-G. (2009) Importin 13 serves as a potential marker for corneal epithelial progenitor cells. Stem Cells 27, 2516-2526

165. You, P., Peng, Z., Wang, Y., and Tao, T. (2013) Expression and subcellular distribution of imp13 are regulated in brain development. In Vitro Cellular \& Developmental Biology. Animal 49, 346-353

166. Giagtzoglou, N., Lin, Y. Q., Haueter, C., and Bellen, H. J. (2009) Importin 13 regulates neurotransmitter release at the Drosophila neuromuscular junction. The Journal of Neuroscience 29, 5628-5639

167. Gehring, W. J. (1996) The master control gene for morphogenesis and evolution of the eye. Genes to Cells 1, 11-15

168. Callaerts, P., Halder, G., and Gehring, W. J. (1997) PAX-6 in development and evolution. Annual Review of Neuroscience 20, 483-532

169. Kitamura, K., Yanazawa, M., Sugiyama, N., Miura, H., lizuka-Kogo, A., Kusaka, M., Omichi, K., Suzuki, R., Kato-Fukui, Y., Kamiirisa, K., Matsuo, M., Kamijo, S.-i., Kasahara, M., Yoshioka, H., Ogata, T., Fukuda, T., Kondo, I., Kato, M., Dobyns, W. B., Yokoyama, M., and Morohashi, K.-i. (2002) Mutation of ARX causes abnormal development of forebrain and testes in mice and $X$-linked lissencephaly with abnormal genitalia in humans. Nature Genetics 32, 359-369

170. Friocourt, G., Poirier, K., Rakic, S., Parnavelas, J. G., and Chelly, J. (2006) The role of ARX in cortical development. The European Journal of Neuroscience 23, 869-876 
171. Quan, Y., Ji, Z.-L., Wang, X., Tartakoff, A. M., and Tao, T. (2008) Evolutionary and transcriptional analysis of karyopherin beta superfamily proteins. Molecular \& Cellular Proteomics 7, 1254-1269

172. Yamaguchi, Y. L., Tanaka, S. S., Yasuda, K., Matsui, Y., and Tam, P. P. L. (2006) Stage-specific Importin13 activity influences meiosis of germ cells in the mouse. Developmental Biology 297, 350-360

173. Zeng, B., Hu, J., Yuan, R., Hu, L., Zhong, L., and Kang, K. (2012) Increased expression of importin13 in endometriosis and endometrial carcinoma. Medical Science Monitor 18, CR361-7

174. Bannasch, D., Mehrle, A., Glatting, K.-H., Pepperkok, R., Poustka, A., and Wiemann, S. (2004) LIFEdb: a database for functional genomics experiments integrating information from external sources, and serving as a sample tracking system. Nucleic Acids Research 32, D505-8

175. Wiemann, S., Arlt, D., Huber, W., Wellenreuther, R., Schleeger, S., Mehrle, A., Bechtel, S., Sauermann, M., Korf, U., Pepperkok, R., Sültmann, H., and Poustka, A. (2004) From ORFeome to biology: A functional genomics pipeline. Genome Research 14, 2136-2144

176. Charneau, P., Mirambeau, G., Roux, P., Paulous, S., Buc, H., and Clavel, F. (1994) HIV-1 reverse transcription. A termination step at the center of the genome. Journal of Molecular Biology 241, 651-662

177. Pichler, A., Gast, A., Seeler, J. S., Dejean, A., and Melchior, F. (2002) The nucleoporin RanBP2 has SUMO1 E3 ligase activity. Cell 108, 109-120

178. Melchior, F., Sweet, D. J., and Gerace, L. (1995) Analysis of Ran/TC4 function in nuclear protein import. Methods in Enzymology 257, 279-291

179. Holden, P., and Horton, W. A. (2009) Crude subcellular fractionation of cultured mammalian cell lines. BMC Research Notes 2, 243

180. Tissera, H., Kodiha, M., and Stochaj, U. (2010) Nuclear envelopes show cell-type specific sensitivity for the permeabilization with digitonin. Protocol Exchange

181. Shevchenko, A., Wilm, M., Vorm, O., and Mann, M. (1996) Mass spectrometric sequencing of proteins silver-stained polyacrylamide gels. Analytical Chemistry 68, 850-858

182. Huang, D. W., Sherman, B. T., and Lempicki, R. A. (2009) Bioinformatics enrichment tools: paths toward the comprehensive functional analysis of large gene lists. Nucleic Acids Research 37, 1-13 
183. Huang, D. W., Sherman, B. T., and Lempicki, R. A. (2009) Systematic and integrative analysis of large gene lists using DAVID bioinformatics resources. Nature Protocols 4 , $44-57$

184. Moran, U., Phillips, R., and Milo, R. (2010) SnapShot: Key numbers in biology. Cell 141, 1262-1262

185. Riddick, G., and Macara, I. G. (2005) A systems analysis of importin-\{alpha\}-\{beta\} mediated nuclear protein import. The Journal of Cell Biology 168, 1027-1038

186. Klebe, C., Bischoff, F. R., Ponstingl, H., and Wittinghofer, A. (1995) Interaction of the nuclear GTP-binding protein Ran with its regulatory proteins RCC1 and RanGAP1. Biochemistry 34, 639-647

187. Paraskeva, E., Izaurralde, E., Bischoff, F. R., Huber, J., Kutay, U., Hartmann, E., Luhrmann, R., and Görlich, D. (1999) CRM1-mediated recycling of snurportin 1 to the cytoplasm. The Journal of Cell Biology 145, 255-264

188. Stüven, T., Hartmann, E., and Görlich, D. (2003) Exportin 6: a novel nuclear export receptor that is specific for profilin.actin complexes. The EMBO Journal 22, 5928-5940

189. Bohnsack, M. T., Regener, K., Schwappach, B., Saffrich, R., Paraskeva, E., Hartmann, E., and Görlich, D. (2002) Exp5 exports eEF1A via tRNA from nuclei and synergizes with other transport pathways to confine translation to the cytoplasm. The EMBO Journal 21, 6205-6215

190. Feng, W., Benko, A. L., Lee, J. H., Stanford, D. R., and Hopper, A. K. (1999) Antagonistic effects of NES and NLS motifs determine S. cerevisiae Rna1p subcellular distribution. Journal of Cell Science 112, 339-347

191. Plafker, K., and Macara, I. G. (2000) Facilitated Nucleocytoplasmic Shuttling of the Ran Binding Protein RanBP1. Molecular and Cellular Biology 20, 3510-3521

192. Yang, W., and Musser, S. M. (2006) Nuclear import time and transport efficiency depend on importin beta concentration. The Journal of Cell Biology 174, 951-961

193. Waldmann, I., Spillner, C., and Kehlenbach, R. H. (2012) The nucleoporin-like protein NLP1 (hCG1) promotes CRM1-dependent nuclear protein export. Journal of Cell Science 125, 144-154

194. Gill, G. (2004) SUMO and ubiquitin in the nucleus: different functions, similar mechanisms? Genes \& Development 18, 2046-2059

195. Kutay, U., Izaurralde, E., Bischoff, F. R., Mattaj, I. W., and Görlich, D. (1997) Dominant-negative mutants of importin-beta block multiple pathways of import and export through the nuclear pore complex. The EMBO Journal 16, 1153-1163 
196. Arnold, M., Nath, A., Wohlwend, D., and Kehlenbach, R. H. (2006) Transportin is a major nuclear import receptor for c-Fos: a novel mode of cargo interaction. The Journal of Biological Chemistry 281, 5492-5499

197. Chini, E. N., Chini, C. C. S., Nin, V., and Escande, C. (2013) Deleted in breast cancer-1 (DBC-1) in the interface between metabolism, aging and cancer. Bioscience Reports 33

198. Sundararajan, R., Chen, G., Mukherjee, C., and White, E. (2005) Caspasedependent processing activates the proapoptotic activity of deleted in breast cancer-1 during tumor necrosis factor-alpha-mediated death signaling. Oncogene 24, 49084920

199. Brownawell, A. M., and Macara, I. G. (2002) Exportin-5, a novel karyopherin, mediates nuclear export of double-stranded RNA binding proteins. The Journal of Cell Biology 156, 53-64

200. Mingot, J.-M., Bohnsack, M. T., Jäkle, U., and Görlich, D. (2004) Exportin 7 defines a novel general nuclear export pathway. The EMBO Journal 23, 3227-3236

201. Wang, C.-I., Chien, K.-Y., Wang, C.-L., Liu, H.-P., Cheng, C.-C., Chang, Y.-S., Yu, J.S., and Yu, C.-J. (2012) Quantitative proteomics reveals regulation of karyopherin subunit alpha-2 (KPNA2) and its potential novel cargo proteins in nonsmall cell lung cancer. Molecular \& Cellular Proteomics 11, 1105-1122

202. Zhen, Y., Sørensen, V., Skjerpen, C. S., Haugsten, E. M., Jin, Y., Wälchli, S., Olsnes, S., and Wiedlocha, A. (2012) Nuclear import of exogenous FGF1 requires the ERprotein LRRC59 and the importins Kpna1 and Kpnß1. Traffic 13, 650-664

203. Kosugi, S., Hasebe, M., Matsumura, N., Takashima, H., Miyamoto-Sato, E., Tomita, M., and Yanagawa, H. (2009) Six classes of nuclear localization signals specific to different binding grooves of importin alpha. The Journal of Biological Chemistry 284, 478-485

204. Pollard, V. W., Michael, W. M., Nakielny, S., Siomi, M. C., Wang, F., and Dreyfuss, G. (1996) A novel receptor-mediated nuclear protein import pathway. Cell 86, 985-994

205. Piñol-Roma, S., and Dreyfuss, G. (1992) Shuttling of pre-mRNA binding proteins between nucleus and cytoplasm. Nature 355, 730-732

206. Izaurralde, E., Jarmolowski, A., Beisel, C., Mattaj, I. W., Dreyfuss, G., and Fischer, U. (1997) A role for the M9 transport signal of hnRNP A1 in mRNA nuclear export. The Journal of Cell Biology 137, 27-35

207. Singh, R., and Valcárcel, J. (2005) Building specificity with nonspecific RNA-binding proteins. Nature Structural \& Molecular Biology 12, 645-653 
208. Michael, W. M., Choi, M., and Dreyfuss, G. (1995) A nuclear export signal in hnRNP A1: A signal-mediated, temperature-dependent nuclear protein export pathway. Cell 83, 415-422 


\section{List of Figures}

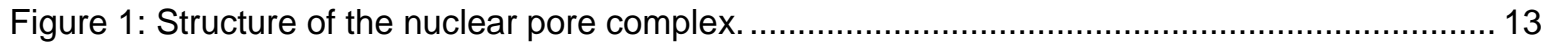

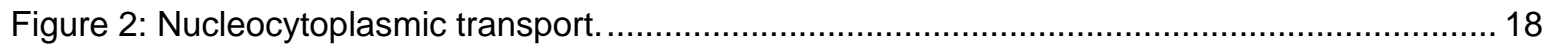

Figure 3: Structures of importin 13 import and export complexes. ........................................... 26

Figure 4: Endogenous importin 13 concentrations in cancer cell lines....................................... 75

Figure 5: Importin 13 is rate limiting for export of endogenous elF1A. ........................................ 76

Figure 6: Importin 13 promotes nuclear import of Ubc9 and nuclear export of elF1A ................... 78

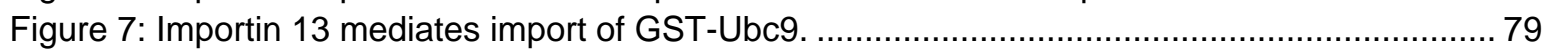

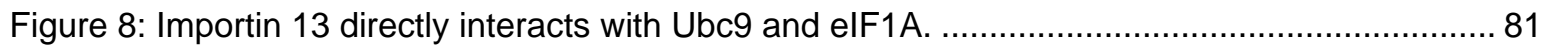

Figure 9: Importin 13 binds endogenous Ubc9 and recombinant elF1A from HeLa P4 cell extract. 83

Figure 10: Importin 13 affects the subcellular localization of DBC-1, DMAP1, TERT, DDX43,

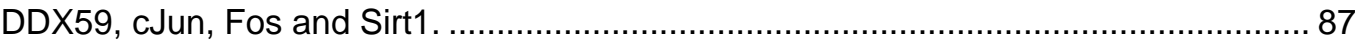

Figure 11: DBC-1, DMAP1, DDX43, DDX59 and TERT bind importin 13 differently to elF1A........ 89

Figure 12: Importin 13 interacts with the coiled-coil domain of DBC-1...................................... 90

Figure 13: The C-terminus of importin 13 is dispensable for recognition of DBC-1..................... 91

Figure 14: Proteins bound to Hzz-tag and Hzz-importin 13 from HeLa P4 cell extract. .................. 94

Figure 15: Example of importin 13 bound protein that is reduced in the presence of Ubc9........... 97

Figure 16: PPP1CB, PRMT1, CELF1, EIF4A1 and RUVBL1 change their subcellular localization

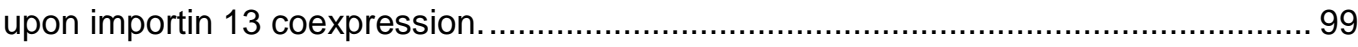

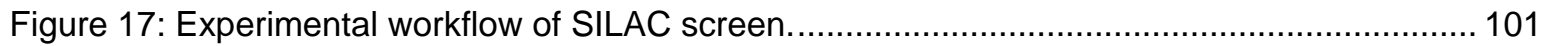

Figure 18: SILAC binding reactions and phenyl-Sepharose depletion of HeLa P4 cell extracts. ... 104

Figure 19: Putative and known importin 13 cargoes identified in SILAC screen (Imp13/Imp13+Ran vs. Imp13/Imp13+Ubc9) 106

Figure 20: Putative and known importin 13 cargoes identified in SILAC screen (Imp13+Ran/Imp13

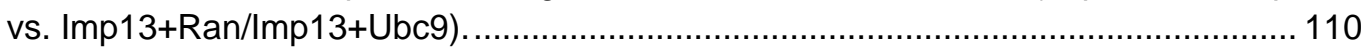

Figure 21: Importin 13 export candidates selected for further validation. .................................... 114

Figure 22: Importin 13 import candidates selected for further validation ................................... 116

Figure 23: Endogenous SILAC candidate proteins that bind to importin 13 from HeLa P4 cell extract in a RanGTP ${ }_{\mathrm{Q} 69 \mathrm{~L}}$ or Ubc9 dependent manner.

Figure 24: Effect of FLAG-importin 13 overexpression on subcellular localization of HA-tagged SILAC import cargo candidates.

Figure 25: Effect of FLAG-importin 13 overexpression on subcellular localization of GFP-GST-tagged SILAC import cargo candidates.

Figure 26: Effect of FLAG-importin 13 overexpression on subcellular localization of HA-tagged

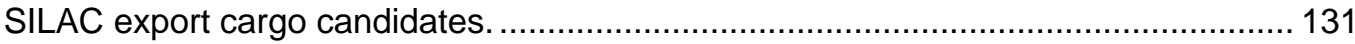

Figure 27: Effect of FLAG-importin 13 overexpression on subcellular localization of GFP-GSTtagged SILAC export cargo candidates.

Figure 28: Effect of FLAG-importin 13 overexpression on subcellular localization of HA-tagged SILAC cargo candidates in leptomycin B treated cells.

Figure 29: SILAC cargo candidates bind importin 13 differently to eIF1A and the C-terminus of importin 13 is dispensable for their interaction.

Figure S1: In situ proximity ligation assay (PLA) detection of importin 13 with Ubc9 and elF1A. .. 179

Figure S2: Proteins from the overexpression screen and other proteins analyzed for importin 13 effects.

Figure S3: SILAC binding reactions and phenyl-Sepharose depletion of HeLa P4 cell extracts (experiment 2)

Figure S4: SILAC binding reactions and phenyl-Sepharose depletion of HeLa P4 cell extracts (experiment 3) 
Figure S5: Effect of untagged importin 13 overexpression on the subcellular distribution of HA-tagged SILAC cargo candidates... 


\section{List of Tables}

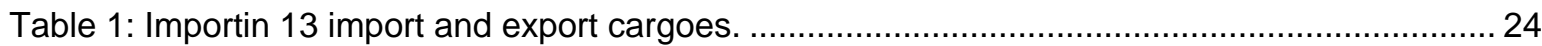

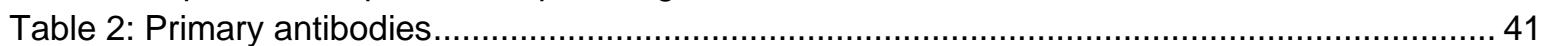

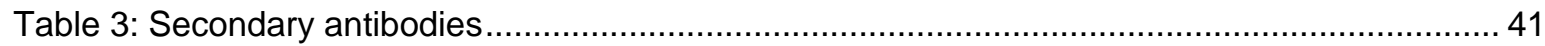

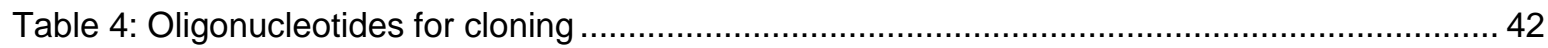

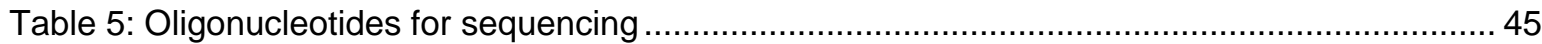

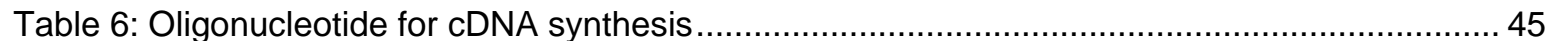

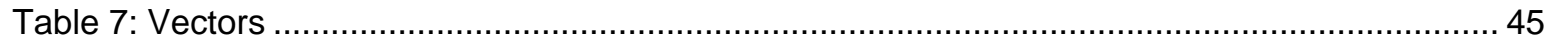

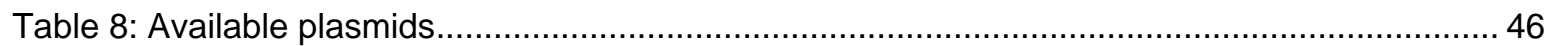

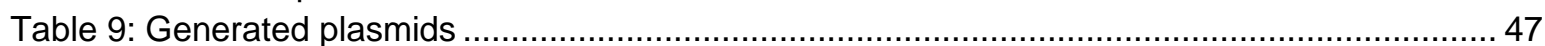

Table 10: List of proteins that bound to Hzz-importin 13 from both a digitonin and a freeze/thaw

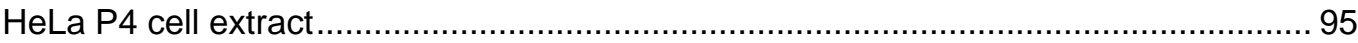

Table 11: Importin 13 bound proteins enriched from a HeLa P4 cell extract or affected by

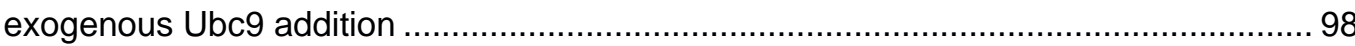

Table 12: Known importin 13 cargoes that were identified in the SILAC screen .......................... 108

Table 13: List of potential importin 13 export cargoes identified in SILAC screen that were selected

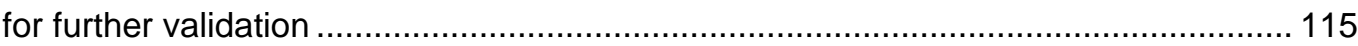

Table 14: List of potential importin 13 import cargoes identified in SILAC screen that were selected

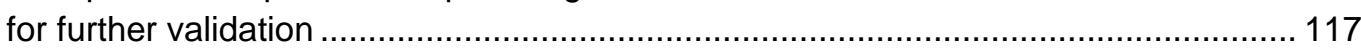

Table 15: Effect of importin 13 overexpression on importin 13 import cargo candidates identified in

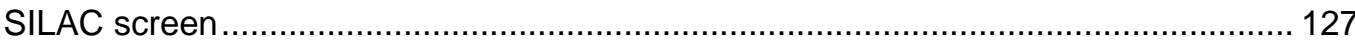

Table 16: Effect of importin 13 overexpression on importin 13 export cargo candidates identified in

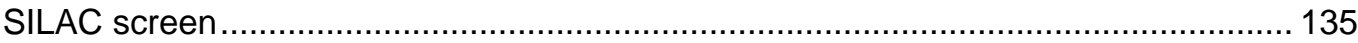

Table 17: Gene Ontology (GO) analysis of importin 13 cargo candidates ................................... 142

Table 18: Heterogeneous nuclear ribonucleoproteins (hnRNPs) identified in SILAC screen......... 155

Table S1: Proteins from overexpression screen (3.2.1) reanalyzed for changes in subcellular distribution upon importin 13 coexpression. .......................................................... 179

Table S2: List of proteins that bound to Hzz-importin 13 from a digitonin but not a freeze/thaw HeLa P4 cell extract 184

Table S3: List of proteins that bound to Hzz-importin 13 from a freeze/thaw but not a digitonin HeLa P4 cell extract 187

Table S4: Perseus workflow for the identification of importin 13 export cargoes ........................ 192

Table S5: Perseus workflow for the identification of importin 13 import cargoes ........................ 197

Table S6: List of potential importin 13 import cargoes identified in SILAC screen ...................... 200

Table S7: List of proteins identified as ambiguous importin 13 cargoes in SILAC screen............. 203

Table S8: List of potential importin 13 export cargoes identified in SILAC screen .........................203

Table S9: Importin 13 cargo candidates identified in SILAC screen and by mass spectrometry in

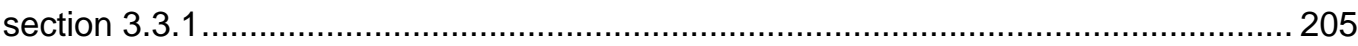

Table S10: Importin 13 cargo candidates identified in SILAC screen and the Kimura et al., 2017

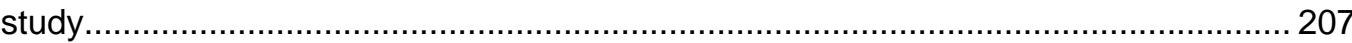

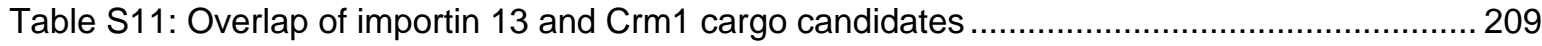

Table S12: Overlap importin 13 and importin 5 cargo candidates ............................................ 210 


\section{Appendix}
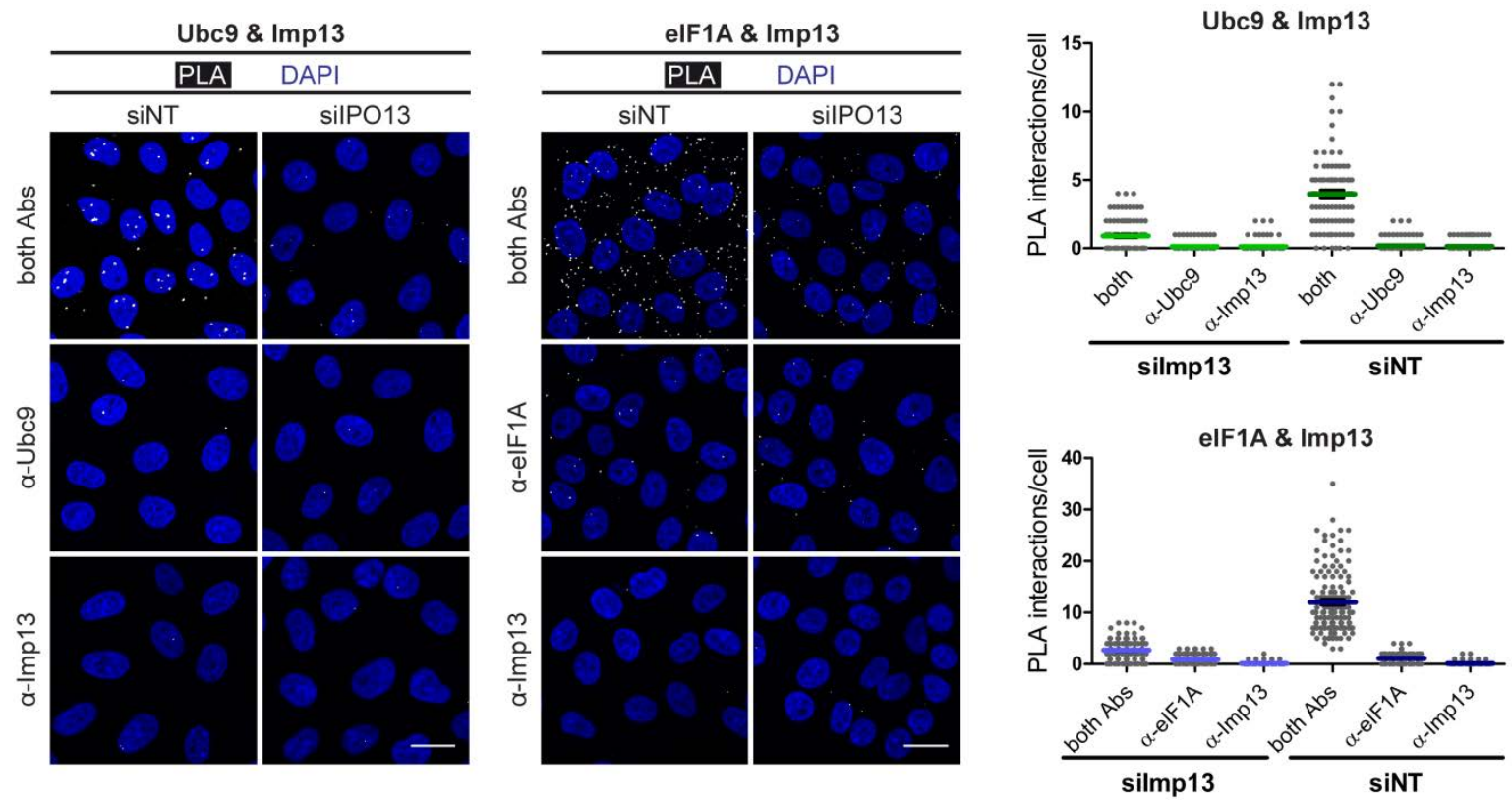

Figure S1: In situ proximity ligation assay (PLA) detection of importin 13 with Ubc9 and elF1A. Fixed HeLa P4 cells were incubated with primary antibodies against the proteins of interest, followed by incubation with secondary PLA probes. Close proximity of the PLA probes allows for rolling circle amplification, and visualization by hybridization of a fluorescent probe. Each white dot represents a single protein-protein interaction. Specificity of the observed interaction was confirmed by siRNA mediated knock-down of importin 13. A minimum of 50 cells was analyzed and PLA dots were quantified with the CellProfiler software. See section 3.1.6 for more information.

Table S1: Proteins from overexpression screen (3.2.1) reanalyzed for changes in subcellular distribution upon importin 13 coexpression.

\begin{tabular}{llc}
\hline \multicolumn{1}{c}{ Protein } & \multicolumn{1}{c}{ Protein Name } & Importin 13 Effect \\
\hline & \multicolumn{1}{c}{ Proteins from overexpression screen } \\
\hline GFP-DBC-1 & Deleted in breast cancer gene 1 & ++ \\
mCherry-DBC-1 & Deleted in breast cancer gene 1 & + \\
HA-DBC-1 & Deleted in breast cancer gene 1 & + \\
GFP-DMAP1 & DNA methyltransferase 1-associated protein 1 & ++ \\
HA-DMAP1 & DNA methyltransferase 1-associated protein 1 & + \\
GFP-TERT & Telomerase reverse transcriptase & ++ \\
TERT-HA & Telomerase reverse transcriptase & ++ \\
CFP-DDX43 & Probable ATP-dependent RNA helicase DDX43 & ++ \\
CFP-DDX59 & Probable ATP-dependent RNA helicase DDX59 & ++
\end{tabular}




\begin{tabular}{|c|c|c|}
\hline Protein & Protein Name & Importin 13 Effect \\
\hline dGFP-cJun & Transcription factor AP-1 & + \\
\hline dGFP-Fos & Proto-oncogene c-Fos & + \\
\hline Sirt1-GFP & NAD-dependent protein deacetylase sirtuin-1 & + \\
\hline Hsp22-YFP & Heat shock protein beta-8 & - \\
\hline CPF-YFP & Chondroitin polymerizing factor & - \\
\hline Lim6-YFP & LIM homeobox 6 & - \\
\hline Tmp29-YFP & Transmembrane protein 29 & - \\
\hline Nip30-YFP & NEFA-interacting nuclear protein NIP30 & - \\
\hline Zinc313-YFP & Zinc finger protein 313 & - \\
\hline KIAA1826-YFP & KIAA1826 protein & - \\
\hline DNAPTP6-YFP & DNA polymerase-transactivated protein 6 & - \\
\hline H3.3-YFP & H3 histone, family 3B & - \\
\hline \multicolumn{3}{|c|}{ Known importin 13 cargoes } \\
\hline elF1A-GFP & Eukaryotic translation initiation factor $1 \mathrm{~A}$ & ++ \\
\hline elF1A-R46E-GFP & $\begin{array}{l}\text { Eukaryotic translation initiation factor } 1 \mathrm{~A} \text { mutant } \\
\text { impaired in its interaction with importin } 13 \text { (107) }\end{array}$ & - \\
\hline HA-Ubc9 & SUMO-conjugating enzyme UBC9 & - \\
\hline GFP-GST-Ubc9 & SUMO-conjugating enzyme UBC9 & ++ \\
\hline \multicolumn{3}{|c|}{ Natural and artificial cargoes of other nuclear transport receptors } \\
\hline dGFP-GST-cNLS & $\begin{array}{l}\text { Classical nuclear localization sequence } \\
\text { (imported by importin } \alpha / \beta \text { ) }\end{array}$ & - \\
\hline GFP-M9 & $\begin{array}{l}\text { PY-NLS initially described for hnRNPA1 } \\
\text { (imported by transportin) }\end{array}$ & + \\
\hline dGFP-GST-RevNLS & $\begin{array}{l}\text { Nuclear localization signal in HIV-1Rev } \\
\text { (imported by different nuclear import receptors) }\end{array}$ & - \\
\hline HA-SPN1 & Snurportin 1 & - \\
\hline \multicolumn{3}{|c|}{ Randomly selected proteins } \\
\hline HA-p53 & Cellular tumor antigen p53 & - \\
\hline HA-ER $\alpha$ & Estrogen receptor alpha & - \\
\hline HA-Hsp70 & Heat shock protein 70 & - \\
\hline
\end{tabular}

++: strong importin 13 effect, +: weak importin 13 effect; -: no importin 13 effect 
-importin 13

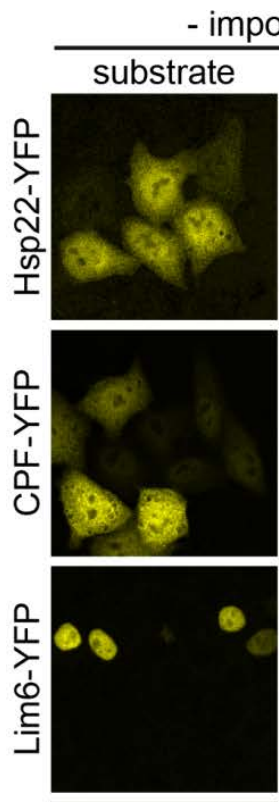

DAPI
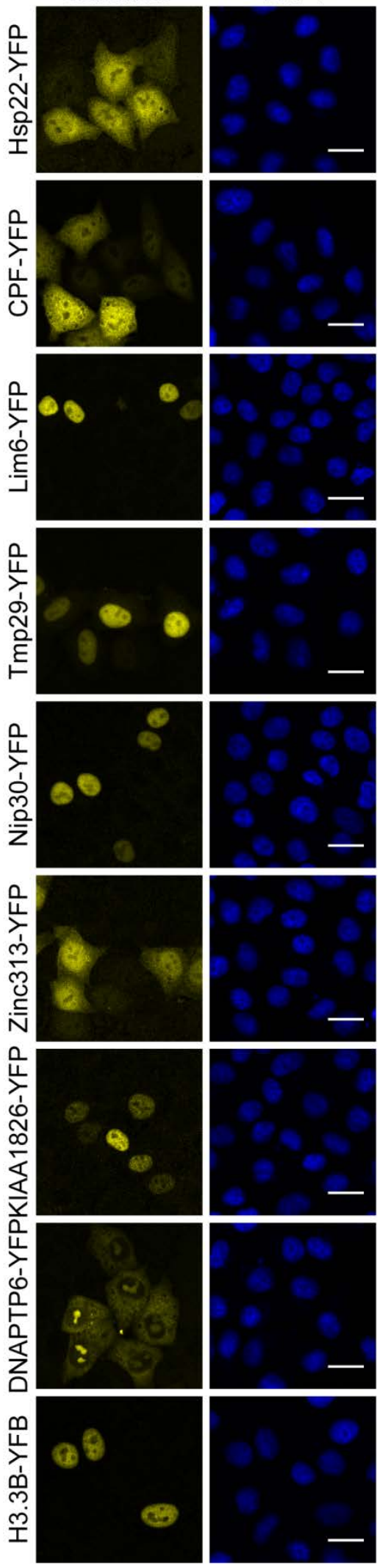

+ importin 13
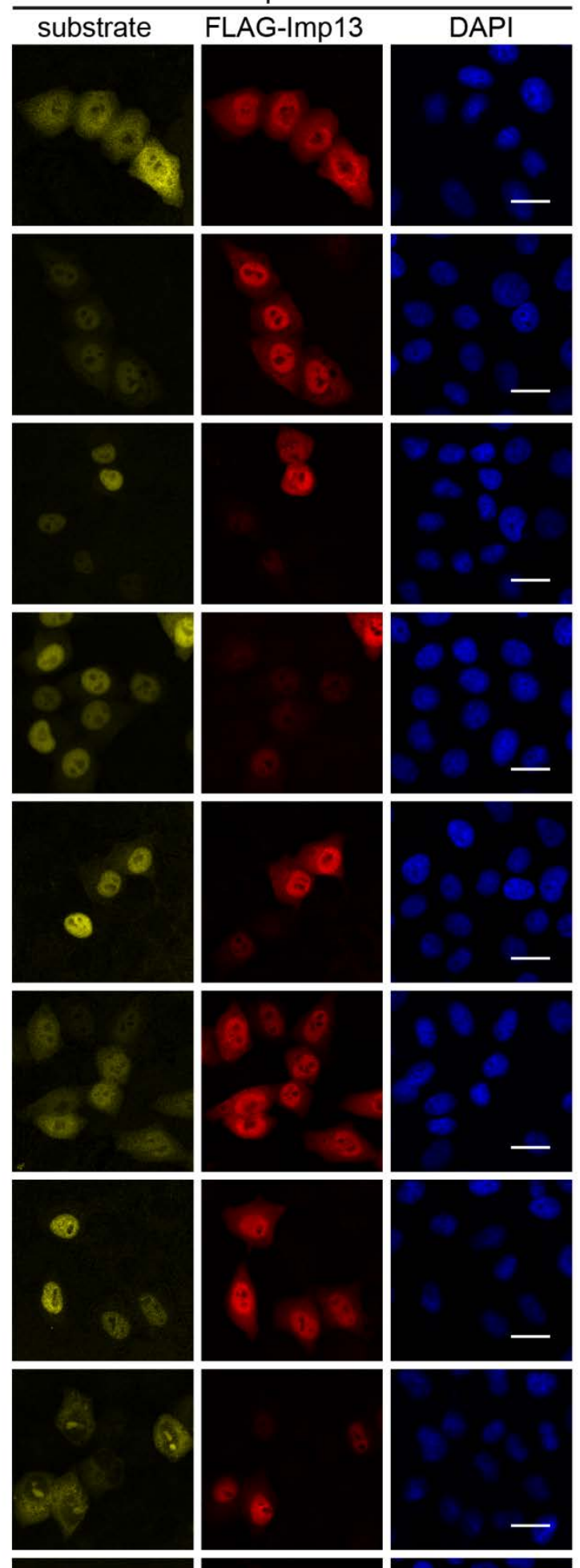

23

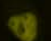

ह)
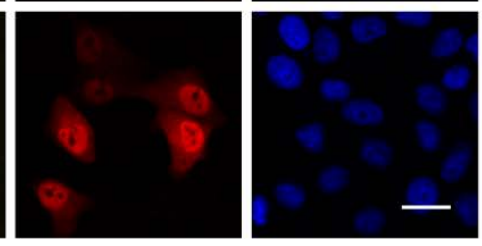

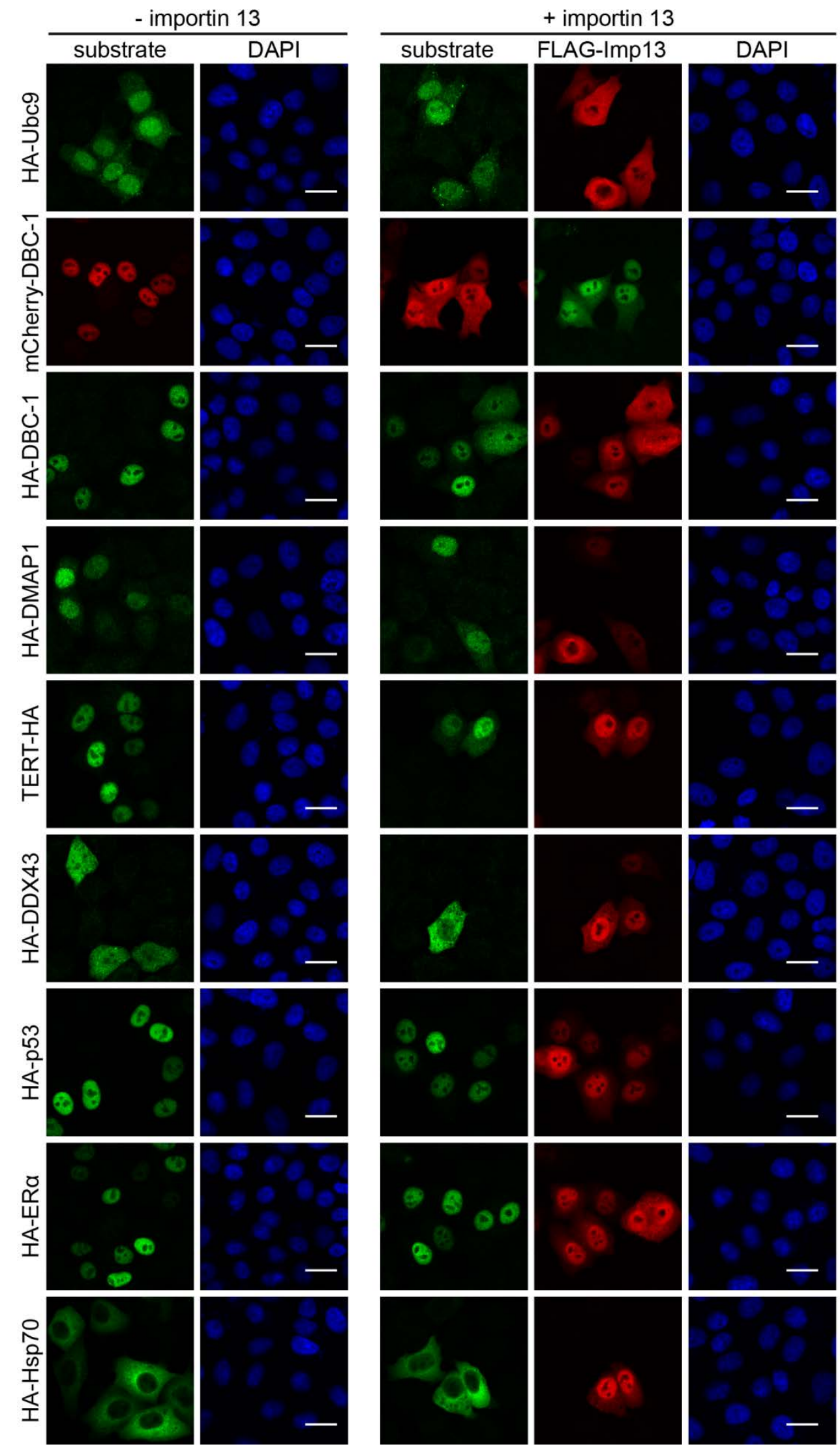


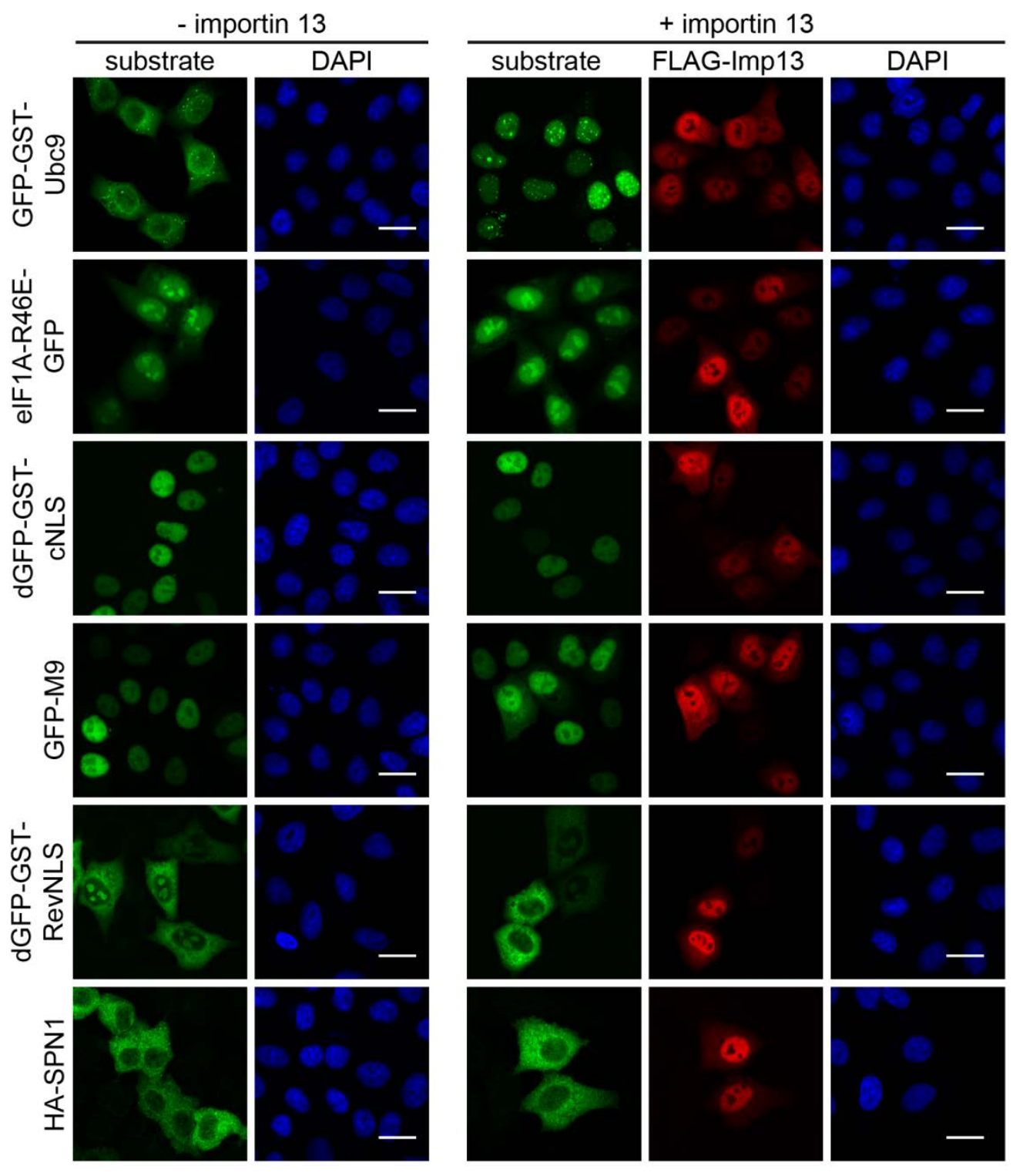

Figure S2: Proteins from the overexpression screen and other proteins analyzed for importin 13 effects. HeLa P4 cells were transiently transfected with plasmids coding for a tagged protein and an empty control vector or FLAG-importin 13 using the calcium phosphate method. The scale bars correspond to $20 \mu \mathrm{m}$. Proteins affected by importin 13 overexpression but tested with a different tag: DBC-1, DMAP1, TERT, DDX43 and DDX59. Other proteins tested from overexpression screen: Hsp22, CPF, Lim6, TMP29, Nip30, Zinc313, KIAA1826, DNAPTP6 and H3.3B. Randomly chosen proteins tested: p53, ERa and Hsp70. Artificial cargoes and proteins recognized by other nuclear transport receptors: cNLS, M9, RevNLS and SPN1. Known importin 13 cargoes: Ubc9 and elF1A. For full protein names see Table S1. See section 3.2.1 for details and Figure 10 for further proteins tested. 
Table S2: List of proteins that bound to Hzz-importin 13 from a digitonin but not a freeze/thaw HeLa P4 cell extract (see Table 10 and section 3.3.1 for details)

\begin{tabular}{|c|c|c|}
\hline Uniprot ID & Protein Name & Gene \\
\hline Q60FE5 & Filamin A & FLNA \\
\hline P68032 & Actin, alpha cardiac muscle 1 & АСТC1 \\
\hline Q71U36 & Tubulin alpha-1A chain & TUBA1A \\
\hline P49327 & Fatty acid synthase & FASN \\
\hline P12268 & Inosine-5'-monophosphate dehydrogenase 2 & IMPDH2 \\
\hline P12814 & Alpha-actinin-1 & ACTN1 \\
\hline 075369 & Filamin-B & FLNB \\
\hline Q13509 & Tubulin beta-3 chain & TUBB3 \\
\hline O15084 & Serine/threonine-protein phosphatase 6 regulatory ankyrin repeat subunit A & ANKRD28 \\
\hline Q562R1 & Beta-actin-like protein 2 & ACTBL2 \\
\hline P63244 & Receptor of activated protein C kinase 1 & RACK1 \\
\hline Q8WX93 & Palladin & PALLD \\
\hline P37802 & Transgelin-2 & TAGLN2 \\
\hline P13798 & Acylamino-acid-releasing enzyme & APEH \\
\hline Q6PGP7 & Tetratricopeptide repeat protein 37 & TTC37 \\
\hline P58107 & Epiplakin & EPPK1 \\
\hline A0A0D9SF54 & Spectrin alpha chain, non-erythrocytic 1 & SPTAN1 \\
\hline B7ZAR1 & T-complex protein 1 subunit epsilon & CCT5 \\
\hline Q8IUD2 & ELKS/Rab6-interacting/CAST family member 1 & ERC1 \\
\hline P32969 & $60 \mathrm{~S}$ ribosomal protein L9 & RPL9 \\
\hline 000743 & Serine/threonine-protein phosphatase 6 catalytic subunit & PPP6C \\
\hline Q92974 & Rho guanine nucleotide exchange factor 2 & ARHGEF2 \\
\hline P08237 & ATP-dependent 6-phosphofructokinase, muscle type & PFKM \\
\hline P34932 & Heat shock $70 \mathrm{kDa}$ protein 4 & HSPA4 \\
\hline C9JZR2 & Catenin delta-1 & CTNND1 \\
\hline P49588 & Alanine--tRNA ligase, cytoplasmic & AARS \\
\hline P52907 & F-actin-capping protein subunit alpha-1 & CAPZA1 \\
\hline P17812 & CTP synthase 1 & CTPS1 \\
\hline Q15477 & Helicase SKI2W & SKIV2L \\
\hline Q8N8S7 & Protein enabled homolog & ENAH \\
\hline P27708 & CAD protein & CAD \\
\hline P17987 & T-complex protein 1 subunit alpha & TCP1 \\
\hline Q9UQ80 & Proliferation-associated protein 2G4 & PA2G4 \\
\hline Q5T4S7 & E3 ubiquitin-protein ligase UBR4 & UBR4 \\
\hline Q8NE71 & ATP-binding cassette sub-family F member 1 & ABCF1 \\
\hline P28074 & Proteasome subunit beta type- 5 & PSMB5 \\
\hline Q96II8 & Leucine-rich repeat and calponin homology domain-containing protein 3 & $\mathrm{LRCH} 3$ \\
\hline Q01813 & ATP-dependent 6-phosphofructokinase, platelet type & PFKP \\
\hline Q7L2H7 & Eukaryotic translation initiation factor 3 subunit M & EIF3M \\
\hline Q96T76 & MMS19 nucleotide excision repair protein homolog & MMS19 \\
\hline Q96RT1 & Erbin & ERBIN \\
\hline Q9Y281 & Cofilin-2 & CFL2 \\
\hline Q01082 & Spectrin beta chain, non-erythrocytic 1 & SPTBN1 \\
\hline P39023 & $60 S$ ribosomal protein L3 & RPL3 \\
\hline Q9GZS3 & WD repeat-containing protein 61 & WDR61 \\
\hline P35579 & Myosin-9 & MYH9 \\
\hline P55884 & Eukaryotic translation initiation factor 3 subunit $B$ & EIF3B \\
\hline P14735 & Insulin-degrading enzyme & IDE \\
\hline Q15029 & $116 \mathrm{kDa}$ U5 small nuclear ribonucleoprotein component & EFTUD2 \\
\hline P05198 & Eukaryotic translation initiation factor 2 subunit 1 & EIF2S1 \\
\hline Q16513 & Serine/threonine-protein kinase N2 & PKN2 \\
\hline
\end{tabular}




\begin{tabular}{|c|c|c|}
\hline Uniprot ID & Protein Name & Gene \\
\hline Q01518 & Adenylyl cyclase-associated protein 1 & CAP1 \\
\hline 000151 & PDZ and LIM domain protein 1 & PDLIM1 \\
\hline Q9UIA9 & Exportin-7 & XPO7 \\
\hline Q92888 & Rho guanine nucleotide exchange factor 1 & ARHGEF1 \\
\hline Q96JG6 & Syndetin & VPS50 \\
\hline P61160 & Actin-related protein 2 & ACTR2 \\
\hline Q9Y2T2 & AP-3 complex subunit mu-1 & AP3M1 \\
\hline P60981 & Destrin & DSTN \\
\hline Q9Y4E8 & Ubiquitin carboxyl-terminal hydrolase 15 & USP15 \\
\hline Q7Z2W4 & Zinc finger $\mathrm{CCCH}$-type antiviral protein 1 & ZC3HAV1 \\
\hline Q96C19 & EF-hand domain-containing protein D2 & EFHD2 \\
\hline Q2NL82 & Pre-rRNA-processing protein TSR1 homolog & TSR1 \\
\hline P46060 & Ran GTPase-activating protein 1 & RANGAP1 \\
\hline O15144 & Actin-related protein $2 / 3$ complex subunit 2 & ARPC2 \\
\hline Q5VWV2 & Partitioning defective 3 homolog & PARD3 \\
\hline Q12965 & Unconventional myosin-le & MYO1E \\
\hline Q99460 & 26S proteasome non-ATPase regulatory subunit 1 & PSMD1 \\
\hline P60842 & Eukaryotic initiation factor $4 \mathrm{~A}-\mathrm{I}$ & EIF4A1 \\
\hline P62081 & $40 S$ ribosomal protein $S 7$ & RPS7 \\
\hline P47755 & F-actin-capping protein subunit alpha-2 & CAPZA2 \\
\hline Q9NYL9 & Tropomodulin-3 & TMOD3 \\
\hline Q9NZQ3 & NCK-interacting protein with $\mathrm{SH} 3$ domain & NCKIPSD \\
\hline P35268 & $60 S$ ribosomal protein L22 & RPL22 \\
\hline P47813 & Eukaryotic translation initiation factor $1 \mathrm{~A}$ & EIF1AX \\
\hline P50570 & Dynamin-2 & DNM2 \\
\hline C9J381 & Inosine-5'-monophosphate dehydrogenase & IMPDH1 \\
\hline C9J4M6 & DNA-directed RNA polymerase subunit beta & POLR2B \\
\hline A0A087WUT6 & Eukaryotic translation initiation factor 5B & EIF5B \\
\hline P61981 & $14-3-3$ protein gamma & YWHAG \\
\hline P11908 & Ribose-phosphate pyrophosphokinase 2 & PRPS2 \\
\hline Q9BVS4 & Serine/threonine-protein kinase RIO2 & RIOK2 \\
\hline P23258 & Tubulin gamma-1 chain & TUBG1 \\
\hline E9PKZO & $60 S$ ribosomal protein L8 & RPL8 \\
\hline P84077 & ADP-ribosylation factor 1 & ARF1 \\
\hline Q96LD4 & Tripartite motif-containing protein 47 & TRIM47 \\
\hline Q9NR09 & Baculoviral IAP repeat-containing protein 6 & BIRC6 \\
\hline O15371 & Eukaryotic translation initiation factor 3 subunit $D$ & EIF3D \\
\hline Q13200 & 26S proteasome non-ATPase regulatory subunit 2 & PSMD2 \\
\hline Q9UBQ5 & Eukaryotic translation initiation factor 3 subunit $\mathrm{K}$ & EIF3K \\
\hline B1ALK7 & Rho guanine nucleotide exchange factor 7 & ARHGEF7 \\
\hline Q93100 & Phosphorylase b kinase regulatory subunit beta & PHKB \\
\hline A0A087WTT1 & Polyadenylate-binding protein & PABPC1 \\
\hline Q9BTW9 & Tubulin-specific chaperone D & TBCD \\
\hline Q9BYX4 & Interferon-induced helicase $\mathrm{C}$ domain-containing protein 1 & $\mathrm{IFIH} 1$ \\
\hline Q00653 & Nuclear factor NF-kappa-B p100 subunit & NFKB2 \\
\hline Q9NR33 & DNA polymerase epsilon subunit 4 & POLE4 \\
\hline Q13347 & Eukaryotic translation initiation factor 3 subunit I & EIF3I \\
\hline P53611 & Geranylgeranyl transferase type-2 subunit beta & RABGGTB \\
\hline Q9H6S3 & Epidermal growth factor receptor kinase substrate 8 -like protein 2 & EPS8L2 \\
\hline P60228 & Eukaryotic translation initiation factor 3 subunit $E$ & EIF3E \\
\hline P62906 & $60 S$ ribosomal protein $\mathrm{L} 10 \mathrm{a}$ & RPL10A \\
\hline Q96IZ0 & PRKC apoptosis WT1 regulator protein & PAWR \\
\hline Q8N1F7 & Nuclear pore complex protein Nup93 & NUP93 \\
\hline Q14558 & Phosphoribosyl pyrophosphate synthase-associated protein 1 & PRPSAP1 \\
\hline
\end{tabular}




\begin{tabular}{|c|c|c|}
\hline Uniprot ID & Protein Name & Gene \\
\hline Q86UU1 & Pleckstrin homology-like domain family B member 1 & PHLDB1 \\
\hline O76071 & Probable cytosolic iron-sulfur protein assembly protein CIAO1 & CIAO1 \\
\hline Q92598 & Heat shock protein 105 kDa & HSPH1 \\
\hline Q5VIR6 & Vacuolar protein sorting-associated protein 53 homolog & VPS53 \\
\hline Q9Y530 & O-acetyl-ADP-ribose deacetylase 1 & OARD1 \\
\hline B1AK87 & Capping protein (Actin filament) muscle Z-line, beta & CAPZB \\
\hline P63208 & S-phase kinase-associated protein 1 & SKP1 \\
\hline P07355 & Annexin A2 & ANXA2 \\
\hline Q9BQA1 & Methylosome protein 50 & WDR77 \\
\hline P22314 & Ubiquitin-like modifier-activating enzyme 1 & UBA1 \\
\hline Q96QK1 & Vacuolar protein sorting-associated protein 35 & VPS35 \\
\hline A0A087WZK9 & Eukaryotic translation initiation factor 3 subunit $\mathrm{H}$ & EIF3H \\
\hline Q01433 & AMP deaminase 2 & AMPD2 \\
\hline P62913 & $60 S$ ribosomal protein L11 & RPL11 \\
\hline P19474 & E3 ubiquitin-protein ligase TRIM21 & TRIM21 \\
\hline Q7Z6Z7 & E3 ubiquitin-protein ligase HUWE1 & HUWE1 \\
\hline Q9UDY8 & Mucosa-associated lymphoid tissue lymphoma translocation protein 1 & MALT1 \\
\hline P21291 & Cysteine and glycine-rich protein 1 & CSRP1 \\
\hline P62753 & $40 S$ ribosomal protein 56 & RPS6 \\
\hline Q6Y7W6 & GRB10-interacting GYF protein 2 & GIGYF2 \\
\hline Q9Y4B5 & Microtubule cross-linking factor 1 & MTCL1 \\
\hline Q5T6K7 & Nuclear transcription factor $Y$ subunit gamma & NFYC \\
\hline P31946 & 14-3-3 protein beta/alpha & YWHAB \\
\hline Q15942 & Zyxin (Zyxin-2) & ZYX \\
\hline P62241 & $40 S$ ribosomal protein S8 & RPS8 \\
\hline E9PDE8 & Heat shock $70 \mathrm{kDa}$ protein $4 \mathrm{~L}$ & HSPA4L \\
\hline 000487 & 26S proteasome non-ATPase regulatory subunit 14 & PSMD14 \\
\hline Q9UPQ0 & LIM and calponin homology domains-containing protein 1 & LIMCH1 \\
\hline E7ERI8 & CLIP-associating protein 2 & CLASP2 \\
\hline P20618 & Proteasome subunit beta type-1 & PSMB1 \\
\hline A0A087X2G1 & ATP-dependent RNA helicase DDX1 & DDX1 \\
\hline Q96P70 & Importin-9 & IPO9 \\
\hline Q9Y262 & Eukaryotic translation initiation factor 3 subunit $\mathrm{L}$ & EIF3L \\
\hline Q9UNM6 & 26S proteasome non-ATPase regulatory subunit 13 & PSMD13 \\
\hline P33176 & Kinesin-1 heavy chain & KIF5B \\
\hline P10155 & 60 kDa SS-A/Ro ribonucleoprotein & TROVE2 \\
\hline Q6IBS0 & Twinfilin-2 & TWF2 \\
\hline S4R3H3 & Zinc finger SWIM domain-containing protein 8 & ZSWIM8 \\
\hline Q14258 & E3 ubiquitin/ISG15 ligase TRIM25 & TRIM25 \\
\hline Q15366 & Poly(rC)-binding protein 2 & PCBP2 \\
\hline Q14847 & LIM and SH3 domain protein 1 & LASP1 \\
\hline Q14019 & Coactosin-like protein & COTL1 \\
\hline Q15645 & Pachytene checkpoint protein 2 homolog & TRIP13 \\
\hline E9PGZ1 & Caldesmon & CALD1 \\
\hline P04075 & Fructose-bisphosphate aldolase A & ALDOA \\
\hline P62979 & Ubiquitin-40S ribosomal protein S27a & RPS27A \\
\hline Q86VP6 & Cullin-associated NEDD8-dissociated protein 1 & CAND1 \\
\hline P46778 & $60 S$ ribosomal protein L21 & RPL21 \\
\hline P25787 & Proteasome subunit alpha type-2 & PSMA2 \\
\hline Q12792 & Twinfilin-1 & TWF1 \\
\hline Q9HCN4 & GPN-loop GTPase 1 & GPN1 \\
\hline A0A024QZP7 & Cell division cycle 2 & CDC2 \\
\hline Q9NR31 & GTP-binding protein SAR1a & SAR1A \\
\hline Q02790 & Peptidyl-prolyl cis-trans isomerase FKBP4 & FKBP4 \\
\hline
\end{tabular}




\begin{tabular}{|c|c|c|}
\hline Uniprot ID & Protein Name & Gene \\
\hline K7EL20 & Eukaryotic translation initiation factor 3 subunit G & EIF3G \\
\hline P63167 & Dynein light chain 1 , cytoplasmic & DYNLL1 \\
\hline P61158 & Actin-related protein 3 & ACTR3 \\
\hline Q99471 & Prefoldin subunit 5 & PFDN5 \\
\hline P19338 & Nucleolin & $\mathrm{NCL}$ \\
\hline 000178 & GTP-binding protein 1 & GTPBP1 \\
\hline Q6NZ67 & Mitotic-spindle organizing protein 2B & MZT2B \\
\hline F8VQE1 & LIM domain and actin-binding protein 1 & LIMA1 \\
\hline Q7KZF4 & Staphylococcal nuclease domain-containing protein 1 & SND1 \\
\hline 014818 & Proteasome subunit alpha type-7 & PSMA7 \\
\hline Q12800 & Alpha-globin transcription factor CP2 & TFCP2 \\
\hline HOYM23 & Ankyrin repeat domain-containing protein 17 & ANKRD17 \\
\hline P54619 & 5'-AMP-activated protein kinase subunit gamma-1 & PRKAG1 \\
\hline P62487 & DNA-directed RNA polymerase II subunit RPB7 & POLR2G \\
\hline P17858 & ATP-dependent 6-phosphofructokinase, liver type & PFKL \\
\hline Q86TC9 & Myopalladin & MYPN \\
\hline D6REY2 & Colorectal mutant cancer protein & MCC \\
\hline Q9Y3F4 & Serine-threonine kinase receptor-associated protein & STRAP \\
\hline Q5VZK9 & F-actin-uncapping protein LRRC16A & CARMIL1 \\
\hline Q5VYK3 & Proteasome-associated protein ECM29 homolog & ECM29 \\
\hline E5RHG8 & Elongin-C & ELOC \\
\hline 075340 & Programmed cell death protein 6 & PDCD6 \\
\hline Q9Y597 & BTB/POZ domain-containing protein KCTD3 & KCTD3 \\
\hline Q96CW5 & Gamma-tubulin complex component 3 & TUBGCP3 \\
\hline $\mathrm{F} 5 \mathrm{H} 018$ & GTP-binding nuclear protein Ran & RAN \\
\hline Q9UDY4 & DnaJ homolog subfamily B member 4 & DNAJB4 \\
\hline Q9BV44 & THUMP domain-containing protein 3 & THUMPD3 \\
\hline
\end{tabular}

\#: Proteins are sorted according to the total number of identified peptide sequences for a protein. Proteins written in bold are known importin 13 cargoes. Identified proteins that corresponded to the same protein but had different Uniprot IDs were listed only once. Further keratin and immunoglobulin contaminants were removed.

Table S3: List of proteins that bound to Hzz-importin 13 from a freeze/thaw but not a digitonin HeLa P4 cell extract (see Table 10 and section 3.3.1 for details) ${ }^{\#}$

\begin{tabular}{lll}
\hline Uniprot ID & Protein Name & Gene \\
\hline A0A0U1RQF0 & Fatty acid synthase & FASN \\
Q9BPW8 & Protein NipSnap homolog 1 & NIPSNAP1 \\
O75323 & Protein NipSnap homolog 2 & NIPSNAP2 \\
P39880 & Homeobox protein cut-like 1 & CUX1 \\
C9JE98 & Nuclear receptor corepressor 2 & NCOR2 \\
Q5HY54 & Filamin-A & FLNA \\
O75376 & Nuclear receptor corepressor 1 & NCOR1 \\
Q13952 & Nuclear transcription factor Y subunit gamma & NFYC \\
P40424 & Pre-B-cell leukemia transcription factor 1 & PBX1 \\
A0A087WZ13 & Ribonucleoprotein PTB-binding 1 & RAVER1 \\
K7EKE6 & Lon protease homolog, mitochondrial & LONP1 \\
P25208 & Nuclear transcription factor Y subunit beta & NFYB \\
Q14919 & Dr1-associated corepressor & DRAP1 \\
Q9Y6D9 & Mitotic spindle assembly checkpoint protein MAD1 & MAD1L1 \\
Q01658 & Protein Dr1 & DR1
\end{tabular}




\begin{tabular}{|c|c|c|}
\hline Uniprot ID & Protein Name & Gene \\
\hline P62841 & $40 S$ ribosomal protein S15 & RPS15 \\
\hline P40425 & Pre-B-cell leukemia transcription factor 2 & PBX2 \\
\hline 075116 & Rho-associated protein kinase 2 & ROCK2 \\
\hline A0A0C4DFV9 & Protein SET & SET \\
\hline B1AKN7 & Nuclear factor 1 & NFIA \\
\hline P49321 & Nuclear autoantigenic sperm protein & NASP \\
\hline Q8TEW0 & Partitioning defective 3 homolog & PARD3 \\
\hline F5GYS8 & Homeobox protein Meis1 & MEIS1 \\
\hline O14770 & Homeobox protein Meis2 & MEIS2 \\
\hline 095425 & Supervillin & SVIL \\
\hline P08651 & Nuclear factor 1 C-type & NFIC \\
\hline Q92734 & Protein TFG & TFG \\
\hline P62854 & $40 S$ ribosomal protein S26 & RPS26 \\
\hline Q14444 & Caprin-1 & CAPRIN1 \\
\hline 060506 & Heterogeneous nuclear ribonucleoprotein Q & SYNCRIP \\
\hline Q5VTR2 & E3 ubiquitin-protein ligase BRE1A & RNF20 \\
\hline Q15120 & [Pyruvate dehydrogenase & PDK3 \\
\hline P35249 & Replication factor $\mathrm{C}$ subunit 4 & RFC4 \\
\hline Q8NHP8 & Putative phospholipase B-like 2 & PLBD2 \\
\hline H9KV28 & Protein diaphanous homolog 1 & DIAPH1 \\
\hline J3QL15 & Ribosomal protein L19 & RPL19 \\
\hline HOYNH8 & Uveal autoantigen with coiled-coil domains and ankyrin repeats & UACA \\
\hline P62750 & $60 S$ ribosomal protein $\mathrm{L} 23 \mathrm{a}$ & RPL23A \\
\hline Q09666 & Neuroblast differentiation-associated protein AHNAK & AHNAK \\
\hline Q8TF72 & Protein Shroom3 & SHROOM3 \\
\hline Q13162 & Peroxiredoxin-4 & PRDX4 \\
\hline I3L2J8 & Centrosomal protein of $131 \mathrm{kDa}$ & CEP131 \\
\hline C9JXB8 & $60 S$ ribosomal protein L24 & RPL24 \\
\hline P26373 & $60 S$ ribosomal protein L13 & RPL13 \\
\hline Q99536 & Synaptic vesicle membrane protein VAT-1 homolog & VAT1 \\
\hline P78337 & Pituitary homeobox 1 & PITX1 \\
\hline P48634 & Protein PRRC2A & PRRC2A \\
\hline P60953 & Cell division control protein 42 homolog & CDC42 \\
\hline Q14678 & $\mathrm{KN}$ motif and ankyrin repeat domain-containing protein 1 & KANK1 \\
\hline Q96A19 & Coiled-coil domain-containing protein 102A & CCDC102A \\
\hline Q9H2J4 & Phosducin-like protein 3 & PDCL3 \\
\hline P62826 & GTP-binding nuclear protein Ran & RAN \\
\hline P85037 & Forkhead box protein $\mathrm{K} 1$ & FOXK1 \\
\hline R4GNB2 & DENN domain-containing protein $4 \mathrm{C}$ & DENND4C \\
\hline Q13136 & Liprin-alpha-1 & PPFIA1 \\
\hline Q9Y2R9 & $28 \mathrm{~S}$ ribosomal protein $\mathrm{S} 7$, mitochondrial & MRPS7 \\
\hline H7C2W9 & $60 S$ ribosomal protein L31 & RPL31 \\
\hline A6NJA2 & Ubiquitin carboxyl-terminal hydrolase 14 & USP14 \\
\hline
\end{tabular}

\#: Proteins are sorted according to the total number of identified peptide sequences for a protein. Proteins written in bold are known importin 13 cargoes. Identified proteins that corresponded to the same protein but had different Uniprot IDs were listed only once. Further keratin and immunoglobulin contaminants were removed. 
A SILAC binding reactions:

1. Hzz-importin 13 + light HeLa P4 cell extract (I) + RanGTP ${ }_{\text {Q69L }}$

2. Hzz-importin $13+$ medium HeLa P4 cell extract (m)

3. Hzz-importin $13+$ heavy HeLa P4 cell extract $(h)+$ Ubc9

B

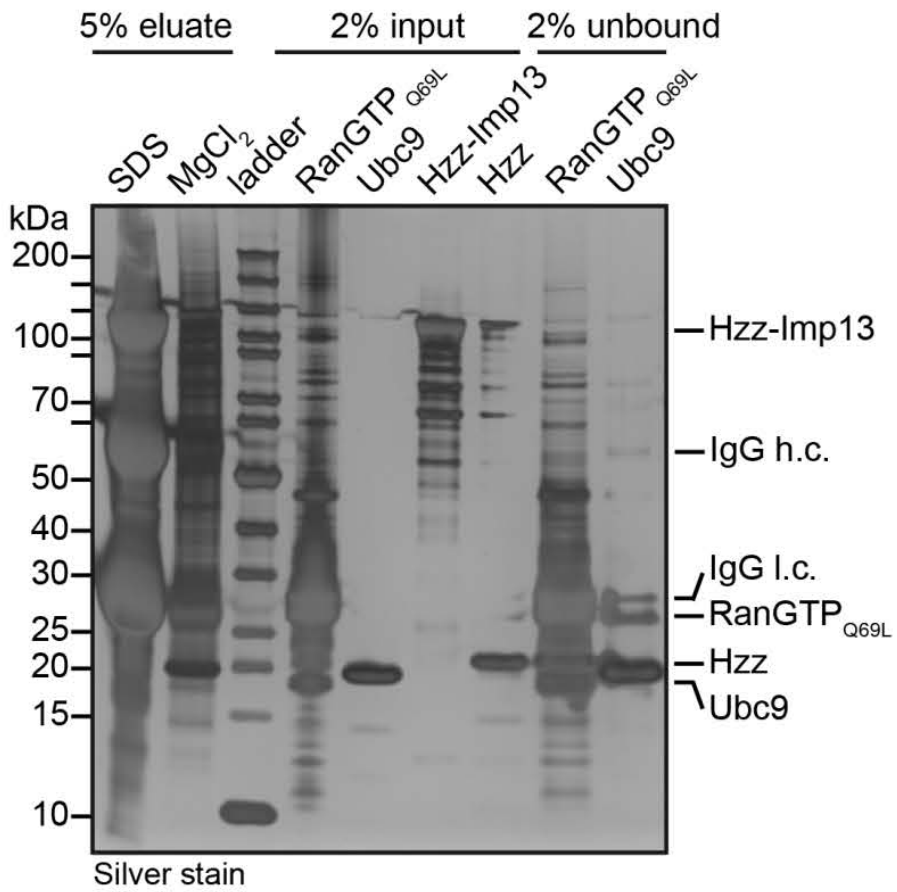

C

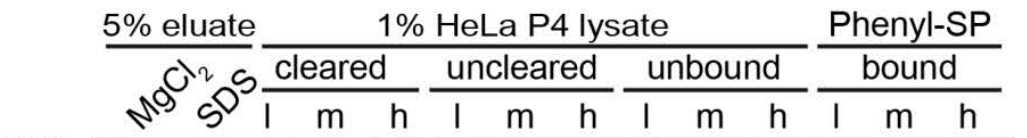

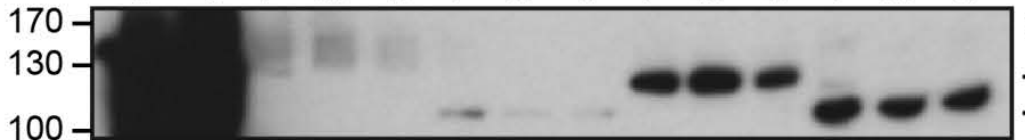

a-Imp13

100

130

-

- Hzz-Imp13

- endo. Imp13

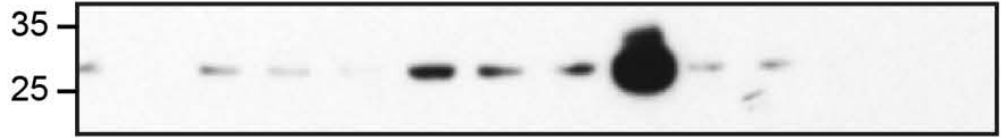

a-Ran

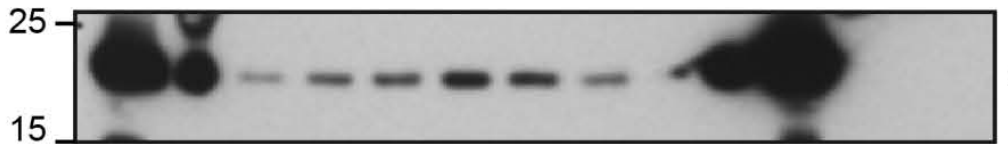

$\alpha-U b c 9$

25

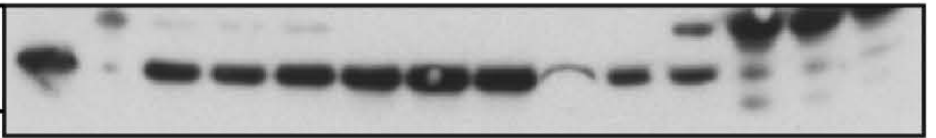

a-elF1A 
Figure S3: SILAC binding reactions and phenyl-Sepharose depletion of HeLa P4 cell extracts (experiment 2). (A) Schematic showing exemplary SILAC binding reactions. (B, C) Hzz-tagged importin $13(0.5 \mathrm{nmol})$ was immobilized on IgG-Sepharose and incubated with cell extracts of HeLa P4 cells grown in DMEM medium containing light, medium or heavy amino acids in the absence or presence of $10 \mu \mathrm{M}$ RanGTP ${ }_{\mathrm{Q} 69 \mathrm{~L}}$ or $5 \mu \mathrm{M}$ Ubc9 in transport buffer. Cell extracts (uncleared) were precleared with Hzz/lgG-Sepharose and phenyl-Sepharose (cleared) to reduce unspecific interactions. Bound proteins were eluted in a first elution step with magnesium chloride, followed by a second elution step with $4 x$ SDS sample buffer. Cell lysates and eluted proteins were separated by SDS-PAGE and analyzed by silver staining (B) or immunoblotting with an anti-importin 13 , antitransportin, anti-importin $\beta$, anti-Ran, anti-Ubc9 and anti-elF1A antibody (C). Note that in the unbound cell lysate some free $\mathrm{Hzz}$-importin 13 can be detected, as well as excess exogenous RanGTP $_{\mathrm{Q} 69 \mathrm{~L}}$ and Ubc9 in the unbound light and heavy isotopically labeled cell lysate, respectively. See section 3.3.3 for details and Figure 18 and Figure S4 for replicates. ladder: PageRuler Unstained Protein Ladder; $\mathrm{MgCl}_{2}$ : magnesium chloride eluate; SDS: 4x SDS sample buffer eluate; I: light; m: medium; h: heavy; endog.: endogenous. 
A SILAC binding reactions:

1. Hzz-importin 13 + light HeLa P4 cell extract (I) + RanGTP ${ }_{\text {Q69L }}$

2. Hzz-importin $13+$ medium HeLa P4 cell extract $(m)$

3. Hzz-importin $13+$ heavy HeLa P4 cell extract $(h)+$ Ubc9

B

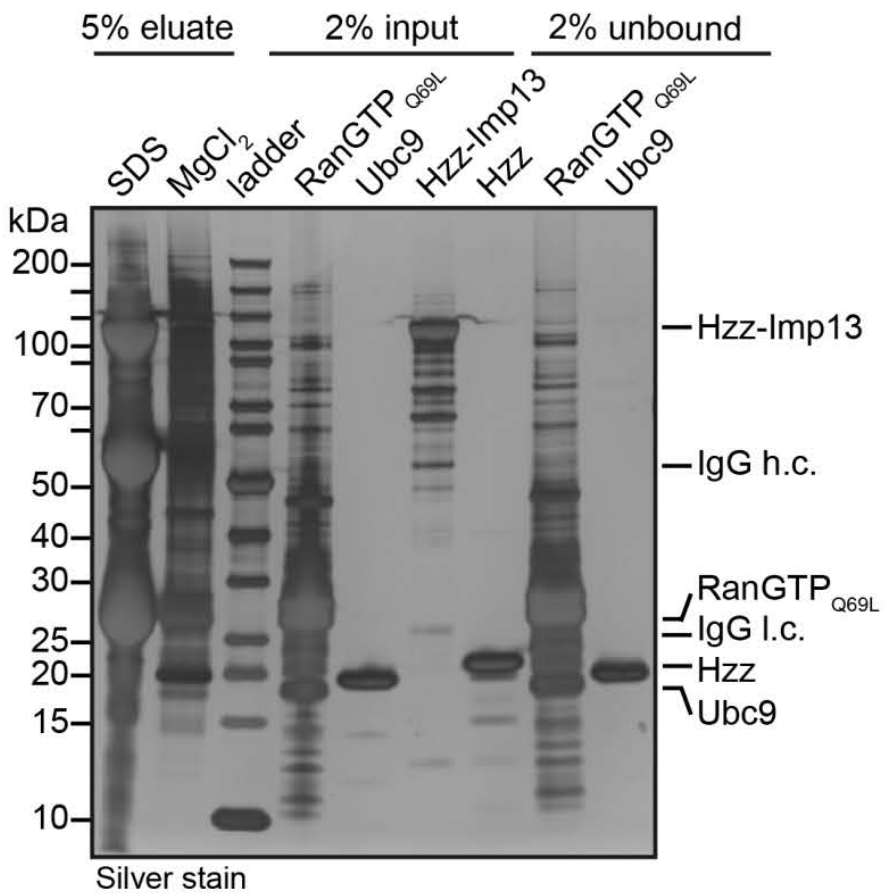

C

$5 \%$ eluate

$1 \%$ HeLa P4 lysate

Phenyl-SP $\mathrm{c}^{2} \mathrm{~s}$ cleared uncleared unbound bound
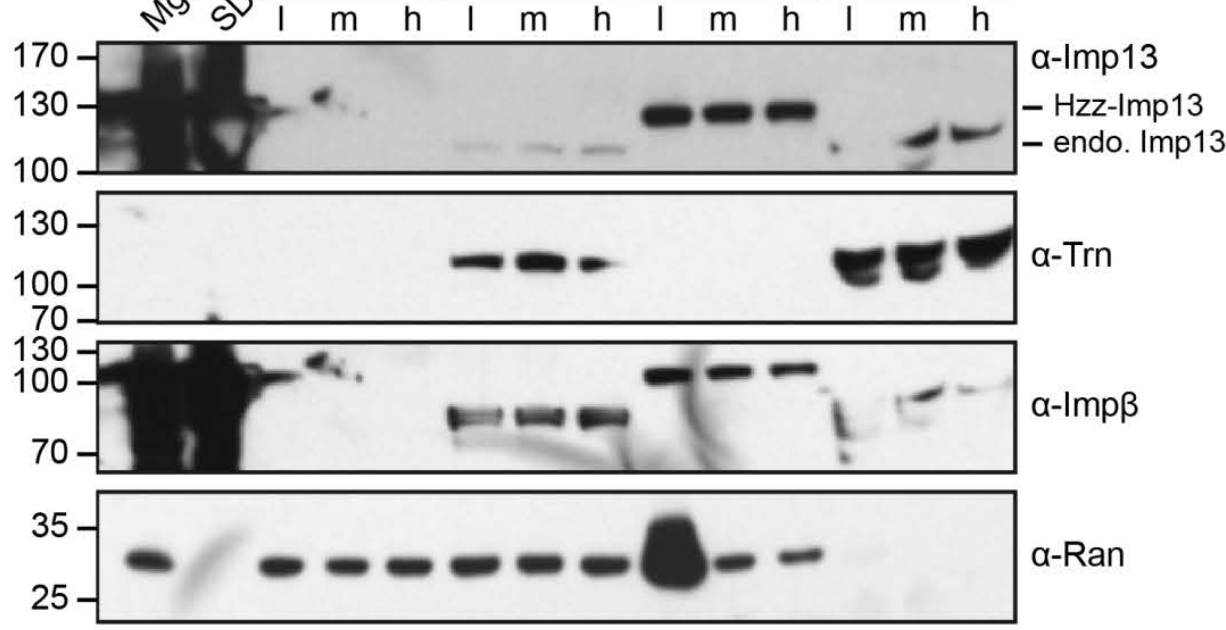

a-Ran

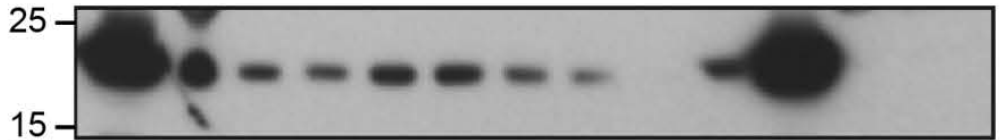

$\alpha-U b c 9$

25

15

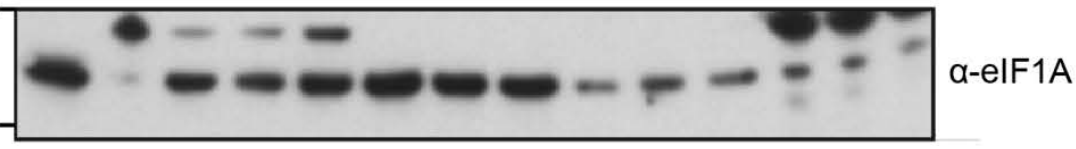


Figure S4: SILAC binding reactions and phenyl-Sepharose depletion of HeLa P4 cell extracts (experiment 3). (A) Schematic showing exemplary SILAC binding reactions. (B, C) Hzz-tagged importin $13(0.5 \mathrm{nmol})$ was immobilized on IgG-Sepharose and incubated with cell extracts of HeLa P4 cells grown in DMEM medium containing light, medium or heavy amino acids in the absence or presence of $10 \mu \mathrm{M}$ RanGTP ${ }_{\mathrm{Q} 69 \mathrm{~L}}$ or $5 \mu \mathrm{M}$ Ubc9 in transport buffer. Cell extracts (uncleared) were precleared with Hzz/lgG-Sepharose and phenyl-Sepharose (cleared) to reduce unspecific interactions. Bound proteins were eluted in a first elution step with magnesium chloride, followed by a second elution step with $4 x$ SDS sample buffer. Cell lysates and eluted proteins were separated by SDS-PAGE and analyzed by silver staining (B) or immunoblotting with an anti-importin 13, antitransportin, anti-importin $\beta$, anti-Ran, anti-Ubc9 and anti-eIF1A antibody (C). Note that in the unbound cell lysate some free Hzz-importin 13 can be detected, as well as excess exogenous RanGTP $_{\mathrm{Q} 69 \mathrm{~L}}$ and Ubc9 in the unbound light and heavy isotopically labeled cell lysate, respectively. See section 3.3.3 for details and Figure 18 and Figure S3 for replicates. ladder: PageRuler Unstained Protein Ladder; $\mathrm{MgCl}_{2}$ : magnesium chloride eluate; SDS: 4x SDS sample buffer eluate; I: light; m: medium; h: heavy; endog.: endogenous.

Table S4: Perseus workflow for the identification of importin 13 export cargoes (see section 3.3.3 for SILAC experiment)

\begin{tabular}{|c|c|c|c|}
\hline Matrix & Processing & Settings & Description \\
\hline Matrix 1 & $\begin{array}{l}\text { Generic matrix } \\
\text { upload }\end{array}$ & $\begin{array}{l}\text { Expression: } \\
\text { Ratio M/L normalized SILAC4 } \\
\text { Ratio H/L normalized SILAC4 } \\
\text { Ratio H/M normalized SILAC4 } \\
\text { Ratio M/L normalized SILAC5 } \\
\text { Ratio H/L normalized SILAC5 } \\
\text { Ratio H/M normalized SILAC5 } \\
\text { Ratio M/L normalized SILAC6 } \\
\text { Ratio H/L normalized SILAC6 } \\
\text { Ratio H/M normalized SILAC6 } \\
\text { Numerical: } \\
\text { Ratio M/L variability [\%] SILAC4 } \\
\text { Ratio H/L variability [\%] SILAC4 } \\
\text { Ratio H/M variability [\%] SILAC4 } \\
\text { Ratio M/L variability [\%] SILAC5 } \\
\text { Ratio H/L variability [\%] SILAC5 } \\
\text { Ratio H/M variability [\%] SILAC5 } \\
\text { Ratio M/L variability [\%] SILAC6 } \\
\text { Ratio H/L variability [\%] SILAC6 } \\
\text { Ratio H/M variability [\%] SILAC6 } \\
\text { Ratio M/L count SILAC4 } \\
\text { Ratio H/L count SILAC4 } \\
\text { Ratio H/M count SILAC4 } \\
\text { Ratio M/L count SILAC5 } \\
\text { Ratio H/L count SILAC5 } \\
\text { Ratio H/M count SILAC5 } \\
\text { Ratio M/L count SILAC6 } \\
\text { Ratio H/L count SILAC6 } \\
\text { Ratio H/M count SILAC6 } \\
\text { Categorical: } \\
\text { Only identified by site } \\
\text { Reverse } \\
\text { Potential contaminants } \\
\text { Text: } \\
\text { Protein IDs }\end{array}$ & $\begin{array}{l}\text { Load MaxQuant derived proteomics } \\
\text { data from the tab-separated file } \\
\text { proteinGroups.txt }\end{array}$ \\
\hline
\end{tabular}


Appendix

\begin{tabular}{|c|c|c|c|}
\hline Matrix & Processing & Settings & Description \\
\hline & & $\begin{array}{l}\text { Majority protein IDs } \\
\text { Protein names } \\
\text { Gene names }\end{array}$ & \\
\hline Matrix 2 & $\begin{array}{l}\text { Filter rows based on } \\
\text { categorical column }\end{array}$ & $\begin{array}{l}\text { Column: Only identified by site } \\
\text { Values: + } \\
\text { Mode: Remove matching rows } \\
\text { Filter mode: Reduce matrix }\end{array}$ & $\begin{array}{l}\text { Remove proteins only identified by } \\
\text { peptides carrying a modified residue }\end{array}$ \\
\hline Matrix 3 & $\begin{array}{l}\text { Filter rows based on } \\
\text { categorical column }\end{array}$ & $\begin{array}{l}\text { Column: Reverse } \\
\text { Values: + } \\
\text { Mode: Remove matching rows } \\
\text { Filter mode: Reduce matrix }\end{array}$ & $\begin{array}{l}\text { Remove hits that match against a } \\
\text { nonsense database }\end{array}$ \\
\hline Matrix 4 & $\begin{array}{l}\text { Filter rows based on } \\
\text { categorical column }\end{array}$ & $\begin{array}{l}\text { Column: Potential contaminants } \\
\text { Values: + } \\
\text { Mode: Remove matching rows } \\
\text { Filter mode: Reduce matrix }\end{array}$ & Remove common contaminants \\
\hline Matrix 5 & $\begin{array}{l}\text { Remove empty } \\
\text { columns }\end{array}$ & & Remove empty columns \\
\hline Matrix 6 & $\begin{array}{l}\text { Select rows } \\
\text { manually }\end{array}$ & $\begin{array}{l}\text { Select immunoglobulin contaminants } \\
\text { manually and remove selected rows }\end{array}$ & $\begin{array}{l}\text { Remove immunoglobulin } \\
\text { contaminants }\end{array}$ \\
\hline Matrix 7 & Transform & $\begin{array}{l}\text { Transformation: } 1 / x \\
\text { Columns: } \\
\text { Ratio M/L normalized SILAC4 } \\
\text { Ratio H/L normalized SILAC4 } \\
\text { Ratio H/M normalized SILAC4 } \\
\text { Ratio } \mathrm{M} / \mathrm{L} \text { normalized SILAC5 } \\
\text { Ratio H/L normalized SILAC5 } \\
\text { Ratio H/M normalized SILAC5 } \\
\text { Ratio } \mathrm{M} / \mathrm{L} \text { normalized SILAC6 } \\
\text { Ratio H/L normalized SILAC6 } \\
\text { Ratio H/M normalized SILAC6 }\end{array}$ & Invert ratios by using the formula $1 / x$ \\
\hline Matrix 8 & $\begin{array}{l}\text { Matching rows by } \\
\text { name }\end{array}$ & $\begin{array}{l}\text { Base matrix: Matrix } 6 \\
\text { Other matrix: Matrix } 7 \\
\text { Matching column 1: Protein IDs } \\
\text { Matching column 2: Protein IDs } \\
\text { Expression columns: } \\
\text { Ratio M/L normalized SILAC4 } \\
\text { Ratio H/L normalized SILAC4 } \\
\text { Ratio H/M normalized SILAC4 } \\
\text { Ratio M/L normalized SILAC5 } \\
\text { Ratio H/L normalized SILAC5 } \\
\text { Ratio H/M normalized SILAC5 } \\
\text { Ratio M/L normalized SILAC6 } \\
\text { Ratio H/L normalized SILAC6 } \\
\text { Ratio H/M normalized SILAC6 }\end{array}$ & $\begin{array}{l}\text { Combine columns of the existing } \\
\text { protein ratios with the newly } \\
\text { generated inverted ratios (rows of the } \\
\text { other matrix are associated with rows } \\
\text { of the base matrix via matching } \\
\text { expressions in a textual column from } \\
\text { each matrix) }\end{array}$ \\
\hline
\end{tabular}


Appendix

\begin{tabular}{|c|c|c|c|}
\hline Matrix & Processing & Settings & Description \\
\hline Matrix 9 & Transform & $\begin{array}{l}\text { Transformation: log2(x) } \\
\text { Columns: } \\
\text { Ratio M/L normalized SILAC4 } \\
\text { Ratio H/L normalized SILAC4 } \\
\text { Ratio H/M normalized SILAC4 } \\
\text { Ratio M/L normalized SILAC5 } \\
\text { Ratio H/L normalized SILAC5 } \\
\text { Ratio H/M normalized SILAC5 } \\
\text { Ratio M/L normalized SILAC6 } \\
\text { Ratio H/L normalized SILAC6 } \\
\text { Ratio H/M normalized SILAC6 } \\
\text { Ratio M/L normalized SILAC4_1 } \\
\text { Ratio H/L normalized SILAC4_1 } \\
\text { Ratio H/M normalized SILAC4_1 } \\
\text { Ratio M/L normalized SILAC5_1 } \\
\text { Ratio H/L normalized SILAC5_1 } \\
\text { Ratio H/M normalized SILAC5_1 } \\
\text { Ratio M/L normalized SILAC6_1 } \\
\text { Ratio H/L normalized SILAC6_1 } \\
\text { Ratio H/M normalized SILAC6_1 }\end{array}$ & $\begin{array}{l}\text { Log transformation of expression } \\
\text { columns using the formula log2(x) }\end{array}$ \\
\hline Matrix 10 & $\begin{array}{l}\text { Categorical } \\
\text { annotation rows }\end{array}$ & 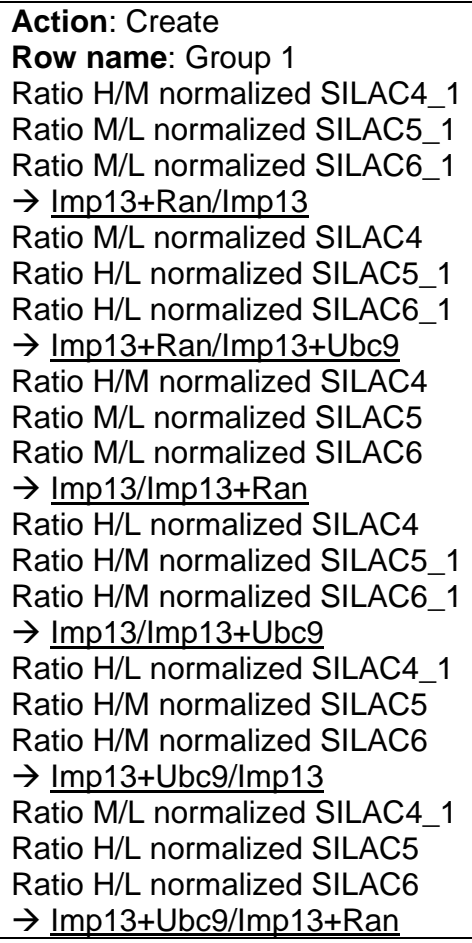 & $\begin{array}{l}\text { Define the following ratio groups: } \\
\text { Imp13+Ran/Imp13 } \\
\text { Imp13+Ran/Imp13+Ubc9 } \\
\text { Imp13/Imp13+Ran } \\
\text { Imp13/Imp13+Ubc9 } \\
\text { Imp13+Ubc9/Imp13 } \\
\text { Imp13+Ubc9/Imp13+Ran }\end{array}$ \\
\hline
\end{tabular}




\begin{tabular}{|c|c|c|c|}
\hline Matrix & Processing & Settings & Description \\
\hline Matrix 11 & $\begin{array}{l}\text { Reorder/remove } \\
\text { columns }\end{array}$ & $\begin{array}{l}\text { Expression column: } \\
\text { Ratio H/M normalized SILAC4_1 } \\
\text { Ratio M/L normalized SILAC5_1 } \\
\text { Ratio M/L normalized SILAC6_1 } \\
\text { Ratio M/L normalized SILAC4 } \\
\text { Ratio H/L normalized SILAC5_1 } \\
\text { Ratio H/L normalized SILAC6_1 } \\
\text { Numerical columns: } \\
\text { Ratio } \mathrm{M} / \mathrm{L} \text { variability [\%] SILAC4 } \\
\text { Ratio H/M variability [\%] SILAC4 } \\
\text { Ratio M/L variability [\%] SILAC5 } \\
\text { Ratio H/L variability [\%] SILAC5 } \\
\text { Ratio } \mathrm{M} / \mathrm{L} \text { variability [\%] SILAC6 } \\
\text { Ratio H/L variability [\%] SILAC6 } \\
\text { Ratio } \mathrm{M} / \mathrm{L} \text { count SILAC4 } \\
\text { Ratio H/M count SILAC4 } \\
\text { Ratio } \mathrm{M} / \mathrm{L} \text { count SILAC5 } \\
\text { Ratio H/L count SILAC5 } \\
\text { Ratio M/L count SILAC6 } \\
\text { Ratio H/L count SILAC6 }\end{array}$ & $\begin{array}{l}\text { Selection of columns required for } \\
\text { identification of Imp13 export } \\
\text { candidates }\end{array}$ \\
\hline Matrix 12 & $\begin{array}{l}\text { Categorical } \\
\text { annotation row }\end{array}$ & $\begin{array}{l}\text { Action: Create } \\
\text { Row name: Imp13+Ran/Imp13 } \\
\text { Ratio H/M normalized SILAC4_1 } \\
\text { Ratio M/L normalized SILAC5_1 } \\
\text { Ratio M/L normalized SILAC6_1 }\end{array}$ & $\begin{array}{l}\text { The following ratio group was } \\
\text { defined: } \\
\text { Imp13+Ran/Imp13 }\end{array}$ \\
\hline Matrix 13 & $\begin{array}{l}\text { Categorical } \\
\text { annotation row }\end{array}$ & $\begin{array}{l}\text { Action: Create } \\
\text { Row name: Imp13+Ran/Imp13+Ubc9 } \\
\text { Ratio M/L normalized SILAC4 } \\
\text { Ratio H/L normalized SILAC5_1 } \\
\text { Ratio H/L normalized SILAC6_1 }\end{array}$ & $\begin{array}{l}\text { The following ratio group was } \\
\text { defined: } \\
\text { Imp13+Ran/Imp13+Ubc9 }\end{array}$ \\
\hline Matrix 14 & $\begin{array}{l}\text { Filter rows based on } \\
\text { valid values }\end{array}$ & $\begin{array}{l}\text { Min. number of values: } 3 \\
\text { Mode: In each group } \\
\text { Grouping: Imp13+Ran/Imp13 } \\
\text { Values should be: Greater or equal } \\
\text { Minimum: } 0.5 \\
\text { Filter mode: Add categorical row }\end{array}$ & $\begin{array}{l}\text { Imp13+Ran/Imp13 ratios were filtered } \\
\text { to be } \geq 0.5 \text { for all three replicates. }\end{array}$ \\
\hline Matrix 15 & $\begin{array}{l}\text { Filter rows based on } \\
\text { valid values }\end{array}$ & $\begin{array}{l}\text { Min. number of values: } 3 \\
\text { Mode: In each group } \\
\text { Grouping: Imp13+Ran/Imp13 } \\
\text { Values should be: Greater or equal } \\
\text { Minimum: } 1.5 \\
\text { Filter mode: Add categorical row }\end{array}$ & $\begin{array}{l}\text { Imp13+Ran/Imp13 ratios were filtered } \\
\text { to be } \geq 1.5 \text { for all three replicates. }\end{array}$ \\
\hline Matrix 16 & $\begin{array}{l}\text { Filter rows based on } \\
\text { valid values }\end{array}$ & $\begin{array}{l}\text { Min. number of values: } 3 \\
\text { Mode: In each group } \\
\text { Grouping: Imp13+Ran/Imp13 } \\
\text { Values should be: Greater or equal } \\
\text { Minimum: } 3.0 \\
\text { Filter mode: Add categorical row }\end{array}$ & $\begin{array}{l}\text { Imp13+Ran/Imp13 ratios were filtered } \\
\text { to be } \geq 3.0 \text { for all three replicates. }\end{array}$ \\
\hline Matrix 17 & One-sample test & $\begin{array}{l}\text { Columns: } \\
\text { Ratio H/M normalized SILAC4_1 } \\
\text { Ratio M/L normalized SILAC5_1 } \\
\text { Ratio M/L normalized SILAC6_1 } \\
\text { Value: } 0 \\
\text { Test: t-test } \\
\text { Side: Right } \\
\text { Use for truncation: P value } \\
\text { Threshold p-value: } 0.05 \\
\text {-Log10: tick } \\
\text { Suffix: }\end{array}$ & $\begin{array}{l}\text { One sample-test for determining if the } \\
\text { mean Imp13+Ran/Imp13 ratio is } \\
\text { significantly different from } 0 \text { using a } \\
\text { threshold p-value of } 0.05\end{array}$ \\
\hline
\end{tabular}




\begin{tabular}{|c|c|c|c|}
\hline Matrix & Processing & Settings & Description \\
\hline Matrix 18 & One-sample test & $\begin{array}{l}\text { Columns: } \\
\text { Ratio H/M normalized SILAC4_1 } \\
\text { Ratio M/L normalized SILAC5_1 } \\
\text { Ratio M/L normalized SILAC6_1 } \\
\text { Value: } 0 \\
\text { Test: t-test } \\
\text { Side: Right } \\
\text { Use for truncation: P value } \\
\text { Threshold p-value: } 0.01 \\
\text {-Log10: tick } \\
\text { Suffix: }\end{array}$ & $\begin{array}{l}\text { One sample-test for determining if the } \\
\text { mean Imp13+Ran/Imp13 ratio is } \\
\text { significantly different from } 0 \text { using a } \\
\text { threshold } p \text {-value of } 0.01\end{array}$ \\
\hline Matrix 19 & Rename columns & $\begin{array}{l}\text { Filter } \rightarrow \mathrm{T}(\log 2)=0.5 \\
\text { Filter_ } \rightarrow \mathrm{T}(\log 2)=1.5 \\
\text { Filter_ } \rightarrow \mathrm{T}(\log 2)=3.0 \\
\text { t-test Significant } \rightarrow \text { t-test } 0.05 \\
\text { t-test Significant_ } \rightarrow \text { t-test } 0.01\end{array}$ & $\begin{array}{l}\text { Give expression columns (filtering + } \\
\text { t-test) a more explicit name }\end{array}$ \\
\hline Matrix 20 & $\begin{array}{l}\text { Fill categorical } \\
\text { columns }\end{array}$ & $\begin{array}{l}\text { Columns: } \\
\text { Filter } \rightarrow \mathrm{T}(\log 2)=0.5 \\
\text { Filter_ } \rightarrow \mathrm{T}(\log 2)=1.5 \\
\text { Filter_ } \rightarrow \mathrm{T}(\log 2)=3.0 \\
\text { t-test Significant } \rightarrow \text { t-test } 0.05 \\
\text { t-test Significant_ } \rightarrow \text { t-test } 0.01 \\
\text { Value: - }\end{array}$ & $\begin{array}{l}\text { Fill empty field in categorical columns } \\
\text { with '-' to allow for later combining of } \\
\text { categorical columns }\end{array}$ \\
\hline Matrix 21 & $\begin{array}{l}\text { Combine } \\
\text { categorical columns }\end{array}$ & $\begin{array}{l}\text { First column: t-test } 0.05 \\
\text { Second column: t-test } 0.01\end{array}$ & $\begin{array}{l}\text { Combine categorical columns that } \\
\text { were t-test significant for a threshold } \\
\text { p-value of } 0.05 \text { and } 0.01\end{array}$ \\
\hline Matrix 22 & $\begin{array}{l}\text { Combine } \\
\text { categorical columns }\end{array}$ & $\begin{array}{l}\text { First column: } T(\log 2)=0.5 \\
\text { Second column: } T(\log 2)=1.5\end{array}$ & $\begin{array}{l}\text { Combine categorical columns filtered } \\
\text { for ratio thresholds of } \geq 0.5 \text { and } \geq 1.5\end{array}$ \\
\hline Matrix 23 & $\begin{array}{l}\text { Combine } \\
\text { categorical columns }\end{array}$ & $\begin{array}{l}\text { First column: } T(\log 2)=0.5 \_T(\log 2)=1.5 \\
\text { Second column: } T(\log 2)=3\end{array}$ & $\begin{array}{l}\text { Combine categorical columns filtered } \\
\text { for ratio thresholds of } \geq 0.5, \geq 1.5 \text { and } \\
\geq 3\end{array}$ \\
\hline Matrix 24 & $\begin{array}{l}\text { Combine } \\
\text { categorical columns }\end{array}$ & $\begin{array}{l}\text { First column: } \\
\mathrm{T}(\log 2)=0.5 \_\mathrm{T}(\log 2)=1.5 \_\mathrm{T}(\log 2)=3 \\
\text { Second column: } t \text {-test } 0.05 \_\mathrm{t} \text {-test } \\
0.01\end{array}$ & $\begin{array}{l}\text { Combine categorical columns filtered } \\
\text { for ratio threshold of } \geq 0.5, \geq 1.5 \text { and } \geq \\
3 \text { with t-test significant categorical } \\
\text { column with a threshold p-value of } \\
0.05 \text { and } 0.01 \text {. Required for data } \\
\text { visualization }\end{array}$ \\
\hline Matrix 25 & Average groups & $\begin{array}{l}\text { Grouping: Group1 } \\
\text { Average type: mean } \\
\text { Min. valid values per group: } 1 \\
\text { Keep original data: tick } \\
\text { Add standard deviation: tick }\end{array}$ & $\begin{array}{l}\text { Average Imp13+Ran/Imp13 and } \\
\text { Imp13+Ran/Imp13+Ubc9 ratios from } \\
\text { all three replicates } \\
\text { (Note: averaging is done for all ratios) }\end{array}$ \\
\hline Matrix 26 & $\begin{array}{l}\text { Categorical } \\
\text { annotation row }\end{array}$ & $\begin{array}{l}\text { Action: Create } \\
\text { Row name: Imp13+Ran/Imp13_fwd } \\
\text { Ratio H/M normalized SILAC4_1 }\end{array}$ & $\begin{array}{l}\text { Distinguish between label-switch } \\
\text { experiments for Imp13+Ran/Imp13 } \\
\text { Ratios ('forward reaction') }\end{array}$ \\
\hline Matrix 27 & $\begin{array}{l}\text { Categorical } \\
\text { annotation row }\end{array}$ & $\begin{array}{l}\text { Action: Create } \\
\text { Row name: Imp13+Ran/Imp13_rev } \\
\text { Ratio M/L normalized SILAC5_1 } \\
\text { Ratio M/L normalized SILAC6_1 }\end{array}$ & $\begin{array}{l}\text { Distinguish between label-switch } \\
\text { experiments for Imp13+Ran/Imp13 } \\
\text { Ratios ('reverse reaction') }\end{array}$ \\
\hline Matrix 28 & $\begin{array}{l}\text { Categorical } \\
\text { annotation row }\end{array}$ & $\begin{array}{l}\text { Action: Create } \\
\text { Row name: } \\
\text { Imp13+Ran/Imp13+Ubc9_fwd } \\
\text { Ratio M/L normalized SILAC4 }\end{array}$ & $\begin{array}{l}\text { Distinguish between label-switch } \\
\text { experiments for } \\
\text { Imp13+Ran/Imp13+Ubc9 ratios } \\
\text { ('forward reaction') }\end{array}$ \\
\hline Matrix 29 & $\begin{array}{l}\text { Categorical } \\
\text { annotation row }\end{array}$ & $\begin{array}{l}\text { Action: Create } \\
\text { Row name: } \\
\text { Imp13+Ran/Imp13+Ubc9_rev } \\
\text { Ratio H/L normalized SILAC5_1 } \\
\text { Ratio H/L normalized SILAC6_1 }\end{array}$ & $\begin{array}{l}\text { Distinguish between label-switch } \\
\text { experiments for } \\
\text { Imp13+Ran/Imp13+Ubc9 ratios } \\
\text { ('reverse reaction') }\end{array}$ \\
\hline
\end{tabular}


Appendix

\begin{tabular}{|c|c|c|c|}
\hline Matrix & Processing & Settings & Description \\
\hline Matrix 30 & Average groups & $\begin{array}{l}\text { Grouping: Imp13+Ran/Imp13_fwd } \\
\text { Average type: mean } \\
\text { Min. valid values per group: } 1 \\
\text { Keep original data: tick } \\
\text { Add standard deviation: tick }\end{array}$ & $\begin{array}{l}\text { Average Imp13+Ran/Imp13 ratios } \\
\text { from forward label-switch experiment }\end{array}$ \\
\hline Matrix 31 & Average groups & $\begin{array}{l}\text { Grouping: Imp13+Ran/Imp13_rev } \\
\text { Average type: mean } \\
\text { Min. valid values per group: } 1 \\
\text { Keep original data: tick } \\
\text { Add standard deviation: tick }\end{array}$ & $\begin{array}{l}\text { Average Imp13+Ran/Imp13 ratios } \\
\text { from reverse label-switch experiment }\end{array}$ \\
\hline Matrix 32 & Average groups & $\begin{array}{l}\text { Grouping: } \\
\text { Imp13+Ran/Imp13+Ubc9_fwd } \\
\text { Average type: mean } \\
\text { Min. valid values per group: } 1 \\
\text { Keep original data: tick } \\
\text { Add standard deviation: tick }\end{array}$ & $\begin{array}{l}\text { Average Imp13+Ran/Imp13+Ubc9 } \\
\text { ratios from forward label-switch } \\
\text { experiment }\end{array}$ \\
\hline Matrix 33 & Average groups & $\begin{array}{l}\text { Grouping: } \\
\text { Imp13+Ran/Imp13+Ubc9_rev } \\
\text { Average type: mean } \\
\text { Min. valid values per group: } 1 \\
\text { Keep original data: tick } \\
\text { Add standard deviation: tick }\end{array}$ & $\begin{array}{l}\text { Average Imp13+Ran/Imp13+Ubc9 } \\
\text { ratios from reverse label-switch } \\
\text { experiment }\end{array}$ \\
\hline Matrix 34 & $\begin{array}{l}\text { Filter rows based on } \\
\text { valid values }\end{array}$ & $\begin{array}{l}\text { Min. number of valid values: } 3 \\
\text { Mode: In each group } \\
\text { Grouping: Group } 1 \\
\text { Values should be: Valid } \\
\text { Filter mode: Reduce matrix }\end{array}$ & $\begin{array}{l}\text { To assign annotations all values need } \\
\text { to be valid }\end{array}$ \\
\hline Matrix 35 & Add annotation & $\begin{array}{l}\text { Source: MainPerseusAnnot.txt.gz } \\
\text { UniProt column: Majority protein IDs } \\
\text { Annotations to be added: } \\
\text { GOBP name } \\
\text { GOMF name } \\
\text { GOCC name } \\
\text { KEGG name } \\
\text { Additional sources: }\end{array}$ & $\begin{array}{l}\text { Annotation of: } \\
\text { GOBP: Gene Ontology Biological } \\
\text { Process } \\
\text { GOMF: Gene Ontology Molecular } \\
\text { Function } \\
\text { GOCC: Gene Ontology Cellular } \\
\text { Component } \\
\text { KEGG: Kyoto Encyclopedia of Genes } \\
\text { and Genomes }\end{array}$ \\
\hline
\end{tabular}

Table S5: Perseus workflow for the identification of importin 13 import cargoes (see section 3.3.3 for SILAC experiment)

\begin{tabular}{|l|l|l|l|}
\hline Matrix & Processing & Settings & Description \\
\hline $\begin{array}{l}\text { Matrix } \\
1-10\end{array}$ & Processing steps Matrix 1-10 are identical to Matrix 1-10 in Table S4 \\
\hline
\end{tabular}




\begin{tabular}{|c|c|c|c|}
\hline Matrix & Processing & Settings & Description \\
\hline Matrix 11 & $\begin{array}{l}\text { Reorder/remove } \\
\text { columns }\end{array}$ & $\begin{array}{l}\text { Expression column: } \\
\text { Ratio H/M normalized SILAC4 } \\
\text { Ratio M/L normalized SILAC5 } \\
\text { Ratio M/L normalized SILAC6 } \\
\text { Ratio H/L normalized SILAC4 } \\
\text { Ratio H/M normalized SILAC5_1 } \\
\text { Ratio H/M normalized SILAC6_1 } \\
\text { Numerical columns: } \\
\text { Ratio H/L variability [\%] SILAC4 } \\
\text { Ratio H/M variability [\%] SILAC4 } \\
\text { Ratio M/L variability [\%] SILAC5 } \\
\text { Ratio H/M variability [\%] SILAC5 } \\
\text { Ratio M/L variability [\%] SILAC6 } \\
\text { Ratio H/M variability [\%] SILAC6 } \\
\text { Ratio H/L count SILAC4 } \\
\text { Ratio H/M count SILAC4 } \\
\text { Ratio M/L count SILAC5 } \\
\text { Ratio H/M count SILAC5 } \\
\text { Ratio M/L count SILAC6 } \\
\text { Ratio H/M count SILAC6 }\end{array}$ & $\begin{array}{l}\text { Selection of columns required for } \\
\text { identification of Imp13 import } \\
\text { candidates }\end{array}$ \\
\hline Matrix 12 & $\begin{array}{l}\text { Categorical } \\
\text { annotation row }\end{array}$ & $\begin{array}{l}\text { Action: Create } \\
\text { Row name: Imp13/Imp13+Ran } \\
\text { Ratio H/M normalized SILAC4 } \\
\text { Ratio M/L normalized SILAC5 } \\
\text { Ratio M/L normalized SILAC6 }\end{array}$ & $\begin{array}{l}\text { The following ratio group was } \\
\text { defined: } \\
\text { Imp13/Imp13+Ran }\end{array}$ \\
\hline Matrix 13 & $\begin{array}{l}\text { Categorical } \\
\text { annotation row }\end{array}$ & $\begin{array}{l}\text { Action: Create } \\
\text { Row name: Imp13/Imp13+Ubc9 } \\
\text { Ratio H/L normalized SILAC4 } \\
\text { Ratio H/M normalized SILAC5_1 } \\
\text { Ratio H/M normalized SILAC6_1 }\end{array}$ & $\begin{array}{l}\text { The following ratio group was } \\
\text { defined: } \\
\text { Imp13/Imp13+Ubc9 }\end{array}$ \\
\hline Matrix 14 & $\begin{array}{l}\text { Filter rows based on } \\
\text { valid values }\end{array}$ & $\begin{array}{l}\text { Min. number of values: } 3 \\
\text { Mode: In each group } \\
\text { Grouping: Group1 } \\
\text { Values should be: Greater or equal } \\
\text { Minimum: } 0.5 \\
\text { Filter mode: Add categorical row }\end{array}$ & $\begin{array}{l}\text { Imp13/Imp13+Ran \& } \\
\text { Imp13/Imp13+Ubc9 ratios were } \\
\text { filtered to be } \geq 0.5 \text { for all three } \\
\text { replicates. }\end{array}$ \\
\hline Matrix 15 & $\begin{array}{l}\text { Filter rows based on } \\
\text { valid values }\end{array}$ & $\begin{array}{l}\text { Min. number of values: } 3 \\
\text { Mode: In each group } \\
\text { Grouping: Group1 } \\
\text { Values should be: Greater or equal } \\
\text { Minimum: } 1.5 \\
\text { Filter mode: Add categorical row }\end{array}$ & $\begin{array}{l}\text { Imp13/Imp13+Ran \& } \\
\text { Imp13/Imp13+Ubc9 ratios were } \\
\text { filtered to be } \geq 1.5 \text { for all three } \\
\text { replicates. }\end{array}$ \\
\hline Matrix 16 & $\begin{array}{l}\text { Filter rows based on } \\
\text { valid values }\end{array}$ & $\begin{array}{l}\text { Min. number of values: } 3 \\
\text { Mode: In each group } \\
\text { Grouping: Group1 } \\
\text { Values should be: Greater or equal } \\
\text { Minimum: } 3.0 \\
\text { Filter mode: Add categorical row }\end{array}$ & $\begin{array}{l}\text { Imp13/Imp13+Ran \& } \\
\text { Imp13/Imp13+Ubc9 ratios were } \\
\text { filtered to be } \geq 3.0 \text { for all three } \\
\text { replicates. }\end{array}$ \\
\hline Matrix 17 & One-sample test & $\begin{array}{l}\text { Columns: } \\
\text { Ratio H/M normalized SILAC4 } \\
\text { Ratio M/L normalized SILAC5 } \\
\text { Ratio M/L normalized SILAC6 } \\
\text { Ratio H/L normalized SILAC4 } \\
\text { Ratio H/M normalized SILAC5_1 } \\
\text { Ratio H/M normalized SILAC6_1 } \\
\text { Value: } 0 \\
\text { Test: t-test } \\
\text { Side: Right } \\
\text { Use for truncation: P value } \\
\text { Threshold p-value: } 0.05 \\
\text {-Log10: tick } \\
\text { Suffix: }\end{array}$ & $\begin{array}{l}\text { One sample-test for determining if the } \\
\text { mean Imp13/Imp13+Ran \& } \\
\text { Imp13/Imp13+Ubc9 ratios are } \\
\text { significantly different from } 0 \text { using a } \\
\text { threshold p-value of } 0.05\end{array}$ \\
\hline
\end{tabular}




\begin{tabular}{|c|c|c|c|}
\hline Matrix & Processing & Settings & Description \\
\hline Matrix 18 & One-sample test & $\begin{array}{l}\text { Columns: } \\
\text { Ratio H/M normalized SILAC4 } \\
\text { Ratio M/L normalized SILAC5 } \\
\text { Ratio M/L normalized SILAC6 } \\
\text { Ratio H/L normalized SILAC4 } \\
\text { Ratio H/M normalized SILAC5_1 } \\
\text { Ratio H/M normalized SILAC6_1 } \\
\text { Value: } 0 \\
\text { Test: t-test } \\
\text { Side: Right } \\
\text { Use for truncation: P value } \\
\text { Threshold p-value: } 0.01 \\
\text {-Log10: tick } \\
\text { Suffix: }\end{array}$ & $\begin{array}{l}\text { One sample-test for determining if the } \\
\text { mean Imp13/Imp13+Ran \& } \\
\text { Imp13/Imp13+Ubc9 ratios are } \\
\text { significantly different from } 0 \text { using a } \\
\text { threshold p-value of } 0.01\end{array}$ \\
\hline Matrix 19 & Rename columns & $\begin{array}{l}\text { Filter } \rightarrow \mathrm{T}(\log 2)=0.5 \\
\text { Filter_ } \rightarrow \mathrm{T}(\log 2)=1.5 \\
\text { Filter_ } \rightarrow \mathrm{T}(\log 2)=3.0 \\
\text { t-test Significant } \rightarrow \text { t-test } 0.05 \\
\text { t-test Significant_ } \rightarrow \text { t-test } 0.01\end{array}$ & $\begin{array}{l}\text { Give expression columns (filtering + } \\
\text { t-test) a more explicit name }\end{array}$ \\
\hline Matrix 20 & $\begin{array}{l}\text { Fill categorical } \\
\text { columns }\end{array}$ & $\begin{array}{l}\text { Columns: } \\
\text { Filter } \rightarrow \mathrm{T}(\log 2)=0.5 \\
\text { Filter_ } \rightarrow \mathrm{T}(\log 2)=1.5 \\
\text { Filter_ } \rightarrow \mathrm{T}(\log 2)=3.0 \\
\text { t-test Significant } \rightarrow \text { t-test } 0.05 \\
\text { t-test Significant_ } \rightarrow \text { t-test } 0.01 \\
\text { Value: - }\end{array}$ & $\begin{array}{l}\text { Fill empty field in categorical columns } \\
\text { with '-' to allow for later combining of } \\
\text { categorical columns }\end{array}$ \\
\hline Matrix 21 & $\begin{array}{l}\text { Combine } \\
\text { categorical columns }\end{array}$ & $\begin{array}{l}\text { First column: t-test } 0.05 \\
\text { Second column: t-test } 0.01\end{array}$ & $\begin{array}{l}\text { Combine categorical columns that } \\
\text { were t-test significant for a threshold } \\
\text { p-value of } 0.05 \text { and } 0.01\end{array}$ \\
\hline Matrix 22 & $\begin{array}{l}\text { Combine } \\
\text { categorical columns }\end{array}$ & $\begin{array}{l}\text { First column: } T(\log 2)=0.5 \\
\text { Second column: } T(\log 2)=1.5\end{array}$ & $\begin{array}{l}\text { Combine categorical columns filtered } \\
\text { for ratio thresholds of } \geq 0.5 \text { and } \geq 1.5\end{array}$ \\
\hline Matrix 23 & $\begin{array}{l}\text { Combine } \\
\text { categorical columns }\end{array}$ & $\begin{array}{l}\text { First column: } T(\log 2)=0.5-T(\log 2)=1.5 \\
\text { Second column: } T(\log 2)=3\end{array}$ & $\begin{array}{l}\text { Combine categorical columns filtered } \\
\text { for ratio thresholds of } \geq 0.5, \geq 1.5 \text { and } \\
\geq 3\end{array}$ \\
\hline Matrix 24 & $\begin{array}{l}\text { Combine } \\
\text { categorical columns }\end{array}$ & $\begin{array}{l}\text { First column: } \\
\mathrm{T}(\log 2)=0.5 \_\mathrm{T}(\log 2)=1.5 \_\mathrm{T}(\log 2)=3 \\
\text { Second column: } \mathrm{t} \text {-test } 0.05 \_ \text {t-test } \\
0.01\end{array}$ & $\begin{array}{l}\text { Combine categorical columns filtered } \\
\text { for ratio threshold of } \geq 0.5, \geq 1.5 \text { and } \geq \\
3 \text { with t-test significant categorical } \\
\text { column with a threshold p-value of } \\
0.05 \text { and } 0.01 \text {. Required for data } \\
\text { visualization }\end{array}$ \\
\hline Matrix 25 & Average groups & $\begin{array}{l}\text { Grouping: Group1 } \\
\text { Average type: mean } \\
\text { Min. valid values per group: } 1 \\
\text { Keep original data: tick } \\
\text { Add standard deviation: tick }\end{array}$ & $\begin{array}{l}\text { Average Imp13/Imp13+Ran and } \\
\text { Imp13/Imp13+Ubc9 ratios from all } \\
\text { three replicates }\end{array}$ \\
\hline Matrix 26 & $\begin{array}{l}\text { Categorical } \\
\text { annotation row }\end{array}$ & $\begin{array}{l}\text { Action: Create } \\
\text { Row name: Imp13/Imp13+Ran_fwd } \\
\text { Ratio H/M normalized SILAC4 }\end{array}$ & $\begin{array}{l}\text { Distinguish between label-switch } \\
\text { experiments for Imp13/Imp13+Ran } \\
\text { Ratios ('forward reaction') }\end{array}$ \\
\hline Matrix 27 & $\begin{array}{l}\text { Categorical } \\
\text { annotation row }\end{array}$ & $\begin{array}{l}\text { Action: Create } \\
\text { Row name: Imp13/Imp13+Ran_rev } \\
\text { Ratio M/L normalized SILAC5 } \\
\text { Ratio M/L normalized SILAC6 }\end{array}$ & $\begin{array}{l}\text { Distinguish between label-switch } \\
\text { experiments for Imp13/Imp13+Ran } \\
\text { Ratios ('reverse reaction') }\end{array}$ \\
\hline Matrix 28 & $\begin{array}{l}\text { Categorical } \\
\text { annotation row }\end{array}$ & $\begin{array}{l}\text { Action: Create } \\
\text { Row name: Imp13/Imp13+Ubc9_fwd } \\
\text { Ratio H/L normalized SILAC4 }\end{array}$ & $\begin{array}{l}\text { Distinguish between label-switch } \\
\text { experiments for } \\
\text { Imp13/Imp13+Ubc9 ratios ('forward } \\
\text { reaction') }\end{array}$ \\
\hline Matrix 29 & $\begin{array}{l}\text { Categorical } \\
\text { annotation row }\end{array}$ & $\begin{array}{l}\text { Action: Create } \\
\text { Row name: Imp13/Imp13+Ubc9_rev } \\
\text { Ratio H/M normalized SILAC5_1 } \\
\text { Ratio H/M normalized SILAC6_1 }\end{array}$ & $\begin{array}{l}\text { Distinguish between label-switch } \\
\text { experiments for } \\
\text { Imp13/Imp13+Ubc9 ratios ('reverse } \\
\text { reaction') }\end{array}$ \\
\hline
\end{tabular}


Appendix

\begin{tabular}{|c|c|c|c|}
\hline Matrix & Processing & Settings & Description \\
\hline Matrix 30 & Average groups & $\begin{array}{l}\text { Grouping: Imp13/Imp13+Ran_fwd } \\
\text { Average type: mean } \\
\text { Min. valid values per group: } 1 \\
\text { Keep original data: tick } \\
\text { Add standard deviation: tick }\end{array}$ & $\begin{array}{l}\text { Average Imp13/Imp13+Ran ratios } \\
\text { from forward label-switch experiment }\end{array}$ \\
\hline Matrix 31 & Average groups & $\begin{array}{l}\text { Grouping: Imp13/Imp13+Ran_rev } \\
\text { Average type: mean } \\
\text { Min. valid values per group: } 1 \\
\text { Keep original data: tick } \\
\text { Add standard deviation: tick }\end{array}$ & $\begin{array}{l}\text { Average Imp13/Imp13+Ran ratios } \\
\text { from reverse label-switch experiment }\end{array}$ \\
\hline Matrix 32 & Average groups & $\begin{array}{l}\text { Grouping: Imp13/Imp13+Ubc9_fwd } \\
\text { Average type: mean } \\
\text { Min. valid values per group: } 1 \\
\text { Keep original data: tick } \\
\text { Add standard deviation: tick }\end{array}$ & $\begin{array}{l}\text { Average Imp13/Imp13+Ubc9 ratios } \\
\text { from forward label-switch experiment }\end{array}$ \\
\hline Matrix 33 & Average groups & $\begin{array}{l}\text { Grouping: Imp13/Imp13+Ubc9_rev } \\
\text { Average type: mean } \\
\text { Min. valid values per group: } 1 \\
\text { Keep original data: tick } \\
\text { Add standard deviation: tick }\end{array}$ & $\begin{array}{l}\text { Average Imp13/Imp13+Ubc9 ratios } \\
\text { from reverse label-switch experiment }\end{array}$ \\
\hline Matrix 34 & $\begin{array}{l}\text { Filter rows based on } \\
\text { valid values }\end{array}$ & $\begin{array}{l}\text { Min. number of valid values: } 3 \\
\text { Mode: In each group } \\
\text { Grouping: Group } 1 \\
\text { Values should be: Valid } \\
\text { Filter mode: Reduce matrix }\end{array}$ & $\begin{array}{l}\text { To assign annotations all values need } \\
\text { to be valid }\end{array}$ \\
\hline Matrix 35 & Add annotation & $\begin{array}{l}\text { Source: MainPerseusAnnot.txt.gz } \\
\text { UniProt column: Majority protein IDs } \\
\text { Annotations to be added: } \\
\text { GOBP name } \\
\text { GOMF name } \\
\text { GOCC name } \\
\text { KEGG name } \\
\text { Additional sources: }\end{array}$ & $\begin{array}{l}\text { Annotation of: } \\
\text { GOBP: Gene Ontology Biological } \\
\text { Process } \\
\text { GOMF: Gene Ontology Molecular } \\
\text { Function } \\
\text { GOCC: Gene Ontology Cellular } \\
\text { Component } \\
\text { KEGG: Kyoto Encyclopedia of Genes } \\
\text { and Genomes }\end{array}$ \\
\hline
\end{tabular}

Table S6: List of potential importin 13 import cargoes identified in SILAC screen (log2 SILAC ratio $>0.5$ for all three experimental replicates, see section 3.3.3 for experimental details) ${ }^{\#}$

\begin{tabular}{|c|c|c|c|c|c|}
\hline $\begin{array}{l}\text { Uniprot } \\
\text { ID }\end{array}$ & Protein Name & Gene & $\begin{array}{l}\text { log2(Imp13I } \\
\text { Imp13+Ran) }\end{array}$ & $\begin{array}{l}\log 2(\operatorname{Imp} 13 / \\
\text { Imp13+Ubc9) }\end{array}$ & $\begin{array}{l}-\log \\
\text { (p value)* }^{*}\end{array}$ \\
\hline \multicolumn{6}{|c|}{ Proteins identified for both Imp13/Imp13+Ran \& Imp13/Imp13+Ubc9 } \\
\hline Q92538 & Golgi-specific brefeldin A-resistance guanine nucleotide exchange factor 1 & GBF1 & 0.813033 & 4.71873 & 1.86233 \\
\hline Q92888 & Rho guanine nucleotide exchange factor 1 & ARHGEF1 & 1.42738 & 3.78382 & 2.63594 \\
\hline Q6PGP7 & Tetratricopeptide repeat protein 37 & TTC37 & 0.939691 & 3.65913 & 2.11507 \\
\hline Q14247 & Src substrate cortactin & CTTN & 1.75313 & 3.62126 & 3.11061 \\
\hline O75815 & Breast cancer anti-estrogen resistance protein 3 & BCAR3 & 1.26153 & 3.23052 & 2.69767 \\
\hline Q12800 & Alpha-globin transcription factor $\mathrm{CP} 2$ & TFCP2 & 1.06516 & 2.75921 & 2.5538 \\
\hline Q14C86 & GTPase-activating protein and VPS9 domain-containing protein 1 & GAPVD1 & 1.4609 & 2.68049 & 3.40625 \\
\hline Q12774 & Rho guanine nucleotide exchange factor 5 & ARHGEF5 & 0.705027 & 2.41263 & 2.30914 \\
\hline Q15025 & TNFAIP3-interacting protein 1 & TNIP1 & 0.95981 & 2.24084 & 2.85848 \\
\hline O43896 & Kinesin-like protein KIF1C & KIF1C & 1.11685 & 2.10933 & 3.33456 \\
\hline Q13263 & Transcription intermediary factor 1-beta & TRIM28 & 0.867331 & 1.90373 & 2.95866 \\
\hline Q3V6T2 & Girdin & CCDC88A & 0.981046 & 1.86711 & 2.40064 \\
\hline P23258 & Tubulin gamma-1 chain & TUBG1 & 0.693187 & 1.63586 & 2.83976 \\
\hline Q8IV48 & 3-5 exoribonuclease 1 & ERI1 & 0.93069 & 1.46739 & 3.46771 \\
\hline O15084 & Serine/threonine-protein phosphatase 6 regulatory ankyrin repeat subunit $A$ & ANKRD28 & 1.04204 & 0.864303 & 4.21767 \\
\hline Q92974 & Rho guanine nucleotide exchange factor 2 & ARHGEF2 & 1.0197 & 0.829202 & 4.22529 \\
\hline \multicolumn{6}{|c|}{ Proteins identified for Imp13/Imp13+Ran } \\
\hline Q9BPX7 & UPF0415 protein C7orf25 & C7orf25 & 3.80688 & 0.642 & 3.00728 \\
\hline
\end{tabular}




\begin{tabular}{|c|c|c|c|c|c|}
\hline $\begin{array}{l}\text { Uniprot } \\
\text { ID }\end{array}$ & Protein Name & Gene & $\begin{array}{l}\text { Iog2(Imp13/ } \\
\text { Imp13+Ran) }\end{array}$ & $\begin{array}{l}\text { log2(Imp13I } \\
\text { Imp13+Ubc9) }\end{array}$ & $\begin{array}{l}-\log \\
(p \text { value })^{*}\end{array}$ \\
\hline 000303 & Eukaryotic translation initiation factor 3 subunit $F$ & EIF3F & 1.71919 & -0.0768488 & 1.84627 \\
\hline 075821 & Eukaryotic translation initiation factor 3 subunit G & EIF3G & 1.63573 & 0.0269878 & 1.74379 \\
\hline Q14152 & Eukaryotic translation initiation factor 3 subunit $A$ & EIF3A & 1.62644 & -0.137368 & 1.75614 \\
\hline 015479 & Melanoma-associated antigen B2 & MAGEB2 & 1.53793 & -0.195129 & 1.57917 \\
\hline Q7L2H7 & Eukaryotic translation initiation factor 3 subunit $\mathrm{M}$ & EIF3M & 1.50969 & -0.00783382 & 1.74761 \\
\hline P05783 & Keratin, type I cytoskeletal 18 & KRT18 & 1.4847 & 2.92434 & 1.59389 \\
\hline P55884 & Eukaryotic translation initiation factor 3 subunit B & EIF3B & 1.47532 & -0.140885 & 1.88281 \\
\hline P36578 & $60 S$ ribosomal protein $\mathrm{L} 4$ & RPL4 & 1.41764 & -0.229922 & 1.52031 \\
\hline Q9NSD9 & Phenylalanine--tRNA ligase beta subunit & FARSB & 1.41105 & -0.0168935 & 1.46055 \\
\hline Q53EL6 & $\underline{\text { Programmed cell death protein }}^{* *}$ & PDCD4 & 1.38179 & -0.239508 & 1.9684 \\
\hline Q9P0J7 & E3 ubiquitin-protein ligase KCMF1 & KCMF1 & 1.38113 & -0.366198 & 2.02826 \\
\hline P60228 & Eukaryotic translation initiation factor 3 subunit $\mathrm{E}$ & EIF3E & 1.37905 & 0.0827138 & 1.74038 \\
\hline С9J9K3 & $40 \mathrm{~S}$ ribosomal protein SA & RPSA & 1.35307 & -0.143419 & 1.56352 \\
\hline Q99613 & Eukaryotic translation initiation factor 3 subunit $C$ & EIF3C & 1.32125 & -0.0816751 & 1.70334 \\
\hline P39023 & $60 \mathrm{~S}$ ribosomal protein L3 & RPL3 & 1.30222 & -0.244874 & 1.51126 \\
\hline Q9Y262 & Eukaryotic translation initiation factor 3 subunit $\mathrm{L}$ & EIF3L & 1.28118 & -0.0190674 & 1.77652 \\
\hline Q13347 & Eukaryotic translation initiation factor 3 subunit I & EIF3I & 1.20545 & 0.0620854 & 1.38953 \\
\hline Q5T4S7 & E3 ubiquitin-protein ligase UBR4 & UBR4 & 1.12458 & -0.358052 & 1.77364 \\
\hline Q9Y285 & Phenylalanine--tRNA ligase alpha subunit & FARSA & 1.10485 & 0.273047 & 1.68737 \\
\hline Q99615 & DnaJ homolog subfamily $\mathrm{C}$ member 7 & DNAJC7 & 1.05021 & -0.141127 & 2.01229 \\
\hline Q13895 & Bystin & BYSL & 0.812382 & -0.246966 & 2.6437 \\
\hline $\mathrm{H} 7 \mathrm{~B} \times \mathrm{H} 2$ & Serine/threonine-protein phosphatase 6 regulatory subunit 3 & PPP6R3 & 0.63477 & 0.492251 & 2.99464 \\
\hline \multicolumn{6}{|c|}{ Proteins identified for Imp13/Imp13+Ubc9 } \\
\hline Q9NRG0 & Chromatin accessibility complex protein 1 & CHRAC1 & 0.417418 & 5.31296 & 3.99816 \\
\hline Q9NRF9 & DNA polymerase epsilon subunit 3 & POLE3 & 0.168921 & 5.01915 & 2.80557 \\
\hline Q9Y530 & O-acetyl-ADP-ribose deacetylase 1 & OARD1 & -0.00054066 & 4.49774 & 2.52061 \\
\hline Q9Y314 & Nitric oxide synthase-interacting protein & NOSIP & 0.364505 & 4.48227 & 3.23087 \\
\hline Q9NR33 & DNA polymerase epsilon subunit 4 & POLE4 & -0.243075 & 3.91994 & 2.54084 \\
\hline P62380 & TATA box-binding protein-like protein 1 & TBPL1 & -0.318334 & 3.90187 & 2.173 \\
\hline Q16513 & Serine/threonine-protein kinase N2 & PKN2 & -0.2442 & 3.57229 & 4.13079 \\
\hline Q15477 & Helicase SKI2W & SKIV2L & 0.624186 & 3.46386 & 2.80251 \\
\hline Q9GZS3 & WD repeat-containing protein 61 & WDR61 & 0.269134 & 3.43407 & 2.461 \\
\hline P27540 & Aryl hydrocarbon receptor nuclear translocator & ARNT & 0.470754 & 3.28838 & 2.69279 \\
\hline Q96JG6 & Coiled-coil domain-containing protein 132 & CCDC132 & 0.120687 & 3.27985 & 2.09074 \\
\hline Q9NRF2 & SH2B adapter protein 1 & SH2B1 & 0.95146 & 3.15032 & 3.11739 \\
\hline Q5VIR6 & Vacuolar protein sorting-associated protein 53 homolog & VPS53 & 0.229217 & 3.07472 & 2.87329 \\
\hline P23588 & Eukaryotic translation initiation factor 4B & EIF4B & 0.283411 & 3.01306 & 2.62681 \\
\hline Q9Y606 & tRNA pseudouridine synthase $A$, mitochondrial & PUS1 & -0.255249 & 2.97562 & 2.86354 \\
\hline P05783 & Keratin, type I cytoskeletal 18 & KRT18 & 1.4847 & 2.92434 & 2.4275 \\
\hline J3KNCO & Transcription initiation factor IIA subunit 1 & GTF2A1 & -0.232213 & 2.86042 & 2.21091 \\
\hline P52657 & $\underline{\text { Transcription initiation factor IIA subunit } 2^{* *}}$ & GTF2A2 & -0.805258 & 2.83151 & 2.22288 \\
\hline P53999 & Activated RNA polymerase II transcriptional coactivator p15 & SUB1 & -1.50329 & 2.80937 & 2.53168 \\
\hline Q9NQT8 & Kinesin-like protein KIF13B & KIF13B & 0.839283 & 2.64362 & 3.32859 \\
\hline Q8WUF5 & RelA-associated inhibitor & PPP1R13L & 0.646154 & 2.61013 & 2.93803 \\
\hline Q9P1Y5 & Calmodulin-regulated spectrin-associated protein 3 & CAMSAP3 & -0.040654 & 2.58535 & 2.84022 \\
\hline Q9BYX4 & Interferon-induced helicase $\mathrm{C}$ domain-containing protein 1 & $\mathrm{IFIH} 1$ & -0.0877257 & 2.54065 & 2.77674 \\
\hline E3W994 & CLIP-associating protein 2 & CLASP2 & 0.175809 & 2.52605 & 3.97909 \\
\hline 060343 & TBC1 domain family member 4 & TBC1D4 & 0.433131 & 2.4178 & 3.09736 \\
\hline Q9Y4H2 & Insulin receptor substrate 2 & IRS2 & 0.310145 & 2.35194 & 2.34747 \\
\hline Q14157 & Ubiquitin-associated protein 2-like & UBAP2L & 0.4474 & 2.19262 & 1.91864 \\
\hline C9JZR2 & Catenin delta-1 & CTNND1 & -0.535033 & 2.16381 & 2.70911 \\
\hline P13984 & General transcription factor IIF subunit 2 & GTF2F2 & -0.29533 & 2.1155 & 2.8392 \\
\hline Q9UGJ1 & Gamma-tubulin complex component 4 & TUBGCP4 & 0.444484 & 2.07017 & 2.982 \\
\hline P62136 & Serine/threonine-protein phosphatase PP1-alpha catalytic subunit & PPP1CA & -0.0792858 & 2.05476 & 2.28248 \\
\hline Q16204 & Coiled-coil domain-containing protein 6 & CCDC6 & 0.484694 & 2.05259 & 2.49215 \\
\hline P61956 & Small ubiquitin-related modifier & SUMO2,3,4 & 0.131713 & 2.04704 & 1.39415 \\
\hline C9JEL3 & Eukaryotic translation initiation factor 4E type 2 & EIF4E2 & -0.31437 & 1.98722 & 4.11817 \\
\hline Q14693 & Phosphatidate phosphatase LPIN1 & LPIN1 & 0.468605 & 1.96606 & 2.188 \\
\hline P62140 & Serine/threonine-protein phosphatase PP1-beta catalytic subunit & PPP1CB & 0.168797 & 1.95883 & 1.34722 \\
\hline H7C170 & Uncharacterized methyltransferase WBSCR22 & WBSCR22 & 0.484091 & 1.89476 & 2.03673 \\
\hline Q9Y6R0 & Numb-like protein & NUMBL & -0.260254 & 1.87456 & 2.35055 \\
\hline Q7Z460 & CLIP-associating protein 1 & CLASP1 & 0.538826 & 1.86087 & 2.3655 \\
\hline Q8TEW0 & Partitioning defective 3 homolog & PARD3 & -0.15918 & 1.85178 & 2.34429 \\
\hline Q86UU1 & Pleckstrin homology-like domain family B member 1 & PHLDB1 & 0.222936 & 1.80549 & 1.97365 \\
\hline Q8WX93 & Palladin & PALLD & 0.367969 & 1.74021 & 2.26034 \\
\hline Q96F86 & Enhancer of mRNA-decapping protein $3^{* *}$ & EDC3 & 0.867101 & 1.72773 & 2.35559 \\
\hline Q92620 & Pre-mRNA-splicing factor ATP-dependent RNA helicase PRP16 & DHX38 & 0.403327 & 1.71743 & 2.29087 \\
\hline Q3MHD2 & Protein LSM12 homolog & LSM12 & -0.180633 & 1.712 & 2.40183 \\
\hline Q14244 & Ensconsin & MAP7 & -0.300793 & 1.69568 & 1.81708 \\
\hline Q00403 & Transcription initiation factor IIB & GTF2B & -1.80555 & 1.67293 & 1.43181 \\
\hline
\end{tabular}




\begin{tabular}{|c|c|c|c|c|c|}
\hline $\begin{array}{l}\text { Uniprot } \\
\text { ID }\end{array}$ & Protein Name & Gene & $\begin{array}{l}\text { Iog2(Imp13I } \\
\text { Imp13+Ran) }\end{array}$ & $\begin{array}{l}\text { log2(Imp13I } \\
\text { Imp13+Ubc9) }\end{array}$ & $\begin{array}{l}-\log \\
(p \text { value })^{\star}\end{array}$ \\
\hline Q14919 & Dr1-associated corepressor & DRAP1 & -0.434796 & 1.6483 & 2.10521 \\
\hline Q9U130 & tRNA methyltransferase 112 homolog & TRMT112 & -0.31314 & 1.63061 & 1.77601 \\
\hline P41743 & Protein kinase $\mathrm{C}$ iota type & $\mathrm{PRKCl}$ & -0.36483 & 1.62035 & 2.48096 \\
\hline P15924 & Desmoplakin & DSP & 0.408405 & 1.61421 & 1.28908 \\
\hline Q8ND56 & Protein LSM14 homolog A & LSM14A & -0.890691 & 1.6105 & 2.63839 \\
\hline P50548 & ETS domain-containing transcription factor ERF & ERF & -0.875577 & 1.60979 & 2.9159 \\
\hline P49757 & Protein numb homolog & NUMB & -0.429043 & 1.60483 & 2.4028 \\
\hline Q8WWI1 & LIM domain only protein 7 & LMO7 & 0.229279 & 1.59937 & 1.91315 \\
\hline Q8IUD2 & ELKS/Rab6-interacting/CAST family member 1 & ERC1 & 0.257515 & 1.59886 & 2.05536 \\
\hline 075420 & PERQ amino acid-rich with GYF domain-containing protein 1 & GIGYF1 & -0.216063 & 1.59731 & 2.48073 \\
\hline Q8NEY1 & Neuron navigator 1 & NAV1 & -0.465412 & 1.59462 & 1.97797 \\
\hline Q15427 & Splicing factor 3B subunit 4 & SF3B4 & 0.552802 & 1.57273 & 2.55564 \\
\hline Q01658 & Protein Dr1 & DR1 & -0.480059 & 1.54603 & 2.12448 \\
\hline B7Z5N5 & Mothers against decapentaplegic homolog 2 & SMAD2 & -0.961146 & 1.53707 & 2.66277 \\
\hline B1ALK7 & Rho guanine nucleotide exchange factor 7 & ARHGEF7 & 0.906418 & 1.52958 & 2.17687 \\
\hline Q08AD1 & Calmodulin-regulated spectrin-associated protein 2 & CAMSAP2 & -0.240718 & 1.47753 & 2.89932 \\
\hline P47813 & Eukaryotic translation initiation factor $1 \mathrm{~A}$ & EIF1A & -3.25237 & 1.42849 & 2.37898 \\
\hline Q92900 & Regulator of nonsense transcripts 1 & UPF1 & -1.15652 & 1.3723 & 2.60415 \\
\hline B1AKN7 & Nuclear factor 1 A-type & NFIA & 0.627497 & 1.36784 & 2.14935 \\
\hline Q13435 & Splicing factor 3B subunit 2 & SF3B2 & 0.318339 & 1.35689 & 2.11956 \\
\hline P61978 & Heterogeneous nuclear ribonucleoprotein $\mathrm{K}$ & HNRNPK & -1.19892 & 1.3447 & 1.8493 \\
\hline P48729 & Casein kinase I isoform alpha & CSNK1A1 & -1.06365 & 1.31741 & 2.39073 \\
\hline Q04917 & 14-3-3 protein eta & YWHAH & -0.337327 & 1.3135 & 2.25234 \\
\hline P61981 & 14-3-3 protein gamma & YWHAG & -0.28702 & 1.31075 & 2.27926 \\
\hline E7EV99 & Alpha-adducin & ADD1 & -0.922004 & 1.30584 & 1.81035 \\
\hline Q684P5 & Rap1 GTPase-activating protein 2 & RAP1GAP2 & 0.541901 & 1.28222 & 1.71409 \\
\hline Q7Z3U7 & Protein MON2 homolog & MON2 & 0.758447 & 1.27967 & 1.61195 \\
\hline Q13501 & Sequestosome-1 ${ }^{* *}$ & SQSTM1 & 0.755231 & 1.26888 & 2.0583 \\
\hline F5GWN5 & $\begin{array}{l}\text { Phosphatidylinositol 4-phosphate 3-kinase C2 domain-containing subunit } \\
\text { beta }\end{array}$ & PIK3C2B & -0.2512 & 1.26783 & 1.97628 \\
\hline P26196 & Probable ATP-dependent RNA helicase DDX6 & DDX6 & -0.713644 & 1.21929 & 3.03261 \\
\hline Q9BXB4 & Oxysterol-binding protein-related protein 11 & OSBPL11 & 0.366041 & 1.19809 & 2.94503 \\
\hline P31946 & 14-3-3 protein beta/alpha & YWHAB & -0.686851 & 1.16229 & 2.04505 \\
\hline Q9P270 & SLAIN motif-containing protein 2 & SLAIN2 & -1.01011 & 1.12969 & 1.70625 \\
\hline P62258 & 14-3-3 protein epsilon & YWHAE & -0.501352 & 1.11888 & 2.19164 \\
\hline Q96SU4 & Oxysterol-binding protein-related protein 9 & OSBPL9 & 0.370393 & 1.07767 & 2.34754 \\
\hline P78344 & Eukaryotic translation initiation factor 4 gamma 2 & EIF4G2 & 0.29085 & 1.01729 & 2.88712 \\
\hline Q14161 & ARF GTPase-activating protein GIT2 & GIT2 & 0.717892 & 1.00944 & 1.67464 \\
\hline O60825 & 6-phosphofructo-2-kinase/fructose-2,6-bisphosphatase 2 & PFKFB2 & -3.29633 & 1.00394 & 1.84653 \\
\hline P27348 & $14-3-3$ protein theta & YWHAQ & -0.922545 & 0.961431 & 1.82235 \\
\hline P63104 & 14-3-3 protein zeta/delta & YWHAZ & -0.85652 & 0.961046 & 1.96388 \\
\hline Q96HC4 & PDZ and LIM domain protein 5 & PDLIM5 & -2.97543 & 0.955176 & 2.8885 \\
\hline Q9BXB5 & Oxysterol-binding protein-related protein 10 & OSBPL10 & 0.238173 & 0.926031 & 2.18881 \\
\hline P05549 & Transcription factor AP-2-alpha & TFAP2A & -0.638872 & 0.924126 & 2.26235 \\
\hline Q6ZSZ5 & Rho guanine nucleotide exchange factor 18 & ARHGEF18 & -0.150335 & 0.919987 & 1.44517 \\
\hline Q86W92 & Liprin-beta-1 & PPFIBP1 & 0.340308 & 0.889209 & 2.54334 \\
\hline B7Z7P8 & Eukaryotic peptide chain release factor subunit 1 & ETF1 & -1.00667 & 0.872366 & 1.67055 \\
\hline P12956 & X-ray repair cross-complementing protein 6 & XRCC6 & -0.977419 & 0.841513 & 1.51525 \\
\hline P61326 & Protein mago nashi homolog & MAGOH & -0.724602 & 0.814207 & 2.25492 \\
\hline Q7Z2W4 & Zinc finger $\mathrm{CCCH}$-type antiviral protein 1 & ZC3HAV1 & 0.701945 & 0.791835 & 2.22414 \\
\hline 000571 & ATP-dependent RNA helicase DDX3 & DDX3 & 0.664858 & 0.780721 & 1.90872 \\
\hline HOYM23 & Ankyrin repeat domain-containing protein 17 & ANKRD17 & -0.532403 & 0.780196 & 2.383 \\
\hline Q9UHR6 & Zinc finger HIT domain-containing protein 2 & ZNHIT2 & 0.501032 & 0.753856 & 2.71696 \\
\hline P49368 & T-complex protein 1 subunit gamma & ССТ3 & -0.731554 & 0.739372 & 2.11106 \\
\hline P06493 & Cyclin-dependent kinase 1 & CDK1 & -1.34662 & 0.738885 & 2.26621 \\
\hline 000743 & Serine/threonine-protein phosphatase 6 catalytic subunit & PPP6C & 0.261494 & 0.71037 & 2.52078 \\
\hline \multicolumn{6}{|c|}{ Proteins analyzed that were identified for both Imp13/Imp13+Ran \& Imp13/Imp13+Ubc9 for two out of three SILAC experiments } \\
\hline $\mathrm{F} 2 \mathrm{Z} 2 \mathrm{~T} 2$ & DNA repair protein complementing XP-A cells ${ }^{* *}$ & XPA & 0.783184 & 3.01102 & 1.11602 \\
\hline Q9HCN4 & GPN-loop GTPase 1 & GPN1 & 0.535254 & 0.668491 & 1.38373 \\
\hline Q9NW64 & Pre-mRNA-splicing factor RBM22 ${ }^{* *}$ & RBM22 & 1.15759 & 1.93592 & 1.47532 \\
\hline Q9H3P2 & Negative elongation factor $A$ & NELFA & 1.54779 & 2.50404 & 2.02116 \\
\hline Q8WX92 & Negative elongation factor B & NELFB & 1.47736 & 2.75735 & 1.84038 \\
\hline Q8IXH7 & Negative elongation factor C/D & NELFCD & 1.54033 & 2.70383 & 1.18886 \\
\hline
\end{tabular}

\# proteins highlighted with color were tested in importin 13 overexpression experiments with bold and underlined proteins changing their subcellular distribution upon importin 13 coexpression: red: proteins enriched for Imp13/Imp13+Ran and Imp13/Imp13+Ubc9.; blue: proteins enriched only for Imp13/Imp13+Ran; orange: proteins enriched only for Imp13/Imp13+Ubc9; green: proteins enriched for Imp13/Imp13+Ran and Imp13/Imp13+Ubc9 in two out of three SILAC replicates; proteins labeled bold and black are known importin 13 import cargoes and the known export cargo elF1A

*right sided, one sample t-test $(p \leq 0.01)$, which tested the hypothesis that the log 2 SILAC ratios were not equal to the value zero

** proteins were localized to the cytoplasm and not the nucleus upon importin 13 coexpression 
Table S7: List of proteins identified as ambiguous importin 13 cargoes in SILAC screen (see section 3.3.3 for experimental details)

\begin{tabular}{|c|c|c|c|c|c|c|}
\hline $\begin{array}{l}\text { Uniprot } \\
\text { ID }\end{array}$ & Protein Name & Gene & $\begin{array}{l}\text { Iog2(Imp13+Ran/ } \\
\text { Imp13) }\end{array}$ & $\begin{array}{l}\text { Iog2(Imp13+Ran/ } \\
\text { Imp13+Ubc9) }\end{array}$ & $\begin{array}{l}\text { Iog2(Imp13I } \\
\text { Imp13+Ran) }\end{array}$ & $\begin{array}{l}\text { log2(Imp13/ } \\
\text { Imp13+Ubc9) }\end{array}$ \\
\hline P47813 & Eukaryotic translation initiation factor $1 \mathrm{~A}$ & EIF1AX & 3.25237 & 4.8423 & -3.25237 & 1.42849 \\
\hline Q96HC4 & $\begin{array}{l}\text { PDZ and LIM domain protein } 5 \\
\text { 6-phosphofructo-2-kinase/fructose-2,6- }\end{array}$ & PDLIM5 & 2.97543 & 3.80419 & -2.97543 & 0.955176 \\
\hline 060825 & bisphosphatase 2 & PFKFB2 & 3.29633 & 3.83705 & -3.29633 & 1.00394 \\
\hline E7EV99 & Alpha-adducin & ADD1 & 0.922004 & 1.80595 & -0.922004 & 1.30584 \\
\hline P26196 & Probable ATP-dependent RNA helicase DDX6 & DDX6 & 0.713644 & 1.66241 & -0.713644 & 1.21929 \\
\hline P61978 & Heterogeneous nuclear ribonucleoprotein $\mathrm{K}$ & HNRNPK & 1.19892 & 2.05797 & -1.19892 & 1.3447 \\
\hline Q8ND56 & Protein LSM14 homolog A & LSM14A & 0.890691 & 2.15422 & -0.890691 & 1.6105 \\
\hline Q9P270 & SLAIN motif-containing protein 2 & SLAIN2 & 1.01011 & 1.95006 & -1.01011 & 1.12969 \\
\hline P06493 & Cyclin-dependent kinase 1 & CDK1 & 1.34662 & 2.27739 & -1.34662 & 0.738885 \\
\hline B7Z5N5 & Mothers against decapentaplegic homolog & SMAD2 & 0.961146 & 2.27677 & -0.961146 & 1.53707 \\
\hline B7Z7P8 & Eukaryotic peptide chain release factor subunit 1 & ETF1 & 1.00667 & 1.57229 & -1.00667 & 0.872366 \\
\hline P12956 & $\underline{X \text {-ray repair cross-complementing protein } 6}$ & XRCC6 & 0.977419 & 1.37652 & -0.977419 & 0.841513 \\
\hline P50548 & $\begin{array}{l}\text { ETS domain-containing transcription factor ERF } \\
\text { Activated RNA polymerase II transcriptional }\end{array}$ & ERF & 0.875577 & 2.19828 & -0.875577 & 1.60979 \\
\hline P53999 & coactivator p15 & SUB1 & 1.50329 & 4.967 & -1.50329 & 2.80937 \\
\hline Q00403 & Transcription initiation factor IIB & GTF2B & 1.80555 & 3.2855 & -1.80555 & 1.67293 \\
\hline Q92900 & Regulator of nonsense transcripts 1 & UPF1 & 1.15652 & 2.18872 & -1.15652 & 1.3723 \\
\hline
\end{tabular}

\# protein labeled bold and black is a known importin 13 export cargo; protein labeled in green and underlined was validated as an export cargo in importin 13 overexpression experiments

Table S8: List of potential importin 13 export cargoes identified in SILAC screen $(\log 2(\operatorname{Imp13+Ran} / \mathrm{Imp13})>0.5$ for all three experimental replicates, see section 3.3.3 for experimental details)

\begin{tabular}{|c|c|c|c|c|c|}
\hline $\begin{array}{l}\text { Uniprot } \\
\text { ID }\end{array}$ & Protein Name & Gene & $\begin{array}{l}\log 2(\operatorname{Imp} 13+R a n / \\
\text { Imp13) }\end{array}$ & $\begin{array}{l}\text { log2(Imp13+Ran/ } \\
\text { Imp13+Ubc9) }\end{array}$ & $\begin{array}{l}-\log \\
\text { ( } p \text { value) }\end{array}$ \\
\hline \multicolumn{6}{|c|}{$\log 2(\operatorname{Imp} 13+\operatorname{Ran} / \operatorname{lmp} 13) \geq 3.0$} \\
\hline P43487 & Ran-specific GTPase-activating protein & RANBP1 & 5.38648 & 5.32265 & 1.81783 \\
\hline 000442 & RNA 3-terminal phosphate cyclase & RTCA & 4.57597 & 5.59694 & 2.50708 \\
\hline P39748 & Flap endonuclease 1 & FEN1 & 4.43566 & 3.9402 & 1.84603 \\
\hline Q6IPR3 & tRNA wybutosine-synthesizing protein 3 homolog & TYW3 & 4.26835 & 4.34712 & 2.42506 \\
\hline P27695 & DNA-(apurinic or apyrimidinic site) lyase & APEX1 & 4.12269 & 3.50792 & 2.09237 \\
\hline Q9H3H3 & UPF0696 protein C11orf68 & C11orf68 & 4.07843 & 3.406 & 1.75539 \\
\hline Q86W56 & Poly(ADP-ribose) glycohydrolase & PARG & 3.89607 & 2.94416 & 2.03045 \\
\hline P37108 & Signal recognition particle $14 \mathrm{kDa}$ protein & SRP14 & 3.77885 & 4.36023 & 3.05751 \\
\hline Q96PZ0 & Pseudouridylate synthase 7 homolog & PUS7 & 3.7069 & 2.6616 & 1.93934 \\
\hline P35241 & Radixin & $\mathrm{RDX}$ & 3.61814 & 3.22884 & 2.60103 \\
\hline 094829 & Importin-13 & IPO13 & 3.56757 & 2.26277 & 1.28052 \\
\hline Q08J23 & tRNA (cytosine(34)-C(5))-methyltransferase & NSUN2 & 3.56745 & 2.93758 & 2.29027 \\
\hline P54577 & Tyrosine--tRNA ligase, cytoplasmic & YARS & 3.56152 & 3.04854 & 2.04963 \\
\hline P84077 & ADP-ribosylation factor 1 & ARF1;ARF3 & 3.54006 & 3.5376 & 2.09902 \\
\hline Q32Q12 & Nucleoside diphosphate kinase & NME1-NME2 & 3.46541 & 3.61361 & 2.03003 \\
\hline P08237 & 6-phosphofructokinase, muscle type & PFKM & 3.44466 & 3.14856 & 1.96288 \\
\hline Q8WWH5 & Probable tRNA pseudouridine synthase 1 & TRUB1 & 3.41881 & 3.07742 & 2.07842 \\
\hline B5MCF9 & Pescadillo homolog & PES1 & 3.37922 & 3.12792 & 1.77702 \\
\hline F8VZ49 & Heterogeneous nuclear ribonucleoprotein A1 & HNRNPA1 & 3.33691 & 3.12131 & 1.60299 \\
\hline 060825 & 6-phosphofructo-2-kinase/fructose-2,6-bisphosphatase 2 & PFKFB2 & 3.29633 & 3.83705 & 1.98737 \\
\hline P47813 & Eukaryotic translation initiation factor $1 \mathrm{~A}$ & EIF1A & 3.25237 & 4.8423 & 4.24736 \\
\hline \multicolumn{6}{|c|}{$\log 2(\operatorname{Imp} 13+\operatorname{Ran} / \mathrm{Imp} 13) \geq 1.5$} \\
\hline Q96HC4 & PDZ and LIM domain protein 5 & PDLIM5 & 2.97543 & 3.80419 & 2.37063 \\
\hline HOYA96 & Heterogeneous nuclear ribonucleoprotein D0 & HNRNPD & 2.96732 & 2.53505 & 2.24646 \\
\hline
\end{tabular}




\begin{tabular}{|c|c|c|c|c|c|}
\hline $\begin{array}{l}\text { Uniprot } \\
\text { ID }\end{array}$ & Protein Name & Gene & $\begin{array}{l}\log 2(\operatorname{Imp} 13+R a n / \\
\text { Imp13) }\end{array}$ & $\begin{array}{l}\log 2(\operatorname{Imp} 13+R a n / \\
\text { Imp13+Ubc9) }\end{array}$ & $\begin{array}{l}-\log \\
(p \text { value })^{*}\end{array}$ \\
\hline P26038 & Moesin & MSN & 2.95718 & 3.0089 & 1.27479 \\
\hline 095347 & Structural maintenance of chromosomes protein 2 & SMC2 & 2.93072 & 2.13327 & 2.17994 \\
\hline P13010 & $\underline{X \text {-ray repair cross-complementing protein } 5}$ & XRCC5 & 2.82663 & 1.82907 & 2.72781 \\
\hline Q6VY07 & Phosphofurin acidic cluster sorting protein 1 & PACS1 & 2.82146 & 2.83474 & 2.62546 \\
\hline Q7Z4S6 & Kinesin-like protein KIF21A & KIF21A & 2.78632 & 2.54737 & 2.35845 \\
\hline 075116 & Rho-associated protein kinase 2 & ROCK2 & 2.78412 & 2.10553 & 2.80105 \\
\hline Q8TBB5 & Kelch domain-containing protein 4 & KLHDC4 & 2.77381 & 2.83467 & 1.84029 \\
\hline Q9NTK5 & Obg-like ATPase 1 & OLA1 & 2.76445 & 2.21738 & 3.92603 \\
\hline Q9UK59 & Lariat debranching enzyme & DBR1 & 2.6774 & 2.10752 & 2.66408 \\
\hline Q9BQ52 & Zinc phosphodiesterase ELAC protein 2 & ELAC2 & 2.60043 & 1.21415 & 1.88731 \\
\hline O75534 & Cold shock domain-containing protein E1 & CSDE1 & 2.52311 & 2.63836 & 1.87994 \\
\hline P15311 & Ezrin & EZR & 2.48226 & 1.67527 & 1.48324 \\
\hline Q9Y3F4 & Serine-threonine kinase receptor-associated protein & STRAP & 2.40696 & 2.44099 & 1.68925 \\
\hline Q9BPX3 & Condensin complex subunit 3 & NCAPG & 2.29883 & 2.17429 & 2.48105 \\
\hline Q5VTE0 & Putative elongation factor 1-alpha-like 3 & EEF1A1P5 & 2.29715 & 1.93896 & 1.87181 \\
\hline Q15631 & Translin & TSN & 2.2224 & 2.3705 & 2.27251 \\
\hline P09661 & U2 small nuclear ribonucleoprotein A & SNRPA1 & 2.21552 & 2.5631 & 1.62974 \\
\hline P25205 & DNA replication licensing factor $\mathrm{MCM} 3$ & МСM3 & 2.2095 & 1.8214 & 2.44116 \\
\hline P36915 & Guanine nucleotide-binding protein-like 1 & GNL1 & 2.14833 & 2.18778 & 1.93622 \\
\hline P24534 & Elongation factor 1-beta & EEF1B2 & 1.97594 & 1.76944 & 1.40369 \\
\hline Q96K76 & Ubiquitin carboxyl-terminal hydrolase 47 & USP47 & 1.95828 & 0.601034 & 2.78035 \\
\hline Q7KZF4 & Staphylococcal nuclease domain-containing protein 1 & SND1 & 1.93858 & 0.886815 & 2.02139 \\
\hline Q9NRF8 & CTP synthase 2 & CTPS2 & 1.91466 & -0.496447 & 2.31617 \\
\hline Q66K74 & Microtubule-associated protein 1S & MAP1S & 1.89109 & 1.42357 & 2.27209 \\
\hline 000203 & AP-3 complex subunit beta-1 & AP3B1 & 1.86118 & 0.904195 & 1.35445 \\
\hline P20290 & Transcription factor BTF3 & BTF3 & 1.82755 & 1.8911 & 1.59095 \\
\hline Q00403 & Transcription initiation factor IIB & GTF2B & 1.80555 & 3.2855 & 1.75203 \\
\hline Q9UHD8 & Septin-9 & SEPT9 & 1.75664 & 1.8294 & 1.67951 \\
\hline HOYBP1 & Focal adhesion kinase 1 & PTK2 & 1.73248 & 1.95922 & 2.19702 \\
\hline Q9BV44 & THUMP domain-containing protein 3 & THUMPD3 & 1.70402 & 1.51059 & 2.36607 \\
\hline P41214 & Eukaryotic translation initiation factor $2 \mathrm{D}$ & EIF2D & 1.63737 & 1.46164 & 1.72077 \\
\hline P26639 & Threonine--tRNA ligase, cytoplasmic & TARS & 1.60822 & 1.18292 & 0.947564 \\
\hline E9PK01 & Elongation factor 1-delta & EEF1D & 1.54923 & 0.900679 & 1.62074 \\
\hline P53999 & Activated RNA polymerase II transcriptional coactivator p15 & SUB1 & 1.50329 & 4.967 & 1.49769 \\
\hline \multicolumn{6}{|c|}{$\log 2(\operatorname{Imp} 13+\operatorname{Ran} / \operatorname{Imp} 13) \geq 0.5$} \\
\hline P26641 & Elongation factor 1-gamma & EEF1G & 1.49928 & 0.940673 & 1.63639 \\
\hline Q14008 & Cytoskeleton-associated protein 5 & CKAP5 & 1.48043 & 1.59961 & 1.7579 \\
\hline Q01813 & 6-phosphofructokinase type C & PFKP & 1.47333 & 1.66786 & 1.54622 \\
\hline P19338 & Nucleolin & $\mathrm{NCL}$ & 1.4483 & 0.362352 & 2.02784 \\
\hline P40227 & T-complex protein 1 subunit zeta & CCT6A & 1.43843 & 1.00277 & 1.8217 \\
\hline Q92499 & ATP-dependent RNA helicase DDX1 & DDX1 & 1.42124 & 0.879824 & 2.54251 \\
\hline Q96CX2 & BTB/POZ domain-containing protein KCTD12 & KCTD12 & 1.39005 & -1.47452 & 2.00427 \\
\hline P27448 & MAP/microtubule affinity-regulating kinase 3 & MARK3 & 1.38863 & 1.12089 & 1.75108 \\
\hline P17987 & T-complex protein 1 subunit alpha & TCP1 & 1.3804 & 0.963823 & 1.74164 \\
\hline E9PC69 & Serine/threonine-protein kinase MARK2 & MARK2 & 1.37177 & 1.51294 & 1.69682 \\
\hline P06493 & Cyclin-dependent kinase 1 & CDK1 & 1.34662 & 2.27739 & 1.64412 \\
\hline P28482 & Mitogen-activated protein kinase 1 & MAPK1 & 1.345 & 1.27727 & 1.42906 \\
\hline Q9HB71 & Calcyclin-binding protein & CACYBP & 1.33717 & 1.79655 & 1.60171 \\
\hline Q9UDY4 & DnaJ homolog subfamily B member 4 & DNAJB4 & 1.32143 & 0.922722 & 1.44451 \\
\hline P34932 & Heat shock $70 \mathrm{kDa}$ protein 4 & HSPA4 & 1.30134 & 0.723749 & 2.41801 \\
\hline F8VZJ2 & Nascent polypeptide-associated complex subunit alpha & NACA & 1.29685 & 0.884699 & 1.20009 \\
\hline P62937 & Peptidyl-prolyl cis-trans isomerase A & PPIA & 1.27194 & 2.3087 & 1.63458 \\
\hline P27816 & Microtubule-associated protein 4;Microtubule-associated protein & MAP4 & 1.23621 & 0.871172 & 1.73817 \\
\hline F8VPF3 & Myosin light polypeptide 6 & MYL6 & 1.22701 & 1.53608 & 1.13832 \\
\hline Q3KQU3 & MAP7 domain-containing protein 1 & MAP7D1 & 1.22021 & 1.0111 & 1.40087 \\
\hline Q06830 & Peroxiredoxin-1 & PRDX1 & 1.19912 & 1.609 & 1.87588 \\
\hline P50990 & T-complex protein 1 subunit theta & ССТ8 & 1.19894 & 0.809329 & 1.73926 \\
\hline
\end{tabular}




\begin{tabular}{|c|c|c|c|c|c|}
\hline $\begin{array}{l}\text { Uniprot } \\
\text { ID }\end{array}$ & Protein Name & Gene & $\begin{array}{l}\log 2(\operatorname{Imp} 13+R a n / \\
\text { Imp13) }\end{array}$ & $\begin{array}{l}\log 2(\operatorname{Imp} 13+R a n / \\
\text { Imp13+Ubc9) }\end{array}$ & $\begin{array}{l}-\log \\
(p \text { value })^{*}\end{array}$ \\
\hline P61978 & Heterogeneous nuclear ribonucleoprotein $\mathrm{K}$ & HNRNPK & 1.19892 & 2.05797 & 3.26672 \\
\hline B4E1C5 & Histidine--tRNA ligase, cytoplasmic & HARS & 1.18682 & 0.84494 & 1.13876 \\
\hline P46940 & Ras GTPase-activating-like protein IQGAP1 & IQGAP1 & 1.17839 & 0.84498 & 1.75069 \\
\hline Q15366 & Poly(rC)-binding protein 2 & PCBP2 & 1.16694 & 0.795609 & 1.33161 \\
\hline Q92900 & Regulator of nonsense transcripts 1 & UPF1 & 1.15652 & 2.18872 & 1.49077 \\
\hline P17812 & CTP synthase 1 & CTPS1 & 1.1524 & -0.202184 & 2.33023 \\
\hline P50991 & T-complex protein 1 subunit delta & CCT4 & 1.12879 & 0.681773 & 1.73994 \\
\hline P78371 & T-complex protein 1 subunit beta & ССТ2 & 1.09746 & 0.654692 & 1.60643 \\
\hline P48643 & T-complex protein 1 subunit epsilon & ССT5 & 1.08008 & 0.74469 & 1.64779 \\
\hline P49327 & Fatty acid synthase & FASN & 1.06924 & 0.653674 & 1.33252 \\
\hline Q92598 & Heat shock protein $105 \mathrm{kDa}$ & HSPH1 & 1.06503 & 0.487717 & 2.35444 \\
\hline Q9P270 & SLAIN motif-containing protein 2 & SLAIN2 & 1.01011 & 1.95006 & 2.64552 \\
\hline B7Z7P8 & Eukaryotic peptide chain release factor subunit 1 & ETF1 & 1.00667 & 1.57229 & 1.97514 \\
\hline Q9Y4E8 & Ubiquitin carboxyl-terminal hydrolase 15 & USP15 & 0.979349 & 0.217313 & 2.22068 \\
\hline P12956 & $\underline{\mathrm{X} \text {-ray repair cross-complementing protein } 6}$ & XRCC6 & 0.977419 & 1.37652 & 1.40745 \\
\hline P19525 & Interferon-induced, double-stranded RNA-activated protein kinase & EIF2AK2 & 0.97668 & 0.410077 & 2.54683 \\
\hline 014744 & Protein arginine $\mathrm{N}$-methyltransferase 5 & PRMT5 & 0.967085 & 0.708434 & 1.96941 \\
\hline Q15365 & Poly(rC)-binding protein 1 & PCBP1 & 0.964946 & 0.634472 & 2.96652 \\
\hline B7Z5N5 & Mothers against decapentaplegic homolog 2 & SMAD2 & 0.961146 & 2.27677 & 1.96035 \\
\hline E7EV99 & Alpha-adducin & ADD1 & 0.922004 & 1.80595 & 3.00514 \\
\hline Q8ND56 & Protein LSM14 homolog A & LSM14A & 0.890691 & 2.15422 & 3.56774 \\
\hline 060506 & Heterogeneous nuclear ribonucleoprotein Q & SYNCRIP & 0.883583 & 0.40863 & 1.48999 \\
\hline P50548 & ETS domain-containing transcription factor ERF & ERF & 0.875577 & 2.19828 & 1.38588 \\
\hline P16989 & Y-box-binding protein 3 & YBX3 & 0.825159 & 0.469257 & 2.04904 \\
\hline Q01105 & Protein SET & SET & 0.81555 & 0.0277848 & 2.56819 \\
\hline Q9BRS2 & Serine/threonine-protein kinase RIO1 & RIOK1 & 0.802329 & 1.13405 & 2.09427 \\
\hline P78527 & DNA-dependent protein kinase catalytic subunit & PRKDC & 0.724773 & 0.91282 & 1.92342 \\
\hline 060524 & Nuclear export mediator factor NEMF & NEMF & 0.721399 & 0.0807594 & 2.1625 \\
\hline P26196 & Probable ATP-dependent RNA helicase DDX6 & DDX6 & 0.713644 & 1.66241 & 2.0097 \\
\hline 000178 & GTP-binding protein 1 & GTPBP1 & 0.694846 & 1.07533 & 1.90228 \\
\hline K7EN82 & Glycylpeptide N-tetradecanoyltransferase 1 & NMT1 & 0.651957 & -0.137875 & 2.2303 \\
\hline \multicolumn{6}{|c|}{$\log 2(\operatorname{Imp} 13+R a n / I m p 13) \geq 0.5$ for only 2 out of 3 replicates } \\
\hline Q9BQA1 & Methylosome protein 50 & WDR77 & 0.946335 & 0.381559 & 1.04849 \\
\hline
\end{tabular}

\# proteins highlighted with color were tested in importin 13 overexpression experiments with bold and underlined proteins changing their subcellular distribution upon importin 13 coexpression: blue: proteins with an average $\log 2(\operatorname{Imp} 13+R a n / I m p 13) \geq 3.0$; orange: proteins with an average log2(Imp13+Ran/Imp13) $\geq 1.5$; green: proteins with an average $\log 2(\operatorname{Imp} 13+\mathrm{Ran} / \mathrm{Imp} 13) \geq 0.5$; proteins labeled bold and black are known importin 13 export cargoes

*right sided, one sample t-test $(p \leq 0.01)$, which tested the hypothesis that the log2 SILAC ratios were not equal to the value zero

Table S9: Importin 13 cargo candidates identified in SILAC screen and by mass spectrometry in section 3.3.1 (see section 3.3.1 and 3.3.3 for experimental details)

\begin{tabular}{lll}
\hline Uniprot ID & Protein Name & Gene \\
\hline & & \multicolumn{1}{c}{ Identified as export cargoes } \\
\hline P49327 & Fatty acid synthase & FASN \\
P08237 & ATP-dependent 6-phosphofructokinase, muscle type & PFKM \\
P34932 & Heat shock 70 kDa protein 4 & HSPA4 \\
P17812 & CTP synthase 1 & CTPS1 \\
P17987 & T-complex protein 1 subunit alpha & TCP1 \\
Q01813 & ATP-dependent 6-phosphofructokinase, platelet type & PFKP \\
Q9Y4E8 & Ubiquitin carboxyl-terminal hydrolase 15 & USP15 \\
P47813 & Eukaryotic translation initiation factor 1A & EIF1AX \\
P84077 & ADP-ribosylation factor 1 & ARF1 \\
Q92598 & Heat shock protein 105 kDa & HSPH1 \\
Q15366 & Poly(rC)-binding protein 2 & PCBP2
\end{tabular}




\begin{tabular}{|c|c|c|}
\hline Uniprot ID & Protein Name & Gene \\
\hline P19338 & Nucleolin & NCL \\
\hline 000178 & GTP-binding protein 1 & GTPBP1 \\
\hline Q7KZF4 & Staphylococcal nuclease domain-containing protein 1 & SND1 \\
\hline Q9Y3F4 & Serine-threonine kinase receptor-associated protein & STRAP \\
\hline Q9UDY4 & DnaJ homolog subfamily B member 4 & DNAJB4 \\
\hline Q9BV44 & THUMP domain-containing protein 3 & THUMPD3 \\
\hline O75116 & Rho-associated protein kinase 2 & ROCK2 \\
\hline 060506 & Heterogeneous nuclear ribonucleoprotein $\mathrm{Q}$ & SYNCRIP \\
\hline \multicolumn{3}{|c|}{ Identified as import cargoes } \\
\hline Q92538 & Golgi-specific brefeldin A-resistance guanine nucleotide exchange factor 1 & GBF1 \\
\hline Q7Z3U7 & Protein MON2 homolog & MON2 \\
\hline Q9NRF9 & DNA polymerase epsilon subunit 3 & POLE3 \\
\hline P61326 & Protein mago nashi homolog & MAGOH \\
\hline O75420 & GRB10-interacting GYF protein 1 & GIGYF1 \\
\hline Q9NRG0 & Chromatin accessibility complex protein 1 & CHRAC1 \\
\hline Q9Y4H2 & Insulin receptor substrate 2 & IRS2 \\
\hline Q8WUF5 & RelA-associated inhibitor & PPP1R13L \\
\hline P27540 & Aryl hydrocarbon receptor nuclear translocator & ARNT \\
\hline Q7Z460 & CLIP-associating protein 1 & CLASP1 \\
\hline Q16204 & Coiled-coil domain-containing protein 6 & CCDC6 \\
\hline Q9P1Y5 & Calmodulin-regulated spectrin-associated protein 3 & CAMSAP3 \\
\hline O15084 & Serine/threonine-protein phosphatase 6 regulatory ankyrin repeat subunit $A$ & ANKRD28 \\
\hline Q8WX93 & Palladin & PALLD \\
\hline Q6PGP7 & Tetratricopeptide repeat protein 37 & TTC37 \\
\hline Q8IUD2 & ELKS/Rab6-interacting/CAST family member 1 & ERC1 \\
\hline 000743 & Serine/threonine-protein phosphatase 6 catalytic subunit & PPP6C \\
\hline Q92974 & Rho guanine nucleotide exchange factor 2 & ARHGEF2 \\
\hline C9JZR2 & Catenin delta-1 & CTNND1 \\
\hline Q15477 & Helicase SKI2W & SKIV2L \\
\hline Q5T4S7 & E3 ubiquitin-protein ligase UBR4 & UBR4 \\
\hline Q7L2H7 & Eukaryotic translation initiation factor 3 subunit $\mathrm{M}$ & EIF3M \\
\hline P39023 & $60 S$ ribosomal protein $\mathrm{L} 3$ & RPL3 \\
\hline Q9GZS3 & WD repeat-containing protein 61 & WDR61 \\
\hline P55884 & Eukaryotic translation initiation factor 3 subunit B & EIF3B \\
\hline Q16513 & Serine/threonine-protein kinase N2 & PKN2 \\
\hline Q92888 & Rho guanine nucleotide exchange factor 1 & ARHGEF1 \\
\hline Q96JG6 & Syndetin & VPS50 \\
\hline Q7Z2W4 & Zinc finger $\mathrm{CCCH}$-type antiviral protein 1 & ZC3HAV1 \\
\hline P61981 & 14-3-3 protein gamma & YWHAG \\
\hline P23258 & Tubulin gamma-1 chain & TUBG1 \\
\hline B1ALK7 & Rho guanine nucleotide exchange factor 7 & ARHGEF7 \\
\hline Q9BYX4 & Interferon-induced helicase C domain-containing protein 1 & $\mathrm{IFIH} 1$ \\
\hline Q9NR33 & DNA polymerase epsilon subunit 4 & POLE4 \\
\hline Q13347 & Eukaryotic translation initiation factor 3 subunit I & EIF3I \\
\hline P60228 & Eukaryotic translation initiation factor 3 subunit $\mathrm{E}$ & EIF3E \\
\hline Q86UU1 & Pleckstrin homology-like domain family B member 1 & PHLDB1 \\
\hline Q5VIR6 & Vacuolar protein sorting-associated protein 53 homolog & VPS53 \\
\hline Q9Y530 & O-acetyl-ADP-ribose deacetylase 1 & OARD1 \\
\hline P31946 & 14-3-3 protein beta/alpha & YWHAB \\
\hline Q9Y262 & Eukaryotic translation initiation factor 3 subunit $L$ & EIF3L \\
\hline Q12800 & Alpha-globin transcription factor $\mathrm{CP} 2$ & TFCP2 \\
\hline H0YM23 & Ankyrin repeat domain-containing protein 17 & ANKRD17 \\
\hline Q14919 & Dr1-associated corepressor & DRAP1 \\
\hline Q01658 & Protein Dr1 & DR1 \\
\hline B1AKN7 & Nuclear factor 1 & NFIA \\
\hline Q8TEW0 & Partitioning defective 3 homolog & PARD3 \\
\hline
\end{tabular}


Table S10: Importin 13 cargo candidates identified in SILAC screen and the Kimura et al., 2017 study (76) (see section 3.3.3 for experimental details)

\begin{tabular}{|c|c|c|}
\hline Uniprot ID & Protein Name & Gene \\
\hline \multicolumn{3}{|c|}{ Identified as ambiguous importin 13 cargoes } \\
\hline Q96HC4 & PDZ and LIM domain protein 5 & PDLIM5 \\
\hline P47813 & Eukaryotic translation initiation factor $1 \mathrm{~A}$ & EIF1AX \\
\hline P26196 & Probable ATP-dependent RNA helicase DDX6 & DDX6 \\
\hline P06493 & Cyclin-dependent kinase 1 & CDK1 \\
\hline \multicolumn{3}{|c|}{ Identified as importin 13 export cargoes } \\
\hline Q9Y3F4 & Serine-threonine kinase receptor-associated protein & STRAP \\
\hline Q9NTK5 & Obg-like ATPase 1 & OLA1 \\
\hline P16989 & Y-box-binding protein 3 & YBX3 \\
\hline P62937 & Peptidyl-prolyl cis-trans isomerase A & PPIA \\
\hline P24534 & Elongation factor 1-beta & EEF1B2 \\
\hline Q15366 & Poly(rC)-binding protein 2 & PCBP2 \\
\hline P26641 & Elongation factor 1-gamma & EEF1G \\
\hline P20290 & Transcription factor BTF3 & BTF3 \\
\hline Q9Y4E8 & Ubiquitin carboxyl-terminal hydrolase 15 & USP15 \\
\hline P27816 & Microtubule-associated protein 4 & MAP4 \\
\hline P49327 & Fatty acid synthase & FASN \\
\hline \multicolumn{3}{|c|}{ Identified as importin 13 import cargoes } \\
\hline P52657 & Transcription initiation factor IIA subunit 2 & GTF2A2 \\
\hline P61326 & Protein mago nashi homolog & MAGOH \\
\hline Q9NRG0 & Chromatin accessibility complex protein 1 & CHRAC1 \\
\hline Q9NRF9 & DNA polymerase epsilon subunit 3 & POLE3 \\
\hline Q14247 & Src substrate cortactin & CTTN \\
\hline P61956 & Small ubiquitin-related modifier 2 & SUMO2 \\
\hline Q14157 & Ubiquitin-associated protein 2-like & UBAP2L \\
\hline Q9Y262 & Eukaryotic translation initiation factor 3 subunit $\mathrm{L}$ & EIF3L \\
\hline Q99613 & Eukaryotic translation initiation factor 3 subunit $\mathrm{C}$ & EIF3C \\
\hline Q14244 & Ensconsin & MAP7 \\
\hline P23588 & Eukaryotic translation initiation factor $4 \mathrm{~B}$ & EIF4B \\
\hline P78344 & Eukaryotic translation initiation factor 4 gamma 2 & EIF4G2 \\
\hline Q7Z2W4 & Zinc finger $\mathrm{CCCH}$-type antiviral protein 1 & ZC3HAV1 \\
\hline
\end{tabular}

\#: Bold and black: known importin 13 cargoes; bold and blue: proteins validated in this study as importin 13 cargo candidates using importin 13 overexpression assays; orange: proteins tested but not affected in importin 13 overexpression assays 

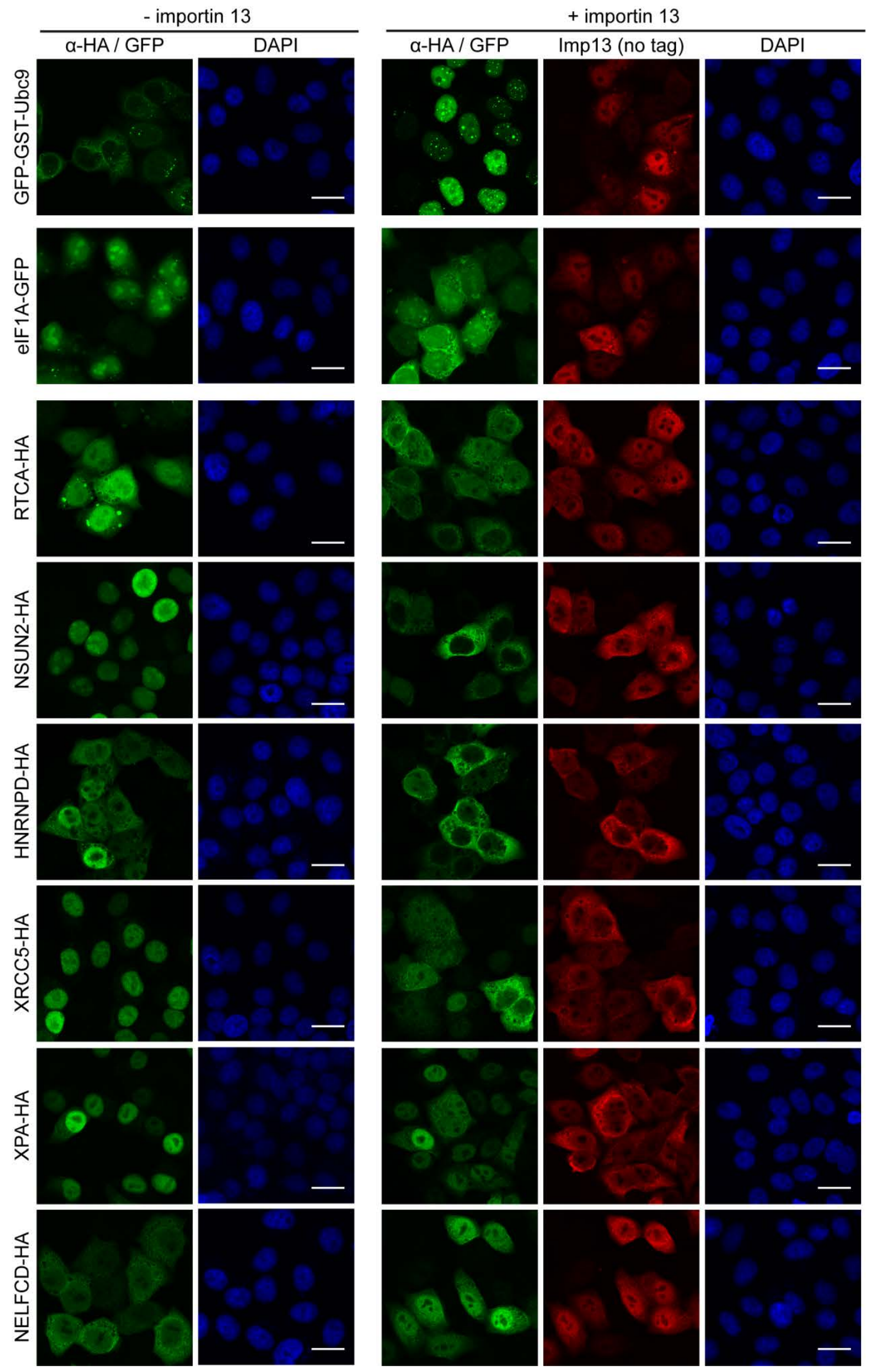
Figure S5: Effect of untagged importin 13 overexpression on the subcellular distribution of HA-tagged SILAC cargo candidates. HeLa P4 cells were transiently cotransfected with plasmids coding for HA-tagged proteins form the SILAC screen, GFP-GST-Ubc9 or elF1A-GFP and untagged importin 13 or an empty control vector (pcDNA3.1) using the calcium phosphate method. HA-tagged substrates and untagged importin 13 were visualized by indirect immunofluorescence with an anti-HA and anti-importin 13 antibody, respectively. Note that the endogenous importin 13 levels are not high enough to be detected by the importin 13 antibody. See section 3.3.3.4.3 for details. The scale bars correspond to $20 \mu \mathrm{m}$.

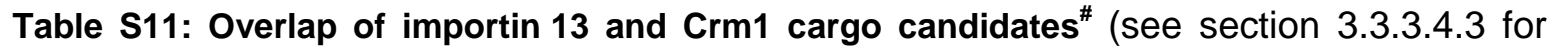
details)

\begin{tabular}{|c|c|c|}
\hline Uniprot ID & Protein Name & Gene \\
\hline \multicolumn{3}{|c|}{ Identified as ambiguous importin 13 cargoes } \\
\hline P50548 & ETS domain-containing transcription factor ERF & ERF \\
\hline P26196 & Probable ATP-dependent RNA helicase DDX6 & DDX6 \\
\hline Q8ND56 & Protein LSM14 homolog A & LSM14A \\
\hline Q96HC4 & PDZ and LIM domain protein 5 & PDLIM5 \\
\hline Q9P270 & SLAIN motif-containing protein 2 & SLAIN2 \\
\hline Q92900 & Regulator of nonsense transcripts 1 & UPF1 \\
\hline \multicolumn{3}{|c|}{ Identified as importin 13 export cargoes } \\
\hline Q9UHD8 & Septin-9 & SEPT9 \\
\hline 000203 & AP-3 complex subunit beta-1 & AP3B1 \\
\hline 075534 & Cold shock domain-containing protein E1 & CSDE1 \\
\hline Q9UK59 & Lariat debranching enzyme & DBR1 \\
\hline Q9NRF8 & CTP synthase 2 & CTPS2 \\
\hline P09661 & U2 small nuclear ribonucleoprotein $A^{\prime}$ & SNRPA1 \\
\hline Q8WWH5 & Probable tRNA pseudouridine synthase 1 & TRUB1 \\
\hline Q9Y3F4 & Serine-threonine kinase receptor-associated protein & STRAP \\
\hline Q96K76 & Ubiquitin carboxyl-terminal hydrolase 47 & USP47 \\
\hline Q9BRS2 & Serine/threonine-protein kinase RIO1 & RIOK1 \\
\hline \multicolumn{3}{|c|}{ Identified as importin 13 import cargoes } \\
\hline Q12800 & Alpha-globin transcription factor CP2 & TFCP2 \\
\hline Q7Z2W4 & Zinc finger $\mathrm{CCCH}$-type antiviral protein 1 & ZC3HAV1 \\
\hline Q13895 & Bystin & BYSL \\
\hline P49757 & Protein numb homolog & NUMB \\
\hline Q9Y6R0 & Numb-like protein & NUMBL \\
\hline Q9P0J7 & E3 ubiquitin-protein ligase KCMF1 & KCMF1 \\
\hline Q9UHR6 & Zinc finger HIT domain-containing protein 2 & ZNHIT2 \\
\hline Q14693 & Phosphatidate phosphatase LPIN1 & LPIN1 \\
\hline Q96F86 & Enhancer of mRNA-decapping protein 3 & EDC3 \\
\hline Q13501 & Sequestosome-1** & SQSTM1 \\
\hline P60228 & 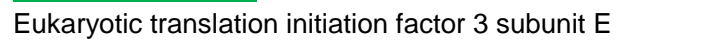 & EIF3E \\
\hline P78344 & Eukaryotic translation initiation factor 4 gamma 2 & EIF4G2 \\
\hline Q9Y4H2 & Insulin receptor substrate 2 & IRS2 \\
\hline P48729 & Casein kinase I isoform alpha & CSNK1A1 \\
\hline P23588 & Eukaryotic translation initiation factor $4 \mathrm{~B}$ & EIF4B \\
\hline Q3V6T2 & Girdin & CCDC88A \\
\hline Q15025 & TNFAIP3-interacting protein 1 & TNIP1 \\
\hline Q16204 & Coiled-coil domain-containing protein 6 & CCDC6 \\
\hline Q9Y262 & Eukaryotic translation initiation factor 3 subunit $L$ & EIF3L \\
\hline Q53EL6 & Programmed cell death protein $4^{* *}$ & PDCD4 \\
\hline P13984 & $\overline{\text { General transcription factor IIF subunit } 2^{* *}}$ & GTF2F2 \\
\hline Q99613 & Eukaryotic translation initiation factor 3 subunit $\mathrm{C}$ & $\mathrm{EIF} 3 \mathrm{C}$ \\
\hline 000743 & Serine/threonine-protein phosphatase 6 catalytic subunit & PPP6C \\
\hline Q14157 & Ubiquitin-associated protein 2-like & UBAP2L \\
\hline
\end{tabular}




\begin{tabular}{lll}
\hline Uniprot ID & Protein Name & Gene \\
\hline Q684P5 & Rap1 GTPase-activating protein 2 & RAP1GAP2 \\
Q8WUF5 & RelA-associated inhibitor & PPP1R13L \\
O75420 & GRB10-interacting GYF protein 1 & GIGYF1 \\
Q7Z460 & CLIP-associating protein 1 & CLASP1 \\
Q08AD1 & Calmodulin-regulated spectrin-associated protein 2 & CAMSAP2 \\
P23258 & Tubulin gamma-1 chain & TUBG1 \\
Q9GZS3 & WD repeat-containing protein 61 & WDR61 \\
Q14C86 & GTPase-activating protein and VPS9 domain-containing protein 1 & GAPVD1 \\
P41743 & Protein kinase C iota type & PRKCI \\
Q92888 & Rho guanine nucleotide exchange factor 1 & ARHGEF1 \\
P61981 & 14-3-3 protein gamma & YWHAG \\
Q5T4S7 & E3 ubiquitin-protein ligase UBR4 & UBR4 \\
O00571 & ATP-dependent RNA helicase DDX3X & DDX3X \\
P36578 & 60S ribosomal protein L4 & RPL4 \\
Q8IV48 & 3'-5' exoribonuclease 1 & ERI1 \\
Q86UU1 & Pleckstrin homology-like domain family B member 1 & PHLDB1 \\
\hline
\end{tabular}

\# proteins labeled bold and black are known importin 13 cargoes; proteins highlighted with color were tested in importin 13 overexpression experiments with bold and underlined proteins changing their subcellular distribution upon importin 13 coexpression. Export candidates: orange: proteins with an average $\log 2$ (Imp13+Ran/Imp13) $\geq 1.5$. Import candidates: red: proteins enriched for Imp13/Imp13+Ran and Imp13/Imp13+Ubc9.; blue: proteins enriched only for Imp13/Imp13+Ran; orange: proteins enriched only for Imp13/Imp13+Ubc9; green: proteins enriched for Imp13/Imp13+Ran and Imp13/Imp13+Ubc9 in two out of three SILAC replicates.

** proteins were localized to the cytoplasm and not the nucleus upon importin 13 coexpression

Table S12: Overlap importin 13 and importin 5 cargo candidates ${ }^{\#}$ (see section 3.3.3.4.3 for details)

\begin{tabular}{|c|c|c|}
\hline Uniprot ID & Protein Name & Gene \\
\hline \multicolumn{3}{|c|}{ Identified as ambiguous importin 13 cargoes } \\
\hline Q8ND56 & Protein LSM14 homolog A & LSM14A \\
\hline P47813 & Eukaryotic translation initiation factor $1 \mathrm{~A}$ & EIF1AX \\
\hline Q96HC4 & PDZ and LIM domain protein 5 & PDLIM5 \\
\hline P06493 & Cyclin-dependent kinase 1 & CDK1 \\
\hline P26196 & Probable ATP-dependent RNA helicase DDX6 & DDX6 \\
\hline Q92900 & Regulator of nonsense transcripts 1 & UPF1 \\
\hline \multicolumn{3}{|c|}{ Identified as importin 13 export cargoes } \\
\hline Q14008 & Cytoskeleton-associated protein 5 & CKAP5 \\
\hline Q9Y3F4 & Serine-threonine kinase receptor-associated protein & STRAP \\
\hline P24534 & Elongation factor 1-beta & EEF1B2 \\
\hline P20290 & Transcription factor BTF3 & BTF3 \\
\hline P26641 & $\overline{\text { Elongation factor 1-gamma }}$ & EEF1G \\
\hline P43487 & Ran-specific GTPase-activating protein & RANBP1 \\
\hline Q3KQU3 & MAP7 domain-containing protein 1 & MAP7D1 \\
\hline P16989 & Y-box-binding protein 3 & YBX3 \\
\hline 000442 & RNA 3'-terminal phosphate cyclase & RTCA \\
\hline Q9NTK5 & Obg-like ATPase 1 & OLA1 \\
\hline Q15366 & Poly(rC)-binding protein 2 & PCBP2 \\
\hline P27816 & Microtubule-associated protein 4 & MAP4 \\
\hline Q8TBB5 & Kelch domain-containing protein 4 & KLHDC4 \\
\hline Q7KZF4 & Staphylococcal nuclease domain-containing protein 1 & SND1 \\
\hline \multicolumn{3}{|c|}{ Identified as importin 13 import cargoes } \\
\hline P78344 & Eukaryotic translation initiation factor 4 gamma 2 & EIF4G2 \\
\hline Q8WWI1 & LIM domain only protein 7 & LMO7 \\
\hline Q96SU4 & Oxysterol-binding protein-related protein 9 & OSBPL9 \\
\hline Q14157 & Ubiquitin-associated protein 2-like & UBAP2L \\
\hline P23588 & Eukaryotic translation initiation factor $4 \mathrm{~B}$ & EIF4B \\
\hline Q14247 & Src substrate cortactin & CTTN \\
\hline 000571 & ATP-dependent RNA helicase DDX3X & DDX3X \\
\hline Q9NSD9 & Phenylalanine--tRNA ligase beta subunit & FARSB \\
\hline
\end{tabular}


Appendix

\begin{tabular}{lll}
\hline Uniprot ID & Protein Name & Gene \\
\hline Q5T4S7 & E3 ubiquitin-protein ligase UBR4 & UBR4 \\
P60228 & Eukaryotic translation initiation factor 3 subunit E & EIF3E \\
Q99613 & Eukaryotic translation initiation factor 3 subunit C & EIF3C \\
Q9Y262 & Eukaryotic translation initiation factor 3 subunit L & EIF3L \\
\hline
\end{tabular}

\# proteins labeled bold and black are known importin 13 cargoes; proteins highlighted with color were tested in importin 13 overexpression experiments with bold and underlined proteins changing their subcellular distribution upon importin 13 coexpression. Export candidates: blue: proteins with an average $\log 2(\operatorname{Imp} 13+R a n / I m p 13) \geq 3.0$; orange: proteins with an average $\log 2(\operatorname{Imp} 13+R a n / I m p 13) \geq 1.5$. Import candidates: orange: proteins enriched only for Imp13/Imp13+Ubc9. 


\section{Abbreviations}

A549

aa

AP

APS

Arg

Arg-6: $\mathrm{HCl}$

Arg-10:HCl

Asp

ATP

bp

BSA

CDNA

Crm1

C-terminus

D

DAPI

DNA

DMEM

DMSO

dNTPs

DTT

E

E. coli

EDTA

ESI

EtOH

FG

FLAG

FRAP

GAP

GDP

GFP

Glu

GO

GST

GTP

h

HA

$\mathrm{HCl}$

HEAT

HEPES

His

HIV-1 transformed adenocarcinoma cell line

amino acid

aprotinin

ammonium persulfate

arginine

${ }^{13} \mathrm{C}_{6}$-L-arginine $\mathrm{HCl}$

${ }^{13} \mathrm{C}_{6}{ }^{15} \mathrm{~N}_{4}$-L-arginine $\mathrm{HCl}$

aspartic acid

adenosine-5-triphosphate

base pair

bovine serum albumin

complementary DNA

chromosome region maintenance 1

carboxy-terminus

aspartic acid

4',6-diamidino-2-phenylindole

desoxyribonucleic acid

Dulbecco's modified eagles medium

dimethyl sulfoxide

2'-desoxynucleoside-5'-triphosphate

dithiothreitol

glutamic acid

Escherichia coli

ethylenediaminetetraacetic acid

electrospray ionization

ethanol

phenylalanine glycine

polypeptide protein tag with the sequence DYKDDDDK

fluorescence recovery after photobleaching

GTPase-activating protein

guanosine-5'-diphosphate

green fluorescent protein

glutamic acid

Gene Ontology

glutathione S-transferase

guanosine-5'-triphosphate

heavy (SILAC)

hemagglutinin

hydrochloric acid

Huntingtin Elongation Factor A Subunit Tor

2-[4-(2-hydroxyethyl)piperazin-1-yl]ethanesulfonic acid

histidine tag

human immunodeficiency virus type 1 


\begin{tabular}{|c|c|}
\hline hnRNP & heterogeneous nuclear ribonucleoproteins \\
\hline HRP & horseradish peroxidase \\
\hline IF & immunofluorescence \\
\hline Imp13 & importin 13 \\
\hline IPO13 & importin 13 gene \\
\hline IPTG & isopropyl-beta-D-thiogalactopyranoside \\
\hline kDa & kilo dalton \\
\hline I & light (SILAC) \\
\hline LB & Luria-Bertani \\
\hline LC & liquid chromatography \\
\hline LMB & Leptomycin B \\
\hline $\mathrm{m}$ & medium (SILAC) \\
\hline M9 & PY-NLS initially described for hnRNPA1 \\
\hline MCS & multiple cloning site \\
\hline mock & control treated sample \\
\hline MS & mass spectrometry \\
\hline MW & molecular weight \\
\hline MWCO & molecular weight cut-off \\
\hline $\mathrm{m} / \mathrm{z}$ & mass-to-charge ratio \\
\hline LC & liquid chromatography \\
\hline LC-MS & liquid chromatography - mass spectrometry \\
\hline LP & leupeptin, pepstatin \\
\hline Lys-4D:2HCl & 4,4,5,5-D4-L-Lysine $2 \mathrm{HCl}$ \\
\hline Lys-8: $\mathrm{HCl}$ & ${ }^{13} \mathrm{C}_{6}{ }^{15} \mathrm{~N}_{2}$-L-lysine $\mathrm{HCl}$ \\
\hline NA & numerical aperture \\
\hline NE & nuclear envelope \\
\hline NES & nuclear export signal \\
\hline NFAT & nuclear factor of activated T-cells \\
\hline NLS & nuclear localization signal \\
\hline NPC & nuclear pore complex \\
\hline NT & non targeting \\
\hline N-terminus & amino terminus \\
\hline NTF2 & nuclear transport factor 2 \\
\hline Nup & nucleoporin \\
\hline PAGE & polyacrylamide gel electrophoresis \\
\hline PBS & phosphate buffered saline \\
\hline PCR & polymerase chain reaction \\
\hline PMSF & phenylmethylsulphonyl fluoride \\
\hline PLA & proximity ligation assay \\
\hline PY-NLS & $\begin{array}{l}\text { NLS with } \mathrm{RX}_{2-5} \mathrm{PY} \text { motif at its } \mathrm{C} \text {-terminus and either a } \\
\text { hydrophobic or basic motif at its N-terminus }\end{array}$ \\
\hline $\mathrm{R}$ & arginine \\
\hline Ran & Ras-related nuclear protein \\
\hline RCC1 & regulator of chromatin condensation 1 \\
\hline RFP & red fluorescent protein \\
\hline
\end{tabular}




$\begin{array}{ll}\text { RNA } & \text { ribonucleic acid } \\ \text { RNAi } & \text { RNA interference } \\ \text { RP } & \text { reversed phase } \\ \text { rpm } & \text { rotations per minute } \\ \text { S. cerevisiae } & \text { Saccharomyces cerevisiae } \\ \text { SDS } & \text { sodium dodecyl sulphate } \\ \text { SILAC } & \text { stable isotope labeling with amino acids in cell culture } \\ \text { SiRNA } & \text { small interfering RNA } \\ \text { SPN1 } & \text { snurportin 1 } \\ \text { SUMO } & \text { small ubiquitin-like modifier } \\ \text { SV40 } & \text { simian virus 40 } \\ \text { tev } & \text { TEV-protease cleavage site } \\ \text { TAE } & \text { Tris/ Acetate/ EDTA } \\ \text { TAP } & \text { Tip-associated protein } \\ \text { TPB } & \text { transport buffer } \\ \text { Triton X-100 } & \text { 4-octylphenol polyethoxylate } \\ \text { Tween 20 } & \text { polyoxyethylene (20) sorbitan monolaurate } \\ \text { UV } & \text { ultraviolet } \\ \text { V/v } & \text { volume per volume } \\ \text { W/o } & \text { without } \\ \text { W/v } & \text { weight per volume } \\ \text { WB } & \text { western blot } \\ \text { WGA } & \text { wheat germ agglutinin } \\ \text { WT } & \text { wild type } \\ \text { ZZ } & \text { protein tag (S. aureus protein A IgG-binding domain) }\end{array}$




\section{Acknowledgments}

I want to thank Prof. Ralph Kehlenbach for giving me the opportunity to work on this project, his continuous support and giving me the possibility to go on conferences.

The members of my thesis committee Prof. Heike Krebber and Prof. Jörg Großhans I thank for helpful discussions and inputs to my project.

Special thanks go to Christiane Spillner for taking care of general things in the lab, creating a positive working atmosphere and her experimental support during the last months of my PhD. Further, I am very grateful to Annegret Nath for doing the initial overexpression screen for potential importin 13 cargoes.

All current (Marina Blenski, Mohamed Hamed, Christina James, Floriane Lagadec, Marret Müller, Christiane Spillner) and former members (Cara Jamieson, Annegret Nath, Janine Pfaff, Sarah Port, Kalpana Rajanala, Janssel Reyes del Castillo) of the lab, I thank for the good working atmosphere and also the nice times outside of the lab. Special thanks to Dr. Janine Pfaff, Dr. Marret Müller, Christina James, Kirstin Runge and Anne Clancy for proof-reading parts of the thesis.

I thank the whole Department of Molecular Biology for the supportive and positive working environment.

A big thank you goes to my collaborators Dr. Oliver Valerius und Dr. Kerstin Schmitt for the quick analysis of my MS-samples, showing me how to prepare my samples for mass spectrometry, introducing me to the Proteome Discoverer, MaxQuant and Perseus software, doing the Proteome Discoverer analysis and answering all of my questions.

The Göttingen Graduate School for Neurosciences, Biophysics, and Molecular Biosciences (GGNB) I thank for the opportunity to participate in method courses, go to retreats and providing funding to go on a conference.

Finally, I would like to thank all the people, who supported and influenced me in some way or another during the years of my PhD. 


\section{Publications}

Halder K, Dölker N, Van Q, Gregor I, Dickmanns A, Baade I, Kehlenbach RH, Ficner R, Enderlein J, Grubmüller H, Neumann H. (2015) MD simulations and FRET reveal an environment-sensitive conformational plasticity of importin- $\beta$. Biophys J. 109, 277-86.

Parts of this thesis were published in Molecular \& Cellular Proteomics on 17th April 2018:

Baade I, Spillner C, Schmitt K, Valerius O, Kehlenbach RH. (2018) Extensive identification and in-depth validation of importin 13 cargoes. Mol Cell Proteomics. doi: 10.1074/mcp.RA118.000623. 
